\title{
Stereocontrolled Formation of Amino Acids and N-Heterocycles Bearing a Quaternary Chiral Carbon.
}

Stéphanie Roy and Claude Spino*

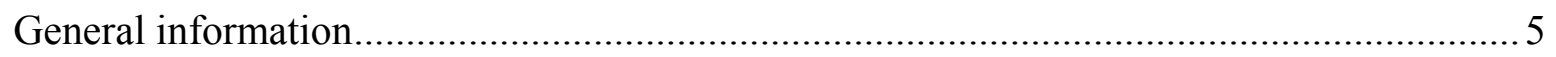

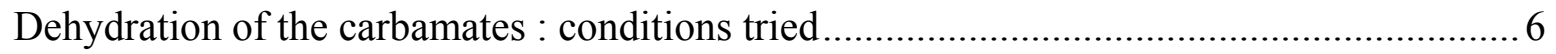

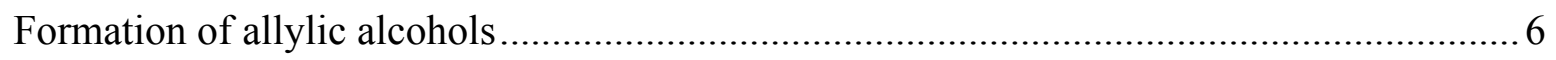

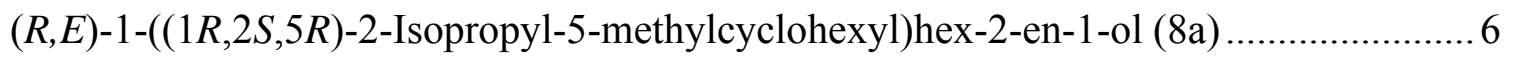

$(R, E)-1-((1 R, 2 S, 5 R)-2-$ Isopropyl-5-methylcyclohexyl)-4,4-dimethylpent-2-en-1-ol (8b). 6

$(S, E)-1-((1 R, 2 S, 5 R)-2-$ Isopropyl-5-methylcyclohexyl)-3-(trimethylsilyl)prop-2-en-1-ol .7

$(S, E)-1-((1 R, 2 S, 5 R)$-2-Isopropyl-5-methylcyclohexyl)-3-methylhex-2-en-1-ol (8d) ........8

$(S, E)-1-((1 R, 2 S, 5 R)$-2-Isopropyl-5-methylcyclohexyl)-3-phenylbut-2-en-1-ol (8e) $\ldots \ldots \ldots . . .8$

(S,E)-3-Benzyl-1-((1R,2S,5R)-2-isopropyl-5-methylcyclohexyl)but-2-en-1-ol (8f)......... 9

(S,E)-1-((1R,2S,5R)-2-Isopropyl-5-methylcyclohexyl)hex-2-en-1-ol (9a) ....................... 9

(S,E)-1-((1R,2S,5R)-2-Isopropyl-5-methylcyclohexyl)-4,4-dimethylpent-2-en-1-ol (9b) . 9

$(R, E)-1-((1 R, 2 S, 5 R)-2-$ Isopropyl-5-methylcyclohexyl)-3-(trimethylsilyl)prop-2-en-1-ol

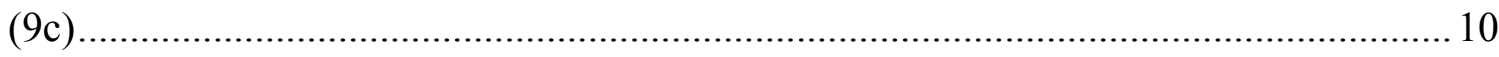

Minor allylic alcohols from major allylic alcohols via oxidation/reduction sequence.......... 11

$(R, E)-1-((1 R, 2 S, 5 R)-2-$ Isopropyl-5-methylcyclohexyl)-3-methylhex-2-en-1-ol (9d) ...... 11

$(R, E)-1-((1 R, 2 S, 5 R)-2-I s o p r o p y l-5-m e t h y l c y c l o h e x y l)-3$-phenylbut-2-en-1-ol (9e) ....... 12

$(R, E)$-3-Benzyl-1-((1R,2S,5R)-2-isopropyl-5-methylcyclohexyl)but-2-en-1-ol (9f) ....... 12

(E)-1-((1R,2S,5R)-2-Isopropyl-5-methylcyclohexyl)hex-2-en-1-one (20a) ..................... 13

(E)-1-((1R,2S,5R)-2-Isopropyl-5-methylcyclohexyl)-4,4-dimethylpent-2-en-1-one (20b)

(E)-1-((1R,2S,5R)-2-Isopropyl-5-methylcyclohexyl)-3-(trimethylsilyl)prop-2-en-1-one (20c)

(E)-1-((1R,2S,5R)-2-Isopropyl-5-methylcyclohexyl)-3-methylhex-2-en-1-one (20d) ..... 14

(E)-1-((1R,2S,5R)-2-Isopropyl-5-methylcyclohexyl)-3-phenylbut-2-en-1-one (20e) ...... 14 
(E)-3-Benzyl-1-((1R,2S,5R)-2-isopropyl-5-methylcyclohexyl)but-2-en-1-one (20f) ...... 14

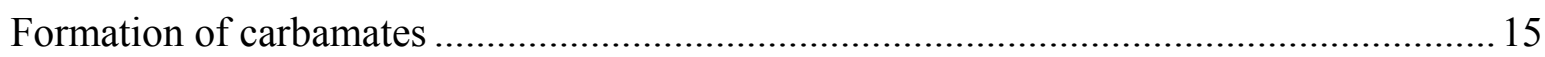

$(R, E)-1-((1 R, 2 S, 5 R)-2-$ Isopropyl-5-methylcyclohexyl)hex-2-enyl carbamate (11a) ...... 15

$(S, E)-1-((1 R, 2 S, 5 R)-2-I s o p r o p y l-5-m e t h y l c y c l o h e x y l) h e x-2-e n y l$ carbamate (21a)....... 15

$(R, E)-1-((1 R, 2 S, 5 R)-2-I s o p r o p y l-5-m e t h y l c y c l o h e x y l)-4,4-d i m e t h y l p e n t-2-e n y l$

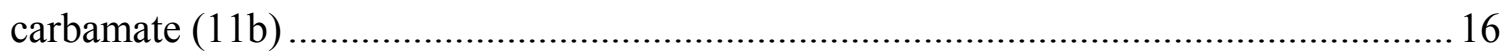

(S,E)-1-((1R,2S,5R)-2-Isopropyl-5-methylcyclohexyl)-4,4-dimethylpent-2-enyl

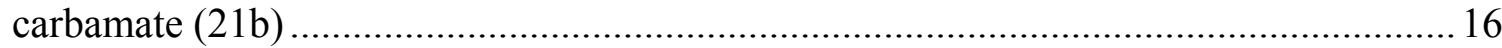

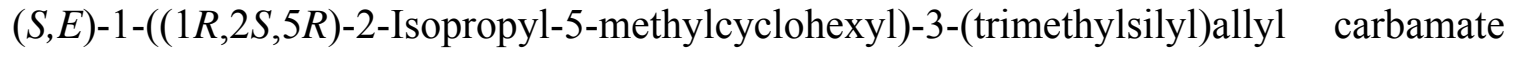

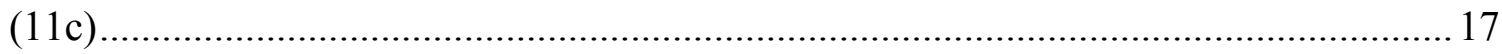

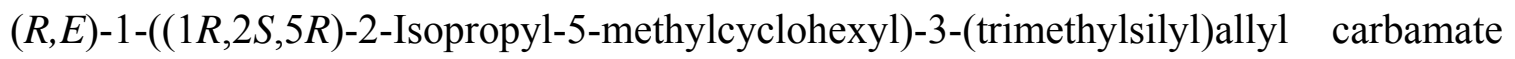
(21c) 17

$(S, E)-1-((1 R, 2 S, 5 R)-2-I s o p r o p y l-5-m e t h y l c y c l o h e x y l)-3-m e t h y l h e x-2-e n y l \quad$ carbamate 18

$(R, E)-1-((1 R, 2 S, 5 R)-2-I s o p r o p y l-5-m e t h y l c y c l o h e x y l)-3-m e t h y l h e x-2-e n y l \quad$ carbamate (21d) 18

$(S, E)-1-((1 R, 2 S, 5 R)-2-I s o p r o p y l-5-m e t h y l c y c l o h e x y l)-3-p h e n y l b u t-2-e n y l \quad$ carbamate

(R,E)-1-((1R,2S,5R)-2-Isopropyl-5-methylcyclohexyl)-3-phenylbut-2-enyl carbamate $(21 \mathrm{e})$ 19

(S,E)-1-((1R,2S,5R)-2-Isopropyl-5-methylcyclohexyl)-3-methyl-4-phenylbut-2-enyl carbamate (11f). 20

(R,E)-1-((1R,2S,5R)-2-Isopropyl-5-methylcyclohexyl)-3-methyl-4-phenylbut-2-enyl carbamate (21f) 20

Formation of allylisocyanates. 21

$(1 S, 2 S, 4 R)-2-((R, E)-3-$ Isocyanatohex-1-enyl)-1-isopropyl-4-methylcyclohexane (6a)...21 (1S,2S,4R)-2-((S,E)-3-Isocyanatohex-1-enyl)-1-isopropyl-4-methylcyclohexane (22a) . 21 (1S,2S,4R)-2-((S,E)-3-Isocyanato-4,4-dimethylpent-1-enyl)-1-isopropyl-4methylcyclohexane $(6 b)$ 22 (1S,2S,4R)-2-((R,E)-3-Isocyanato-4,4-dimethylpent-1-enyl)-1-isopropyl-4methylcyclohexane $(22 b)$. 
((R,E)-1-Isocyanato-3-((1S,2S,5R)-2-isopropyl-5-methylcyclohexyl)allyl)-trimethylsilane (6c)

((S,E)-1-Isocyanato-3-((1S,2S,5R)-2-isopropyl-5-methylcyclohexyl)allyl)-trimethylsilane (22c) 23

$(1 S, 2 S, 4 R)-2-((R, E)-3-I s o c y a n a t o-3-m e t h y l h e x-1-e n y l)-1-i s o p r o p y l-4-$ methylcyclohexane $(6 \mathrm{~d})$ 24

(1S,2S,4R)-2-((S,E)-3-Isocyanato-3-methylhex-1-enyl)-1-isopropyl-4-methylcyclohexane (22d)

1-((S,E)-2-Isocyanato-4-((1S,2S,5R)-2-isopropyl-5-methylcyclohexyl)but-3-en-2yl)benzene (6e) 25

1-((R,E)-2-Isocyanato-4-((1S,2S,5R)-2-isopropyl-5-methylcyclohexyl)but-3-en-2-

yl)benzene (22e) 25

1-((R,E)-2-Isocyanato-4-((1S,2S,5R)-2-isopropyl-5-methylcyclohexyl)-2-methylbut-3enyl)benzene (6f) 26

1-((S,E)-2-Isocyanato-4-((1S,2S,5R)-2-isopropyl-5-methylcyclohexyl)-2-methylbut-3-

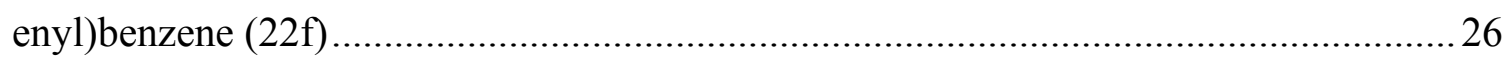

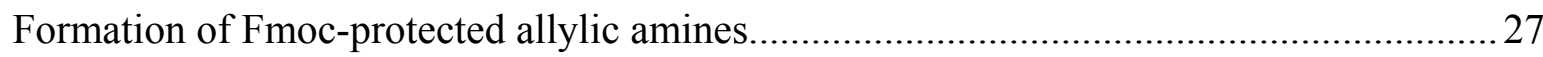

(9H-Fluoren-9-yl)methyl-(R,E)-1-((1S,2S,5R)-2-isopropyl-5-methylcyclohexyl)-3methylhex-1-en-3-ylcarbamate (14d) 27

(9H-Fluoren-9-yl)methyl-(S,E)-1-((1S,2S,5R)-2-isopropyl-5-methylcyclohexyl)-3-

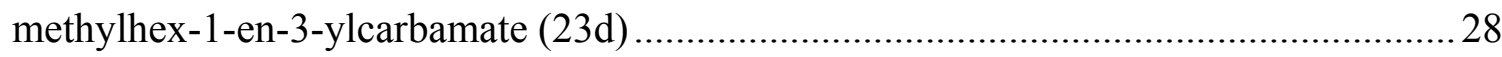
(9H-Fluoren-9-yl)methyl-(S,E)-4-((1S,2S,5R)-2-isopropyl-5-methylcyclohexyl)-2-

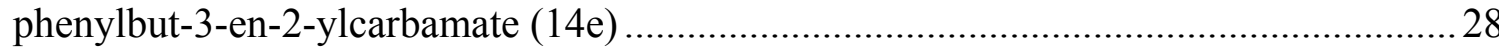
(9H-Fluoren-9-yl)methyl-( $R, E)-4-((1 S, 2 S, 5 R)-2$-isopropyl-5-methylcyclohexyl)-2phenylbut-3-en-2-ylcarbamate (23e). 29

(9H-Fluoren-9-yl)methyl-(R,E)-3-((1S,2S,5R)-2-isopropyl-5-methylcyclohexyl)-1(trimethylsilyl)allylcarbamate (14c) 29

(9H-Fluoren-9-yl)methyl-(S,E)-3-((1S,2S,5R)-2-isopropyl-5-methylcyclohexyl)-1(trimethylsilyl)allylcarbamate (23c) 30

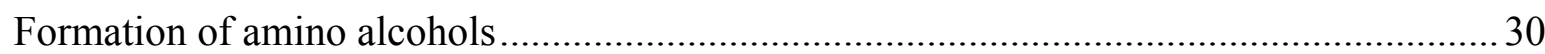

(9H-Fluoren-9-yl)methyl (R)-1-hydroxy-2-methylpentan-2-ylcarbamate (16d).............. 30

(9H-Fluoren-9-yl)methyl (R)-2-hydroxy-1-(trimethylsilyl)ethylcarbamate (16c) ............ 31 
(9H-Fluoren-9-yl)methyl (S)-2-hydroxy-1-(trimethylsilyl)ethylcarbamate (25c)............31

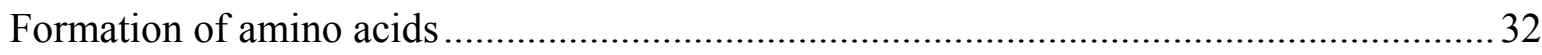

(R)-2-((9H-Fluoren-9-yl)methylcarbamate)-2-methylpentanoic acid (15d) ..................... 32

(S)-2-((9H-Fluoren-9-yl)methylcarbamate)-2-methylpentanoic acid (24d) ...................... 33

(R)-2-((9H-Fluoren-9-yl)methylcarbamate)-2-phenylpropanoic acid (15e) .................... 33

(S)-2-((9H-Fluoren-9-yl)methylcarbamate)-2-phenylpropanoic acid (24e) ...................... 34

(9H-Fluoren-9-yl)methyl (R)-2-formylpentan-2-ylcarbamate (26d).............................. 34

(9H-Fluoren-9-yl)methyl (S)-2-formylpentan-2-ylcarbamate (27d) ................................. 34

(9H-Fluoren-9-yl)methyl (R)-1-formyl-1-phenylethylcarbamate (26e) ............................35

(9H-Fluoren-9-yl)methyl (S)-1-formyl-1-phenylethylcarbamate (27e) ……………....... 35

$N$-((R,E)-1-((1S,2S,5R)-2-Isopropyl-5-methylcyclohexyl)-3-methylhex-1-en-3-

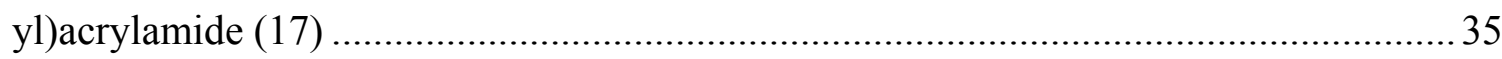

(R)-5-Methyl-5-propyl-1H-pyrrol-2(5H)-one (18) …………........................................ 36

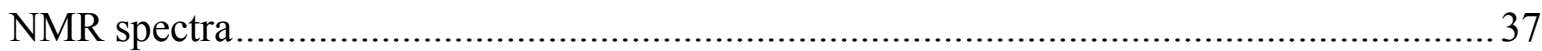




\title{
Stereocontrolled Formation of Amino Acids and N-Heterocycles Bearing a Quaternary Chiral Carbon.
}

\author{
Stéphanie Roy and Claude Spino*
}

\section{General information}

All reactions were performed under an inert atmosphere of argon in glassware that had been flame dried. Solvents were distilled from potassium/benzophenone ketyl $\left(\mathrm{Et}_{2} \mathrm{O}, \mathrm{THF}\right.$, benzene, toluene), from calcium hydride $\left(\mathrm{CH}_{2} \mathrm{Cl}_{2}, \mathrm{ClCH}_{2} \mathrm{CH}_{2} \mathrm{Cl}\right.$, triethylamine) or from potassium hydroxide ( $N, N, N, N$-tetramethylethylenediamine) prior to use. Reagents were purchased from Aldrich and used without purification. The synthesis of menthyl carboxaldehyde 4 was previously described. Flash chromatography was performed using Merck silica gel (230-400 Mesh ASTM) with solvents distilled prior to use. NMR spectra were recorded on a Bruker AC-300 spectrometer $\left({ }^{1} \mathrm{H}\right.$ NMR at $300 \mathrm{MHz},{ }^{13} \mathrm{C}$ NMR at 75.5 $\mathrm{MHz}$ ). The following abbreviations were used: s, singlet, $\mathrm{d}$, doublet, $\mathrm{t}$, triplet, q, quartet, qi, quintet, sext, sextet, sept, septet. Chemical shifts are reported in ppm with the solvent resonance as the internal standard $\left(\mathrm{CDCl}_{3}: 7.26 \mathrm{ppm}\left({ }^{1} \mathrm{H} \mathrm{NMR}\right), 77.0 \mathrm{ppm}\left({ }^{13} \mathrm{C} \mathrm{NMR}\right)\right.$; $\mathrm{CD}_{3} \mathrm{OD}: 3.30$ ( ${ }^{1} \mathrm{H}$ NMR), $49.0 \mathrm{ppm}\left({ }^{13} \mathrm{C} \mathrm{NMR}\right)$; benzene- $d_{6}$ : $7.15 \mathrm{ppm}\left({ }^{1} \mathrm{H}\right.$ NMR $), 128.0$ $\left.\left({ }^{13} \mathrm{C} N M R\right)\right)$. Infrared spectra were recorded on a Perkin Elmer 1600 FTIR spectrometer with a thin layer of the product on a $\mathrm{NaCl}$ disk. HPLC analyses were performed on a $\mathrm{HP}$ 1100 apparatus; GC analyses were performed on a Agilent 6890 series apparatus; GCMS analyses were performed on a HP 5890 serie II instrument (25 m length, 25 $\mu$ OD, capillary Stabiliwax-DB5 column) coupled with a mass spectrometer (HP 5971). High-resolution spectrometry was performed on a ZAB-1F micromass spectrometer. Optical rotations $[\alpha]_{\mathrm{D}}{ }^{20}$ were measured on a Perkin Elmer 343 polarimeter. Diastereomeric and enantiomeric ratios were determined as specified below for individual compounds. 
Dehydration of the carbamates : conditions tried

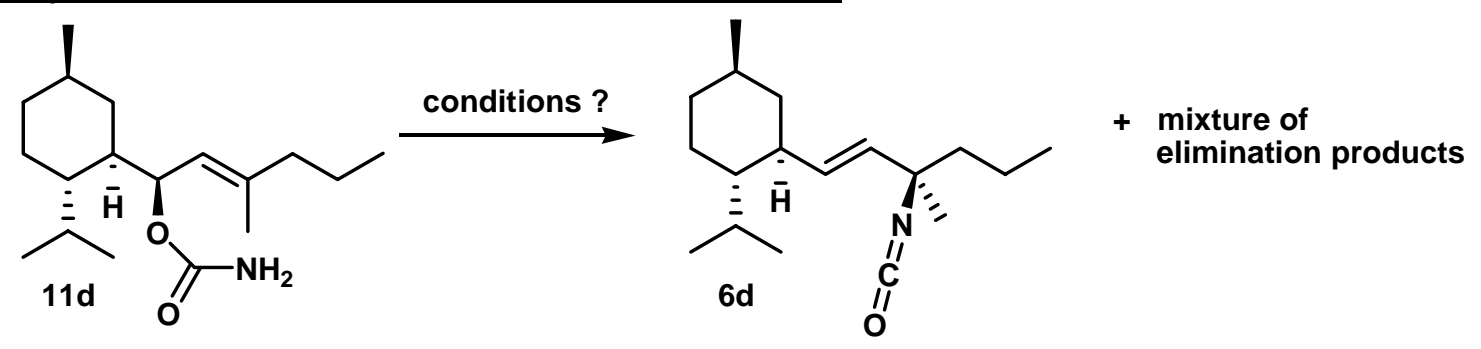

\begin{tabular}{|c|c|c|c|}
\hline Entry & conditions & $\%$ Yield of $6 d$ & $\%$ elimination \\
\hline 1 & $\mathrm{Tf}_{2} \mathrm{O}, \mathrm{iPr}_{2} \mathrm{EtN}, \mathrm{CH}_{2} \mathrm{Cl}_{2},-78^{\circ} \mathrm{C}$ & 37 & 45 \\
\hline 2 & $\mathrm{Tf}_{2} \mathrm{O}, \mathrm{CH}_{2} \mathrm{Cl}_{2},-78^{\circ} \mathrm{C}$ & 0 & $>98$ \\
\hline 3 & $\mathrm{Tf}_{2} \mathrm{O}, \mathrm{K}_{2} \mathrm{CO}_{3}, \mathrm{CH}_{2} \mathrm{Cl}_{2,}-78^{\circ} \mathrm{C}$ & 0 & $>98$ \\
\hline 4 & $\mathrm{Tf}_{2} \mathrm{O}, \mathrm{iPr}_{2} \mathrm{EtN}, \mathrm{CH}_{2} \mathrm{Cl}_{2}, 0^{\circ} \mathrm{C}$ & 0 & $>98$ \\
\hline 5 & $\mathrm{PPh}_{3}, \mathrm{CBr}_{4}, \mathrm{Et}_{3} \mathrm{~N}, \mathrm{CH}_{2} \mathrm{Cl}_{2},-20^{\circ} \mathrm{C}$ & 65 & 5 \\
\hline 6 & $\mathrm{Ti}(\mathrm{OtBu})_{4}, \mathrm{PhMe}$, reflux & no rx & -- \\
\hline 7 & $\mathrm{DCC}, \mathrm{CH}_{2} \mathrm{Cl}_{2}$, reflux & no rx & -- \\
\hline 8 & $\mathrm{POCl}_{3}, \mathrm{CH}_{2} \mathrm{Cl}_{2}, \mathrm{Et}_{3} \mathrm{~N}, 0^{\circ} \mathrm{C}$ to reflux & 14 & 84 \\
\hline 9 & TFAA, $\mathrm{Et}_{3} \mathrm{~N}, \mathrm{CH}_{2} \mathrm{Cl}_{2}, 0^{\circ} \mathrm{C}$ & $>99$ & -- \\
\hline
\end{tabular}

\section{Formation of allylic alcohols}

$(R, E)-1-((1 R, 2 S, 5 R)-2-I s o p r o p y l-5-m e t h y l c y c l o h e x y l) h e x-2-e n-1-o l ~(8 a)$

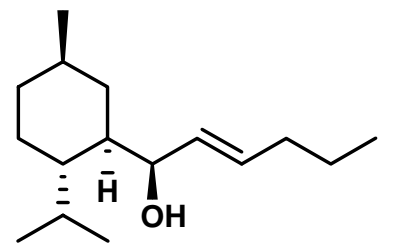

Prepared by the same procedure as per per 8b. Yield: . $>99 \%$ de (measured by gas chromatography). ${ }^{1} \mathbf{H}$ NMR $\left(\mathrm{CDCl}_{3}, 300 \mathrm{MHz}\right): \delta 5.64-5.49(\mathrm{~m}, 2 \mathrm{H}), 4.37(\mathrm{~d}, 1 \mathrm{H}, J=5.0$ $\mathrm{Hz}), 2.13(\mathrm{dq}, 1 \mathrm{H}, J=7.2,1.5 \mathrm{~Hz}), 2.03(\mathrm{q}, 2 \mathrm{H}, J=6.6 \mathrm{~Hz}), 1.70-1.63(\mathrm{~m}, 3 \mathrm{H}), 1.47-1.26$ $(\mathrm{m}, 3 \mathrm{H}), 1.01-0.78(\mathrm{~m}, 8 \mathrm{H}), 0.90(\mathrm{dd}, 6 \mathrm{H}, J=7.2,2.2 \mathrm{~Hz}), 0.76(\mathrm{~d}, 3 \mathrm{H}, J=6.6 \mathrm{~Hz}) . \mathbf{I R}$ (neat, $\mathrm{cm}^{-1}$ ): 3378 (br), 2954, 2923, 2871, 1640, 1455, 970. LRMS (m/z, (relative intensity)): $238\left(\mathrm{M}^{+}, 14\right), 139$ (14), 99 (60), $84(100)$. HRMS calcd for $\mathrm{C}_{16} \mathrm{H}_{30} \mathrm{O}: 238.2297$, found 238.2303. $[\alpha]_{\mathrm{D}}{ }^{20}=-29.3\left(c=2.87, \mathrm{CHCl}_{3}\right)$. 


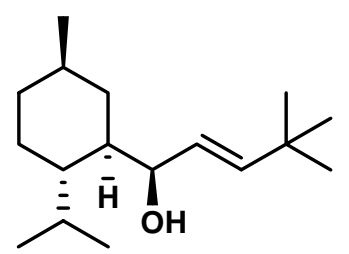

In a $250 \mathrm{~mL}$ round-bottomed flask was dissolved (E)-3,3-dimethyl-1-iodo-1-butene (3.00 g, $14.3 \mathrm{mmol})$ in $\mathrm{Et}_{2} \mathrm{O}(45 \mathrm{~mL})$. This solution was cooled to $-78^{\circ} \mathrm{C}$ and tert-butyllithium $(1.7$ $\mathrm{M}$ in pentane, $16.8 \mathrm{~mL}, 28.6 \mathrm{mmol})$ was added dropwise. The reaction mixture was stirred at $-78{ }^{\circ} \mathrm{C}$ for $30 \mathrm{~min}$ and $2 \mathrm{~h}$ at $23{ }^{\circ} \mathrm{C}$. Then, the mixture was cooled again to $-78{ }^{\circ} \mathrm{C}$ and a solution of $\mathrm{AlMe}_{3}(2.0 \mathrm{M}$ in hexanes, $21.4 \mathrm{~mL}, 42.8 \mathrm{mmol})$ was added. Then, a solution of p-menthane-3-carboxaldehyde 4 (2.00 g, $11.9 \mathrm{mmol})$ dissolved in $\mathrm{Et}_{2} \mathrm{O}(15 \mathrm{~mL})$ was added dropwise and the reaction mixture was stirred at $-78{ }^{\circ} \mathrm{C}$ for $3 \mathrm{~h}$. The reaction mixture was then allowed to warm to $0{ }^{\circ} \mathrm{C}$ and treated with a saturated solution of $\mathrm{NaHCO}_{3}$ and stirred in a $1 \mathrm{~N}$ aqueous solution of $\mathrm{HCl}$ until dissolution of the white solid. The aqueous phase was extracted with 3 portions of $\mathrm{Et}_{2} \mathrm{O}$ and the combined organic extracts were washed once with water and once with brine, dried over anhydrous $\mathrm{MgSO}_{4}$, filtered and concentrated under reduced pressure to give a yellow oil. The crude product was purified on silica gel with 5\% ethyl acetate in hexanes as eluant to afford a white solid $(2.81 \mathrm{~g}, 93 \%)$. $>99 \%$ de (measured by gas chromatography). m.p.: $49-50{ }^{\circ} \mathrm{C} .{ }^{1} \mathbf{H}$ NMR $\left(\mathrm{CDCl}_{3}, 300 \mathrm{MHz}\right): \delta 5.63(\mathrm{~d}, 1 \mathrm{H}, J=$ $15.9 \mathrm{~Hz}), 5.43(\mathrm{dd}, 1 \mathrm{H}, J=15.9,6.1 \mathrm{~Hz}), 4.37$ (d, 1H, $J=5.5 \mathrm{~Hz}), 2.14$ (dqi, 1H, $J=7.2$, $2.8 \mathrm{~Hz}), 1.72-1.69(\mathrm{~m}, 3 \mathrm{H}), 1.45$ (br s, 1H), 1.36-1.21 (m, 3H), 1.11-0.73 (m, 2H), $1.02(\mathrm{~s}$, $9 \mathrm{H}), 0.92(\mathrm{~d}, 3 \mathrm{H}, J=7.2 \mathrm{~Hz}), 0.88(\mathrm{~d}, 3 \mathrm{H}, J=6.6 \mathrm{~Hz}), 0.76(\mathrm{~d}, 3 \mathrm{H}, J=7.2 \mathrm{~Hz}) . \mathbf{I R}$ $\left(\mathrm{CHCl}_{3}, \mathrm{~cm}^{-1}\right): 3361,1386,1364,975$. LRMS (m/z, (relative intensity)): $252\left(\mathrm{M}^{+}, 10\right), 195$ (12), 139 (15), 113 (100), 83 (75). HRMS calcd for $\mathrm{C}_{17} \mathrm{H}_{32} \mathrm{O}$ : 252.2453, found: 252.2455. $[\alpha]_{\mathrm{D}}^{20}=-20.2\left(c=2.16, \mathrm{CHCl}_{3}\right)$.

\section{(S,E)-1-((1R,2S,5R)-2-Isopropyl-5-methylcyclohexyl)-3-(trimethylsilyl)prop-2-en-1-ol} (8c)

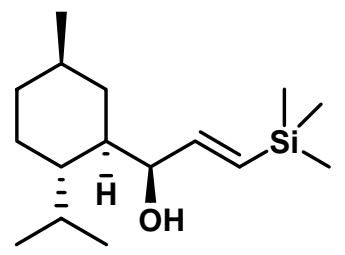

Prepared by the same procedure as per $\mathbf{8 b}$ except $\mathrm{AlMe}_{3}$ was omitted. The resulting oil was purified by flash chromatography (from hexanes to $5 \%$ diethyl ether in hexanes) to yield pure (S)-allylic alcohol 8c as the major isomer (930 mg, 40\%, white solid). $>99 \%$ de (measured by gas chromatography). m.p.: 47-50 ${ }^{\circ} \mathrm{C} \mathbf{1}_{\mathbf{H}} \mathbf{H M R}\left(300 \mathrm{MHz}, \mathrm{CDCl}_{3}\right) \delta(\mathrm{ppm})$ $6.06\left(\mathrm{dd}, 1 \mathrm{H}, J_{1}=19.0 \mathrm{~Hz}, J_{2}=3.6 \mathrm{~Hz}\right), 5.85\left(\mathrm{dd}, 1 \mathrm{H}, J_{1}=18.7 \mathrm{~Hz}, J_{2}=1.7 \mathrm{~Hz}\right), 4.42(\mathrm{dd}$, $\left.1 \mathrm{H}, J_{1}=3.9 \mathrm{~Hz}, J_{2}=1.7 \mathrm{~Hz}\right), 2.12\left(\mathrm{septd}, 1 \mathrm{H}, J_{1}=7.2 \mathrm{~Hz}, J_{2}=2.8 \mathrm{~Hz}\right), 1.74-1.65(\mathrm{~m}, 3 \mathrm{H})$, $1.56-1.51(\mathrm{~m}, 1 \mathrm{H}), 1.44-1.24(\mathrm{~m}, 4 \mathrm{H}), 1.07-0.71(\mathrm{~m}, 1 \mathrm{H}), 0.93(\mathrm{~d}, 3 \mathrm{H}, J=7.2 \mathrm{~Hz}), 0.87(\mathrm{~d}$, $3 \mathrm{H}, J=6.6 \mathrm{~Hz}), 0.77(\mathrm{~d}, 3 \mathrm{H}, J=7.2 \mathrm{~Hz}), 0.08(\mathrm{~s}, 9 \mathrm{H}) .{ }^{13} \mathrm{C} \mathbf{N M R}\left(75.5 \mathrm{MHz}, \mathrm{CDCl}_{3}\right) \delta$ (ppm) 148.6 (d), 127.8 (d), 72.5 (d), 44.1 (d), 43.0 (d), 35.1 (t), 33.6 (t), 32.7 (d), 26.3 (d), 24.3 (t), 22.8 (q), 21.5 (q), 15.4 (q), -1.2 (q). IR $\left(\mathrm{CHCl}_{3}, \mathrm{~cm}^{-1}\right)$ 3362(br), 2955, 2920, 2871, 1247, 866, 837. LRMS (m/z, relative intensity) $268\left(\mathrm{M}^{+}, 13\right), 139$ (40), 83 (87), 74 (100). HRMS calcd for $\mathrm{C}_{16} \mathrm{H}_{32} \mathrm{OSi}$ : 268.2222, found: 268.2229. $[\alpha]_{\mathrm{D}}{ }^{20}=-9.1\left(c=0.97, \mathrm{CHCl}_{3}\right)$. 
$(S, E)-1-((1 R, 2 S, 5 R)-2-I s o p r o p y l-5-m e t h y l c y c l o h e x y l)-3-m e t h y l h e x-2-e n-1-o l ~(8 d)$

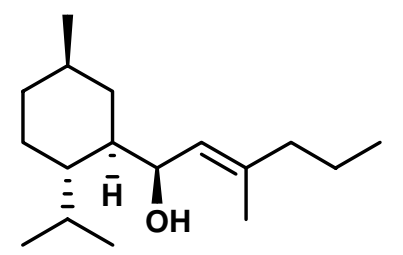

In a dry $100 \mathrm{~mL}$ round-bottomed flask, $\mathrm{Cp}_{2} \mathrm{ZrCl}_{2}(208 \mathrm{mg}, 0.71 \mathrm{mmol})$ was added to 6.2 $\mathrm{mL}$ of dichloromethane at $\mathrm{rt}$, followed by trimethylaluminium $(0.89 \mathrm{~mL}, 9.2 \mathrm{mmol})$ and the solution was stirred for $10 \mathrm{~min}$. The mixture was cooled to $0{ }^{\circ} \mathrm{C}$ and 1-pentyne $(0.3$ $\mathrm{mL}, 3.1 \mathrm{mmol}$ ) was added dropwise. The solution was stirred $16 \mathrm{~h}$ at $\mathrm{rt}$ and then cooled to $-78{ }^{\circ} \mathrm{C}$. Freshly purified $p$-menthane-3-carboxaldehyde 4 (399 $\left.\mathrm{mg}, 2.4 \mathrm{mmol}\right)$, dissolved in $7.9 \mathrm{~mL}$ of THF, was added dropwise. The reaction was stirred for $2 \mathrm{~h}$ and warmed slowly to $\mathrm{rt}$ during this period. Then, a saturated aqueous potassium carbonate solution was carefully added and the white precipitate obtained was dissolved with $1 \mathrm{~N} \mathrm{HCl}$. The aqueous phase was extracted with $3 \times 15 \mathrm{~mL}$ of dichloromethane. The organic layers were combined, washed with brine, dried with $\mathrm{MgSO}_{4}$, filtered and concentrated under reduced pressure. The resulting oil was purified by flash chromatography (from 5\% diethyl ether to $15 \%$ diethyl ether in hexanes) to yield pure (S)-allylic alcohol 8d as the major isomer (464 $\mathrm{mg}, 77 \%$, white solid) and pure (R)-allylic alcohol 9d as the minor isomer $(62 \mathrm{mg}, 10 \%$, colorless oil).

8d: $>99 \%$ de (measured by gas chromatography). m.p.: $30-31{ }^{\circ} \mathrm{C} .{ }^{1} \mathbf{H}$ NMR $(300 \mathrm{MHz}$, $\left.\mathrm{CDCl}_{3}\right) \delta(\mathrm{ppm}) 5.34(\mathrm{~d}, 1 \mathrm{H}, J=8.2 \mathrm{~Hz}), 4.67\left(\mathrm{dd}, 1 \mathrm{H}, J_{1}=8.3 \mathrm{~Hz}, J_{2}=3.9 \mathrm{~Hz}\right), 2.19$ (septd, $\left.1 \mathrm{H}, J_{1}=6.6 \mathrm{~Hz}, J_{2}=1.7 \mathrm{~Hz}\right), 1.98(\mathrm{t}, 2 \mathrm{H}, J=7.7 \mathrm{~Hz}), 1.73-1.65(\mathrm{~m}, 3 \mathrm{H}), 1.63(\mathrm{~s}$, $3 \mathrm{H}), 1.44$ (sext, $2 \mathrm{H}, J=7.5 \mathrm{~Hz}), 1.31-1.28(\mathrm{~m}, 3 \mathrm{H}), 1.12(\mathrm{~d}, 1 \mathrm{H}, J=4.4 \mathrm{~Hz}), 0.94(\mathrm{~d}, 3 \mathrm{H}, J$ $=7.1 \mathrm{~Hz}), 0.91-0.86(\mathrm{~m}, 9 \mathrm{H}), 0.79(\mathrm{~d}, 3 \mathrm{H}, J=6.6 \mathrm{~Hz}) .{ }^{13} \mathrm{C}$ NMR $\left(75.5 \mathrm{MHz}, \mathrm{CDCl}_{3}\right) \delta$ (ppm) 136.7 (s), 126.9 (d), 67.6 (d), 44.8 (d), 43.1 (t), 41.8 (d), 35.1 (t), 34.0 (t), 32.7 (d), $26.3(\mathrm{~d}), 24.2(\mathrm{t}), 22.8(\mathrm{q}), 21.6$ (q), $20.8(\mathrm{q}), 16.4(\mathrm{t}), 15.5$ (q), 13.7 (q). IR $\left(\mathrm{CHCl}_{3}, \mathrm{~cm}^{-1}\right)$ 3349(br), 2955, 2925, 2871, 1450. LRMS ( $\mathrm{m} / \mathrm{z}$, relative intensity) $252\left(\mathrm{M}^{+}, 22\right), 209((\mathrm{M}$ $\left.\left.\mathrm{C}_{3} \mathrm{H}_{7}\right)^{+}, 10\right), 113(100)$. HRMS calcd for $\mathrm{C}_{17} \mathrm{H}_{32} \mathrm{O}: 252.2453$, found: $252.2448 .[\alpha]_{D}{ }^{20}=-$ $51.1\left(c=1.15, \mathrm{CHCl}_{3}\right)$.

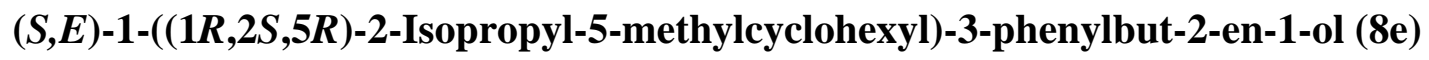<smiles>C/C(=C\C(O)[C@H]1CC(C)CC[C@@H]1C(C)C)c1ccccc1</smiles>

Prepared as per 8d. The resulting yellow oil was purified by flash chromatography (from $10 \%$ diethyl ether in hexanes to $15 \%$ diethyl ether in hexanes) to yield pure $(S)$-allylic alcohol $8 \mathrm{e}$ as the major isomer (1.33 g, 53\%, colorless oil). $>99 \%$ de (measured by gas chromatography). ${ }^{1} \mathbf{H}$ NMR $\left(300 \mathrm{MHz}, \mathrm{CDCl}_{3}\right) \delta(\mathrm{ppm}) 7.42(\mathrm{~d}, 2 \mathrm{H}, J=8.3 \mathrm{~Hz}), 7.34$ (t, $2 \mathrm{H}, J=7.4 \mathrm{~Hz}), 7.28-7.23(\mathrm{~m}, 1 \mathrm{H}), 5.95(\mathrm{~d}, 1 \mathrm{H}, J=7.7 \mathrm{~Hz}), 4.86\left(\mathrm{dd}, 1 \mathrm{H}, J_{1}=8.3 \mathrm{~Hz}, J_{2}=\right.$ $1.7 \mathrm{~Hz}), 2.25$ (septd, $\left.1 \mathrm{H}, J_{1}=6.6 \mathrm{~Hz}, J_{2}=2.8 \mathrm{~Hz}\right), 2.08(\mathrm{~s}, 3 \mathrm{H}), 1.78-1.69(\mathrm{~m}, 3 \mathrm{H}), 1.48-$ $1.26(\mathrm{~m}, 3 \mathrm{H}), 1.07-0.81(\mathrm{~m}, 3 \mathrm{H}), 0.98(\mathrm{~d}, 3 \mathrm{H}, J=7.2 \mathrm{~Hz}), 0.89$ (d, 3H, $J=6.6 \mathrm{~Hz}), 0.85$ 
$(\mathrm{d}, 3 \mathrm{H}, J=7.2 \mathrm{~Hz}) .{ }^{13} \mathrm{C}$ NMR $\left(75.5 \mathrm{MHz}, \mathrm{CDCl}_{3}\right) \delta(\mathrm{ppm}) 143.2(\mathrm{~s}), 135.1(\mathrm{~s}), 130.8(\mathrm{~d})$, 128.2 (d), 127.1 (d), 125.8 (d), 68.1 (d), 44.9 (d), 43.2 (d), 35.1 (t), 34.2 (t), 32.8 (d), 26.4 (d), 24.3 (t), 22.8 (q), 21.7 (q), 16.3 (q), 15.7 (q). IR (neat, $\mathrm{cm}^{-1}$ ) 3377(br), 2955, 2918, 2869, 1446, 757. LRMS ( $\mathrm{m} / \mathrm{z}$, relative intensity) $286\left(\mathrm{M}^{+}, 12\right), 147\left(\left(\mathrm{M}_{-} \mathrm{C}_{10} \mathrm{H}_{19}\right)^{+}, 100\right)$. HRMS calcd for $\mathrm{C}_{20} \mathrm{H}_{30} \mathrm{O}: 286.2297$, found: 286.2292. $[\alpha]_{\mathrm{D}}^{20}=-7.6\left(c=1.30, \mathrm{CHCl}_{3}\right)$.

\section{(S,E)-3-Benzyl-1-((1R,2S,5R)-2-isopropyl-5-methylcyclohexyl)but-2-en-1-ol (8f)}

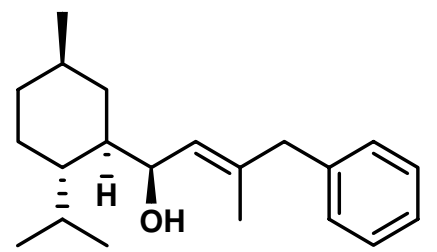

Prepared as per 8d. The resulting oil was purified by flash chromatography (from $5 \%$ to $15 \%$ diethyl ether in hexanes) to yield pure (S)-allylic alcohol $\mathbf{8 f}$ as the major isomer (2.916 $\mathrm{g}, 74 \%$, colorless oil) and pure $(R)$-allylic alcohol 9 f as the minor isomer $(175 \mathrm{mg}, 4 \%$, slightly yellow oil). 8f: $>99 \%$ de (measured by gas chromatography). ${ }^{1} \mathbf{H} \mathbf{N M R}\left(\mathrm{C}_{6} \mathrm{D}_{6}, 300\right.$ $\mathrm{MHz}): \delta 7.31-7.15(\mathrm{~m}, 5 \mathrm{H}), 5.58(\mathrm{~d}, 1 \mathrm{H}, J=7.8 \mathrm{~Hz}), 4.72-4.66(\mathrm{~m}, 1 \mathrm{H}), 3.35-3.22(\mathrm{~m}, 2 \mathrm{H})$, 2.38-2.27 (m, 1H), 1.94-1.88 (m, 1H), 1.82-1.72 (m, 2H), $1.60(\mathrm{~s}, 3 \mathrm{H}), 1.57-1.31(\mathrm{~m}, 3 \mathrm{H})$, $1.22(\mathrm{t}, 1 \mathrm{H}, J=11.8 \mathrm{~Hz}), 1.15-0.82(\mathrm{~m}, 9 \mathrm{H}), 0.89(\mathrm{~d}, 3 \mathrm{H}, J=6.8 \mathrm{~Hz}) ;{ }^{13} \mathbf{C} \mathbf{N M R}\left(\mathrm{C}_{6} \mathrm{D}_{6}, 75\right.$ $\mathrm{MHz}$ ) : $\delta 139.8$ (s), 134.7 (s), 130.3 (d), 128.9 (d), 128.4 (d), 126.2 (d), 67.4 (d), 46.3 (t), $45.2(\mathrm{~d}), 43.1$ (d), $35.3(\mathrm{t}), 34.4$ (t), 32.9 (d), 26.2 (d), 24.3 (t), 23.0 (q), 21.6 (q), 16.0 (q), 15.5 (q). IR (neat, $\left.\mathrm{cm}^{-1}\right): 3363,3026,2954,2870,1494,1102$; LRMS ( $\mathrm{m} / \mathrm{z}$ (relative

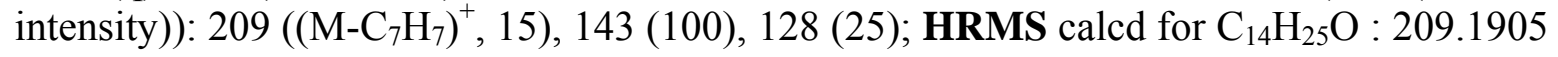
found : $209.1911 ;[\alpha]_{\mathrm{D}}-46.9^{\circ}\left(c=1.5, \mathrm{CHCl}_{3}\right)$.

\section{(S,E)-1-((1R,2S,5R)-2-Isopropyl-5-methylcyclohexyl)hex-2-en-1-ol (9a)}

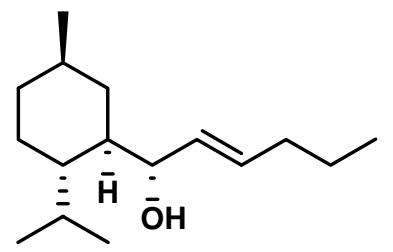

Prepared by the same procedure as per per $\mathbf{8 b}$ except $\mathrm{AlMe}_{3}$ was omitted. Yield:\%. A mixture of 8a and 9a was obtained and a sufficient quantity of 9a was isolated pure to carry out the sequence of reactions. 9a can also be prepared from an oxidation/reduction sequence from 8a. (See procedure for 9d). $>99 \%$ de (measured by gas chromatography). ${ }^{1} \mathbf{H}$ NMR $\left(\mathrm{CDCl}_{3}, 300 \mathrm{MHz}\right): \delta 5.68-5.60(\mathrm{~m}, 1 \mathrm{H}), 5.49(\mathrm{dd}, 1 \mathrm{H}, J=15.4,7.2 \mathrm{~Hz}), 4.37(\mathrm{dd}$, $1 \mathrm{H}, J=7.2,4.4 \mathrm{~Hz}), 2.04-1.89(\mathrm{~m}, 4 \mathrm{H}), 1.72-1.62(\mathrm{~m}, 3 \mathrm{H}), 1.44-1.34(\mathrm{~m}, 4 \mathrm{H}), 1.04-0.62$ $(\mathrm{m}, 6 \mathrm{H}), 0.90(\mathrm{dd}, 6 \mathrm{H}, J=2.2,7.2 \mathrm{~Hz}), 0.78(\mathrm{~d}, 3 \mathrm{H}, J=7.2 \mathrm{~Hz}) . \mathbf{I R}$ (neat, $\left.\mathrm{cm}^{-1}\right): 3358$, 1668, 970. LRMS (m/z, (relative intensity)): $238\left(\mathrm{M}^{+}, 4\right), 220\left(\left(\mathrm{M}-\mathrm{H}_{2} \mathrm{O}\right)^{+}, 1\right), 139(25), 123$ (8), 99 (86), 83 (100). HRMS calcd for $\mathrm{C}_{16} \mathrm{H}_{30} \mathrm{O}: 238.2297$, found: 238.2291 . $[\alpha]_{\mathrm{D}}{ }^{20}=-28.9$ $\left(c=2.21, \mathrm{CHCl}_{3}\right)$.

(S,E)-1-((1R,2S,5R)-2-Isopropyl-5-methylcyclohexyl)-4,4-dimethylpent-2-en-1-ol (9b) 


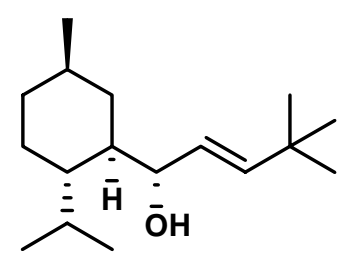

Prepared by the same procedure as per $\mathbf{8 b}$ except $\mathrm{AlMe}_{3}$ was omitted. Yield \%. A mixture of $\mathbf{8 b}$ and $9 \mathbf{b}$ was obtained and a sufficient quantity of $9 \mathbf{b}$ was isolated pure to carry out all the sequence. $\mathbf{9 b}$ can also be prepared from an oxidation/reduction sequence from $\mathbf{8 b}$. (See procedure for 9d). $>99 \%$ de (measured by gas chromatography). ${ }^{1} \mathbf{H}$ NMR $\left(\mathrm{CDCl}_{3}\right.$, $300 \mathrm{MHz}): \delta 5.68(\mathrm{~d}, 1 \mathrm{H}, J=15.9 \mathrm{~Hz}), 5.37(\mathrm{dd}, 1 \mathrm{H}, J=15.9,7.7 \mathrm{~Hz}), 4.35(\mathrm{dd}, 1 \mathrm{H}, J=$ 7.7, 3.9 Hz), 2.00-1.86 (m, 2H), 1.73-1.54 (m, 3H), $1.47(\mathrm{~m}, 1 \mathrm{H}), 1.33-1.22(\mathrm{~m}, 1 \mathrm{H}), 1.09-$ $0.60(\mathrm{~m}, 3 \mathrm{H}), 1.01(\mathrm{~s}, 9 \mathrm{H}), 0.89(\mathrm{~d}, 3 \mathrm{H}, J=6.6 \mathrm{~Hz}), 0.84(\mathrm{~d}, 3 \mathrm{H}, J=6.6 \mathrm{~Hz}), 0.77(\mathrm{~d}, 3 \mathrm{H}, J$ $=6.6 \mathrm{~Hz}) . \mathbf{I R}$ (neat, $\left.\mathrm{cm}^{-1}\right): 3361$ (br), 2958, 2917, 2869, 1386, 1364, 975. LRMS (m/z, (relative intensity)): $252\left(\mathrm{M}^{+}, 12\right), 195$ (14), 139 (22), 113 (84), 95 (55), 83 (100). HRMS calcd for $\mathrm{C}_{17} \mathrm{H}_{32} \mathrm{O}: 252.2453$, found: 252.2455 . $[\alpha]_{\mathrm{D}}{ }^{20}=-27.4\left(c=2.28, \mathrm{CHCl}_{3}\right)$.

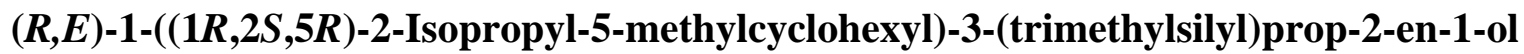
(9c)

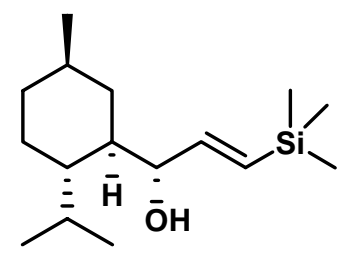

Prepared by the same procedure as per $\mathbf{8 b}$ except $\mathrm{AlMe}_{3}$ was omitted. Yield \%. A mixture of $\mathbf{8 c}$ and $9 \mathbf{c}$ was obtained and a sufficient quantity of $\mathbf{9 c}$ was isolated pure (305 mg, 13\%, slightly yellow oil) to carry out all the sequence. 9c can also be prepared from an oxidation/reduction sequence from 8c. (See procedure for 9d). 94\%de measured by gas chromatography. ${ }^{1} \mathbf{H}$ NMR $\left(300 \mathrm{MHz}, \mathrm{CDCl}_{3}\right) \delta(\mathrm{ppm}) 6.07\left(\mathrm{dd}, 1 \mathrm{H}, J_{1}=18.7 \mathrm{~Hz}, J_{2}=\right.$ $5.5 \mathrm{~Hz}), 5.87\left(\mathrm{~d}, 1 \mathrm{H}, J_{1}=19.8 \mathrm{~Hz}\right), 4.40(\mathrm{t}, 1 \mathrm{H}, J=4.7 \mathrm{~Hz}), 1.99\left(\mathrm{septd}, 1 \mathrm{H}, J_{1}=7.2 \mathrm{~Hz}, J_{2}\right.$ $=2.2 \mathrm{~Hz}), 1.82\left(\mathrm{dq}, 1 \mathrm{H}, J_{1}=12.7 \mathrm{~Hz}, J_{2}=3.3 \mathrm{~Hz}\right), 1.72-1.57(\mathrm{~m}, 3 \mathrm{H}), 1.35-1.26(\mathrm{~m}, 2 \mathrm{H})$, $1.04-0.85(\mathrm{~m}, 3 \mathrm{H}), 0.88(\mathrm{~d}, 3 \mathrm{H}, J=6.6 \mathrm{~Hz}), 0.87(\mathrm{~d}, 3 \mathrm{H}, J=7.2 \mathrm{~Hz}), 0.79(\mathrm{~d}, 3 \mathrm{H}, J=7.2$ $\mathrm{Hz}), 0.62(\mathrm{q}, 1 \mathrm{H}, J=12.1 \mathrm{~Hz}), 0.08(\mathrm{~s}, 9 \mathrm{H}) .{ }^{13} \mathrm{C}$ NMR $\left(75.5 \mathrm{MHz}, \mathrm{CDCl}_{3}\right) \delta(\mathrm{ppm}) 145.1$ (d), 130.4 (d), 73.8 (d), 44.7 (d), 44.0 (d), 35.1 (t), 34.3 (t), 32.5 (d), 26.2 (d), 24.2 (t), 22.7 (q), 21.4 (q), 15.2 (q), -1.3 (q). IR (neat, $\left.\mathrm{cm}^{-1}\right)$ 3589-3112, 2955, 2871, 1247. LRMS ( $\mathrm{m} / \mathrm{z}$, relative intensity) $268\left(\mathrm{M}^{+}, 7\right), 253\left(\left(\mathrm{M}_{-} \mathrm{CH}_{3}\right)^{+}, 5\right), 83(70), 74$ (100). HRMS calcd for $\mathrm{C}_{16} \mathrm{H}_{32} \mathrm{OSi}$ : 268.2222, found: $268.2229 .[\alpha]_{\mathrm{D}}^{20}=-37.5\left(c=1.27, \mathrm{CHCl}_{3}\right)$. 
Minor allylic alcohols from major allylic alcohols via oxidation/reduction sequence

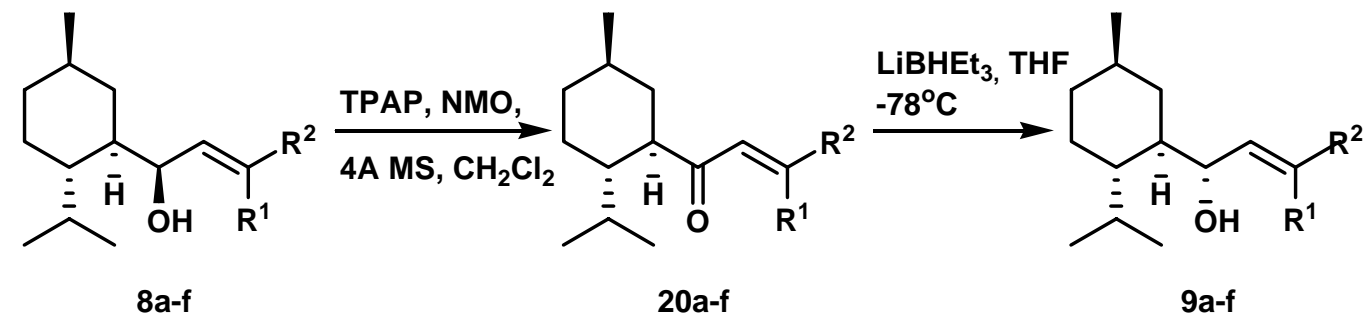

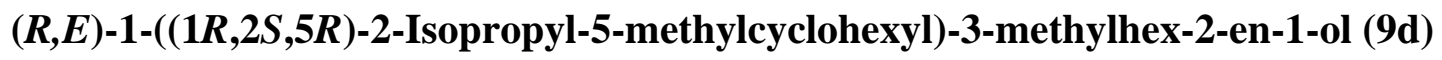

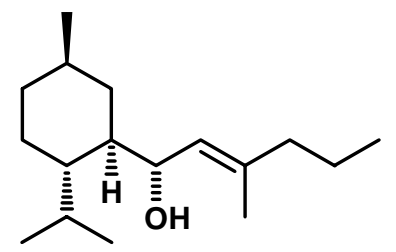

Prepared as per 8d. Alternatively, compound 9d can also be prepared from an oxidation/reduction sequence from 8d as follows: In a dry round-bottomed flask, the alcohol 8d (49 mg, $0.19 \mathrm{mmol})$ and $N$-methylmorpholine $N$-oxide $(34 \mathrm{mg}, 0.29 \mathrm{mg}$ ) was dissolved in dichloromethane $(0.4 \mathrm{~mL}, 0.5 \mathrm{M})$ containing $4 \AA$ molecular sieve $(97 \mathrm{mg}, 500$ $\mathrm{mg} / \mathrm{mmol})$. Finally, a catalytic amount of tetrapropylammonium perruthenate $(3 \mathrm{mg}, 0.001$ mmol) was added to the solution, which turned black within few min. The mixture was stirred at $\mathrm{rt}$ for $35 \mathrm{~min}$. The reaction mixture was then filtered through a pad of silica gel and eluted with dichloromethane. The crude enone 20d was placed in a dry round-bottomed flask and was dissolved in $3.4 \mathrm{~mL}$ of THF and the solution was cooled to $-78{ }^{\circ} \mathrm{C}$. A $1 \mathrm{M}$ solution of lithium triethylborohydride $(213 \mu \mathrm{L}, 0.213 \mathrm{mmol})$ was added dropwise. The reaction mixture was stirred at $-78{ }^{\circ} \mathrm{C}$ for $20 \mathrm{~min}$ after which $5 \mathrm{~mL}$ of a saturated aqueous solution of $\mathrm{NH}_{4} \mathrm{Cl}$ was added. The solution was allowed to warm to $25{ }^{\circ} \mathrm{C}$ and the solvent was evaporated under reduced pressure. Ammonium salts were dissolved with an additional $2 \mathrm{~mL}$ of water. The resulting solution was cooled to $0{ }^{\circ} \mathrm{C}$ and $0.5 \mathrm{~mL}$ of a $30 \mathrm{wt} \%$ solution of oxygen peroxide in water was added and the reaction mixture was stirred vigorously for 1h. It was then poured on a saturated aqueous solution of $\mathrm{NaHCO}_{3}$, and was extracted with $3 \times 5 \mathrm{~mL}$ of diethyl ether. The organic layers were combined, washed with brine, dried with $\mathrm{MgSO}_{4}$, filtered and concentrated under reduced pressure. The resulting oil was purified by flash chromatography ( $20 \%$ diethyl ether in hexanes) to yield $21 \mathrm{mg}(43 \%)$ of colorless oil. $>99 \%$ de (measured by gas chromatography). ${ }^{1} \mathbf{H}$ NMR $\left(300 \mathrm{MHz}, \mathrm{CDCl}_{3}\right) \delta(\mathrm{ppm}) 5.32$ $(\mathrm{d}, 1 \mathrm{H}, J=8.8 \mathrm{~Hz}), 4.63-4.57(\mathrm{~m}, 1 \mathrm{H}), 2.03-1.91(\mathrm{~m}, 3 \mathrm{H}), 1.80\left(\mathrm{septd}, 1 \mathrm{H}, J_{1}=7.2 \mathrm{~Hz}, J_{2}=\right.$ $2.8 \mathrm{~Hz}), 1.75-1.59(\mathrm{~m}, 2 \mathrm{H}), 1.70(\mathrm{~s}, 3 \mathrm{H}), 1.48-1.24(\mathrm{~m}, 3 \mathrm{H}), 1.15$ (d, $1 \mathrm{H}, J=3.9 \mathrm{~Hz}), 1.07-$ $0.71(\mathrm{~m}, 8 \mathrm{H}), 0.91(\mathrm{~d}, 3 \mathrm{H}, J=6.6 \mathrm{~Hz}), 0.82(\mathrm{~d}, 3 \mathrm{H}, J=6.6 \mathrm{~Hz}), 0.79(\mathrm{~d}, 3 \mathrm{H}, J=7.2 \mathrm{~Hz})$. ${ }^{13} \mathrm{C}$ NMR $\left(75.5 \mathrm{MHz}, \mathrm{CDCl}_{3}\right) \delta(\mathrm{ppm}) 136.6(\mathrm{~s}), 126.9$ (d), 67.5 (d), 44.8 (d), $43.1(\mathrm{t})$, $41.8(\mathrm{~d}), 35.1(\mathrm{t}), 34.0(\mathrm{t}), 32.7(\mathrm{~d}), 26.3(\mathrm{~d}), 24.2(\mathrm{t}), 22.8(\mathrm{q}), 21.6(\mathrm{q}), 20.8(\mathrm{q}), 16.4(\mathrm{t})$, 15.5 (q), 13.6 (q). IR (neat, $\mathrm{cm}^{-1}$ ) 3342(br), 2956, 2931, 2870, 1455, 1021, 999. LRMS $\left(\mathrm{m} / \mathrm{z}\right.$, relative intensity) $252\left(\mathrm{M}^{+}, 18\right), 209\left(\left(\mathrm{M}^{+}-\mathrm{C}_{3} \mathrm{H}_{7}\right), 10\right), 113(100)$. HRMS calcd for $\mathrm{C}_{17} \mathrm{H}_{32} \mathrm{O}: 252.2453$, found: $252.2448 .[\alpha]_{\mathrm{D}}^{20}=-23.4\left(c=1.07, \mathrm{CHCl}_{3}\right)$. 


\section{$(R, E)-1-((1 R, 2 S, 5 R)-2-I s o p r o p y l-5-m e t h y l c y c l o h e x y l)-3-p h e n y l b u t-2-e n-1-o l ~(9 e)$}

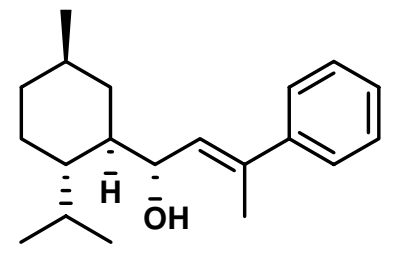

Prepared as per 9d from an oxidation/reduction sequence from 8e. The resulting yellow oil was purified by flash chromatography (from 10\% diethyl ether in hexanes to $15 \%$ diethyl ether in hexanes) to yield pure $(R)$-allylic alcohol 9e (185 mg, 57\%, white solid). $>99 \%$ de (measured by gas chromatography). m.p.: $69-71^{\circ} \mathrm{C} \mathbf{1}^{\mathbf{1}} \mathbf{H}$ NMR $\left(300 \mathrm{MHz}, \mathrm{CDCl}_{3}\right) \delta$ (ppm) 7.43-7.25 (m, 5H), $5.95\left(\mathrm{dd}, 1 \mathrm{H}, J_{1}=9.4 \mathrm{~Hz}, J_{2}=1.1 \mathrm{~Hz}\right), 4.78\left(\mathrm{dd}, 1 \mathrm{H}, J_{1}=9.4 \mathrm{~Hz}, J_{2}=\right.$ $3.3 \mathrm{~Hz}), 2.15(\mathrm{~d}, 3 \mathrm{H}, J=1.1 \mathrm{~Hz}), 2.02\left(\mathrm{dq}, 1 \mathrm{H}, J_{1}=12.4 \mathrm{~Hz}, J_{2}=3.3\right), 1.91$ (septd, $1 \mathrm{H}, J_{1}=$ $\left.6.6 \mathrm{~Hz}, J_{2}=2.2\right), 1.80-1.64(\mathrm{~m}, 3 \mathrm{H}), 1.43(\mathrm{br} \mathrm{s}, 1 \mathrm{H}), 1.41-1.26(\mathrm{~m}, 1 \mathrm{H}), 1.12-1.00(\mathrm{~m}, 2 \mathrm{H})$, 0.97-0.76 (m, 2H), $0.93(\mathrm{~d}, 3 \mathrm{H}, J=6.6 \mathrm{~Hz}), 0.86(\mathrm{~d}, 3 \mathrm{H}, J=7.2 \mathrm{~Hz}), 0.84(\mathrm{~d}, 3 \mathrm{H}, J=7.2$ Hz). ${ }^{13} \mathrm{C}$ NMR (75.5 MHz, $\left.\mathrm{CDCl}_{3}\right) \delta(\mathrm{ppm}) 143.4$ (s), 139.2 (s), 128.2 (d), 127.3 (d), 126.5 (d), 125.9 (d), 68.3 (d), 44.8 (d), 44.1 (d), $35.1(\mathrm{t}), 34.4$ (t), 32.6 (d), 26.7 (d), 24.1 (t), 22.8 (q), 21.6 (q), 16.4 (q), 15.3 (q). IR $\left(\mathrm{CHCl}_{3}\right) \vee\left(\mathrm{cm}^{-1}\right)$ 3245(br), 2952, 2916, 2866, 1449. LRMS $\left(\mathrm{m} / \mathrm{z}\right.$, relative intensity) $286\left(\mathrm{M}^{+}, 12\right), 147\left(\left(\mathrm{M}-\mathrm{C}_{10} \mathrm{H}_{19}\right)^{+}, 100\right)$. HRMS calcd for $\mathrm{C}_{20} \mathrm{H}_{30} \mathrm{O}: 286.2297$, found: 286.2289 . $[\alpha]_{\mathrm{D}}^{20}=-58.6\left(c=0.70, \mathrm{CHCl}_{3}\right)$.

\section{(R,E)-3-Benzyl-1-((1R,2S,5R)-2-isopropyl-5-methylcyclohexyl)but-2-en-1-ol (9f)}

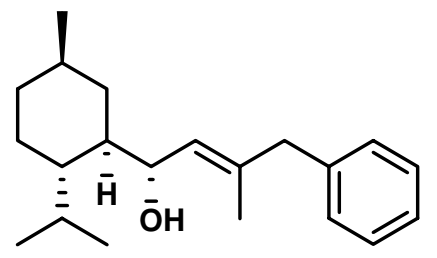

Prepared as per 9d from an oxidation/reduction sequence from $\mathbf{8 f}$. The resulting yellow oil was purified by flash chromatography (from 5\% diethyl ether in hexanes to $15 \%$ diethyl ether in hexanes) to yield pure (R)-allylic alcohol 9f. (125 mg, 45\%, 2 steps). $>99 \%$ de (measured by gas chromatography). ${ }^{1} \mathbf{H} \mathbf{R M N}\left(\mathrm{C}_{6} \mathrm{D}_{6}, 300 \mathrm{MHz}\right): \delta 7.18-7.05(\mathrm{~m}, 5 \mathrm{H}), 5.48$ (d, $1 \mathrm{H}, J=10.0 \mathrm{~Hz}), 4.60-4.52(\mathrm{~m}, 1 \mathrm{H}), 3.19$ (d, $1 \mathrm{H}, J=14.2 \mathrm{~Hz}), 3.12$ (d, $1 \mathrm{H}, J=14.2 \mathrm{~Hz}$ ), 2.20-2.13 (m, 1H), 1.95-1.84 (m, 1H), 1.76-1.60 (m, 3H), $1.50(\mathrm{~s}, 3 \mathrm{H}), 1.37-1.21(\mathrm{~m}, 1 \mathrm{H})$, 1.12-0.80 (m, 5H), 0.96 (d, 3H, J=6.7 Hz), 0.87 (d, $3 \mathrm{H}, \mathrm{J}=6.7 \mathrm{~Hz}), 0.80$ (d, 3H, J=7.3 Hz); ${ }^{13}$ C RMN $\left(\mathrm{C}_{6} \mathrm{D}_{6}, 75 \mathrm{MHz}\right): \delta 139.5$ (s), 138.1 (s), 128.5 (d), 128.0 (d), 126.0 (d), 125.9 (d), $67.3(\mathrm{~d}), 46.3(\mathrm{t}), 44.5(\mathrm{~d}), 44.3(\mathrm{~d}), 35.1(\mathrm{t}), 34.2(\mathrm{t}), 32.5(\mathrm{~d}), 26.2(\mathrm{~d}), 24.0(\mathrm{t}), 22.6$ (q), 21.2 (q), 15.7 (q), 15.0 (q); IR (neat, $\mathrm{cm}^{-1}$ ) :3363, 3026, 2954, 2870, 1494, 1102; SMBR $\left(\mathrm{m} / \mathrm{z}\right.$ (relative intensity)) : $209\left(\left(\mathrm{M}_{-} \mathrm{C}_{7} \mathrm{H}_{7}\right)^{+}, 15\right), 161$ (55), 143 (100); SMHR calcd for $\mathrm{C}_{14} \mathrm{H}_{25} \mathrm{O}: 209.1905$ found : $209.1911 ;[\alpha]_{\mathrm{D}}-9.4\left(c=0.67, \mathrm{CHCl}_{3}\right)$. 
(E)-1-((1R,2S,5R)-2-Isopropyl-5-methylcyclohexyl)hex-2-en-1-one (20a)

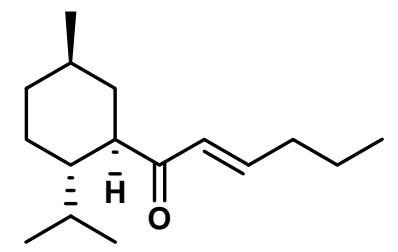

Colorless oil (72\% yield). ${ }^{1} \mathbf{H}$ NMR $\left(300 \mathrm{MHz}, \mathrm{CDCl}_{3}\right) \delta(\mathrm{ppm}) 6.88\left(\mathrm{dt}, 1 \mathrm{H}, J_{1}=15.4\right.$ $\left.\mathrm{Hz}, J_{2}=7.2 \mathrm{~Hz}\right), 6.16\left(\mathrm{dd}, 1 \mathrm{H}, J_{1}=17.0 \mathrm{~Hz}, J_{2}=1.7 \mathrm{~Hz}\right), 2.65\left(\mathrm{td}, 1 \mathrm{H}, J_{1}=11.3 \mathrm{~Hz}, J_{2}=\right.$ $3.3 \mathrm{~Hz}), 2.20\left(\mathrm{qd}, 2 \mathrm{H}, J_{1}=7.0 \mathrm{~Hz}, J_{2}=1.5 \mathrm{~Hz}\right), 1.76-1.67(\mathrm{~m}, 3 \mathrm{H}), 1.62-1.26(\mathrm{~m}, 4 \mathrm{H}), 1.50$ (q, $1 \mathrm{H}, J=7.3 \mathrm{~Hz}), 1.08-0.87(\mathrm{~m}, 6 \mathrm{H}), 0.89(\mathrm{~d}, 3 \mathrm{H}, J=6.6 \mathrm{~Hz}), 0.86(\mathrm{~d}, 3 \mathrm{H}, J=6.6 \mathrm{~Hz})$, 0.74 (d, 3H, $J=7.2 \mathrm{~Hz}$ ). IR (neat, $\left.\mathrm{cm}^{-1}\right)$ 2957, 2927, 2872, 1691, 1665, 1625. LRMS (m/z, relative intensity) $236\left(\mathrm{M}^{+}, 12\right), 193$ (100). HRMS calcd for $\mathrm{C}_{16} \mathrm{H}_{28} \mathrm{O}: 236.2140$, found: 236.2133 .

(E)-1-((1R,2S,5R)-2-Isopropyl-5-methylcyclohexyl)-4,4-dimethylpent-2-en-1-one (20b)<smiles>CC(C)[C@H]1CC[C@@H](C)CC1C(=O)/C=C/C(C)(C)C</smiles>

Colorless oil (68\% yield). ${ }^{1} \mathbf{H}$ NMR $\left(300 \mathrm{MHz}, \mathrm{CDCl}_{3}\right) \delta(\mathrm{ppm}) 6.87$ (d, 1H, J = $\left.16.0 \mathrm{~Hz}\right)$, $6.07(\mathrm{~d}, 1 \mathrm{H}, J=16.0 \mathrm{~Hz}), 2.67\left(\mathrm{td}, 1 \mathrm{H}, J_{1}=11.3 \mathrm{~Hz}, J_{2}=3.3 \mathrm{~Hz}\right), 1.76-1.53(\mathrm{~m}, 5 \mathrm{H}), 1.47-$ $1.30(\mathrm{~m}, 1 \mathrm{H}), 1.11-0.89(\mathrm{~m}, 3 \mathrm{H}), 1.09(\mathrm{~s}, 9 \mathrm{H}), 0.89$ (d, 3H, $J=6.6 \mathrm{~Hz}), 0.87$ (d, 3H, J = 7.2 Hz). $0.74(\mathrm{~d}, 3 \mathrm{H}, J=6.6 \mathrm{~Hz})$. IR (neat, $\mathrm{cm}^{-1}$ ) 2958, 2925, 2870, 1691, 1663, 1622. LRMS $\left(\mathrm{m} / \mathrm{z}\right.$, relative intensity) $250\left(\mathrm{M}^{+}, 12\right), 193$ (73), 111 (100). HRMS calcd for $\mathrm{C}_{17} \mathrm{H}_{30} \mathrm{O}$ : 250.2297, found: 250.2305 .

(E)-1-((1R,2S,5R)-2-Isopropyl-5-methylcyclohexyl)-3-(trimethylsilyl)prop-2-en-1-one (20c)<smiles>CC(C)[C@H]1CC[C@@H](C)CC1C(=O)/C=C/[Si](C)(C)C</smiles>

Colorless oil (67\% yield). ${ }^{1} \mathbf{H}$ NMR $\left(300 \mathrm{MHz}, \mathrm{CDCl}_{3}\right) \delta(\mathrm{ppm}) 7.11(\mathrm{~d}, 1 \mathrm{H}, J=18.7 \mathrm{~Hz})$, $6.53(\mathrm{~d}, 1 \mathrm{H}, J=19.3 \mathrm{~Hz}), 2.79\left(\mathrm{td}, 1 \mathrm{H}, J_{1}=11.0 \mathrm{~Hz}, J_{2}=3.3 \mathrm{~Hz}\right), 1.76-1.50(\mathrm{~m}, 5 \mathrm{H}), 1.46-$ $1.33(\mathrm{~m}, 1 \mathrm{H}), 1.08-0.90(\mathrm{~m}, 3 \mathrm{H}), 0.89(\mathrm{~d}, 3 \mathrm{H}, J=6.6 \mathrm{~Hz}), 0.86(\mathrm{~d}, 3 \mathrm{H}, J=6.6 \mathrm{~Hz}) .0 .73(\mathrm{~d}$, $3 \mathrm{H}, J=7.2 \mathrm{~Hz}), 0.15(\mathrm{~s}, 9 \mathrm{H})$. IR (neat, $\left.\mathrm{cm}^{-1}\right)$ 2957, 2922, 2871, 1690, 1667. LRMS (m/z,

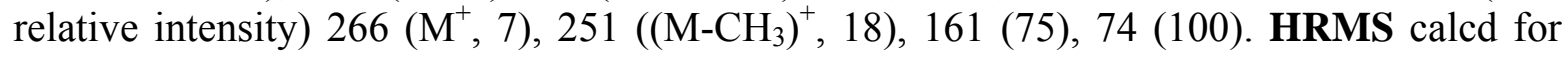
$\mathrm{C}_{16} \mathrm{H}_{30} \mathrm{OSi}$ : 266.2066, found: 266.2060 . 
(E)-1-((1R,2S,5R)-2-Isopropyl-5-methylcyclohexyl)-3-methylhex-2-en-1-one (20d)

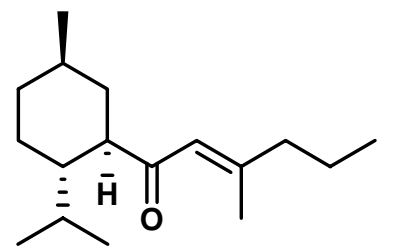

Colorless oil (39 mg, $81 \%$ yield). ${ }^{1} \mathbf{H}$ NMR $\left(300 \mathrm{MHz}, \mathrm{CDCl}_{3}\right) \delta(\mathrm{ppm}) 6.09(\mathrm{~s}, 1 \mathrm{H}), 2.38$ $\left(\mathrm{td}, 1 \mathrm{H}, J_{1}=11.6 \mathrm{~Hz}, J_{2}=3.3 \mathrm{~Hz}\right), 2.11(\mathrm{~s}, 3 \mathrm{H}), 2.10(\mathrm{t}, 2 \mathrm{H}, J=7.4 \mathrm{~Hz}) 1.74-1.29(\mathrm{~m}, 8 \mathrm{H})$, 1.06-0.91 (m, 6H), $0.88(\mathrm{~d}, 3 \mathrm{H}, J=7 \mathrm{~Hz}), 0,87(\mathrm{~d}, 3 \mathrm{H}, J=7 \mathrm{~Hz}), 0.75(\mathrm{~d}, 3 \mathrm{H}, J=6.6 \mathrm{~Hz})$. IR (neat, $\left.\mathrm{cm}^{-1}\right)$ 2966, 2878, 1681, 1621. LRMS ( $\mathrm{m} / \mathrm{z}$, relative intensity) $250\left(\mathrm{M}^{+}, 23\right), 207$

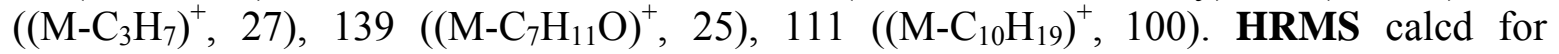
$\mathrm{C}_{17} \mathrm{H}_{30} \mathrm{O}: 250.2297$, found: 250.2302 .

(E)-1-((1R,2S,5R)-2-Isopropyl-5-methylcyclohexyl)-3-phenylbut-2-en-1-one (20e)<smiles>C/C(=C\C(=O)[C@@H]1CC(C)CC[C@H]1C(C)C)c1ccccc1</smiles>

Colorless oil (85\% yield). ${ }^{1} \mathbf{H}$ NMR $\left(300 \mathrm{MHz}, \mathrm{CDCl}_{3}\right) \delta(\mathrm{ppm})$ 7.51-7.46 (m, 2H), 7.43$7.36(\mathrm{~m}, 3 \mathrm{H}), 6.55(\mathrm{~d}, 1 \mathrm{H}, J=1.1 \mathrm{~Hz}), 2.54(\mathrm{~d}, 3 \mathrm{H}, J=1.1 \mathrm{~Hz}), 2.51\left(\mathrm{td}, 1 \mathrm{H}, J_{1}=11.8 \mathrm{~Hz}\right.$, $\left.J_{2}=3.0 \mathrm{~Hz}\right), 1.84-1.60(\mathrm{~m}, 4 \mathrm{H}), 1.45-1.26(\mathrm{~m}, 2 \mathrm{H}), 1.12-0.84(\mathrm{~m}, 3 \mathrm{H}), 0.90(\mathrm{~d}, 6 \mathrm{H}, J=6.6$ $\mathrm{Hz}$ ), 0.79 (d, 3H, $J=6.6 \mathrm{~Hz}$ ). IR (neat, $\mathrm{cm}^{-1}$ ) 3059, 3027, 2953, 2919, 2869, 1676, 1598. LRMS $\left(\mathrm{m} / \mathrm{z}\right.$, relative intensity) $284\left(\mathrm{M}^{+}, 11\right), 145$ (100), 115 (33), 91 (15). HRMS calcd for $\mathrm{C}_{20} \mathrm{H}_{28} \mathrm{O}: 284.2140$, found: 284.2146 .

(E)-3-Benzyl-1-((1R,2S,5R)-2-isopropyl-5-methylcyclohexyl)but-2-en-1-one (20f)<smiles>C/C(=C\C(=O)C1CC(C)CC[C@H]1C(C)C)Cc1ccccc1</smiles>

Colorless oil (302 mg, 87\% yield). ${ }^{\mathbf{1}} \mathbf{H}$ NMR $\left(300 \mathrm{MHz}, \mathrm{CDCl}_{3}\right) \delta(\mathrm{ppm})$ 7.34-7.24 (m, $3 \mathrm{H}), 7.17-7.15(\mathrm{~m}, 2 \mathrm{H}), 6.13(\mathrm{~s}, 1 \mathrm{H}), 3.43(\mathrm{~s}, 2 \mathrm{H}), 2.38\left(\mathrm{td}, 1 \mathrm{H}, J_{1}=12.1 \mathrm{~Hz}, J_{2}=3.3 \mathrm{~Hz}\right)$, $2.08(\mathrm{~s}, 3 \mathrm{H}), 1.75-1.51(\mathrm{~m}, 6 \mathrm{H}), 1.37-1.25(\mathrm{~m}, 1 \mathrm{H}), 1.06-0.94(\mathrm{~m}, 2 \mathrm{H}), 0.89(\mathrm{~d}, 3 \mathrm{H}, J=6.6$ $\mathrm{Hz}), 0.87(\mathrm{~d}, 3 \mathrm{H}, J=6.6 \mathrm{~Hz}), 0.74(\mathrm{~d}, 3 \mathrm{H}, J=7.2 \mathrm{~Hz}) .{ }^{13} \mathrm{C} \mathbf{N M R}\left(75.5 \mathrm{MHz}, \mathrm{CDCl}_{3}\right) \delta$ (ppm) 205.5 (s), 157.1 (s), 138.1 (s), 129.2 (d), 128.7 (d), 126.8 (d), 124.5 (d), 55.5 (d), $47.5(\mathrm{t}), 44.5(\mathrm{~d}), 38.9(\mathrm{t}), 34.8(\mathrm{t}), 32.6(\mathrm{~d}), 29.0(\mathrm{~d}), 24.2(\mathrm{t}), 22.5(\mathrm{q}), 21.6(\mathrm{q}), 19.3(\mathrm{q})$, 16.3 (q). IR (neat, $\mathrm{cm}^{-1}$ ) 2954, 2921, 2870, 1681, 1616. LRMS ( $\mathrm{m} / \mathrm{z}$, relative intensity) $298\left(\mathrm{M}^{+}, 48\right), 207$ (55), 159 (100). HRMS calcd for $\mathrm{C}_{21} \mathrm{H}_{30} \mathrm{O}: 298.2297$, found: 298.2303. 


\section{Formation of carbamates}

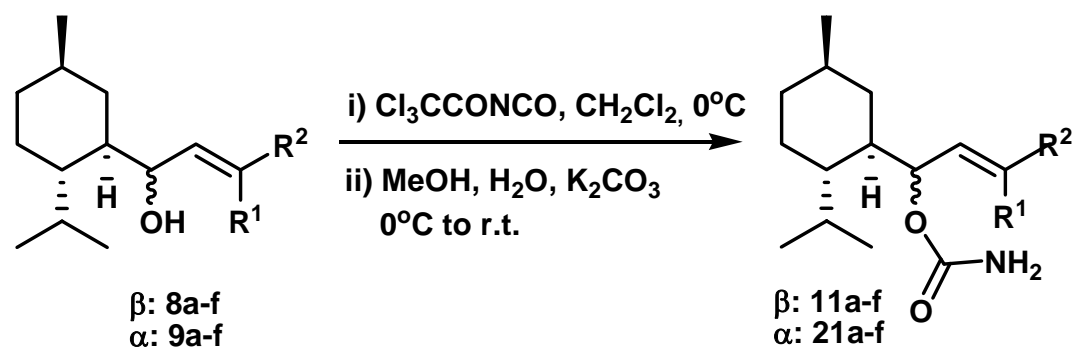

(R,E)-1-((1R,2S,5R)-2-Isopropyl-5-methylcyclohexyl)hex-2-enyl carbamate (11a)<smiles>CCC/C=C/[C@H](OC(N)=O)C1C[C@H](C)CC[C@H]1C(C)C</smiles>

Prepared as per 11f from allylic alcohol 8a. The resulting oil was purified by flash chromatography $(40 \%$ diethyl ether in hexanes) to yield pure $(R)$-allylcarbamate 11a (285 mg, 96\%) as a white solid. m.p.: $101-102{ }^{\circ} \mathrm{C} \mathbf{1}_{\mathbf{H}} \mathbf{N M R}\left(300 \mathrm{MHz}, \mathrm{CDCl}_{3}\right) \delta$ (ppm) 5.61 $\left(\mathrm{dt}, 1 \mathrm{H}, J_{1}=15.4 \mathrm{~Hz}, J_{2}=7.3 \mathrm{~Hz}\right), 5.43\left(\mathrm{dd}, 1 \mathrm{H}, J_{1}=15.4 \mathrm{~Hz}, J_{2}=5.5 \mathrm{~Hz}\right), 5.31(\mathrm{br} \mathrm{d}, 1 \mathrm{H}$, $J=6.1 \mathrm{~Hz}), 4.54\left(\right.$ br s, 2H), $2.10\left(\operatorname{septd}, 1 \mathrm{H}, J_{1}=6.6 \mathrm{~Hz}, J_{2}=3.0 \mathrm{~Hz}\right), 2.0(\mathrm{q}, 2 \mathrm{H}, J=7.2$ $\mathrm{Hz}), 1.76-1.62(\mathrm{~m}, 3 \mathrm{H}), 1.49-1.10(\mathrm{~m}, 2 \mathrm{H}), 1.40(\mathrm{q}, 1 \mathrm{H}, J=7.5 \mathrm{~Hz}), 1.04-0.76(\mathrm{~m}, 8 \mathrm{H})$, $0.90(\mathrm{~d}, 3 \mathrm{H}, J=7.2 \mathrm{~Hz}), 0.88(\mathrm{~d}, 3 \mathrm{H}, J=6.6 \mathrm{~Hz}), 0.74(\mathrm{~d}, 3 \mathrm{H}, J=6.6 \mathrm{~Hz}) .{ }^{13} \mathbf{C} \mathbf{N M R}$ $\left(75.5 \mathrm{MHz} \mathrm{CDCl}_{3}\right) \delta(\mathrm{ppm}) 157.1$ (s), 132.3 (d), 127.6 (d), 75.1 (d), 43.6 (d), 43.0 (d), 35.3 $(\mathrm{t}), 35.0(\mathrm{t}), 34.4(\mathrm{t}), 32.7(\mathrm{~d}), 26.2(\mathrm{~d}), 24.1(\mathrm{t}), 22.7(\mathrm{q}), 22.2(\mathrm{q}), 21.5(\mathrm{t}), 15.3(\mathrm{q}), 13.6$ (q). IR $\left(\mathrm{CHCl}_{3}, \mathrm{~cm}^{-1}\right) 3476,3345,3291,3185,2958,2923,2872,1722,1693,1612,1388$, 1333. LRMS (m/z, relative intensity) $281\left(\mathrm{M}^{+}, 3\right), 238\left(\left(\mathrm{M}-\mathrm{C}_{3} \mathrm{H}_{7}\right)^{+}, 50\right), 220((\mathrm{M}-$ $\left.\left.\mathrm{HOCONH}_{2}\right)^{+}, 60\right), 177$ (95), 138, (60), 99 (95), 83 (100). HRMS calcd for $\mathrm{C}_{17} \mathrm{H}_{31} \mathrm{NO}_{2}$ : 281.2355, found: $281.2361 .[\alpha]_{\mathrm{D}}{ }^{20}=-14.2\left(c=1.00, \mathrm{CHCl}_{3}\right)$.

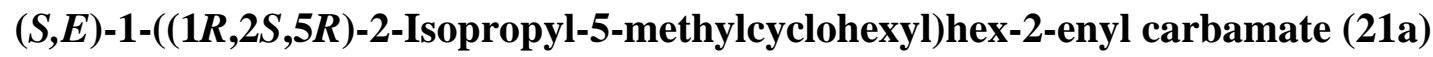<smiles>CCC/C=C/C(OC(N)=O)C1=CC(C)CC[C@H]1C(C)C</smiles>

Prepared as per 11f from allylic alcohol 9a. The resulting oil was purified by flash chromatography $(40 \%$ diethyl ether in hexanes) to yield pure $(S)$-allylcarbamate 21 a $(57$ mg, 97\%) as a white solid. m.p.: $73{ }^{\circ} \mathrm{C} \quad \mathbf{1}_{\mathbf{H}} \mathbf{N M R}\left(300 \mathrm{MHz}, \mathrm{CDCl}_{3}\right) \delta(\mathrm{ppm}) 5.72$ (dt, $\left.1 \mathrm{H}, J_{1}=14.3 \mathrm{~Hz}, J_{2}=7.0 \mathrm{~Hz}\right), 5.43\left(\mathrm{dd}, 1 \mathrm{H}, J_{1}=14.3 \mathrm{~Hz}, J_{2}=7.7 \mathrm{~Hz}\right), 5.36\left(\mathrm{dd}, 1 \mathrm{H}, J_{1}=\right.$ $\left.7.7 \mathrm{~Hz}, J_{2}=3.9 \mathrm{~Hz}\right), 4.63(\mathrm{br} \mathrm{s}, 2 \mathrm{H}), 2.09-1.94(\mathrm{~m}, 3 \mathrm{H}), 1.80\left(\mathrm{dq}, 1 \mathrm{H}, J_{1}=12.6 \mathrm{~Hz}, J_{2}=2.9\right.$ $\mathrm{Hz}), 1.71-1.61(\mathrm{~m}, 4 \mathrm{H}), 1.45-1.22(\mathrm{~m}, 3 \mathrm{H}), 1.34-1.22(\mathrm{~m}, 2 \mathrm{H}), 1.04-0.96(\mathrm{~m}, 1 \mathrm{H}), 0.90(\mathrm{~d}$, $3 \mathrm{H}, J=7.2 \mathrm{~Hz}), 0.88(\mathrm{~d}, 3 \mathrm{H}, J=6.6 \mathrm{~Hz}), 0.87(\mathrm{~d}, 3 \mathrm{H}, J=7.7 \mathrm{~Hz}), 0.84(\mathrm{t}, 3 \mathrm{H}, J=6.6)$. IR 
$\left(\mathrm{CHCl}_{3}, \mathrm{~cm}^{-1}\right)$ 3507, 3341, 3268, 2955, 2925, 2869, 1714, 1391, 1352, 1033. LRMS (m/z, relative intensity) $281\left(\mathrm{M}^{+}, 2\right), 238\left(\left(\mathrm{M}-\mathrm{C}_{3} \mathrm{H}_{7}\right)^{+}, 43\right), 220\left(\left(\mathrm{M}-\mathrm{HOCONH}_{2}\right)^{+}, 37\right), 177$ (77), 99 (71), 83 (100). HRMS calcd for $\mathrm{C}_{17} \mathrm{H}_{31} \mathrm{NO}_{2}$ : 281.2355, found: 281.2361. [ $\left.\alpha\right]_{\mathrm{D}}{ }^{20}=$ $36.2\left(c=1.09, \mathrm{CHCl}_{3}\right)$.

\section{(R,E)-1-((1R,2S,5R)-2-Isopropyl-5-methylcyclohexyl)-4,4-dimethylpent-2-enyl carbamate (11b)}

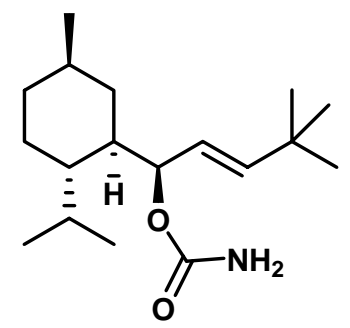

Prepared as per $\mathbf{1 1 f}$ from allylic alcohol $\mathbf{8 b}$. The resulting light yellow solid was purified by flash chromatography ( $15 \%$ ethyl acetate in hexanes) to yield pure $(R)$-allylcarbamate $\mathbf{1 1 b}$ $(240 \mathrm{mg}, 99 \%)$ as a white powder. m.p.: $97-98{ }^{\circ} \mathrm{C} \mathbf{1}_{\mathbf{H}} \mathbf{N M R}\left(300 \mathrm{MHz}, \mathrm{CDCl}_{3}\right) \delta(\mathrm{ppm})$ $5.63\left(\mathrm{dd}, 1 \mathrm{H}, J_{1}=17.9 \mathrm{~Hz}, J_{2}=3.5 \mathrm{~Hz}\right), 5.36-5.31(\mathrm{~m}, 2 \mathrm{H}), 4.53(\mathrm{br} \mathrm{s}, 2 \mathrm{H}), 2.12$ (septd, $\left.1 \mathrm{H}, J_{1}=6.6 \mathrm{~Hz}, J_{2}=2.8 \mathrm{~Hz}\right), 1.73-1.62(\mathrm{~m}, 3 \mathrm{H}), 1.49-1.41(\mathrm{~m}, 1 \mathrm{H}), 1.31-1.22(\mathrm{~m}, 1 \mathrm{H})$, 1.17-1.02 (m, 1H), $1.02(\mathrm{~s}, 9 \mathrm{H}), 1.01-0.70(\mathrm{~m}, 3 \mathrm{H}), 0.88(\mathrm{~d}, 6 \mathrm{H}, J=6.6 \mathrm{~Hz}), 0.75(\mathrm{~d}, 3 \mathrm{H}, J$ $=7.2 \mathrm{~Hz}) .{ }^{13} \mathrm{C}$ NMR $\left(75.5 \mathrm{MHz}, \mathrm{CDCl}_{3}\right) \delta(\mathrm{ppm}) 156.8(\mathrm{~s}), 143.1$ (d), 122.1 (d), $75.3(\mathrm{~d})$, 43.7 (d), 43.2 (d), 35.5 (t), 35.0 (t), 32.9 (s), 32.7 (d), 29.5 (q), 26.2 (d), 24.1 (t), 22.7 (q), 21.5 (q), 15.3 (q). IR $\left(\mathrm{CHCl}_{3}, \mathrm{~cm}^{-1}\right)$ 3510, 3342, 3262, 2962, 2874, 1712, 1390, 1337, 1032, 759. LRMS $\left(\mathrm{m} / \mathrm{z}\right.$, relative intensity) $295\left(\mathrm{M}^{+}, 1\right), 252\left(\left(\mathrm{M}-\mathrm{C}_{3} \mathrm{H}_{7}\right)^{+}, 27\right), 195(60)$, 177 (94), 113 (94), 95 (100), 83 (99). HRMS calcd for $\mathrm{C}_{18} \mathrm{H}_{33} \mathrm{NO}_{2}$ : 295.2511, found: 295.2504. $[\alpha]_{\mathrm{D}}^{20}=-17.2\left(c=0.95, \mathrm{CHCl}_{3}\right)$.

(S,E)-1-((1R,2S,5R)-2-Isopropyl-5-methylcyclohexyl)-4,4-dimethylpent-2-enyl carbamate (21b)

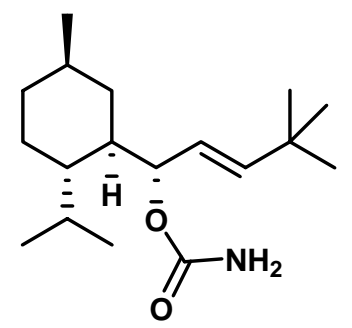

Prepared as per $11 \mathrm{f}$ from allylic alcohol $\mathbf{9 b}$. The resulting light yellow solid was purified by flash chromatography ( $45 \%$ diethyl ether in hexanes) to yield pure (S)-allylcarbamate $21 \mathbf{b}$ (86 mg, 98\%) as a white powder. m.p.: $105-106^{\circ} \mathrm{C} \mathbf{1}_{\mathbf{H}} \mathbf{N M R}\left(300 \mathrm{MHz}, \mathrm{CDCl}_{3}\right) \delta(\mathrm{ppm})$ $5.75(\mathrm{~d}, 1 \mathrm{H}, J=14.3 \mathrm{~Hz}), 5.37\left(\mathrm{dd}, 1 \mathrm{H}, J_{1}=7.4 \mathrm{~Hz}, J_{2}=3.6 \mathrm{~Hz}\right), 5.32\left(\mathrm{dd}, 1 \mathrm{H}, J_{1}=14.3\right.$ $\left.\mathrm{Hz}, J_{2}=8.3 \mathrm{~Hz}\right), 4.51(\mathrm{br} \mathrm{s}, 2 \mathrm{H}), 2.01\left(\mathrm{septd}, 1 \mathrm{H}, J_{1}=7.2 \mathrm{~Hz}, J_{2}=2.8 \mathrm{~Hz}\right), 1.81\left(\mathrm{dq}, 1 \mathrm{H}, J_{1}\right.$ $\left.=12.4 \mathrm{~Hz}, J_{2}=3.0 \mathrm{~Hz}\right), 1.74-1.55(\mathrm{~m}, 4 \mathrm{H}), 1.37-1.22(\mathrm{~m}, 1 \mathrm{H}), 1.08-0.67(\mathrm{~m}, 3 \mathrm{H}), 1.02(\mathrm{~s}$, 9H), $0.89(\mathrm{~d}, 3 \mathrm{H}, J=6.6 \mathrm{~Hz}), 0.84(\mathrm{~d}, 3 \mathrm{H}, J=7.2 \mathrm{~Hz}), 0.83(\mathrm{~d}, 3 \mathrm{H}, J=6.6 \mathrm{~Hz}) . \quad$ IR $\left(\mathrm{CHCl}_{3}, \mathrm{~cm}^{-1}\right) 3514,3342,2958,2865,1717,1386,1359,1350,1319,1037,759$. LRMS $\left(\mathrm{m} / \mathrm{z}\right.$, relative intensity) $295\left(\mathrm{M}^{+}, 1\right), 252\left(\left(\mathrm{M}-\mathrm{C}_{3} \mathrm{H}_{7}\right)^{+}, 30\right), 193(76), 177(90), 139(87)$ 
113 (100), 95 (96), 83 (97). HRMS calcd for $\mathrm{C}_{18} \mathrm{H}_{33} \mathrm{NO}_{2}$ : 295.2511, found: 295.2516. $[\alpha]_{\mathrm{D}}^{20}=-38.9\left(c=1.22, \mathrm{CHCl}_{3}\right)$.

(S,E)-1-((1R,2S,5R)-2-Isopropyl-5-methylcyclohexyl)-3-(trimethylsilyl)allyl carbamate (11c)

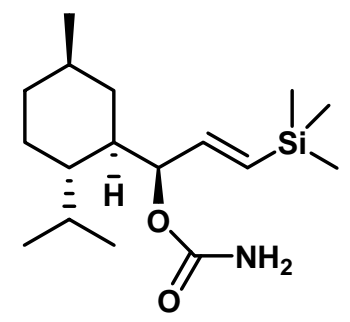

Prepared as per 11f from allylic alcohol 8c. The resulting light yellow solid was purified by flash chromatography ( $40 \%$ diethyl ether in hexanes) to yield pure $(S)$-allylcarbamate 11c (987 mg, 97\%) as a white solid. m.p.: $84-85{ }^{\circ} \mathrm{C} \mathbf{1}^{\mathbf{1}} \mathbf{H}$ NMR $\left(300 \mathrm{MHz}, \mathrm{CDCl}_{3}\right) \delta(\mathrm{ppm})$ $5.92\left(\mathrm{dd}, 1 \mathrm{H}, J_{1}=18.7 \mathrm{~Hz}, J_{2}=3.9 \mathrm{~Hz}\right), 5.76\left(\mathrm{dd}, 1 \mathrm{H}, J_{1}=18.7 \mathrm{~Hz}, J_{2}=1.7 \mathrm{~Hz}\right), 5.36-5.35$ (m, 1H), 4.92 (br s, 2H), 2.07 (septd, $\left.1 \mathrm{H}, J_{1}=6.6 \mathrm{~Hz}, J_{2}=2.8 \mathrm{~Hz}\right), 1.71-1.61(\mathrm{~m}, 3 \mathrm{H})$, 1.54-1.45 (m, 1H), 1.30-1.23 (m, 1H), 1.18-1.13 (m, 1H), 1.10-0.77 (m, 3H), $0.86(\mathrm{~d}, 3 \mathrm{H}, J$ $=7.2 \mathrm{~Hz}), 0.86(\mathrm{~d}, 3 \mathrm{H}, J=6.1 \mathrm{~Hz}), 0.74(\mathrm{~d}, 3 \mathrm{H}, J=6.6 \mathrm{~Hz}), 0.06(\mathrm{~s}, 9 \mathrm{H}) . \quad{ }^{13} \mathrm{C}$ NMR $\left(75.5 \mathrm{MHz}, \mathrm{CDCl}_{3}\right) \delta(\mathrm{ppm}) 157.1$ (s), 143.8 (d), 129.3 (d), 75.7 (d), 43.1 (d), 35.1 (t), 35.0 (t), 32.7 (d), 26.1 (d), 24.1 (d), 24.1 (t), 22.7 (q), 21.4 (q), 15.2 (q), -1.3 (q). IR $\left(\mathrm{CHCl}_{3}\right.$, $\left.\mathrm{cm}^{-1}\right) 3507,3324$ (br), 2913, 2869, 1730. LRMS ( $\mathrm{m} / \mathrm{z}$, relative intensity) $311\left(\mathrm{M}^{+}, 5\right), 235$ (35), 135 (87), 109 (89), 83 (100). HRMS calcd for $\mathrm{C}_{17} \mathrm{H}_{33} \mathrm{NO}_{2} \mathrm{Si}$ : 311.2280, found: 311.2276. $[\alpha]_{\mathrm{D}}^{20}=-12.6\left(c=1.78, \mathrm{CHCl}_{3}\right)$.

(R,E)-1-((1R,2S,5R)-2-Isopropyl-5-methylcyclohexyl)-3-(trimethylsilyl)allyl carbamate (21c)

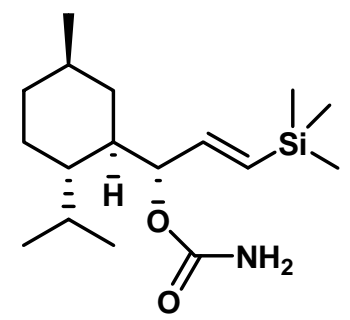

Prepared as per 11f from allylic alcohol 9c. The resulting light yellow solid was purified by flash chromatography ( $40 \%$ diethyl ether in hexanes) to yield pure $(R)$-allylcarbamate 21c (268 mg, 98\%) as a white solid. m.p.: $104-105{ }^{\circ} \mathrm{C} \quad \mathbf{1}_{\mathbf{H}} \mathbf{N M R}\left(300 \mathrm{MHz}, \mathrm{CDCl}_{3}\right) \delta(\mathrm{ppm})$ $5.95\left(\mathrm{dd}, 1 \mathrm{H}, J_{1}=19.0 \mathrm{~Hz}, J_{2}=4.7 \mathrm{~Hz}\right), 5.87(\mathrm{~d}, 1 \mathrm{H}, J=19.2 \mathrm{~Hz}), 5.40(\mathrm{t}, 1 \mathrm{H}, J=4.4 \mathrm{~Hz}$ ), $4.63(\mathrm{br} \mathrm{s}, 2 \mathrm{H}), 2.05\left(\mathrm{septd}, 1 \mathrm{H}, J_{1}=6.8 \mathrm{~Hz}, J_{2}=2.0 \mathrm{~Hz}\right), 1.81-1.58(\mathrm{~m}, 5 \mathrm{H}), 1.34-1.23(\mathrm{~m}$, $1 \mathrm{H}), 1.03-0.98(\mathrm{~m}, 2 \mathrm{H}), 0.87$ (d, $3 \mathrm{H}, J=7.2 \mathrm{~Hz}), 0.85$ (d, $3 \mathrm{H}, J=6.6 \mathrm{~Hz}), 0.83(\mathrm{~d}, 3 \mathrm{H}, J=$ $7.2 \mathrm{~Hz}), 0.68(\mathrm{q}, 1 \mathrm{H}, J=11.9 \mathrm{~Hz}), 0.07(\mathrm{~s}, 9 \mathrm{H}) .{ }^{13} \mathrm{C} \mathrm{NMR}\left(75.5 \mathrm{MHz}, \mathrm{CDCl}_{3}\right) \delta(\mathrm{ppm})$ 156.6 (s), 140.3 (d), 132.3 (d), 76.7 (d), 43.9 (d), 42.9 (d), 35.1 (t), 34.9 (t), 32.3 (d), 26.4 (d), 24.1 (t), 22.7 (q), 21.4 (q), 15.2 (q), -1.4 (q). IR $\left(\mathrm{CHCl}_{3}, \mathrm{~cm}^{-1}\right)$ 3510, 3346, 3270, 3182, 2954, 2921, 2871, 1727, 1711, 1599. LRMS ( $\mathrm{m} / \mathrm{z}$, relative intensity) $311\left(\mathrm{M}^{+}, 7\right)$, 135 (68), 118 (100), 83 (89). HRMS calcd for $\mathrm{C}_{17} \mathrm{H}_{33} \mathrm{NO}_{2} \mathrm{Si}$ : 311.2280, found: 311.2276. $[\alpha]_{\mathrm{D}}^{20}=-44.1\left(c=1.28, \mathrm{CHCl}_{3}\right)$. 
(S,E)-1-((1R,2S,5R)-2-Isopropyl-5-methylcyclohexyl)-3-methylhex-2-enyl carbamate (11d)

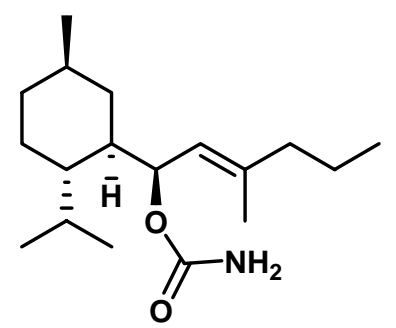

Prepared as per 11f from allylic alcohol 8d. The resulting oil was purified by flash chromatography (15\% ethyl acetate in hexanes) to yield pure $(S)$-allylcarbamate 11d (3.56 g, $>99 \%)$ as a colorless oil. ${ }^{1} \mathbf{H}$ NMR $\left(300 \mathrm{MHz}, \mathrm{CDCl}_{3}\right) \delta(\mathrm{ppm}) 5.59\left(\mathrm{dd}, 1 \mathrm{H}, J_{1}=8.8\right.$ $\mathrm{Hz}, J_{2}=2.2 \mathrm{~Hz}$ ), $5.24(\mathrm{~d}, 1 \mathrm{H}, J=8.8 \mathrm{~Hz}), 4.47(\mathrm{br} \mathrm{s}, 2 \mathrm{H}), 2.19$ (septd, $1 \mathrm{H}, J_{1}=6.9 \mathrm{~Hz}, J_{2}=$ $3.0 \mathrm{~Hz}), 1.99(\mathrm{t}, 2 \mathrm{H}, J=7.7 \mathrm{~Hz}), 1.79-1.62(\mathrm{~m}, 3 \mathrm{H}), 1.70(\mathrm{~s}, 3 \mathrm{H}), 1.50-1.38(\mathrm{~m}, 3 \mathrm{H}), 1.31-$ $1.12(\mathrm{~m}, 2 \mathrm{H}), 1.04-0.79(\mathrm{~m}, 6 \mathrm{H}), 0.90(\mathrm{~d}, 3 \mathrm{H}, J=7.2 \mathrm{~Hz}), 0.88(\mathrm{~d}, 3 \mathrm{H}, J=7.2 \mathrm{~Hz}), 0.77$ (d, $3 \mathrm{H}, J=6.6 \mathrm{~Hz}) .{ }^{13} \mathrm{C}$ NMR $\left(75.5 \mathrm{MHz}, \mathrm{CDCl}_{3}\right) \delta(\mathrm{ppm}) 157.3(\mathrm{~s}), 138.4$ (s), 122.6 (d), 72.1 (d), 43.9 (d), $43.1(\mathrm{~d}), 41.7(\mathrm{t}), 35.6(\mathrm{t}), 34.9(\mathrm{t}), 32.7$ (d), $26.2(\mathrm{~d}), 24.1(\mathrm{t}), 22.7(\mathrm{q})$, 21.6 (q), 20.6 (t), 16.5 (q), 15.3 (q), 13.5 (q) IR (neat, cm-1) 3514, 3333 (br), 2962, 2931, 2871, 1715, 1384, 1032. LRMS ( $\mathrm{m} / \mathrm{z}$, relative intensity) $295\left(\mathrm{M}^{+}, 3\right), 235((\mathrm{M}$ $\left.\left.\mathrm{OCONH}_{2}\right)^{+}, 15\right), 234\left(\left(\mathrm{M}-\mathrm{HOCONH}_{2}\right)^{+}, 73\right), 191$ (93), 138 (93), 123 (93), 113 (91), 96 (100), 81 (98). HRMS calcd for $\mathrm{C}_{18} \mathrm{H}_{33} \mathrm{NO}_{2}: 295.2511$, found: $295.2513 .[\alpha]_{\mathrm{D}}{ }^{20}=-12.3(c$ $\left.=1.17, \mathrm{CHCl}_{3}\right)$.

$(R, E)-1-((1 R, 2 S, 5 R)$-2-Isopropyl-5-methylcyclohexyl)-3-methylhex-2-enyl carbamate (21d)

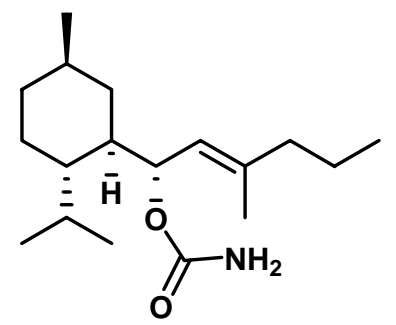

Prepared as per 11f from allylic alcohol 9d. The resulting light yellow powder was purified by flash chromatography ( $15 \%$ ethyl acetate in hexanes) to yield pure $(R)$-allylcarbamate 21d (426 mg, > 99\%) as a white powder. m.p.: $91-92{ }^{\circ} \mathrm{C} .{ }^{\mathbf{1}} \mathbf{H}$ NMR $\left(300 \mathrm{MHz}, \mathrm{CDCl}_{3}\right) \delta$ (ppm) $5.63\left(\mathrm{dd}, 1 \mathrm{H}, J_{1}=9.9 \mathrm{~Hz}, J_{2}=3.8\right), 5.26(\mathrm{~d}, 1 \mathrm{H}, J=9.9 \mathrm{~Hz}), 4.51(\mathrm{br} \mathrm{s}, 2 \mathrm{H}), 2.09-$ $1.94(\mathrm{~m}, 2 \mathrm{H}), 1.87-1.51(\mathrm{~m}, 5 \mathrm{H}), 1.73(\mathrm{~s}, 3 \mathrm{H}), 1.44(\mathrm{sept}, 2 \mathrm{H}, J=6.5 \mathrm{~Hz}), 1.35-1.25(\mathrm{~m}$, $1 \mathrm{H}), 1.08-0.68(\mathrm{~m}, 7 \mathrm{H}), 0.90(\mathrm{~d}, 3 \mathrm{H}, J=6.6 \mathrm{~Hz}), 0.85(\mathrm{~d}, 3 \mathrm{H}, J=6.6 \mathrm{~Hz}), 0.83(\mathrm{~d}, 3 \mathrm{H}, J=$ 6.6 Hz). ${ }^{13} \mathrm{C}$ NMR $\left(75.5 \mathrm{MHz}, \mathrm{CDCl}_{3}\right) \delta(\mathrm{ppm}) 156.8(\mathrm{~s}), 142.9(\mathrm{~s}), 119.1$ (d), $71.9(\mathrm{~d})$, $43.9(\mathrm{~d}), 42.5(\mathrm{~d}), 42.0(\mathrm{t}), 35.1(\mathrm{t}), 34.6(\mathrm{t}), 32.4(\mathrm{~d}), 26.5(\mathrm{~d}), 23.9(\mathrm{t}), 22.8(\mathrm{q}), 21.4(\mathrm{q})$, 20.7 (t), 16.6 (q), 15.2 (q), 13.5 (q). IR $\left(\mathrm{CHCl}_{3}, \mathrm{~cm}^{-1}\right)$ 3436, 3335, 3286, 3207, 3175, 2956, 2937, 2872, 1724, 1695, 1387, 1008. LRMS ( $\mathrm{m} / \mathrm{z}$, relative intensity) $295\left(\mathrm{M}^{+}, 2\right), 234((\mathrm{M}$ - $\left.\left.\mathrm{HOCONH}_{2}\right)^{+}, 62\right), 191$ (100), 138 (93), 123 (80), 113 (88), 95 (95), 81 (91). HRMS calcd for $\mathrm{C}_{18} \mathrm{H}_{33} \mathrm{NO}_{2}$ : 295.2511, found: 295.2513. $[\alpha]_{\mathrm{D}}{ }^{20}=-38.1\left(c=1.12, \mathrm{CHCl}_{3}\right)$. 
(S,E)-1-((1R,2S,5R)-2-Isopropyl-5-methylcyclohexyl)-3-phenylbut-2-enyl carbamate (11e)<smiles>CC1CCC(C(C)C)C(C(/C=C(\N)c2ccccc2)OC(N)=O)C1</smiles>

Prepared as per $11 \mathrm{f}$ from allylic alcohol 8e. The resulting light yellow solid was purified by flash chromatography (45\% diethyl ether in hexanes) to yield pure (S)-allylcarbamate 11e (398 $\mathrm{mg}, 90 \%)$ as a colorless oil. ${ }^{\mathbf{1}} \mathbf{H} \mathbf{~ N M R}\left(300 \mathrm{MHz}, \mathrm{CDCl}_{3}\right) \delta(\mathrm{ppm}) 7.42(\mathrm{~d}, 2 \mathrm{H}, J=$ $1.7 \mathrm{~Hz}), 7.41-7.23(\mathrm{~m}, 3 \mathrm{H}), 5.84(\mathrm{~d}, 1 \mathrm{H}, J=8.8 \mathrm{~Hz}), 5.77\left(\mathrm{dd}, 1 \mathrm{H}, J_{1}=8.3 \mathrm{~Hz}, J_{2}=2.2\right.$ $\mathrm{Hz}), 4.54$ (br s, 2H), 2.22 (septd, $\left.1 \mathrm{H}, J_{1}=7.2 \mathrm{~Hz}, J_{2}=2.9 \mathrm{~Hz}\right), 2.14(\mathrm{~s}, 3 \mathrm{H}), 1.82(\mathrm{dq}, 1 \mathrm{H}$, $\left.J_{1}=5.5 \mathrm{~Hz}, J_{2}=3.3 \mathrm{~Hz}\right), 1.73-1.66(\mathrm{~m}, 2 \mathrm{H}), 1.62-1.52(\mathrm{~m}, 2 \mathrm{H}), 1.43-1.20(\mathrm{~m}, 2 \mathrm{H}), 1.07-$ $0.75(\mathrm{~m}, 2 \mathrm{H}), 0.93(\mathrm{~d}, 3 \mathrm{H}, J=7.2 \mathrm{~Hz}), 0.89$ (d, $3 \mathrm{H}, J=6.6 \mathrm{~Hz}), 0.82(\mathrm{~d}, 3 \mathrm{H}, J=6.6 \mathrm{~Hz})$. ${ }^{13} \mathrm{C}$ NMR $\left(75.5 \mathrm{MHz}, \mathrm{CDCl}_{3}\right) \delta(\mathrm{ppm}) 157.2$ (s), 143.1 (s), 137.0 (s), 128.2 (d), 127.2 (d), 126.4 (d), 125.9 (d), 72.3 (d), 44.1 (d), 43.2 (d), 35.7 (t), 35.1 (t), 32.8 (d), 26.4 (d), 24.2 (t), 22.8 (q), 21.7 (q), 16.5 (q), 15.5 (q). IR (neat, $\mathrm{cm}^{-1}$ ) 3507, 3342, 2955, 2921, 2871, 1721. LRMS ( $\mathrm{m} / \mathrm{z}$, relative intensity) $329\left(\mathrm{M}^{+}, 3\right), 147$ (100), 130 (76), 95 (66), 84 (93). HRMS calcd for $\mathrm{C}_{21} \mathrm{H}_{31} \mathrm{NO}_{2}: 329.2355$, found: $329.2363 .[\alpha]_{\mathrm{D}}{ }^{20}=-2.3(c=1.21$, $\left.\mathrm{CHCl}_{3}\right)$.

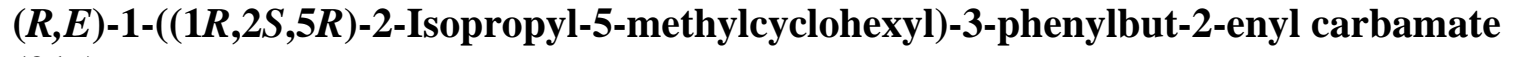
(21e)<smiles>CC1CCC(C(C)C)C(/C=C(\N)c2ccccc2)C1</smiles>

Prepared as per 11f from allylic alcohol 9e. The resulting light yellow solid was purified by flash chromatography ( $45 \%$ diethyl ether in hexanes) to yield pure $(R)$-allylcarbamate 21e (175 mg, 89\%) as a white solid. m.p.: $118-120{ }^{\circ} \mathrm{C} \quad \mathbf{1}_{\mathbf{H}} \mathbf{~ N M R}\left(300 \mathrm{MHz}, \mathrm{CDCl}_{3}\right) \delta(\mathrm{ppm})$ $7.43(\mathrm{~d}, 2 \mathrm{H}, J=1.7 \mathrm{~Hz}), 7.42-7.25(\mathrm{~m}, 3 \mathrm{H}), 5.86-5.77(\mathrm{~m}, 2 \mathrm{H}), 4.79(\mathrm{~s}, 2 \mathrm{H}), 2.18(\mathrm{~s}, 3 \mathrm{H})$, 1.98-1.81 (m, 3H), 1.74-1.63 (m, 2H), 1.44-1.27 (m, 1H), 1.07-0.76 (m, 4H), 0.92 (d, 3H, J $=6.6 \mathrm{~Hz}), 0.89(\mathrm{~d}, 3 \mathrm{H}, J=7.2 \mathrm{~Hz}), 0.87(\mathrm{~d}, 3 \mathrm{H}, J=7.2 \mathrm{~Hz}) . \quad$ IR $\left(\mathrm{CHCl}_{3}, \mathrm{~cm}^{-1}\right) 3500$, 3335, 3177, 2953, 2919, 2867, 1713. LRMS ( $\mathrm{m} / \mathrm{z}$, relative intensity) $329\left(\mathrm{M}^{+}, 2\right), 225(67)$, 147 (100), 129 (98), 95 (90), 81 (62). HRMS calcd for $\mathrm{C}_{21} \mathrm{H}_{31} \mathrm{NO}_{2}$ : 329.2355, found: 329.2366. $[\alpha]_{\mathrm{D}}^{20}=-23.3\left(c=1.04, \mathrm{CHCl}_{3}\right)$. 


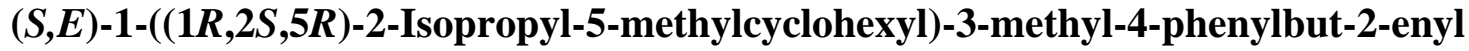
carbamate (11f)<smiles>C/C(=C\[C@H](OC(N)=O)C1C[C@@H](C)CC[C@H]1C(C)C)Cc1ccccc1</smiles>

The alcohol 8f $(510 \mathrm{mg}, 1.7 \mathrm{mmol})$ was dissolved in dichloromethane $(14 \mathrm{~mL})$, and the solution was cooled to $0{ }^{\circ} \mathrm{C}$. Trichloroacetylisocyanate $(0.30 \mathrm{~mL}, 2.5 \mathrm{mmol})$ was then added dropwise. The solution was stirred for $1 \mathrm{~h}$ at $0{ }^{\circ} \mathrm{C}$. The solvent was then evaporated under reduced pressure and the resulting precipitate was dissolved in a mixture of methanol $(11.3 \mathrm{~mL})$ and water $(5.6 \mathrm{~mL})$. This solution was cooled to $0{ }^{\circ} \mathrm{C}$ and potassium carbonate (700 mg, $5.1 \mathrm{mmol}$ ) was slowly added. The mixture was allowed to warm slowly to $23^{\circ} \mathrm{C}$ and was stirred for $15 \mathrm{~h}$. Methanol was then evaporated under reduced pressure and the aqueous phase was extracted with $3 \times 15 \mathrm{~mL}$ of dichloromethane. The organic layers were combined, washed with brine, dried with $\mathrm{MgSO}_{4}$, filtered and concentrated under reduced pressure. The resulting oil was purified by flash chromatography (20\% ethyl acetate in hexanes) to yield pure (S)-allylcarbamate $\mathbf{1 1 f}(578 \mathrm{mg}, 99 \%)$ as a colorless oil. ${ }^{1} \mathbf{H}$ NMR $\left(300 \mathrm{MHz}, \mathrm{CDCl}_{3}\right) \delta(\mathrm{ppm}) 7.31-7.15(\mathrm{~m}, 5 \mathrm{H}), 5.60\left(\mathrm{dd}, 1 \mathrm{H}, J_{1}=8.5 \mathrm{~Hz}, J_{2}=2.5 \mathrm{~Hz}\right)$, $5.34(\mathrm{~d}, 1 \mathrm{H}, J=8.2 \mathrm{~Hz}$ ), 4.50 (br s, $2 \mathrm{H}), 3.33$ (AB quartet, $2 \mathrm{H}$ ), 2.14 (septd, $1 \mathrm{H}, J_{1}=6.9$ $\left.\mathrm{Hz}, J_{2}=3.1 \mathrm{~Hz}\right), 1.62-1.80(\mathrm{~m}, 3 \mathrm{H}), 1.66(\mathrm{~s}, 3 \mathrm{H}), 1.53-1.44(\mathrm{~m}, 1 \mathrm{H}), 1.33-1.13(\mathrm{~m}, 3 \mathrm{H})$, $1.00-0.76(\mathrm{~m}, 2 \mathrm{H}), 0.89$ (d, $3 \mathrm{H}, J=6.6 \mathrm{~Hz}), 0.88(\mathrm{~d}, 3 \mathrm{H}, J=7.2 \mathrm{~Hz}), 0.75(\mathrm{~d}, 3 \mathrm{H}, \mathrm{J}=6.6$ Hz). ${ }^{13} \mathrm{C} \mathrm{NMR}\left(75.5 \mathrm{MHz}, \mathrm{CDCl}_{3}\right) \delta(\mathrm{ppm}) 156.9$ (s), 139.6 (s), 137.7 (s), 128.9 (d), 128.3 (d), 126.1 (d), 124.9 (d), 72.3 (d), $46.1(\mathrm{t}), 44.0$ (d), $43.2(\mathrm{~d}), 35.8(\mathrm{t}), 35.1(\mathrm{t}), 32.8$ (d), 26.3 (d), 24.1 (t), 22.8 (q), 21.6 (q), 16.6 (q), 15.4 (q). IR (neat, $\mathrm{cm}^{-1}$ ) 3502, 3333, 2955, 2920, 1718, 1370, 1031. LRMS ( $m / z$, relative intensity, CI: $\left.\mathrm{NH}_{3}\right) 361\left(\mathrm{MNH}_{4}{ }^{+}, 11\right)$ $344\left(\mathrm{MH}^{+}, 6\right), 300\left(\left(\mathrm{MNH}_{4}-\mathrm{HOCONH}_{2}\right)^{+}, 55\right), 283\left(\left(\mathrm{MH}-\mathrm{HOCONH}_{2}\right)^{+}, 100\right)$ HRMS calcd for $\mathrm{C}_{22} \mathrm{H}_{34} \mathrm{NO}_{2}\left(\mathrm{MH}^{+}\right): 344.2589$, found: 344.2575 . $[\alpha]_{\mathrm{D}}{ }^{20}=-29.1\left(c=1.01, \mathrm{CHCl}_{3}\right)$.

\section{(R,E)-1-((1R,2S,5R)-2-Isopropyl-5-methylcyclohexyl)-3-methyl-4-phenylbut-2-enyl carbamate (21f)}

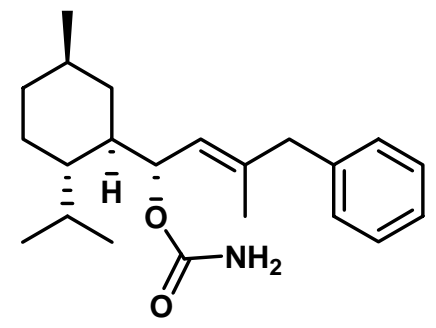

Prepared as per 11f from allylic alcohol 9f. The resulting oil was purified by flash chromatography ( $20 \%$ ethyl acetate in hexanes) to yield pure $(R)$-allylcarbamate 21 (125 $\mathrm{mg}, 88 \%)$ as a white powder. m.p.: $102-103{ }^{\circ} \mathrm{C} \mathbf{1}_{\mathbf{H}} \mathbf{~ N M R}\left(300 \mathrm{MHz}, \mathrm{CDCl}_{3}\right) \delta(\mathrm{ppm})$ 7.30-7.22 (m, 2H), 7.19 (d, 1H, $J=7.7 \mathrm{~Hz}$ ), $7.15(\mathrm{~d}, 2 \mathrm{H}, J=7.2 \mathrm{~Hz}), 5.64\left(\mathrm{dd}, 1 \mathrm{H}, J_{1}=9.6\right.$ $\left.\mathrm{Hz}, J_{2}=4.1 \mathrm{~Hz}\right), 5.43(\mathrm{~d}, 1 \mathrm{H}, J=10.5 \mathrm{~Hz}), 4.52(\mathrm{br} \mathrm{s}, 2 \mathrm{H}), 3.36$ (AB quartet, 2H), 1.89$1.62(\mathrm{~m}, 5 \mathrm{H}), 1.68(\mathrm{~s}, 3 \mathrm{H}), 1.34-1.25(\mathrm{~m}, 2 \mathrm{H}), 1.06-0.81(\mathrm{~m}, 3 \mathrm{H}), 0.91(\mathrm{~d}, 3 \mathrm{H}, J=6.6 \mathrm{~Hz})$, 
$0.84(\mathrm{~d}, 3 \mathrm{H}, J=6.6 \mathrm{~Hz}), 0.82(\mathrm{~d}, 3 \mathrm{H}, J=7.1 \mathrm{~Hz}) . \mathbf{I R}\left(\mathrm{CHCl}_{3}, \mathrm{~cm}^{-1}\right) \quad 3505,3332,3263$, 2953, 2920, 2870, 1718, 1384, 1355, 1026. LRMS ( $\mathrm{m} / \mathrm{z}$, relative intensity, CI: $\left.\mathrm{NH}_{3}\right) 361$ $\left(\mathrm{MNH}_{4}{ }^{+}, 9\right) 344\left(\mathrm{MH}^{+}, 4\right), 300\left(\left(\mathrm{MNH}_{4}-\mathrm{HOCONH}_{2}\right)^{+}, 62\right), 283\left(\left(\mathrm{MH}-\mathrm{HOCONH}_{2}\right)^{+}, 100\right)$ HRMS calcd for $\mathrm{C}_{22} \mathrm{H}_{34} \mathrm{NO}_{2}: 344.2589$, found: 344.2596 . $[\alpha]_{\mathrm{D}}^{20}=2.6\left(c=1.01, \mathrm{CHCl}_{3}\right)$.

\section{Formation of allylisocyanates}

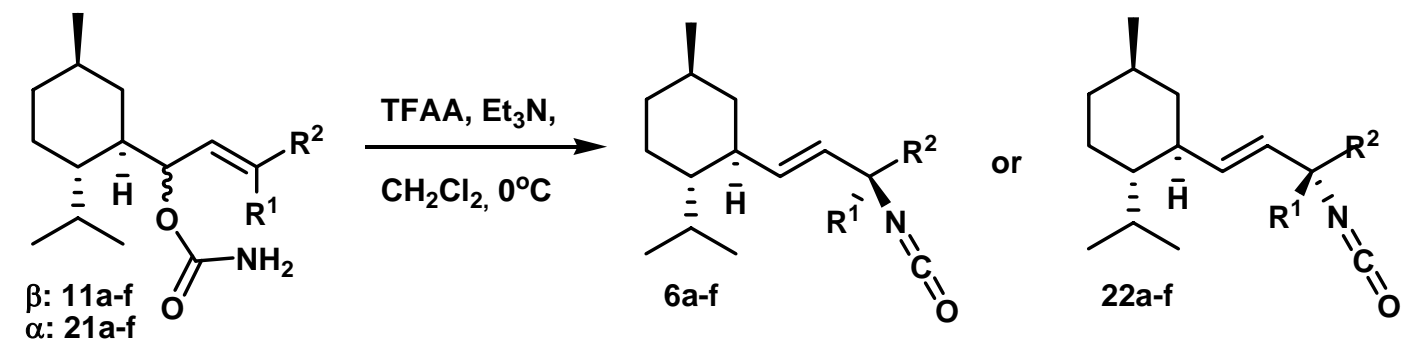

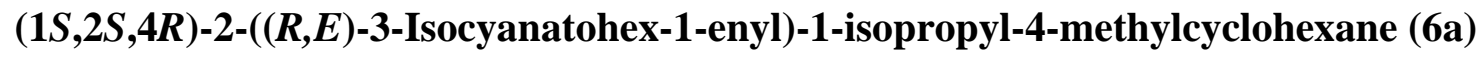<smiles>CCCC(/C=C/C1CC(C)CCC1C(C)C)N=C=O</smiles>

Prepared as per 6d from allylcarbamate 11a. Gave a colorless oil as the pure $(R)$ allylisocyanate 6a (47 mg, 96\%). > 99\%de (measured by gas chromatography). ${ }^{\mathbf{1}} \mathbf{H}$ NMR $\left(300 \mathrm{MHz}, \mathrm{CDCl}_{3}\right) \delta(\mathrm{ppm}) 5.43\left(\mathrm{dd}, 1 \mathrm{H}, J_{1}=15.1 \mathrm{~Hz}, J_{2}=8.5 \mathrm{~Hz}\right), 5.34\left(\mathrm{dd}, 1 \mathrm{H}, J_{1}=\right.$ $\left.14.8 \mathrm{~Hz}, J_{2}=6.1 \mathrm{~Hz}\right), 3.92(\mathrm{q}, 1 \mathrm{H}, J=6.4 \mathrm{~Hz}), 1.95-1.84(\mathrm{~m}, 1 \mathrm{H}), 1.80-1.70(\mathrm{~m}, 2 \mathrm{H}), 1.64-$ $1.50(\mathrm{~m}, 4 \mathrm{H}), 1.48-1.31(\mathrm{~m}, 3 \mathrm{H}), 1.02-0.79(\mathrm{~m}, 4 \mathrm{H}), 0.93(\mathrm{t}, 3 \mathrm{H}, J=7.2 \mathrm{~Hz}), 0.87(\mathrm{~d}, 6 \mathrm{H}, J$ $=6.6 \mathrm{~Hz}), 0.69(\mathrm{~d}, 3 \mathrm{H}, J=7.2 \mathrm{~Hz}) .{ }^{13} \mathrm{C} \mathbf{N M R}\left(75.5 \mathrm{MHz}, \mathrm{CDCl}_{3}\right) \delta(\mathrm{ppm}) 137.0(\mathrm{~d})$, $128.8(\mathrm{~d}), 109.5(\mathrm{~s}), 57.1(\mathrm{~s}), 47.0(\mathrm{~d}), 44.2(\mathrm{~d}), 43.0(\mathrm{t}), 39.7(\mathrm{t}), 35.1(\mathrm{t}), 32.4(\mathrm{~d}), 28.2(\mathrm{~d})$, 24.0 (t), 22.5 (q), 21.4 (q), 19.0 (t), 15.2 (q), 13.5 (q). IR (neat, $\left.\mathrm{cm}^{-1}\right)$ 2956, 2928, 2871, 2261, 1461, 969. LRMS $\left(\mathrm{m} / \mathrm{z}\right.$, relative intensity) $263\left(\mathrm{M}^{+}, 35\right), 220\left(\left(\mathrm{M}-\mathrm{C}_{3} \mathrm{H}_{7}\right)^{+}, 85\right), 138$ (79), 109 (70), 95 (100), 81 (80). HRMS calcd for $\mathrm{C}_{17} \mathrm{H}_{29} \mathrm{NO}$ : 263.2249, found: 263.2257. $[\alpha]_{\mathrm{D}}^{20}=-100.5\left(c=1.01, \mathrm{CHCl}_{3}\right)$.

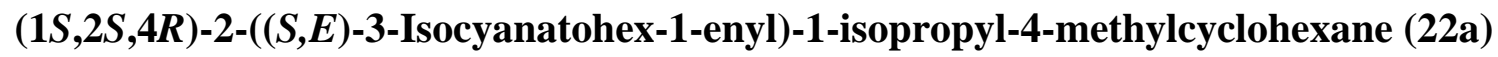<smiles>CCCC(/C=C/C1CC(C)CCC1C(C)C)N=C=O</smiles>

Prepared as per 6d from allylcarbamate 21a. Gave a colorless oil as the pure $(S)$ allylisocyanate 22a (26 mg, 96\%). 99\% de (measured by gas chromatography). ${ }^{\mathbf{1}} \mathbf{H}$ NMR $\left(300 \mathrm{MHz}, \mathrm{CDCl}_{3}\right) \delta(\mathrm{ppm}) 5.44\left(\mathrm{dd}, 1 \mathrm{H}, J_{1}=15.4 \mathrm{~Hz}, J_{2}=8.2 \mathrm{~Hz}\right), 5.35\left(\mathrm{dd}, 1 \mathrm{H}, J_{1}=\right.$ $\left.14.8 \mathrm{~Hz}, J_{2}=6.1 \mathrm{~Hz}\right), 3.92(\mathrm{q}, 1 \mathrm{H}, J=6.2 \mathrm{~Hz}), 1.96-1.66(\mathrm{~m}, 3 \mathrm{H}), 1.64-1.50(\mathrm{~m}, 4 \mathrm{H}), 1.48-$ 
$1.25(\mathrm{~m}, 3 \mathrm{H}), 1.03-0.82(\mathrm{~m}, 4 \mathrm{H}), 0.92(\mathrm{t}, 3 \mathrm{H}, J=7.4 \mathrm{~Hz}), 0.88(\mathrm{~d}, 3 \mathrm{H}, J=7.2 \mathrm{~Hz}), 0.87$ (d, $3 \mathrm{H}, J=6.6 \mathrm{~Hz}), 0.71(\mathrm{~d}, 3 \mathrm{H}, J=6.6 \mathrm{~Hz}) .{ }^{13} \mathrm{C}$ NMR $\left(75.5 \mathrm{MHz}, \mathrm{CDCl}_{3}\right) \delta(\mathrm{ppm}) 136.9$ (d), $128.8(\mathrm{~d}), 110.1(\mathrm{~s}), 57.1$ (d), $47.1(\mathrm{~d}), 44.1(\mathrm{~d}), 43.0(\mathrm{t}), 39.6(\mathrm{t}), 35.1(\mathrm{t}), 32.4(\mathrm{~d})$, 28.1 (d), 24.0 (t), 22.5 (q), 21.3 (q), 19.0 (t), 15.3 (q), 13.6 (q). IR (neat, $\mathrm{cm}^{-1}$ ) 2957, 2927, 2872, 2258. LRMS $\left(\mathrm{m} / \mathrm{z}\right.$, relative intensity) $263\left(\mathrm{M}^{+}, 27\right), 220\left(\left(\mathrm{M}^{+}-\mathrm{C}_{3} \mathrm{H}_{7}\right)^{+}, 71\right), 138$ (63), 109 (60), 95 (100), 82 (87). HRMS calcd for $\mathrm{C}_{17} \mathrm{H}_{29} \mathrm{NO}$ : 263.2249, found: 263.2257. $[\alpha]_{\mathrm{D}}^{20}=-38.5\left(c=0.87, \mathrm{CHCl}_{3}\right)$.

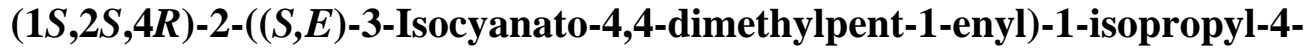 methylcyclohexane (6b)}

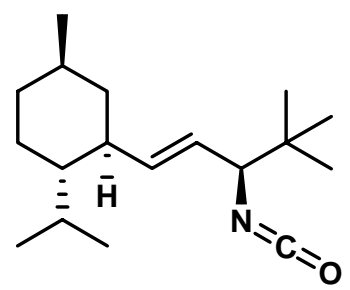

Prepared as per 6d from allylcarbamate 11b. The resulting light yellow oil was purified by flash chromatography (100\% hexanes) to yield pure (S)-allylisocyanate $\mathbf{6 b}(43 \mathrm{mg}, 93 \%)$ as a colorless oil. 99\% de (measured by gas chromatography). ${ }^{1} \mathbf{H}$ NMR $\left(300 \mathrm{MHz}, \mathrm{CDCl}_{3}\right) \delta$ (ppm) $5.43-5.41(\mathrm{~m}, 2 \mathrm{H}), 3.62(\mathrm{~d}, 1 \mathrm{H}, J=7.15 \mathrm{~Hz}), 1.98-1.82(\mathrm{~m}, 1 \mathrm{H}), 1.80-1.70(\mathrm{~m}, 2 \mathrm{H})$, $1.66-1.55(\mathrm{~m}, 2 \mathrm{H}), 1.43-1.29(\mathrm{~m}, 1 \mathrm{H}), 1.11-0.77(\mathrm{~m}, 4 \mathrm{H}), 0.91(\mathrm{~s}, 9 \mathrm{H}), 0.89(\mathrm{~d}, 3 \mathrm{H}, J=7.7$ $\mathrm{Hz}), 0.87(\mathrm{~d}, 3 \mathrm{H}, J=6.05 \mathrm{~Hz}), 0.69$ (d, 3H, $J=7.15 \mathrm{~Hz}) .{ }^{13} \mathrm{C}$ NMR $\left(75.5 \mathrm{MHz}, \mathrm{CDCl}_{3}\right) \delta$ (ppm) 139.0 (d), 125.9 (d), 123.1 (s), 67.5 (d), 47.0 (d), 44.7 (d), $43.2(\mathrm{t}), 35.3(\mathrm{~s}), 35.1(\mathrm{t})$, 32.4 (d), 28.3 (d), 26.1 (q), 23.9 (t), 22.5 (q), 21.4 (q), 15.0 (q). IR (neat, $\mathrm{cm}^{-1}$ ) 2957, 2871, 2845, 2252, 1458, 1366. LRMS $\left(\mathrm{m} / \mathrm{z}\right.$, relative intensity) $277\left(\mathrm{M}^{+}, 7\right), 220\left(\left(\mathrm{M}-\mathrm{C}_{4} \mathrm{H}_{9}\right)^{+}\right.$, 100). HRMS calcd for $\mathrm{C}_{18} \mathrm{H}_{31} \mathrm{NO}: 277.2405$, found: 277.2410 . $[\alpha]_{\mathrm{D}}{ }^{20}=-119.4(c=1.11$, $\left.\mathrm{CHCl}_{3}\right)$.

(1S,2S,4R)-2-((R,E)-3-Isocyanato-4,4-dimethylpent-1-enyl)-1-isopropyl-4methylcyclohexane (22b)

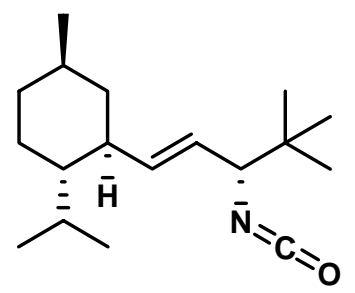

Prepared as per 6d from allylcarbamate 21b. The resulting light yellow oil was purified by flash chromatography (100\% hexanes) to yield pure $(R)$-allylisocyanate $22 \mathbf{b}$ (38 $\mathrm{mg}, 88 \%)$ as a colorless oil. 99\% de (measured by gas chromatography). ${ }^{\mathbf{1}} \mathbf{H} \mathbf{~ N M R ~}(300 \mathrm{MHz}$, $\left.\mathrm{CDCl}_{3}\right) \delta(\mathrm{ppm}) 5.44-5.42(\mathrm{~m}, 2 \mathrm{H}), 3.64\left(\mathrm{dd}, 1 \mathrm{H}, J_{1}=4.4 \mathrm{~Hz}, J_{2}=1.65 \mathrm{~Hz}\right), 1.99-1.90(\mathrm{~m}$, $1 \mathrm{H}), 1.83$ (septd, $\left.1 \mathrm{H}, J_{1}=7.15 \mathrm{~Hz}, J_{2}=2.2 \mathrm{~Hz}\right), 1.74-1.66(\mathrm{~m}, 1 \mathrm{H}), 1.64-1.40(\mathrm{~m}, 2 \mathrm{H})$, $1.39-1.25(\mathrm{~m}, 1 \mathrm{H}), 1.03-0.96(\mathrm{~m}, 1 \mathrm{H}), 0.96-0.80(\mathrm{~m}, 3 \mathrm{H}), 0.91(\mathrm{~s}, 9 \mathrm{H}), 0.88(\mathrm{~d}, 3 \mathrm{H}, J=$ $7.15 \mathrm{~Hz}), 0.87(\mathrm{~d}, 3 \mathrm{H}, J=6.6), 0.72(\mathrm{~d}, 3 \mathrm{H}, J=7.15 \mathrm{~Hz}) .{ }^{13} \mathrm{C}$ NMR $\left(75.5 \mathrm{MHz}, \mathrm{CDCl}_{3}\right) \delta$ (ppm) 138.7 (d), 125.8 (d), 109.9 (s), 67.4 (d), 47.0 (d), 44.5 (d), 43.1 (t), 35.4 (s), 35.1 (t), 
32.4 (d), 28.2 (d), 26.1 (q), 23.9 (t), 22.5 (q), 21.3 (q), 15.1 (q). IR (neat, $\mathrm{cm}^{-1}$ ) 2957, 2918, 2871, 2253, 1464, 1366. LRMS $\left(\mathrm{m} / \mathrm{z}\right.$, relative intensity) $277\left(\mathrm{M}^{+}, 5\right), 220\left(\left(\mathrm{M}_{-} \mathrm{C}_{4} \mathrm{H}_{9}\right)^{+}\right.$, (72)), $82(100)$. HRMS calcd for $\mathrm{C}_{18} \mathrm{H}_{31} \mathrm{NO}: 277.2405$, found: $277.2410 .[\alpha]_{\mathrm{D}}{ }^{20}=-24.2(\mathrm{c}$ $\left.=0.90, \mathrm{CHCl}_{3}\right)$.

((R,E)-1-Isocyanato-3-((1S,2S,5R)-2-isopropyl-5-methylcyclohexyl)allyl)trimethylsilane (6c)

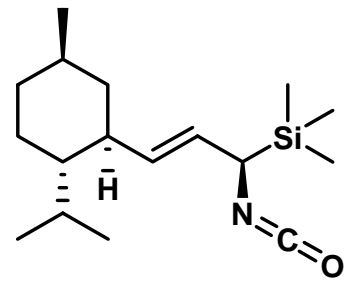

Prepared as per $\mathbf{6 d}$ from allylcarbamate 11c. The resulting light yellow oil was purified by flash chromatography ( $100 \%$ hexanes) to yield pure $(R)$-allylisocyanate $6 c$ ( $419 \mathrm{mg}, 93 \%)$ as a colorless oil. $>99 \%$ de (measured by HPLC). ${ }^{\mathbf{1}} \mathbf{H}$ NMR $\left(300 \mathrm{MHz}, \mathrm{CDCl}_{3}\right) \delta(\mathrm{ppm})$ 5.39-5.25 (m, 2H), $3.49(\mathrm{~d}, 1 \mathrm{H}, J=5.0 \mathrm{~Hz}), 1.95-1.66(\mathrm{~m}, 3 \mathrm{H}), 1.63-1.54(\mathrm{~m}, 2 \mathrm{H}), 1.43-$ $1.31(\mathrm{~m}, 1 \mathrm{H}), 1.01-0.72(\mathrm{~m}, 4 \mathrm{H}), 0.87(\mathrm{~d}, 6 \mathrm{H}, J=6.6 \mathrm{~Hz}), 0.69$ (d, 3H, $J=7.2 \mathrm{~Hz}), 0.09$ (s, 9H). ${ }^{13} \mathrm{C}$ NMR $\left(75.5 \mathrm{MHz}, \mathrm{CDCl}_{3}\right) \delta$ (ppm) 133.9 (d), 125.0 (d), 121.6 (s), 48.3 (d), 47.1 (d), 44.6 (d), 43.5 (t), 35.1 (t), 32.5 (d), 28.0 (d), 23.9 (t), 22.5 (q), 21.4 (q), 15.1 (q), -4.1 (q). IR (neat, $\left.\mathrm{cm}^{-1}\right)$ 2956, 2917, 2871, 2265, 1251. LRMS ( $\mathrm{m} / \mathrm{z}$, relative intensity) $293\left(\mathrm{M}^{+}\right.$, 5), 250 ((M-HCNO) $\left.)^{+}, 43\right), 155$ (100), 139 (50). HRMS calcd for $\mathrm{C}_{17} \mathrm{H}_{31} \mathrm{NOSi}$ : 293.2175, found: $293.2165 .[\alpha]_{\mathrm{D}}^{20}=-111.1\left(c=1.31, \mathrm{CHCl}_{3}\right)$.

((S,E)-1-Isocyanato-3-((1S,2S,5R)-2-isopropyl-5-methylcyclohexyl)allyl)trimethylsilane (22c)

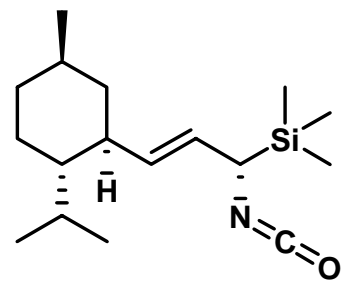

Prepared as per 6d from allylcarbamate 21c. The resulting light yellow oil was purified by flash chromatography (100\% hexanes) to yield pure (S)-allylisocyanate 22c (114 mg, 94\%) as a colorless oil. 94\%de (measured by HPLC). ${ }^{1} \mathbf{H}$ NMR $\left(300 \mathrm{MHz}, \mathrm{CDCl}_{3}\right) \delta(\mathrm{ppm})$ $5.38\left(\mathrm{dd}, 1 \mathrm{H}, J_{1}=15.1 \mathrm{~Hz}, J_{2}=5.2 \mathrm{~Hz}\right), 5.29\left(\mathrm{dd}, 1 \mathrm{H}, J_{1}=15.7 \mathrm{~Hz}, J_{2}=8.5 \mathrm{~Hz}\right), 3.50(\mathrm{~d}$, $1 \mathrm{H}, J=5.0 \mathrm{~Hz}), 1.98-1.79(\mathrm{~m}, 2 \mathrm{H}), 1.74-1.70(\mathrm{~m}, 1 \mathrm{H}), 1.64-1.52(\mathrm{~m}, 2 \mathrm{H}), 1.43-1.31(\mathrm{~m}$, $1 \mathrm{H}), 1.01-0.81(\mathrm{~m}, 4 \mathrm{H}), 0.88(\mathrm{~d}, 3 \mathrm{H}, J=7.2 \mathrm{~Hz}), 0.86$ (d, 3H, $J=6.6 \mathrm{~Hz}), 0.70(\mathrm{~d}, 3 \mathrm{H}, J=$ $7.2 \mathrm{~Hz}), 0.09$ (s, 9H). ${ }^{13} \mathrm{C}$ NMR (75.5 MHz, $\left.\mathrm{CDCl}_{3}\right) \delta(\mathrm{ppm}) 133.4$ (d), 125.1 (d), 121.5 (s), 48.3 (d), 47.1 (d), 44.7 (d), 43.5 (t), 35.1 (t), 32.5 (d), 28.0 (d), 23.9 (t), 22.5 (q), 21.4 (q), 15.1 (q), -4.0 (q). IR (neat, $\left.\mathrm{cm}^{-1}\right)$ 2956, 2915, 2871, 2254, 1252. LRMS ( $\mathrm{m} / \mathrm{z}$, relative intensity) $293\left(\mathrm{M}^{+}, 5\right), 250\left((\mathrm{M}-\mathrm{HCNO})^{+}, 35\right), 155$ (60), 139 (50), 73 (100). HRMS calcd for $\mathrm{C}_{17} \mathrm{H}_{31} \mathrm{NOSi}$ : 293.2175, found: $293.2168 .[\alpha]_{\mathrm{D}}^{20}=-13.8\left(c=1.12, \mathrm{CHCl}_{3}\right)$. 


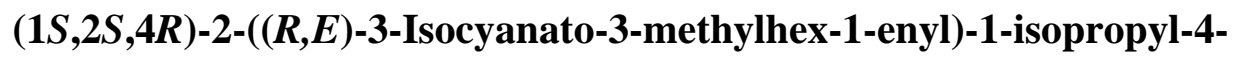
methylcyclohexane (6d)

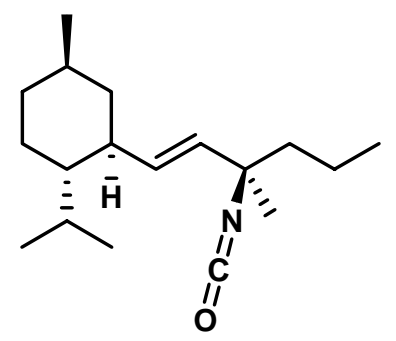

To a cold solution $\left(0{ }^{\circ} \mathrm{C}\right)$ of allylcarbamate $11 \mathrm{~d}(46 \mathrm{mg}, 0.16 \mathrm{mmol})$ in dichloromethane $(1.2 \mathrm{~mL}, 0.13 \mathrm{M})$ was added triethylamine $(65 \mu \mathrm{L}, 0.47 \mathrm{mmol})$. Then, trifluoroacetic anhydride $(23 \mu \mathrm{l}, 0.16 \mathrm{mmol})$ freshly distilled over $\mathrm{P}_{2} \mathrm{O}_{5}$ was added dropwise to the solution at $0{ }^{\circ} \mathrm{C}$. After stirring at $0{ }^{\circ} \mathrm{C}$ for $15 \mathrm{~min}$, the reaction mixture was poured slowly over an aqueous saturated solution of $\mathrm{NH}_{4} \mathrm{Cl}$ and it was extracted three times with diethyl ether $(10 \mathrm{~mL})$. The organic layers were combined, washed with brine, dried with $\mathrm{MgSO}_{4}$, filtered and concentrated under reduced pressure to give a light yellow oil as the pure $(R)$ allylisocyanate $\mathbf{6 d}$ (43 mg, > 99\%). 99\%de (measured by gas chromatography). ${ }^{\mathbf{1}} \mathbf{H}$ NMR $\left(300 \mathrm{MHz}, \mathrm{CDCl}_{3}\right) \delta(\mathrm{ppm}) 5.42\left(\mathrm{dd}, 1 \mathrm{H}, J_{1}=15.4 \mathrm{~Hz}, J_{2}=8.8 \mathrm{~Hz}\right), 5.31(\mathrm{~d}, 1 \mathrm{H}, J=15.4$ $\mathrm{Hz}), 1.93-1.70(\mathrm{~m}, 3 \mathrm{H}), 1.64-1.52(\mathrm{~m}, 4 \mathrm{H}) 1.49-1.26(\mathrm{~m}, 4 \mathrm{H}), 1.36(\mathrm{~s}, 3 \mathrm{H}), 1.02-0.81(\mathrm{~m}$, $6 \mathrm{H}), 0.92(\mathrm{~d}, 3 \mathrm{H}, J=7.2 \mathrm{~Hz}), 0.87(\mathrm{~d}, 3 \mathrm{H}, J=6.6 \mathrm{~Hz}), 0.69(\mathrm{~d}, 3 \mathrm{H}, J=6.6 \mathrm{~Hz}) .{ }^{13} \mathrm{C}$ NMR $\left(75.5 \mathrm{MHz}, \mathrm{CDCl}_{3}\right) \delta(\mathrm{ppm}) 133.6(\mathrm{~d}), 133.1$ (d), $110.0(\mathrm{~s}), 61.6(\mathrm{~s}), 47.1(\mathrm{~d}), 45.7(\mathrm{t}), 44.2$ (d), $43.2(\mathrm{t}), 35.1(\mathrm{t}), 32.4(\mathrm{~d}), 29.4(\mathrm{q}), 28.1(\mathrm{~d}), 24.1(\mathrm{t}), 22.5(\mathrm{q}), 21.3(\mathrm{q}), 17.6(\mathrm{t}), 15.3$ (q), 14.1 (q). IR (neat, $\mathrm{cm}^{-1}$ ) 2957, 2929, 2873, 2260, 1455, 972. LRMS ( $\mathrm{m} / \mathrm{z}$, relative intensity) $277\left(\mathrm{M}^{+}, 5\right), 234\left((\mathrm{M}-\mathrm{HNCO})^{+}, 58\right), 191$ (38), 96 (100). HRMS calcd for $\mathrm{C}_{18} \mathrm{H}_{31} \mathrm{NO}$ : 277.2405, found: 277.2399. $[\alpha]_{\mathrm{D}}^{20}=-73.4\left(c=1.23, \mathrm{CHCl}_{3}\right)$.

\section{$(1 S, 2 S, 4 R)-2-((S, E)-3-I s o c y a n a t o-3-m e t h y l h e x-1-e n y l)-1-i s o p r o p y l-4-$} methylcyclohexane (22d)

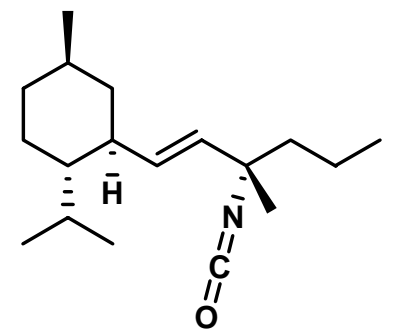

Prepared as per 6d from allylcarbamate 21d. Gave a light yellow oil as the pure $(S)$ allylisocyanate $\mathbf{2 2 d}$ (53 mg, 99\%). 99\% de (measured by gas chromatography). ${ }^{\mathbf{1}} \mathbf{H}$ NMR $\left(300 \mathrm{MHz}, \mathrm{CDCl}_{3}\right) \delta(\mathrm{ppm}) 5.42\left(\mathrm{dd}, 1 \mathrm{H}, J_{1}=14.9 \mathrm{~Hz}, J_{2}=8.8 \mathrm{~Hz}\right), 5.31(\mathrm{~d}, 1 \mathrm{H}, J=14.9$ $\mathrm{Hz}), 1.94-1.70(\mathrm{~m}, 3 \mathrm{H}), 1.64-1.52(\mathrm{~m}, 4 \mathrm{H}), 1.50-1.25$ (m, 4H), 1.36 (s, 3H), 1.02-0.80 (m, $6 \mathrm{H}), 0.88(\mathrm{~d}, 3 \mathrm{H}, J=6.6 \mathrm{~Hz}), 0.87(\mathrm{~d}, 3 \mathrm{H}, J=6.6 \mathrm{~Hz}), 0.69(\mathrm{~d}, 3 \mathrm{H}, J=6.6 \mathrm{~Hz}) .{ }^{13} \mathrm{C}$ NMR $\left(75.5 \mathrm{MHz}, \mathrm{CDCl}_{3}\right) \delta(\mathrm{ppm}) 133.6(\mathrm{~d}), 133.2(\mathrm{~d}), 109.2(\mathrm{~s}), 61.7(\mathrm{~s}), 47.1(\mathrm{~d}), 45.8(\mathrm{t}), 44.3$ $(\mathrm{d}), 43.2(\mathrm{t}), 35.1(\mathrm{t}), 32.4(\mathrm{~d}), 29.6(\mathrm{q}), 28.2(\mathrm{~d}), 24.0(\mathrm{t}), 22.5(\mathrm{q}), 21.4(\mathrm{q}), 17.7(\mathrm{t}), 15.1$ (q), 14.1 (q). IR (neat, $\left.\mathrm{cm}^{-1}\right)$ 2913, 2872, 2260, 1456. LRMS ( $\mathrm{m} / \mathrm{z}$, relative intensity) 277 $\left(\mathrm{M}^{+}, 3\right), 234\left((\mathrm{M}-\mathrm{HNCO})^{+}, 44\right), 191$ (31), 96 (100). HRMS calcd for $\mathrm{C}_{18} \mathrm{H}_{31} \mathrm{NO}$ : 277.2405, found: $277.2399 .[\alpha]_{\mathrm{D}}{ }^{20}=-52.4\left(c=1.52, \mathrm{CHCl}_{3}\right)$. 


\section{1-((S,E)-2-Isocyanato-4-((1S,2S,5R)-2-isopropyl-5-methylcyclohexyl)but-3-en-2-} yl)benzene (6e)

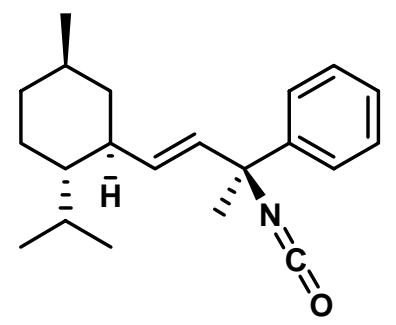

Prepared as per 6d from allylcarbamate 11e. The resulting light yellow oil was purified by flash chromatography (100\% hexanes) to yield pure (S)-allylisocyanate 6 e $(217 \mathrm{mg}, 80 \%)$ as a colorless oil. 94\%de (measured by gas chromatography). ${ }^{1} \mathbf{H}$ NMR (300 MHz, $\left.\mathrm{CDCl}_{3}\right) \delta(\mathrm{ppm}) 7.42-7.24(\mathrm{~m}, 5 \mathrm{H}), 5.67(\mathrm{~d}, 1 \mathrm{H}, J=15.4 \mathrm{~Hz}), 5.53\left(\mathrm{dd}, 1 \mathrm{H}, J_{1}=15.4 \mathrm{~Hz}\right.$, $\left.J_{2}=9.1 \mathrm{~Hz}\right), 2.04-1.78(\mathrm{~m}, 3 \mathrm{H}), 1.75(\mathrm{~s}, 3 \mathrm{H}), 1.64-1.53(\mathrm{~m}, 2 \mathrm{H}), 1.43-1.25(\mathrm{~m}, 1 \mathrm{H}), 1.08-$ $0.82(\mathrm{~m}, 4 \mathrm{H}), 0.90(\mathrm{~d}, 3 \mathrm{H}, J=7.2 \mathrm{~Hz}), 0.87$ (d, $3 \mathrm{H}, J=6.6 \mathrm{~Hz}), 0.71$ (d, $3 \mathrm{H}, J=6.6 \mathrm{~Hz})$. ${ }^{13} \mathrm{C}$ NMR (75.5 MHz, $\left.\mathrm{CDCl}_{3}\right) \delta(\mathrm{ppm}) 144.7$ (s), 134.3 (d), 133.9 (d), 128.4 (d), 127.2 (d), 125.2 (d), 116.5 (s), 63.8 (s), 47.2 (d), 44.3 (d), 43.0 (t), 35.1 (t), 32.4 (d), 30.9 (q), 28.4 (d), 24.1 (t), 22.5 (q), 21.4 (q), 15.3 (q). IR (neat, $\mathrm{cm}^{-1}$ ) 3062, 3029, 2954, 2917, 2846, 2253, 1451, 974. LRMS $\left(\mathrm{m} / \mathrm{z}\right.$, relative intensity) $311\left(\mathrm{M}^{+}, 10\right), 296\left(\left(\mathrm{M}-\mathrm{CH}_{3}\right)^{+}, 10\right), 268((\mathrm{M}-$ $\left.\mathrm{HNCO}^{+}, 12\right), 158(70), 146(100), 138$ (70), 83 (70). HRMS calcd for $\mathrm{C}_{21} \mathrm{H}_{29} \mathrm{NO}$ : 311.2249, found: $311.2251 .[\alpha]_{\mathrm{D}}{ }^{20}=-84.8\left(c=1.51, \mathrm{CHCl}_{3}\right)$.

1-((R,E)-2-Isocyanato-4-((1S,2S,5R)-2-isopropyl-5-methylcyclohexyl)but-3-en-2yl)benzene (22e)

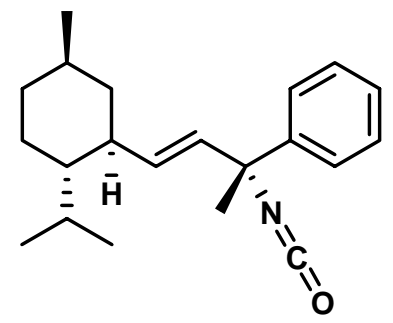

Prepared as per 6d from allylcarbamate 21e. The resulting light yellow oil was purified by flash chromatography (100\% hexanes) to yield pure $(R)$-allylisocyanate $22 \mathbf{e}(64 \mathrm{mg}, 79 \%)$ as a colorless oil. 87\%de (measured by gas chromatography). $\mathbf{1}_{\mathbf{H}} \mathbf{~ N M R}(300 \mathrm{MHz}$, $\left.\mathrm{CDCl}_{3}\right) \delta(\mathrm{ppm}) 7.42-7.24(\mathrm{~m}, 5 \mathrm{H}), 5.69(\mathrm{~d}, 1 \mathrm{H}, J=15.4 \mathrm{~Hz}), 5.53\left(\mathrm{dd}, 1 \mathrm{H}, J_{1}=15.1 \mathrm{~Hz}\right.$, $\left.J_{2}=9.1 \mathrm{~Hz}\right), 2.01-1.84(\mathrm{~m}, 1 \mathrm{H}), 1.83-1.57(\mathrm{~m}, 5 \mathrm{H}), 1.75(\mathrm{~s}, 3 \mathrm{H}), 1.44-1.26(\mathrm{~m}, 1 \mathrm{H}), 1.04-$ $0.84(\mathrm{~m}, 3 \mathrm{H}), 0.88(\mathrm{~d}, 6 \mathrm{H}, J=7.2 \mathrm{~Hz}), 0.70(\mathrm{~d}, 3 \mathrm{H}, J=6.6 \mathrm{~Hz}) .{ }^{13} \mathrm{C}$ NMR $(75.5 \mathrm{MHz}$, $\left.\mathrm{CDCl}_{3}\right) \delta(\mathrm{ppm}) 144.7$ (s), 134.3 (d), 133.9 (d), 128.4 (d), 127.2 (d), 125.2 (d), 116.5 (s), $63.8(\mathrm{~s}), 47.2(\mathrm{~d}), 44.3$ (d), $43.0(\mathrm{t}), 35.1(\mathrm{t}), 32.4$ (d), 30.8 (q), 28.3 (d), 24.1 (t), 22.5 (q), 21.4 (q), 15.3 (q). IR (neat, $\mathrm{cm}^{-1}$ ) 2955, 2920, 2872, 2249, 1450. LRMS ( $\mathrm{m} / \mathrm{z}$, relative intensity) $311\left(\mathrm{M}^{+}, 13\right), 296\left(\left(\mathrm{M}-\mathrm{CH}_{3}\right)^{+}, 10\right), 268\left(\mathrm{M}^{+}-\mathrm{HNCO}, 16\right), 158$ (61), 146 (100), 138 (75), $95(68), 83(67)$. HRMS calcd for $\mathrm{C}_{21} \mathrm{H}_{29} \mathrm{NO}: 311.2249$, found: 311.2251 . [ $\left.\alpha\right]_{\mathrm{D}}{ }^{20}$ $=-52.9\left(c=1.16, \mathrm{CHCl}_{3}\right)$. 
1-((R,E)-2-Isocyanato-4-((1S,2S,5R)-2-isopropyl-5-methylcyclohexyl)-2-methylbut-3enyl)benzene (6f)

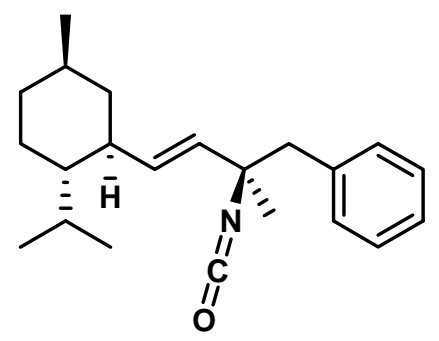

Prepared as per 6d from allylcarbamate 11f. Gave a light yellow oil as the pure $(R)$ allylisocyanate $\mathbf{6 f}(40 \mathrm{mg},>99 \%) .99 \%$ de (measured by gas chromatography). ${ }^{\mathbf{1}} \mathbf{H}$ NMR $\left(300 \mathrm{MHz}, \mathrm{CDCl}_{3}\right) \delta(\mathrm{ppm}) 7.33-7.17(\mathrm{~m}, 5 \mathrm{H}), 5.43(\mathrm{~d}, 1 \mathrm{H}, J=15.4 \mathrm{~Hz}), 5.35\left(\mathrm{dd}, 1 \mathrm{H}, J_{1}\right.$ $\left.=15.4 \mathrm{~Hz}, J_{2}=8.3\right), 2.83(\mathrm{~s}, 2 \mathrm{H}), 1.95-1.84(\mathrm{~m}, 1 \mathrm{H}), 1.74-1.68(\mathrm{~m}, 2 \mathrm{H}), 1.63-1.51(\mathrm{~m}$, $2 \mathrm{H}), 1.40(\mathrm{~s}, 3 \mathrm{H}), 1.38-1.23(\mathrm{~m}, 1 \mathrm{H}), 1.18-0.75(\mathrm{~m}, 4 \mathrm{H}), 0.87(\mathrm{~d}, 3 \mathrm{H}, J=6.6 \mathrm{~Hz}), 0.86(\mathrm{~d}$, $3 \mathrm{H}, J=6.6 \mathrm{~Hz}), 0.71(\mathrm{~d}, 3 \mathrm{H}, J=6.6 \mathrm{~Hz}) .{ }^{13} \mathrm{C}$ NMR $\left(75.5 \mathrm{MHz}, \mathrm{CDCl}_{3}\right) \delta(\mathrm{ppm}) 136.1$ (s), 134.2 (d), 132.8 (d), 130.7 (d), 127.9 (d), 126.8 (d), 123.2 (s), 61.5 (s), 49.6 (t), 47.0 (d), 44.1 (d), 43.0 (t), 35.1 (t), 32.4 (d), 28.8 (q), 28.1 (d), $24.1(\mathrm{t}), 22.5$ (q), 21.4 (q), 15.3 (q). IR (neat, $\left.\mathrm{cm}^{-1}\right) 3029,2954,2921,2870,2257$. LRMS ( $\mathrm{m} / \mathrm{z}$, relative intensity) 325 $\left(\mathrm{M}^{+}, 1\right), 234\left(\left(\mathrm{M}-\mathrm{C}_{7} \mathrm{H}_{7}\right)^{+}, 43\right), 310\left(\left(\mathrm{M}-\mathrm{CH}_{3}\right)^{+}, 2\right), 191$ (20), 96 (100). HRMS calcd for $\mathrm{C}_{22} \mathrm{H}_{31} \mathrm{NO}: 325.2405$, found: $325.2402 .[\alpha]_{\mathrm{D}}{ }^{20}=-29.1\left(c=1.01, \mathrm{CHCl}_{3}\right)$.

\section{1-((S,E)-2-Isocyanato-4-((1S,2S,5R)-2-isopropyl-5-methylcyclohexyl)-2-methylbut-3- enyl)benzene (22f)}

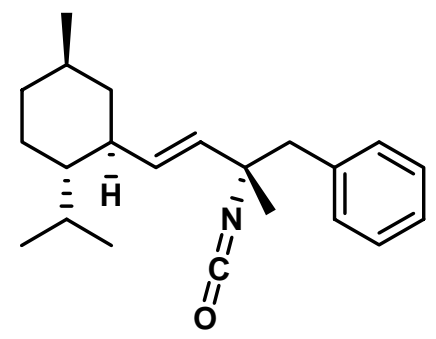

Prepared as per 6d from allylcarbamate 21f. Gave a light yellow oil as the pure (S)allylisocyanate $22 \mathrm{f}(65 \mathrm{mg},>99 \%) .99 \%$ de (measured by gas chromatography). ${ }^{1} \mathbf{H}$ NMR $\left(300 \mathrm{MHz}, \mathrm{CDCl}_{3}\right) \delta(\mathrm{ppm})$ 7.32-7.25 (m, 3H), 7.19-7.17 (m, 2H) 5.43-5.30 (m, 2H), 2.84 (s, $2 \mathrm{H}), 1.94-1.83(\mathrm{~m}, 1 \mathrm{H}), 1.73-1.71(\mathrm{~m}, 1 \mathrm{H}), 1.69-1.50(\mathrm{~m}, 3 \mathrm{H}), 1.40(\mathrm{~s}, 3 \mathrm{H}), 1.37-1.25$ $(\mathrm{m}, 1 \mathrm{H}), 1.18-0.74(\mathrm{~m}, 4 \mathrm{H}), 0.86(\mathrm{~d}, 3 \mathrm{H}, J=6.6 \mathrm{~Hz}), 0.82(\mathrm{~d}, 3 \mathrm{H}, J=7.1 \mathrm{~Hz}), 0.69(\mathrm{~d}, 3 \mathrm{H}$, $J=7.1 \mathrm{~Hz}) .{ }^{13} \mathrm{C}$ NMR $\left(75.5 \mathrm{MHz}, \mathrm{CDCl}_{3}\right) \delta(\mathrm{ppm}) 136.2(\mathrm{~s}), 134.2$ (d), 132.7 (d), 130.6 (d), 127.9 (d), 126.8 (d), $123.2(\mathrm{~s}), 61.9(\mathrm{~s}), 49.5(\mathrm{t}), 47.0$ (d), 44.2 (d), $43.2(\mathrm{t}), 35.1(\mathrm{t})$, 32.4 (d), 29.4 (q), 28.0 (d), 24.0 (t), 22.5 (q), 21.4 (q), 15.3 (q). IR (neat, $\mathrm{cm}^{-1}$ ) 2954, 2923, 2870, 2257, 1454, 701. LRMS $\left(\mathrm{m} / \mathrm{z}\right.$, relative intensity, CI: $\left.\mathrm{NH}_{3}\right) 343\left(\mathrm{MNH}_{4}{ }^{+}, 2\right), 326$ $\left(\mathrm{MH}^{+}, 1\right), 283(100), 234\left(\left(\mathrm{M}-\mathrm{C}_{7} \mathrm{H}_{7}\right)^{+}, 83\right), 191$ (86), 96 (77). HRMS calcd for $\mathrm{C}_{22} \mathrm{H}_{32} \mathrm{NO}$ : 326.2484, found: $326.2478 .[\alpha]_{\mathrm{D}}{ }^{20}=-46.2\left(c=1.13, \mathrm{CHCl}_{3}\right)$. 
Formation of Fmoc-protected allylic amines

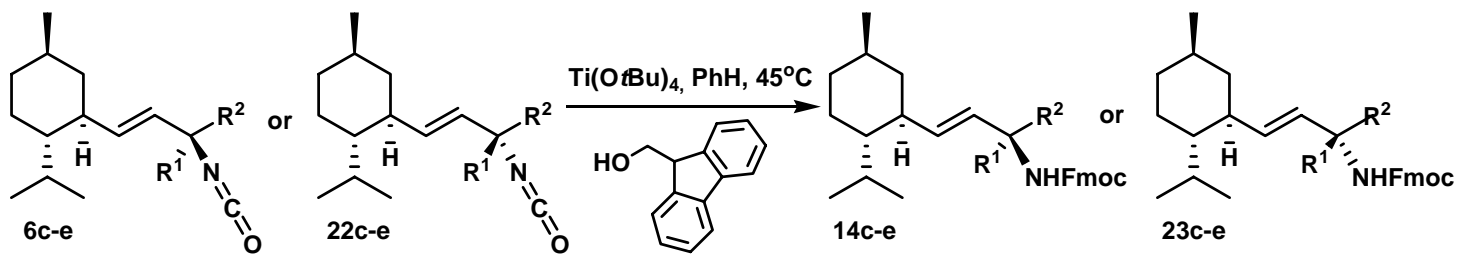

(9H-Fluoren-9-yl)methyl-(R,E)-1-((1S,2S,5R)-2-isopropyl-5-methylcyclohexyl)-3methylhex-1-en-3-ylcarbamate (14d)

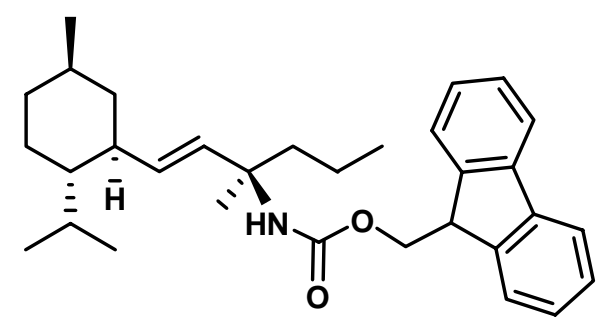

To a solution of allylisocyanate $\mathbf{6 d}(900 \mathrm{mg}, 3.24 \mathrm{mmol}$ ) and 9-fluorenemethanol (954 mg, $4.86 \mathrm{mmol})$ in freshly distilled benzene was added titanium(IV) tert-butoxide $(124 \mu \mathrm{L}, 0.32$ mmol). The solution was heated to $45^{\circ} \mathrm{C}$ for $3 \mathrm{~h}$. The reaction mixture was then poured over a saturated aqueous solution of ammonium chloride and it was extracted three times with diethyl ether $(20 \mathrm{~mL})$. The organic layers were combined, washed with brine, dried with $\mathrm{MgSO}_{4}$, filtered and concentrated under reduced pressure. The resulting oil was purified by flash chromatography (10\% diethyl ether in hexanes) to yield pure $(R)$ allylcarbamate $\mathbf{1 4 d}\left(1.45 \mathrm{~g}, 95 \%\right.$, colorless oil). ${ }^{\mathbf{1}} \mathbf{H}$ NMR $\left(300 \mathrm{MHz}, \mathrm{CDCl}_{3}\right) 7.77$ (d, $2 \mathrm{H}$, $J=7.2 \mathrm{~Hz}$ ), 7.60 (d, 2H, $J=7.2 \mathrm{~Hz}), 7.40$ (t, $2 \mathrm{H}, J=7.2 \mathrm{~Hz}$ ), 7.32 (t, 2H, $J=7.2 \mathrm{~Hz}$ ), 5.49 (br d, $1 \mathrm{H}, J=16.0 \mathrm{~Hz}$ ), $5.24\left(\mathrm{br} \mathrm{dd}, 1 \mathrm{H}, J_{1}=16 \mathrm{~Hz}, J_{2}=8.8 \mathrm{~Hz}\right.$ ), $4.71(\mathrm{br} \mathrm{s}, 1 \mathrm{H}), 4.36$ (br s, $2 \mathrm{H}), 4.22(\mathrm{t}, 1 \mathrm{H}, J=6.6 \mathrm{~Hz}), 1.86-1.59(\mathrm{~m}, 6 \mathrm{H}), 1.38-1.19(\mathrm{~m}, 6 \mathrm{H}), 1.02-0.82(\mathrm{~m}, 8 \mathrm{H})$, $0.862(\mathrm{~d}, 3 \mathrm{H}, J=6.6 \mathrm{~Hz}), 0.856(\mathrm{~d}, 3 \mathrm{H}, J=6.6 \mathrm{~Hz}), 0.69(\mathrm{~d}, 3 \mathrm{H}, J=7.1 \mathrm{~Hz}) .{ }^{13} \mathbf{C} \mathbf{N M R}$ $\left(75.5 \mathrm{MHz}, \mathrm{CDCl}_{3}\right) \delta(\mathrm{ppm}) 154.5$ (s), $144.2(\mathrm{~s}), 141.4(\mathrm{~s}), 134.3(\mathrm{~d}), 133.3$ (d), $127.6(\mathrm{~d})$, 127.0 (d), 125.1 (d), 120.0 (d), 65.9 (t), 56.0 (s), 47.5 (d), 47.2 (d), 44.8 (d), 43.3 (t), 42.5 (t), $35.3(\mathrm{t}), 32.5$ (d), $28.2(\mathrm{~d}), 24.9(\mathrm{q}), 24.1(\mathrm{t}), 22.7$ (q), $21.5(\mathrm{q}), 17.3(\mathrm{t}), 15.3(\mathrm{q}), 14.5$ (q). IR (neat, $\mathrm{cm}^{-1}$ ) 3435, 3351, 2955, 2927, 2869, 1730, 1499, 1450. LRMS $(\mathrm{m} / \mathrm{z}$, relative intensity) $473\left(\mathrm{M}^{+}, 5\right), 430\left(\left(\mathrm{M}-\mathrm{C}_{3} \mathrm{H}_{7}\right)^{+}, 32\right), 208$ (48), 178 (100). HRMS calcd for $\mathrm{C}_{32} \mathrm{H}_{43} \mathrm{NO}_{2}$ : 473.3294, found: $473.3284 .[\alpha]_{\mathrm{D}}^{20}=-42.9\left(c=1.13, \mathrm{CHCl}_{3}\right)$. 
(9H-Fluoren-9-yl)methyl-(S,E)-1-((1S,2S,5R)-2-isopropyl-5-methylcyclohexyl)-3methylhex-1-en-3-ylcarbamate (23d)<smiles>CCC[C@](C)(/C=C/[C@H]1C[C@H](C)CC[C@H]1C(C)C)NC(=O)OCC1c2ccccc2-c2ccccc21</smiles>

Prepared as per 14d from allylisocyanate 22d. The resulting oil was purified by flash chromatography ( $10 \%$ of diethyl ether in hexanes) to yield pure (S)-allylcarbamate 23d (216 mg, 84\%, colorless oil) ${ }^{1} \mathbf{H}$ NMR (300 MHz, $\left.\mathrm{CDCl}_{3}\right) 7.77$ (d, 2H, $J=7.7 \mathrm{~Hz}$ ), 7.60 $(\mathrm{d}, 2 \mathrm{H}, J=7.2 \mathrm{~Hz}), 7.40$ (t, $2 \mathrm{H}, J=7.4 \mathrm{~Hz}), 7.32(\mathrm{t}, 2 \mathrm{H}, J=7.2 \mathrm{~Hz}), 5.47$ (br d, $1 \mathrm{H}, J=$ $15.4 \mathrm{~Hz}$ ), $5.24\left(\mathrm{br} \mathrm{dd}, 1 \mathrm{H}, J_{1}=15.4 \mathrm{~Hz}, J_{2}=9.4 \mathrm{~Hz}\right), 4.71(\mathrm{br} \mathrm{s}, 1 \mathrm{H}), 4.35(\mathrm{br} \mathrm{s}, 2 \mathrm{H}), 4.22$ $(\mathrm{t}, 1 \mathrm{H}, J=6.6 \mathrm{~Hz}), 1.83-1.55(\mathrm{~m}, 8 \mathrm{H}), 1.43-1.19(\mathrm{~m}, 7 \mathrm{H}), 1.01-0.78(\mathrm{~m}, 5 \mathrm{H}), 0.86(\mathrm{~d}, 3 \mathrm{H}, J$ $=6.1 \mathrm{~Hz}), 0.85(\mathrm{~d}, 3 \mathrm{H}, J=7.2 \mathrm{~Hz}), 0.67(\mathrm{~d}, 3 \mathrm{H}, J=6.6 \mathrm{~Hz}) . \quad$ IR (neat, $\left.\mathrm{cm}^{-1}\right) 3443,3351$, 2954, 2925, 2871, 1731, 1499, 1451, 1235, 1099. LRMS ( $\mathrm{m} / \mathrm{z}$, relative intensity) $473\left(\mathrm{M}^{+}\right.$, 3), $430\left(\left(\mathrm{M}-\mathrm{C}_{3} \mathrm{H}_{7}\right)^{+}, 23\right), 208(60), 178(100)$. HRMS calcd for $\mathrm{C}_{32} \mathrm{H}_{43} \mathrm{NO}_{2}$ : 473.3294, found: $473.3303 .[\alpha]_{D}{ }^{20}=-33.4\left(c=1.08, \mathrm{CHCl}_{3}\right)$.

(9H-Fluoren-9-yl)methyl-(S,E)-4-((1S,2S,5R)-2-isopropyl-5-methylcyclohexyl)-2phenylbut-3-en-2-ylcarbamate (14e)

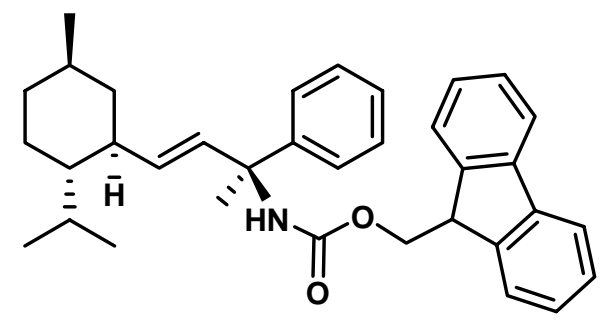

Prepared as per 14d from allylisocyanate 6e. The resulting oil was purified by flash chromatography (10\% of diethyl ether in hexanes) to yield pure (S)-allylcarbamate 14e (161 mg, 86\%, colorless oil). ${ }^{1} \mathbf{H}$ NMR $\left(300 \mathrm{MHz}, \mathrm{CDCl}_{3}\right) 7.79$ (br d, $2 \mathrm{H}, J=6.6 \mathrm{~Hz}$ ), 7.63 (d (br), $2 \mathrm{H}, J=6.6 \mathrm{~Hz}$ ), 7.45-7.24 (m, 9H), 5.94 (br d, $1 \mathrm{H}, J=15.4 \mathrm{~Hz}$ ), 5.37 (dd, $1 \mathrm{H}$, $\left.J_{1}=15.9 \mathrm{~Hz}, J_{2}=9.4 \mathrm{~Hz}\right), 5.21(\mathrm{~s}, 1 \mathrm{H}), 4.41(\mathrm{~d}, 2 \mathrm{H}, J=7.2 \mathrm{~Hz}), 4.23(\mathrm{br} \mathrm{s}, 1 \mathrm{H}), 2.00-1.98$ (m, 1H), 1.88-1.84 (m, 1H), 1.78-1.62 (m, 3H), $1.74(\mathrm{~s}, 3 \mathrm{H}), 1.47-1.34(\mathrm{~m}, 1 \mathrm{H}), 1.07-0.83$ (m, 4H), $0.91(\mathrm{~d}, 3 \mathrm{H}, J=7.2 \mathrm{~Hz}), 0.90(\mathrm{~d}, 3 \mathrm{H}, J=6.1 \mathrm{~Hz}), 0.76(\mathrm{~d}, 3 \mathrm{H}, J=6.6 \mathrm{~Hz}) . \quad$ IR (neat, $\mathrm{cm}^{-1}$ ) 2950, 2921, 2871, 1725, 1708, 1496, 1447, 1244. LRMS $(\mathrm{m} / \mathrm{z}$, relative intensity) $507\left(\mathrm{M}^{+}, 4\right), 178(100), 146$ (78). HRMS calcd for $\mathrm{C}_{35} \mathrm{H}_{41} \mathrm{NO}_{2}: 507.3137$, found: 507.3142. $[\alpha]_{\mathrm{D}}^{20}=-44.5\left(c=1.14, \mathrm{CHCl}_{3}\right)$. 
(9H-Fluoren-9-yl)methyl-(R,E)-4-((1S,2S,5R)-2-isopropyl-5-methylcyclohexyl)-2phenylbut-3-en-2-ylcarbamate (23e)<smiles>CC1CCC(C(C)C)[C@H](/C=C/[C@H](C)c2ccccc2)C1</smiles>

Prepared as per 14d from allylisocyanate 22e. The resulting oil was purified by flash chromatography ( $10 \%$ of diethyl ether in hexanes) to yield pure $(R)$-allylcarbamate 23e (70 mg, 87\%, white solid). m.p.: $55-56{ }^{\circ} \mathrm{C} \mathbf{1}_{\mathbf{H}} \mathbf{N M R}\left(300 \mathrm{MHz}, \mathrm{CDCl}_{3}\right) 7.76$ (br d, $2 \mathrm{H}, J=$ $7.2 \mathrm{~Hz}$ ), 7.59 (br m, 2H), 7.43-7.22 (m, 9H), 5.87 (br d, 1H, $J=15.4 \mathrm{~Hz}), 5.31$ (dd, 1H, $J_{1}=$ $\left.15.4 \mathrm{~Hz}, J_{2}=9.4 \mathrm{~Hz}\right), 5.17(\mathrm{~s}, 1 \mathrm{H}), 4.36(\mathrm{~d}, 2 \mathrm{H}, J=6.1 \mathrm{~Hz}), 4.20(\mathrm{br} \mathrm{s}, 1 \mathrm{H}), 1.97-1.94(\mathrm{~m}$, $1 \mathrm{H}), 1.82-1.56(\mathrm{~m}, 7 \mathrm{H}), 1.44-1.30(\mathrm{~m}, 1 \mathrm{H}), 1.03-0.92(\mathrm{~m}, 4 \mathrm{H}), 0.88(\mathrm{~d}, 3 \mathrm{H}, J=6.6 \mathrm{~Hz})$, $0.86(\mathrm{~d}, 3 \mathrm{H}, J=6.6 \mathrm{~Hz}), 0.68(\mathrm{~d}, 3 \mathrm{H}, J=7.1 \mathrm{~Hz}) . \quad$ IR $\left(\mathrm{CHCl}_{3}, \mathrm{~cm}^{-1}\right) 3064,3021,2952$, 2918, 2870, 1727, 1495, 1447, 1244. LRMS $\left(\mathrm{m} / \mathrm{z}\right.$, relative intensity) $507\left(\mathrm{M}^{+}, 3\right), 179$ (100), 165 (78), $146(83)$. HRMS calcd for $\mathrm{C}_{35} \mathrm{H}_{41} \mathrm{NO}_{2}$ : 507.3137, found: 507.3127. [ $\left.\alpha\right]_{\mathrm{D}}{ }^{20}$ $=-34.2\left(c=0.99, \mathrm{CHCl}_{3}\right)$.

(9H-Fluoren-9-yl)methyl-(R,E)-3-((1S,2S,5R)-2-isopropyl-5-methylcyclohexyl)-1(trimethylsilyl)allylcarbamate (14c)

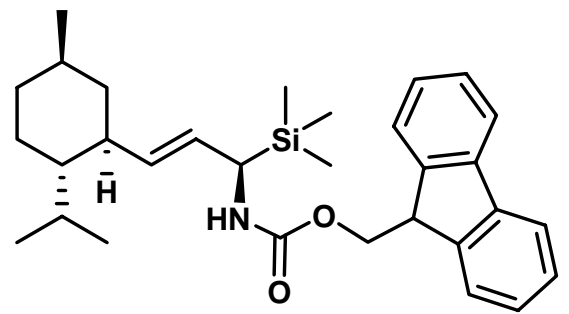

Prepared as per 14d from allylisocyanate 6c. The resulting oil was purified by flash chromatography ( $10 \%$ of diethyl ether in hexanes) to yield pure $(R)$-allylcarbamate $14 \mathbf{c}$ (283 mg, 70\%, colorless oil). ${ }^{1} \mathbf{H}$ NMR (300 MHz, CDCl 3 ) 7.78 (d, $2 \mathrm{H}, J=7.2 \mathrm{~Hz}$ ), 7.62 $(\mathrm{d}, 2 \mathrm{H}, J=7.7 \mathrm{~Hz}), 7.41(\mathrm{t}, 2 \mathrm{H}, J=7.4 \mathrm{~Hz}), 7.33(\mathrm{t}, 2 \mathrm{H}, J=7.2 \mathrm{~Hz}), 5.39$ (dd, $1 \mathrm{H}, J_{1}=$ $\left.15.4 \mathrm{~Hz}, J_{2}=6.6 \mathrm{~Hz}\right), 5.17$ (ddd, $1 \mathrm{H}, J_{1}=15.4 \mathrm{~Hz}, J_{2}=8.8 \mathrm{~Hz}, J_{3}=1.1 \mathrm{~Hz}$ ), $4.72($ br d, $1 \mathrm{H}$, $J=8.8 \mathrm{~Hz}$ ), $4.42(\mathrm{~d}, 2 \mathrm{H}, J=7.2 \mathrm{~Hz}), 4.26(\mathrm{t}, 1 \mathrm{H}, J=6.9 \mathrm{~Hz}), 3.79(\mathrm{br} \mathrm{t}, 1 \mathrm{H}, J=7.2 \mathrm{~Hz})$, 1.96-1.82 (m, 2H), 1.80-1.70 (m, 1H), 1.62-1.58 (m, 2H), 1.45-1.28 (m, 1H), 1.06-0.80 (m, $4 \mathrm{H}), 0.88(\mathrm{~d}, 3 \mathrm{H}, J=6.6 \mathrm{~Hz}), 0.83(\mathrm{~d}, 3 \mathrm{H}, J=7.2 \mathrm{~Hz}), 0.69(\mathrm{~d}, 3 \mathrm{H}, J=6.6 \mathrm{~Hz}), 0.07(\mathrm{~s}$, 9H). ${ }^{13} \mathrm{C}$ NMR $\left(75.5 \mathrm{MHz}, \mathrm{CDCl}_{3}\right) \delta(\mathrm{ppm}) 156.3$ (s), 144.1 (s), 141.3 (s), 133.0 (d), 127.6 (d), 127.0 (d), 125.1 (d), 120.0 (d), 66.6 (t), 47.4 (d), 47.2 (d), 45.1 (d), 44.7 (d), 43.7 $(\mathrm{t}), 35.2(\mathrm{t}), 32.6(\mathrm{~d}), 28.0(\mathrm{~d}), 24.1(\mathrm{t}), 22.7(\mathrm{q}), 21.4(\mathrm{q}), 15.3(\mathrm{q}),-3.6(\mathrm{q})$. IR (neat, $\left.\mathrm{cm}^{-1}\right)$ 2952, 2917, 2871, 1700. LRMS ( $\mathrm{m} / \mathrm{z}$, relative intensity) $489\left(\mathrm{M}^{+}, 2\right), 266$ (82), 178 (100), 172 (86), 128 (85). HRMS calcd for $\mathrm{C}_{31} \mathrm{H}_{43} \mathrm{NO}_{2} \mathrm{Si}: 489.3063$, found: 489.3073. [ $\left.\alpha\right]_{\mathrm{D}}{ }^{20}=-$ $9.9\left(c=1.47, \mathrm{CHCl}_{3}\right)$. 
(9H-Fluoren-9-yl)methyl-(S,E)-3-((1S,2S,5R)-2-isopropyl-5-methylcyclohexyl)-1(trimethylsilyl)allylcarbamate (23c)<smiles>CC1CCC(C(C)C)C(/C=C/[C@H](NC(=O)OCC2c3ccccc3-c3ccccc32)[Si](C)(C)C)C1</smiles>

Prepared as per 14d from allylisocyanate 22c. The resulting oil was purified by flash chromatography (10\% of diethyl ether in hexanes) to yield pure (S)-allylcarbamate 23c (106 mg, 90\%, white solid). m.p.: $43-44{ }^{\circ} \mathrm{C} \quad \mathbf{1}_{\mathbf{H}} \mathbf{~ N M R}\left(300 \mathrm{MHz}, \mathrm{CDCl}_{3}\right) 7.77$ (d, 2H, $J$ $=7.2 \mathrm{~Hz}), 7.61(\mathrm{~d}, 2 \mathrm{H}, J=7.7 \mathrm{~Hz}), 7.41(\mathrm{t}, 2 \mathrm{H}, J=7.2 \mathrm{~Hz}), 7.32(\mathrm{t}, 2 \mathrm{H}, J=7.4 \mathrm{~Hz}), 5.40$ $\left(\mathrm{dd}, 1 \mathrm{H}, J_{1}=15.4 \mathrm{~Hz}, J_{2}=5.5 \mathrm{~Hz}\right), 5.11\left(\mathrm{dd}, 1 \mathrm{H}, J_{1}=15.4 \mathrm{~Hz}, J_{2}=9.4 \mathrm{~Hz}\right), 4.67(\mathrm{~d}, 1 \mathrm{H}, J$ $=9.4 \mathrm{~Hz}), 4.42(\mathrm{~d}, 2 \mathrm{H}, J=6.6 \mathrm{~Hz}), 4.25(\mathrm{t}, 1 \mathrm{H}, J=7.2 \mathrm{~Hz}), 3.79(\mathrm{br} \mathrm{m}, 1 \mathrm{H}), 1.94-1.82(\mathrm{~m}$, 2H), $1.71(\mathrm{br} \mathrm{d}, 1 \mathrm{H}, J=12.1 \mathrm{~Hz}), 1.60-1.55(\mathrm{~m}, 2 \mathrm{H}), 1.44-1.26(\mathrm{~m}, 1 \mathrm{H}), 1.00-0.75(\mathrm{~m}, 4 \mathrm{H})$, $0.85(\mathrm{~d}, 6 \mathrm{H}, J=6.1 \mathrm{~Hz}), 0.68(\mathrm{~d}, 3 \mathrm{H}, J=6.6 \mathrm{~Hz}), 0.05(\mathrm{~s}, 9 \mathrm{H}) . \quad$ IR (neat, $\left.\mathrm{cm}^{-1}\right) 3322$ (br), 2952, 2916, 1704, 1589, 1440, 1247, 842. LRMS ( $\mathrm{m} / \mathrm{z}$, relative intensity) $489\left(\mathrm{M}^{+}, 1\right), 179$ (76), 178 (100). HRMS calcd for $\mathrm{C}_{31} \mathrm{H}_{43} \mathrm{NO}_{2} \mathrm{Si} 489.3063$, found: 489.3073. $[\alpha]_{\mathrm{D}}^{20}=-66.9$ $\left(c=0.90, \mathrm{CHCl}_{3}\right)$.

\section{Formation of amino alcohols}

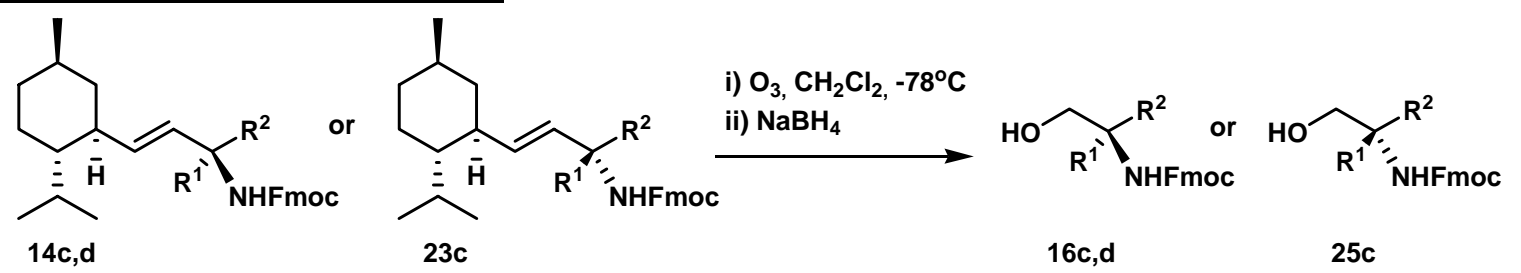

(9H-Fluoren-9-yl)methyl (R)-1-hydroxy-2-methylpentan-2-ylcarbamate (16d)<smiles>CCC[C@H](C)NC(=O)OCC1c2ccccc2-c2ccccc21</smiles>

To a solution of allylcarbamate 14d (181 $\mathrm{mg}, 0.382 \mathrm{mmol})$ and Sudan(III) $(1 \mathrm{mg}$, used as indicator) in dichloromethane $(7.6 \mathrm{~mL}, 0.05 \mathrm{M})$ at $-78{ }^{\circ} \mathrm{C}$, was bubbled ozone until the red color of the reaction mixture turned to a light yellow-brown color. The intermediate ozonide was then treated with sodium borohydride $(289 \mathrm{mg}, 7.64 \mathrm{mmol})$ and the reaction mixture was stirred at $\mathrm{rt}$ for $6 \mathrm{~h}$. A $1 \mathrm{~N} \mathrm{HCl}$ solution was then added dropwise until the $\mathrm{pH}$ of the solution was approximately 6 . The solution was extracted three times with diethyl ether $(10 \mathrm{~mL})$. The organic layers were combined, washed with brine, dried over $\mathrm{MgSO}_{4}$, filtered and concentrated under reduced pressure. The resulting light red oil was purified by flash chromatography (from $20 \%$ of diethyl ether in hexanes to $60 \%$ of diethyl ether in hexanes) to yield pure $(R)$-alcohol 16d (99 mg, 77\%, colorless oil). ${ }^{\mathbf{1}} \mathbf{H} \mathbf{~ N M R}(300 \mathrm{MHz}$, $\left.\mathrm{CDCl}_{3}\right) 7.77(\mathrm{~d}, 2 \mathrm{H}, J=7.2 \mathrm{~Hz}), 7.59(\mathrm{~d}, 2 \mathrm{H}, J=7.2 \mathrm{~Hz}), 7.41(\mathrm{t}, 2 \mathrm{H}, J=7.2 \mathrm{~Hz}), 7.32(\mathrm{t}$, 
$2 \mathrm{H}, J=7.2 \mathrm{~Hz}$ ), 4.79 (br s, 1H), 4.42 (br m, 2H), 4.20 (t, 1H, $J=6.6 \mathrm{~Hz}), 3.59$ (br m, 2H), 1.68-1.55 (m, 1H), 1.55-1.40 (m, 1H), 1.40-1.17 (m, 2H), $1.17(\mathrm{~s}, 3 \mathrm{H}), 0.92(\mathrm{t}, 3 \mathrm{H}, J=7.2$ Hz). ${ }^{13}$ C NMR (75.5 MHz, $\left.\mathrm{CDCl}_{3}\right) \delta(\mathrm{ppm}) 156.1$ (s), 143.8 (s), 141.3 (s), 127.7 (d), 127.0 (d), 124.9 (d), 120.0 (d), $68.9(\mathrm{t}), 66.2(\mathrm{t}), 57.1$ (s), $47.3(\mathrm{~d}), 38.8(\mathrm{t}), 22.3(\mathrm{q}), 16.7$ (t), 14.5 (q). IR (neat, $\mathrm{cm}^{-1}$ ) 3687-3129, 3066, 2958, 2872, 1710, 1695, 1534, 1517, 1449, 1244, 1105. LRMS $\left(\mathrm{m} / \mathrm{z}\right.$, relative intensity) $339\left(\mathrm{M}^{+}, 1\right), 308\left(\left(\mathrm{M}-\mathrm{HOCH}_{2}\right)^{+}, 50\right), 179$ (88), 178 (100), 166 (89), 165 (93), 100 (61). HRMS calcd for $\mathrm{C}_{21} \mathrm{H}_{25} \mathrm{NO}_{3}: 339.1834$, found: $339.1842 .[\alpha]_{\mathrm{D}}^{20}=-1.9\left(c=1.11, \mathrm{CHCl}_{3}\right)$.

(9H-Fluoren-9-yl)methyl (R)-2-hydroxy-1-(trimethylsilyl)ethylcarbamate (16c)

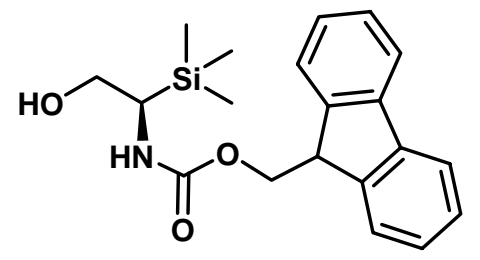

Prepared as per 16d from 14c. The resulting light red oil was purified by flash chromatography (from $20 \%$ of diethyl ether in hexanes to $60 \%$ of diethyl ether in hexanes) to yield pure $(R)$-alcohol 16c (52 $\mathrm{mg}, 83 \%$, colorless oil). e.e. determined by gas chromatography: $96 \%{ }^{\mathbf{1}} \mathbf{H}$ NMR $\left(300 \mathrm{MHz}, \mathrm{CDCl}_{3}\right) 7.77(\mathrm{~d}, 2 \mathrm{H}, J=7.7 \mathrm{~Hz}), 7.59(\mathrm{~d}, 2 \mathrm{H}$, $J=7.2 \mathrm{~Hz}), 7.41(\mathrm{t}, 2 \mathrm{H}, J=7.2 \mathrm{~Hz}), 7.32(\mathrm{t}, 2 \mathrm{H}, J=7.2 \mathrm{~Hz}), 4.82(\mathrm{br} \mathrm{d}, 1 \mathrm{H}, J=8.3 \mathrm{~Hz}$ ), $4.45(\mathrm{~d}, 2 \mathrm{H}, J=7.2 \mathrm{~Hz}), 4.24(\mathrm{t}, 1 \mathrm{H}, J=6.9 \mathrm{~Hz}), 3.82\left(\mathrm{dd}, 1 \mathrm{H}, J_{1}=11.0 \mathrm{~Hz}, J_{2}=2.8 \mathrm{~Hz}\right)$, $3.68\left(\mathrm{dd}, 1 \mathrm{H}, J_{1}=11.6 \mathrm{~Hz}, J_{2}=7.7 \mathrm{~Hz}\right), 3.27\left(\mathrm{td}, 1 \mathrm{H}, J_{1}=8.2 \mathrm{~Hz}, J_{2}=2.8 \mathrm{~Hz}\right), 1.83(\mathrm{br} \mathrm{s}$, 1H), $0.11(\mathrm{~s}, 9 \mathrm{H}) .{ }^{13} \mathrm{C}$ NMR $\left(75.5 \mathrm{MHz}, \mathrm{CDCl}_{3}\right) \delta(\mathrm{ppm}) 158.2(\mathrm{~s}), 143.8(\mathrm{~s}), 141.3(\mathrm{~s})$, 127.7 (d), 127.0 (d), 124.9 (d), 120.0 (d), 66.9 (t), 64.8 (t), 47.3 (d), 45.6 (d), -3.0 (q). IR (neat, $\mathrm{cm}^{-1}$ ) 3579-3146, 3068, 3018, 2952, 2900, 1695, 1251, 842. LRMS $(\mathrm{m} / \mathrm{z}$, relative intensity) $356\left(\mathrm{MH}^{+}, 10\right), 338\left(\left(\mathrm{M}-\mathrm{H}_{2} \mathrm{O}\right)^{+}, 13\right), 179(98), 178$ (100). HRMS calcd for $\mathrm{C}_{20} \mathrm{H}_{26} \mathrm{NO}_{3} \mathrm{Si}: 356.1682$, found: $356.1688 .[\alpha]_{\mathrm{D}}^{20}=16.2\left(c=1.32, \mathrm{CHCl}_{3}\right)$.

(9H-Fluoren-9-yl)methyl (S)-2-hydroxy-1-(trimethylsilyl)ethylcarbamate (25c)

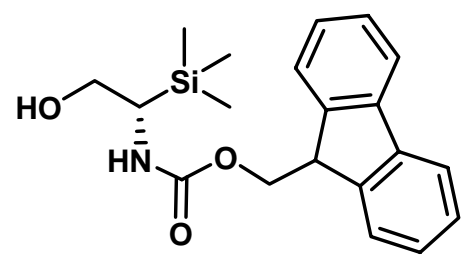

Prepared as per 16d from 23c. The resulting light red oil was purified by flash chromatography (from $20 \%$ of diethyl ether in hexanes to $60 \%$ of diethyl ether in hexanes) to yield pure (S)-alcohol 25c (51 mg, 73\%, colorless oil). e.e. was determined by gas chromatography: 94\%. ${ }^{1} \mathbf{H}$ NMR $\left(300 \mathrm{MHz}, \mathrm{CDCl}_{3}\right) 7.77$ (d, 2H, J = $\left.7.2 \mathrm{~Hz}\right), 7.59$ (d, $2 \mathrm{H}, J=7.2 \mathrm{~Hz}$ ), 7.41 (t, $2 \mathrm{H}, J=7.4 \mathrm{~Hz}$ ), $7.32(\mathrm{t}, 2 \mathrm{H}, J=7.2 \mathrm{~Hz}), 4.86$ (br d, 1H, $J=8.3$ $\mathrm{Hz}), 4.44(\mathrm{~d}, 2 \mathrm{H}, J=7.2 \mathrm{~Hz}), 4.23(\mathrm{t}, 1 \mathrm{H}, J=6.9 \mathrm{~Hz}), 3.82(\mathrm{br} \mathrm{d}, 1 \mathrm{H}, J=9.9 \mathrm{~Hz}), 3.27(\mathrm{br}$ t, $1 \mathrm{H}, J=6.9 \mathrm{~Hz}), 2.14($ br s, $1 \mathrm{H}), 0.11(\mathrm{~s}, 9 \mathrm{H}) .{ }^{13} \mathrm{C}$ NMR $\left(75.5 \mathrm{MHz}, \mathrm{CDCl}_{3}\right) \delta(\mathrm{ppm})$ $158.2(\mathrm{~s}), 143.8$ (s), 141.3 (s), 127.7 (d), 127.0 (d), 124.9 (d), 120.0 (d), 66.9 (t), $64.8(\mathrm{t})$, 47.3 (d), 45.6 (d), -3.0 (q). IR (neat, $\mathrm{cm}^{-1}$ ) 3414, 3332, 3066, 3042, 2952, 2898, 1696, 
1535, 1517, 1250, 842. LRMS $\left(\mathrm{m} / \mathrm{z}\right.$, relative intensity) $355\left(\mathrm{M}^{+}, 1\right), 324\left(\left(\mathrm{M}-\mathrm{CH}_{2} \mathrm{OH}\right)^{+}\right.$, 1), 179 (73), 178 (100), 74 (66). HRMS calcd for $\mathrm{C}_{20} \mathrm{H}_{25} \mathrm{NO}_{3} \mathrm{Si}$ : 355.1604, found: 355.1610. $[\alpha]_{\mathrm{D}}^{20}=-15.8\left(c=1.73, \mathrm{CHCl}_{3}\right)$.

\section{Formation of amino acids}
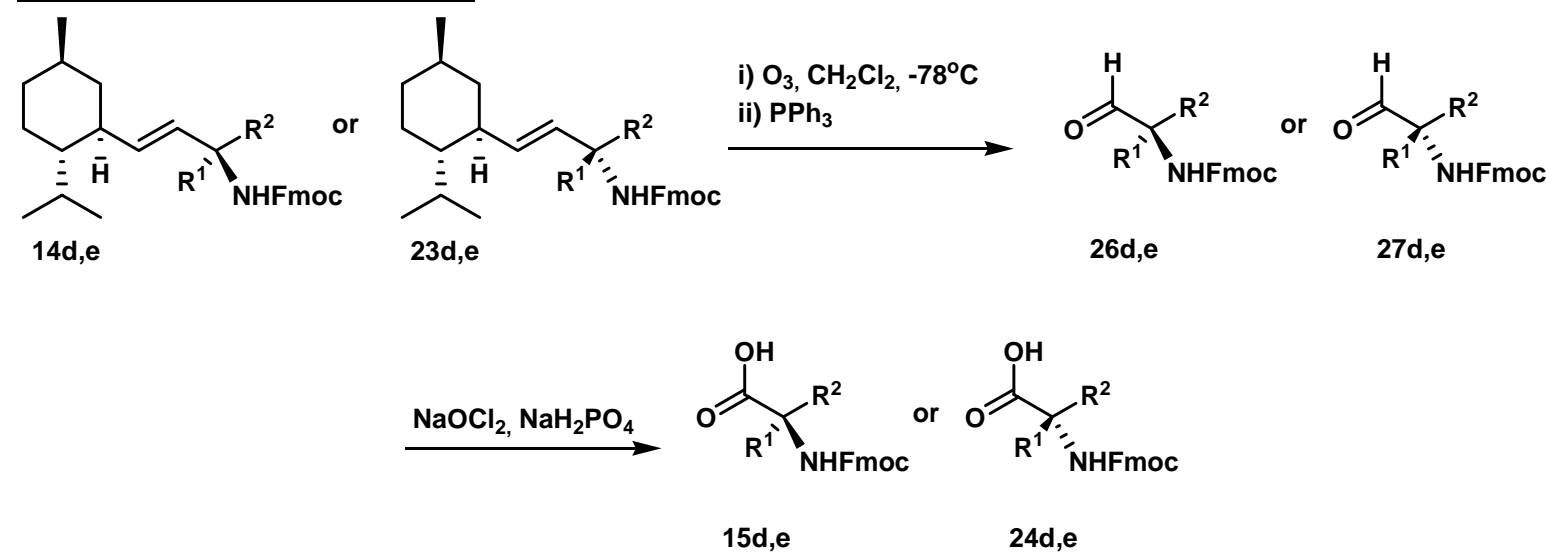

(R)-2-((9H-Fluoren-9-yl)methylcarbamate)-2-methylpentanoic acid (15d)<smiles>CCC[C@H](C)C(=O)NC(=O)OCC1c2ccccc2-c2ccccc21</smiles>

To a solution of allylcarbamate 14d (115 mg, $0.243 \mathrm{mmol})$ and Sudan(III) (1 mg) used as indicator in dichloromethane $(5.0 \mathrm{~mL}, 0.05 \mathrm{M})$ at $-78^{\circ} \mathrm{C}$, was bubbled ozone until the red color of the reaction mixture turned to a yellow-orange color. The intermediate ozonide was then treated with triphenylphosphine $(319 \mathrm{mg}, 1.22 \mathrm{mmol})$ and the reaction mixture stirred at $\mathrm{rt}$ for $15 \mathrm{~h}$. Solvents were then evaporated under reduced pressure. The resulting orange solid was filtered over a short pad of silica gel ( $20 \%$ of diethyl ether in hexanes) to yield (R)-aldehyde 26d (71.4 mg, 87\%, colorless oil). To a solution of this aldehyde (71.4 mg, $0.212 \mathrm{mmol})$ and 2-methyl-2-butene $(0.45 \mathrm{~mL}, 4.2 \mathrm{mmol})$ in $t$-butanol $(0.85 \mathrm{~mL}, 0.25 \mathrm{M})$ was added slowly a solution of sodium chlorite $(38 \mathrm{mg}, 0.42 \mathrm{mmol})$ and sodium hydrogenophosphate $(59 \mathrm{mg}, 0.42 \mathrm{mmol})$ in distilled water $(0.85 \mathrm{~mL}, 0.5 \mathrm{M})$. The reaction mixture was stirred for $15 \mathrm{~h}$. The solution was concentrated under reduced pressure. The residue was diluted in brine and dichloromethane and the solution was extracted three times with dichloromethane $(3 \times 5 \mathrm{~mL})$. The organic layers were combined, washed with brine, dried over $\mathrm{MgSO}_{4}$, filtered and concentrated under reduced pressure. The resulting light yellow solid was purified by flash chromatography ( $5 \%$ of methanol in dichloromethane) to yield pure $(R)$-Fmoc amino acid 15d (74 mg, 86\%, 2 steps, white solid). $>98 \%$ ee (measured by HPLC). m.p.: $73-74{ }^{\circ} \mathrm{C} \mathbf{1}^{\mathbf{1}} \mathbf{H}$ NMR (300 MHz, $\left.\mathrm{CD}_{3} \mathrm{OD}\right) 7.77$ (d, $\left.2 \mathrm{H}, J=7.2 \mathrm{~Hz}\right), 7.64$ $(\mathrm{d}, 2 \mathrm{H}, J=7.2 \mathrm{~Hz}), 7.37$ (t, $2 \mathrm{H}, J=7.4 \mathrm{~Hz}), 7.29$ (t, $2 \mathrm{H}, J=7.4 \mathrm{~Hz}), 4.30$ (d, 2H, $J=6.6$ $\mathrm{Hz}), 4.18(\mathrm{t}, 1 \mathrm{H}, J=6.6 \mathrm{~Hz}), 1.87-1.80(\mathrm{~m}, 2 \mathrm{H}), 1.45(\mathrm{~s}, 3 \mathrm{H}), 1.26-1.21(\mathrm{~m}, 2 \mathrm{H}), 0.89(\mathrm{t}$, $3 \mathrm{H}, J=7.2 \mathrm{~Hz}) .{ }^{13} \mathrm{C}$ NMR $\left(75.5 \mathrm{MHz}, \mathrm{CDCl}_{3}\right) \delta(\mathrm{ppm}) 179.3(\mathrm{~s}), 154.8(\mathrm{~s}), 143.8(\mathrm{~s})$, $141.3(\mathrm{~s}), 127.7$ (d), 127.0 (d), 125.0 (d), 120.0 (d), $66.5(\mathrm{t}), 59.9$ (s), 47.2 (d), 39.0 (t), 23.2 (q), 17.3 (t), 14.0 (q). IR $\left(\mathrm{CHCl}_{3}, \mathrm{~cm}^{-1}\right)$ 3410, 3328 (br), 3066, 3016, 2961, 2935, 2871, 
$1710,1509,1447,1239,1104$. LRMS $\left(\mathrm{m} / \mathrm{z}\right.$, relative intensity) $353\left(\mathrm{M}^{+}, 1\right), 308((\mathrm{M}$ HOCO $\left.)^{+}, 2\right), 196$ (90), 179 (92) 166 (99), 165 (100), 115 (82). HRMS calcd for $\mathrm{C}_{21} \mathrm{H}_{23} \mathrm{NO}_{4}: 353.1627$, found: $353.1633 .[\alpha]_{\mathrm{D}}^{20}=-3.3\left(c=1.33, \mathrm{CHCl}_{3}\right)$.

(S)-2-((9H-Fluoren-9-yl)methylcarbamate)-2-methylpentanoic acid (24d)<smiles>CCCC(C)(NC(=O)OCC1c2ccccc2-c2ccccc21)C(=O)O</smiles>

Prepared as per 15d from 23d. The resulting light yellow solid was purified by flash chromatography (5\% of methanol in dichloromethane) to yield pure (S)-Fmoc amino acid 24d (44 mg, 81\%, 2 steps, white solid). $>98 \%$ ee (measured by HPLC). m.p.: $72-73{ }^{\circ} \mathrm{C} \mathbf{1}^{\mathbf{1}} \mathbf{H}$ NMR $\left(300 \mathrm{MHz}, \mathrm{CD}_{3} \mathrm{OD}\right) 7.77$ (d, 2H, $\left.J=7.2 \mathrm{~Hz}\right), 7.64$ (d, 2H, $\left.J=7.7 \mathrm{~Hz}\right), 7.37$ (t, 2H, $J$ $=7.4 \mathrm{~Hz}), 7.29(\mathrm{t}, 2 \mathrm{H}, J=7.2 \mathrm{~Hz}), 4.30(\mathrm{~d}, 2 \mathrm{H}, J=6.6 \mathrm{~Hz}), 4.19(\mathrm{t}, 1 \mathrm{H}, J=6.6 \mathrm{~Hz}), 1.89-$ $1.74(\mathrm{~m}, 2 \mathrm{H}), 1.46(\mathrm{~s}, 3 \mathrm{H}), 1.32-1.16(\mathrm{~m}, 2 \mathrm{H}), 0.90(\mathrm{t}, 3 \mathrm{H}, J=7.4 \mathrm{~Hz}) .{ }^{13} \mathrm{C}$ NMR $(75.5$ $\left.\mathrm{MHz}, \mathrm{CDCl}_{3}\right) \delta(\mathrm{ppm}) 179.1(\mathrm{~s}), 155.0(\mathrm{~s}), 143.8(\mathrm{~s}), 141.2(\mathrm{~s}), 127.7$ (d), $127.0(\mathrm{~d}), 125.0$ (d), 119.9 (d), 66.5 (t), 59.9 (s), 47.2 (d), 39.1 (t), 23.2 (q), 17.3 (t), 14.0 (q). IR $\left(\mathrm{CHCl}_{3}\right.$, $\left.\mathrm{cm}^{-1}\right)$ 3572-2363, 2957, 2871, 1709, 1447, 1239, 1104. LRMS ( $\mathrm{m} / \mathrm{z}$, relative intensity) 353 $\left(\mathrm{M}^{+}, 1\right), 179$ (78), $178(100), 166(80), 165$ (78). HRMS calcd for $\mathrm{C}_{21} \mathrm{H}_{23} \mathrm{NO}_{4}$ : 353.1627, found: $353.1633 .[\alpha]_{\mathrm{D}}^{20}=1.6\left(c=1.00, \mathrm{CHCl}_{3}\right)$.

(R)-2-((9H-Fluoren-9-yl)methylcarbamate)-2-phenylpropanoic acid (15e)<smiles>C[C@H](NC(=O)OCC1c2ccccc2-c2ccccc21)C(=O)O</smiles>

Prepared as per 15d from 14e. The resulting light yellow solid was purified by flash chromatography (5\% of methanol in dichloromethane) to yield pure $(R)$-Fmoc amino acid 15e (93 mg, 83\%, 2 steps, white solid). 93\%ee (measured by HPLC). m.p.: $98-102{ }^{\circ} \mathrm{C}{ }^{\mathbf{1}} \mathbf{H}$ NMR $\left(300 \mathrm{MHz}, \mathrm{CD}_{3} \mathrm{OD}\right) 7.78(\mathrm{~d}, 2 \mathrm{H}, J=7.2 \mathrm{~Hz}), 7.65-7.64(\mathrm{~m}, 2 \mathrm{H}), 7.50-7.41(\mathrm{~m}, 2 \mathrm{H})$, 7.40-7.22 (m, 7H), $4.30(\mathrm{br} \mathrm{d}, 2 \mathrm{H}, J=5.5 \mathrm{~Hz}), 4.19(\mathrm{~m}, 1 \mathrm{H}), 1.92(\mathrm{~s}, 3 \mathrm{H}) .{ }^{13} \mathrm{C}$ NMR $\left(75.5 \mathrm{MHz}, \mathrm{CDCl}_{3}\right) \delta(\mathrm{ppm}) 176.2(\mathrm{~s}), 154.8(\mathrm{~s}), 143.7(\mathrm{~s}), 141.2(\mathrm{~s}), 128.6(\mathrm{~d}), 127.6(\mathrm{~d})$, 127.0 (d), 125.8 (d), 124.9 (d), 119.9 (d), 66.7 (t), 61.9 (s), 47.1 (d), 23.0 (q). IR $\left(\mathrm{CHCl}_{3}\right.$, $\left.\mathrm{cm}^{-1}\right)$ 3500-2490, 3406, 3304, 3064, 2950, 2899, 2617, 2527, 1719. LRMS ( $\mathrm{m} / \mathrm{z}$, relative intensity) $388\left(\mathrm{MH}^{+}, 5\right), 178(100), 166(50), 120$ (48). HRMS calcd for $\mathrm{C}_{24} \mathrm{H}_{22} \mathrm{NO}_{4}$ : 388.1549, found: $388.1540 .[\alpha]_{\mathrm{D}}{ }^{20}=-22.7\left(c=1.16, \mathrm{CHCl}_{3}\right)$. 
(S)-2-((9H-Fluoren-9-yl)methylcarbamate)-2-phenylpropanoic acid (24e)<smiles>C[C@](NC(=O)OCC1c2ccccc2-c2ccccc21)(C(=O)O)c1ccccc1</smiles>

Prepared as per 15d from 23e. The resulting light yellow solid was purified by flash chromatography (5\% of methanol in dichloromethane) to yield pure $(S)$-Fmoc amino acid 24e (34 mg, 90\%, 2 steps, white solid). $88 \%$ ee (measured by HPLC). m.p.: $91-93{ }^{\circ} \mathrm{C}{ }^{\mathbf{1}} \mathbf{H}$ NMR $\left(300 \mathrm{MHz}, \mathrm{CD}_{3} \mathrm{OD}\right) 7.78(\mathrm{~d}, 2 \mathrm{H}, J=7.7 \mathrm{~Hz}), 7.66-7.64(\mathrm{~m}, 2 \mathrm{H}), 7.50-7.40(\mathrm{~m}, 2 \mathrm{H})$, 7.40-7.22 (m, 7H), 4.30-4.28 (m, 2H), 4.25-4.15 (m, 1H), $1.92(\mathrm{~s}, 3 \mathrm{H}) .{ }^{13} \mathrm{C}$ NMR $(75.5$ $\left.\mathrm{MHz}, \mathrm{CDCl}_{3}\right) \delta(\mathrm{ppm}) 176.6(\mathrm{~s}), 155.2(\mathrm{~s}), 143.7(\mathrm{~s}), 141.2(\mathrm{~s}), 128.6$ (d), $127.6(\mathrm{~d}), 127.0$ (d), 125.7 (d), 124.9 (d), 119.9 (d), 66.6 (t), 62.1 (s), 47.1 (d), 23.4 (q). IR $\left(\mathrm{CHCl}_{3}, \mathrm{~cm}^{-1}\right)$ 3540-2220 (br), 3401, 3302, 3063, 3017, 2948, 1701, 1651, 1502, 1451. LRMS (m/z, relative intensity) $388\left(\mathrm{MH}^{+}, 3\right), 179$ (83), 178 (88), $166(100), 120$ (92). HRMS calcd for $\mathrm{C}_{24} \mathrm{H}_{22} \mathrm{NO}_{4}\left(\mathrm{MH}^{+}\right): 388.1549$, found: $388.1540 .[\alpha]_{\mathrm{D}}{ }^{20}=18.2\left(c=1.19, \mathrm{CHCl}_{3}\right)$.

(9H-Fluoren-9-yl)methyl (R)-2-formylpentan-2-ylcarbamate (26d)<smiles>CCC[C@H](C)NC(=O)OCC1c2ccccc2-c2ccccc21</smiles>

(R)-Aldehyde (71.4 mg, 87\%, colorless oil). ${ }^{1} \mathbf{H}$ NMR (300 MHz, $\left.\mathrm{CDCl}_{3}\right) 9.34(\mathrm{~s}, 1 \mathrm{H})$, $7.77(\mathrm{~d}, 2 \mathrm{H}, J=7.2 \mathrm{~Hz}), 7.60(\mathrm{~d}, 2 \mathrm{H}, J=7.2 \mathrm{~Hz}), 7.41(\mathrm{t}, 2 \mathrm{H}, J=7.2 \mathrm{~Hz}), 7.32(\mathrm{t}, 2 \mathrm{H}, J=$ $7.2 \mathrm{~Hz}$ ), 5.49 (br s, 1H), 4.43 (br d, $2 \mathrm{H}, J=5.0 \mathrm{~Hz}), 4.21$ (t, $1 \mathrm{H}, J=6.6 \mathrm{~Hz}), 1.99$ (br td, $\left.1 \mathrm{H}, J_{1}=13.0, J_{2}=4.2\right), 1.67\left(\mathrm{td}, 1 \mathrm{H}, J_{1}=12.6, J_{2}=5.0\right), 1.40(\mathrm{~s}, 3 \mathrm{H}), 1.34-1.02(\mathrm{~m}, 2 \mathrm{H})$, 0.94-0.88 (m, 3H). ${ }^{13} \mathrm{C}$ NMR (75.5 MHz, $\left.\mathrm{CDCl}_{3}\right) \delta(\mathrm{ppm}) 200.4(\mathrm{~d}), 154.9(\mathrm{~s}), 143.8(\mathrm{~s})$, 141.4 (s), 127.7 (d), 127.1 (d), 124.9 (d), 120.0 (d), 66.4 (t), 62.6 (s), 47.2 (d), 36.4 (t), 20.0 (q), 16.8 (t), 14.1 (q). IR (neat, $\left.\mathrm{cm}^{-1}\right)$ 3018, 2960, 2873, 2814, 2709, 1718. LRMS (m/z, relative intensity) $337\left(\mathrm{M}^{+}, 2\right), 308\left((\mathrm{M}-\mathrm{CHO})^{+}, 12\right), 179$ (100), 178 (93). HRMS calcd for $\mathrm{C}_{21} \mathrm{H}_{23} \mathrm{NO}_{3}: 337.1678$, found: 337.1672 .

(9H-Fluoren-9-yl)methyl (S)-2-formylpentan-2-ylcarbamate (27d)<smiles>CCC[C@H](C)NC(=O)OCC1c2ccccc2-c2ccccc21</smiles>

(S)-Aldehyde (86\%, colorless oil). ${ }^{1} \mathbf{H}$ NMR $\left(300 \mathrm{MHz}, \mathrm{CDCl}_{3}\right) 9.33$ (s, 1H), 7.77 (d, 2H, $J=7.2 \mathrm{~Hz}), 7.60$ (d, 2H, $J=7.2 \mathrm{~Hz}), 7.41$ (t, $2 \mathrm{H}, J=7.4 \mathrm{~Hz}), 7.32$ (t, 2H, $J=6.9 \mathrm{~Hz}), 5.46$ (br s, 1H), 4.42 (br d, $2 \mathrm{H}, J=6.1 \mathrm{~Hz}), 4.21(\mathrm{t}, 1 \mathrm{H}, J=6.3 \mathrm{~Hz}), 2.00\left(\mathrm{td}, 1 \mathrm{H}, J_{1}=13.2, J_{2}=\right.$ 4.7), $1.66\left(\mathrm{td}, 1 \mathrm{H}, J_{1}=12.9, J_{2}=4.4\right), 1.41(\mathrm{~s}, 3 \mathrm{H}), 1.26-1.19(\mathrm{~m}, 1 \mathrm{H}), 1.16-1.05(\mathrm{~m}, 1 \mathrm{H})$, 
0.93-0.88 (m, 3H).. ${ }^{13} \mathrm{C}$ NMR (75.5 MHz, $\left.\mathrm{CDCl}_{3}\right) \delta(\mathrm{ppm}) 200.4$ (d), $154.8(\mathrm{~s}), 143.8$ (s), 141.4 (s), 127.7 (d), 127.0 (d), 124.9 (d), 120.0 (d), 66.4 (t), 62.6 (s), 47.2 (d), 36.4 (t), 20.1 (q), 16.8 (t), 14.1 (q). IR (neat, $\left.\mathrm{cm}^{-1}\right)$ 3337, 2960, 2873, 1711, 1515. LRMS (m/z, relative intensity) $337\left(\mathrm{M}^{+}, 2\right), 308\left((\mathrm{M}-\mathrm{CHO})^{+}, 22\right), 179$ (97), 178 (100), 86 (75), 84 (76). HRMS calcd for $\mathrm{C}_{21} \mathrm{H}_{23} \mathrm{NO}_{3}$ : 337.1678 , found: 337.1672 .

(9H-Fluoren-9-yl)methyl (R)-1-formyl-1-phenylethylcarbamate (26e)<smiles>C[C@@](NC(=O)OCC1c2ccccc2-c2ccccc21)(C(=O)O)c1ccccc1</smiles>

(R)-Aldehyde (colorless oil). ${ }^{1} \mathbf{H}$ NMR $\left(300 \mathrm{MHz}, \mathrm{CDCl}_{3}\right) 9.26(\mathrm{~s}, 1 \mathrm{H}), 7.78(\mathrm{~d}, 2 \mathrm{H}, J=$ $7.7 \mathrm{~Hz}), 7.60(\mathrm{~d}, 2 \mathrm{H}, J=7.7 \mathrm{~Hz}), 7.44-7.26(\mathrm{~m}, 9 \mathrm{H}), 6.09(\mathrm{~s}, 1 \mathrm{H}), 4.42(\mathrm{~d}, 2 \mathrm{H}, J=6.1 \mathrm{~Hz})$, $4.21(\mathrm{t}, 1 \mathrm{H}, J=6.3 \mathrm{~Hz}), 1.89(\mathrm{~s}, 3 \mathrm{H})$.

(9H-Fluoren-9-yl)methyl (S)-1-formyl-1-phenylethylcarbamate (27e)<smiles>C[C@@](NC(=O)OCC1c2ccccc2-c2ccccc21)(C(=O)O)c1ccccc1</smiles>

(S)-Aldehyde (colorless oil). ${ }^{1} \mathbf{H}$ NMR $\left(300 \mathrm{MHz}, \mathrm{CDCl}_{3}\right) 9.26(\mathrm{~s}, 1 \mathrm{H}), 7.77$ (d, 2H, $J=$ $7.2 \mathrm{~Hz}$ ), 7.59 (d, 2H, J = 7.7 Hz), 7.43-7.19 (m, 9H), 6.09 (s, 1H), 4.42 (d, 2H, $J=6.6 \mathrm{~Hz})$, $4.21(\mathrm{t}, 1 \mathrm{H}, J=6.3 \mathrm{~Hz}), 1.89(\mathrm{~s}, 3 \mathrm{H})$.

$N$-((R,E)-1-((1S,2S,5R)-2-Isopropyl-5-methylcyclohexyl)-3-methylhex-1-en-3yl)acrylamide (17)

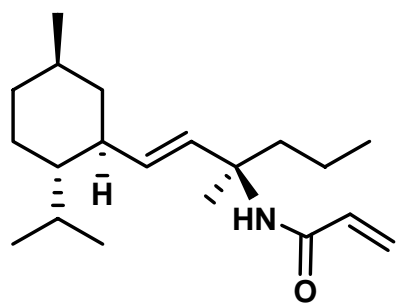

To a solution of isocyanate $6 \mathbf{d}(44 \mathrm{mg}, 0.16 \mathrm{mmol})$ in THF $(1.1 \mathrm{~mL}, 0.15 \mathrm{M})$ at $0{ }^{\circ} \mathrm{C}$ was added vinyl magnesium bromide $(0.85 \mathrm{M}$ in THF, $0.2 \mathrm{~mL}, 0.17 \mathrm{mmol})$. The solution was stirred at $0{ }^{\circ} \mathrm{C}$ for $2 \mathrm{~h}$. The reaction mixture was then poured over a saturated aqueous solution of ammonium chloride. The solution was extracted three times with diethyl ether $(5 \mathrm{~mL})$. The organic layers were combined, washed with brine, dried with $\mathrm{MgSO}_{4}$, filtered and concentrated under reduced pressure. The resulting oil was purified by flash chromatography ( $30 \%$ of diethyl ether in hexanes) to yield pure $(R)$-acrylamide 17 (46 mg, 96\%, colorless oil). ${ }^{\mathbf{1}} \mathbf{H}$ NMR $\left(300 \mathrm{MHz}, \mathrm{CDCl}_{3}\right) 6.22\left(\mathrm{dd}, 1 \mathrm{H}, J_{1}=17.1 \mathrm{~Hz}, J_{2}=1.7 \mathrm{~Hz}\right)$, $6.04\left(\mathrm{dd}, 1 \mathrm{H}, J_{1}=17.1 \mathrm{~Hz}, J_{2}=9.9 \mathrm{~Hz}\right), 5.59(\mathrm{~d}, 1 \mathrm{H}, J=15.9 \mathrm{~Hz}), 5.56\left(\mathrm{dd}, 1 \mathrm{H}, J_{1}=9.9\right.$ $\left.\mathrm{Hz}, J_{2}=1.7 \mathrm{~Hz}\right), 5.37(\mathrm{~s}, 1 \mathrm{H}), 5.25\left(\mathrm{dd}, 1 \mathrm{H}, J_{1}=16.0 \mathrm{~Hz}, J_{2}=9.4 \mathrm{~Hz}\right), 1.95-1.65(\mathrm{~m}, 5 \mathrm{H})$, 
1.62-1.56 (m, 2H), $1.44(\mathrm{~s}, 3 \mathrm{H}), 1.42-1.16(\mathrm{~m}, 3 \mathrm{H}), 1.06-0.75(\mathrm{~m}, 7 \mathrm{H}), 0.86(\mathrm{~d}, 3 \mathrm{H}, J=7.2$ $\mathrm{Hz}), 0.85$ (d, 3H, $J=6.6 \mathrm{~Hz}), 0.69$ (d, 3H, $J=7.1 \mathrm{~Hz}) .{ }^{13} \mathrm{C}$ NMR $\left(75.5 \mathrm{MHz}, \mathrm{CDCl}_{3}\right) \delta$ (ppm) 164.4 (s), 134.1 (d), 133.4 (d), 132.1 (d), 125.5 (t), 57.0 (s), 47.2 (d), 44.7 (d), 43.2 $(\mathrm{t}), 42.0(\mathrm{t}), 35.1(\mathrm{t}), 32.4(\mathrm{~d}), 28.1(\mathrm{~d}), 24.6(\mathrm{q}), 24.0(\mathrm{t}), 22.5(\mathrm{q}), 21.4(\mathrm{q}), 17.3(\mathrm{t}), 15.2$ (q), 14.4 (q). IR (neat, $\mathrm{cm}^{-1}$ ) 3287, 3063, 2956, 2928, 2871, 2844, 1659, 1625, 1549. LRMS $\left(\mathrm{m} / \mathrm{z}\right.$, relative intensity) $305\left(\mathrm{M}^{+}, 9\right), 262\left(\left(\mathrm{M}-\mathrm{C}_{3} \mathrm{H}_{7}\right)^{+}, 42\right), 124$ (100). HRMS calcd for $\mathrm{C}_{20} \mathrm{H}_{35} \mathrm{NO}$ : 305.2718 , found: 305.2712 . $[\alpha]_{\mathrm{D}}^{20}=-77.7\left(c=0.91, \mathrm{CHCl}_{3}\right)$.

\section{(R)-5-Methyl-5-propyl-1H-pyrrol-2(5H)-one (18)}

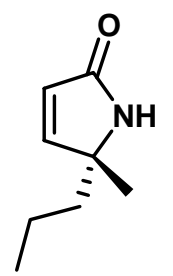

Acrylamide 17 (34.8 mg, $0.114 \mathrm{mmol})$ was dissolved in dichloroethane (23 mL, $0.005 \mathrm{M})$ and argon was bubbled through this solution for $15 \mathrm{~min}$. The mixture was then heat to reflux. The reflux was stopped and the $2^{\text {nd }}$ generation Grubbs catalyst $(9.7 \mathrm{mg}, 10 \mathrm{~mol} \%)$ was added to the solution. The reaction mixture was heat to reflux for $1.5 \mathrm{~h}$. Dichloroethane was then evaporated under reduce pressure. The resulting dark brown oil was purified by flash chromatography ( $80 \%$ ethyl acetate in hexanes) to yield pure dihydropyrrolone $\mathbf{1 8}$ (9.5 mg, 60\%). ${ }^{1} \mathbf{H}$ NMR (300 MHz, $\left.\mathrm{CDCl}_{3}\right) 6.93\left(\mathrm{dd}, 1 \mathrm{H}, J_{1}=5.5 \mathrm{~Hz}, J_{2}=1.7 \mathrm{~Hz}\right), 6.80$ (br s, $1 \mathrm{H}), 5.96\left(\mathrm{dd}, 1 \mathrm{H}, J_{1}=5.5 \mathrm{~Hz}, J_{2}=1.1 \mathrm{~Hz}\right), 1.70-1.52(\mathrm{~m}, 2 \mathrm{H}), 1.40-1.12(\mathrm{~m}, 3 \mathrm{H})$, $1.34(\mathrm{~s}, 3 \mathrm{H}), 0.89(\mathrm{t}, 3 \mathrm{H}, J=7.2 \mathrm{~Hz}) .{ }^{13} \mathrm{C} \mathbf{N M R}\left(75.5 \mathrm{MHz}, \mathrm{CDCl}_{3}\right) \delta(\mathrm{ppm}) 173.1(\mathrm{~s})$, 155.6 (d), 125.2 (d), 64.3 (s), 40.9 (t), 24.2 (q), 17.6 (t), 14.2 (q). IR (neat, $\mathrm{cm}^{-1}$ ) 3214, 2960, 2932, 2873, 1685, 817. LRMS $\left(\mathrm{m} / \mathrm{z}\right.$, relative intensity) $139\left(\mathrm{M}^{+}, 1\right), 124\left(\left(\mathrm{M}_{-} \mathrm{CH}_{3}\right)^{+}\right.$, 3), $96\left(\left(\mathrm{M}-\mathrm{C}_{3} \mathrm{H}_{7}\right)^{+}, 100\right)$. HRMS calcd for $\mathrm{C}_{8} \mathrm{H}_{13} \mathrm{NO}$ : 139.0997, found: 139.0992 . $[\alpha]_{\mathrm{D}}{ }^{20}=$ $-51.2\left(c=0.87, \mathrm{CHCl}_{3}\right)$. 
NMR spectra<smiles>CCC/C=C/[C@@H](O)C1C[C@@H](C)CC[C@H]1C(C)C</smiles>

$8 a$

S- 37

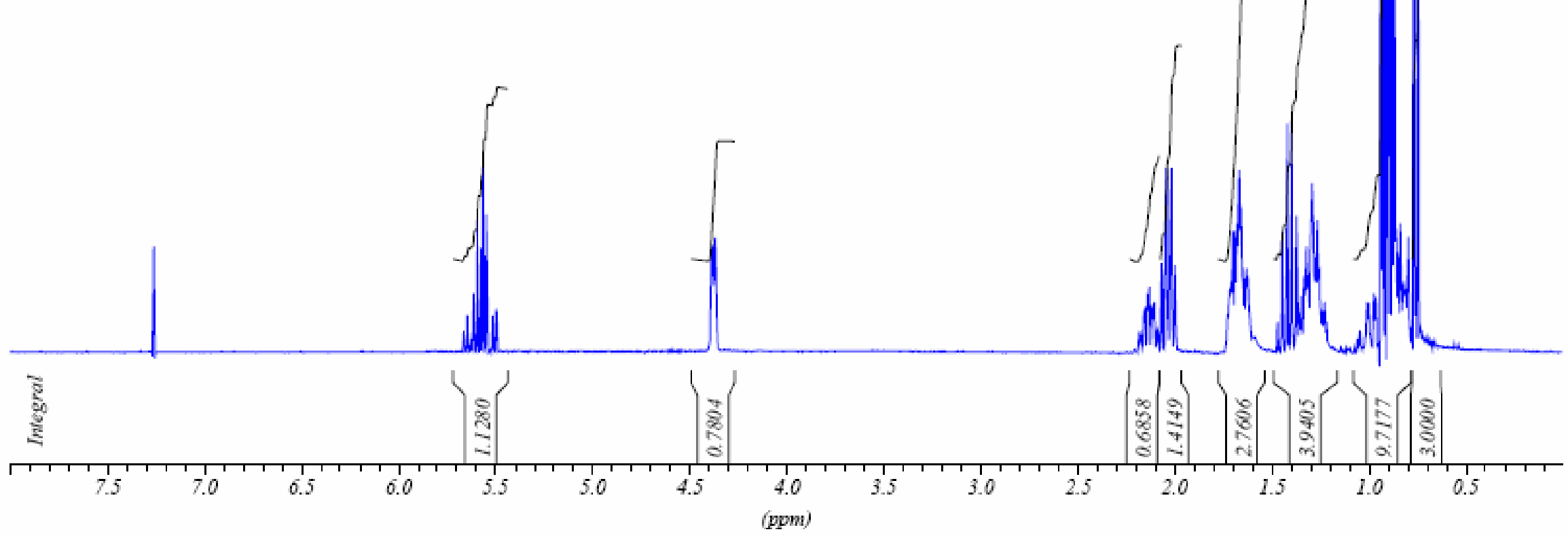




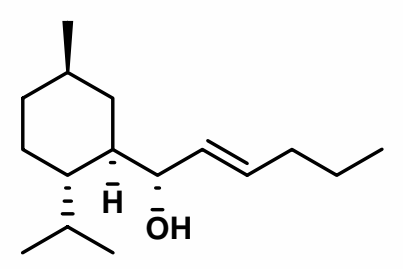

S- 38

$9 a$

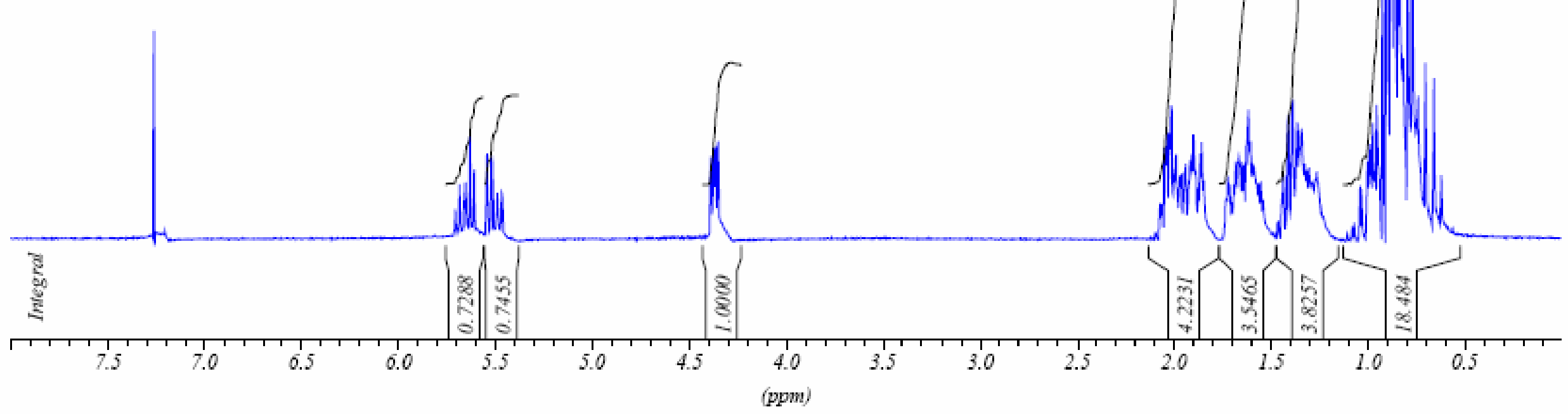




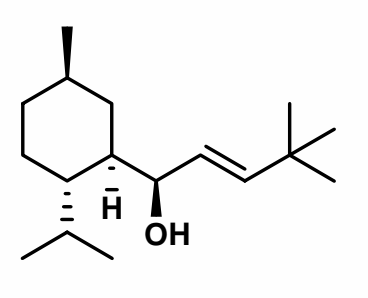

S- 39

$8 b$

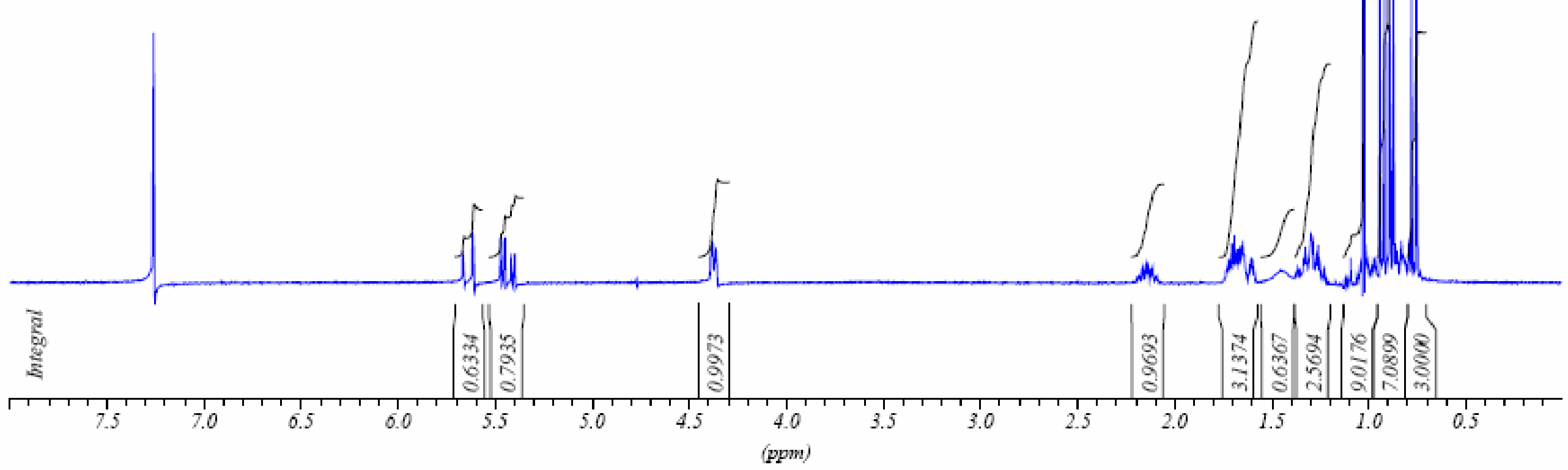




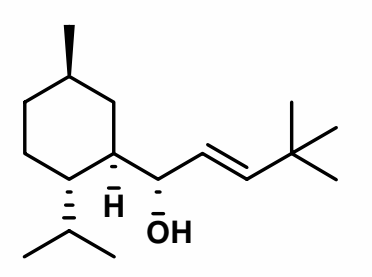

$9 b$

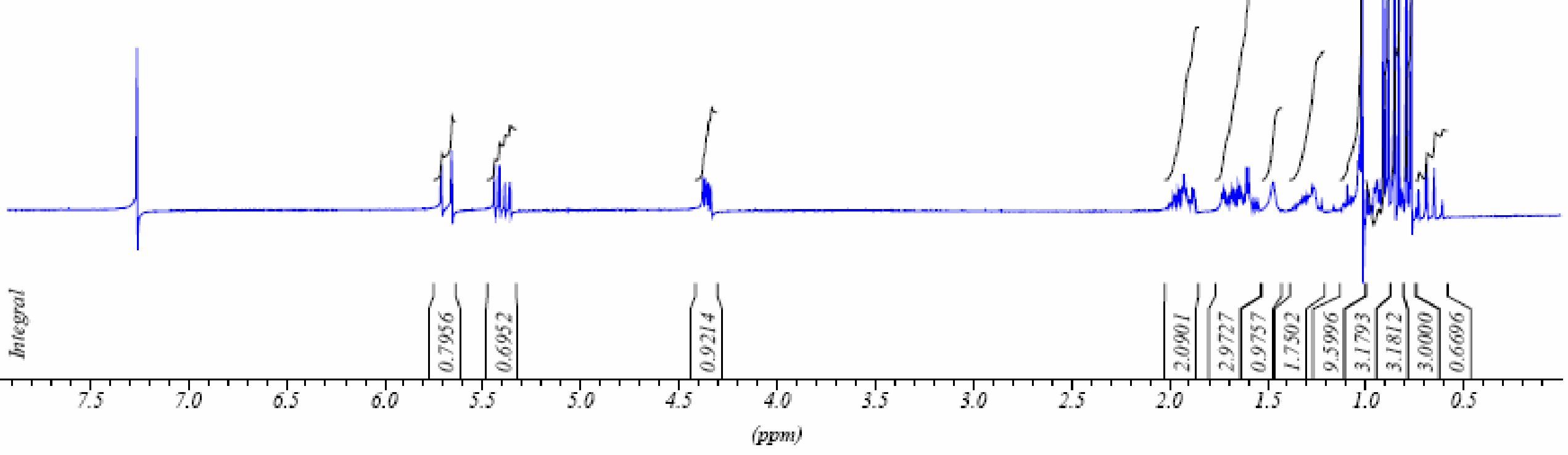




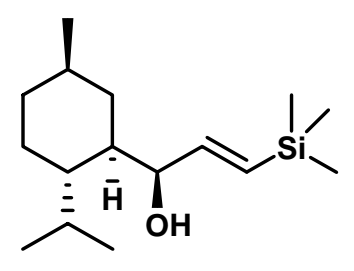

8c

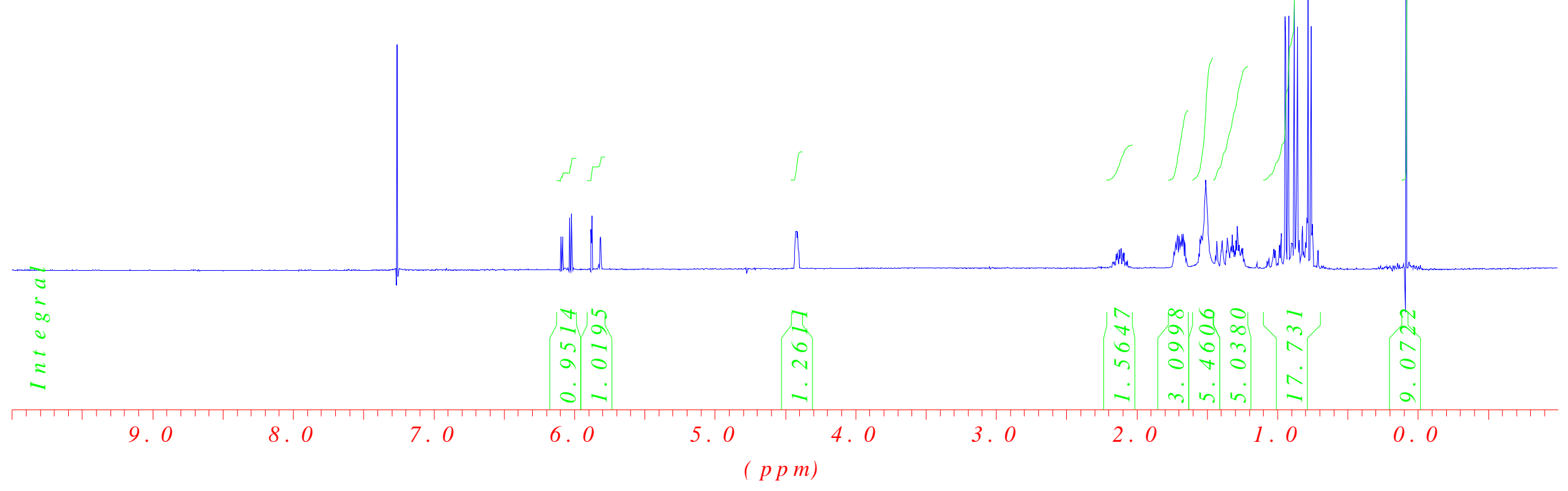




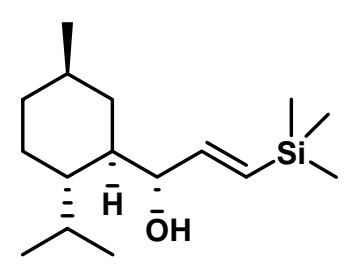

9c

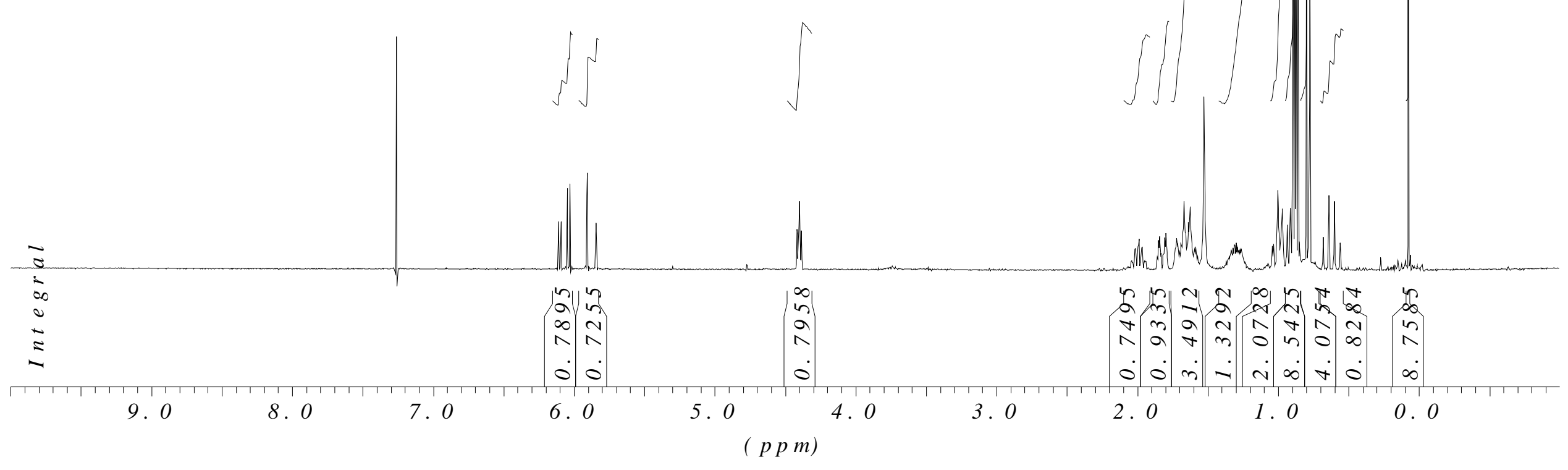




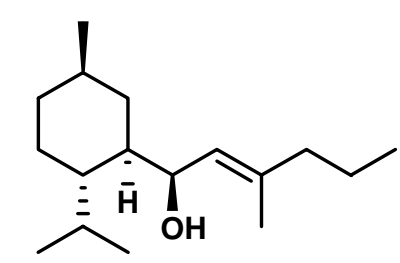

8d

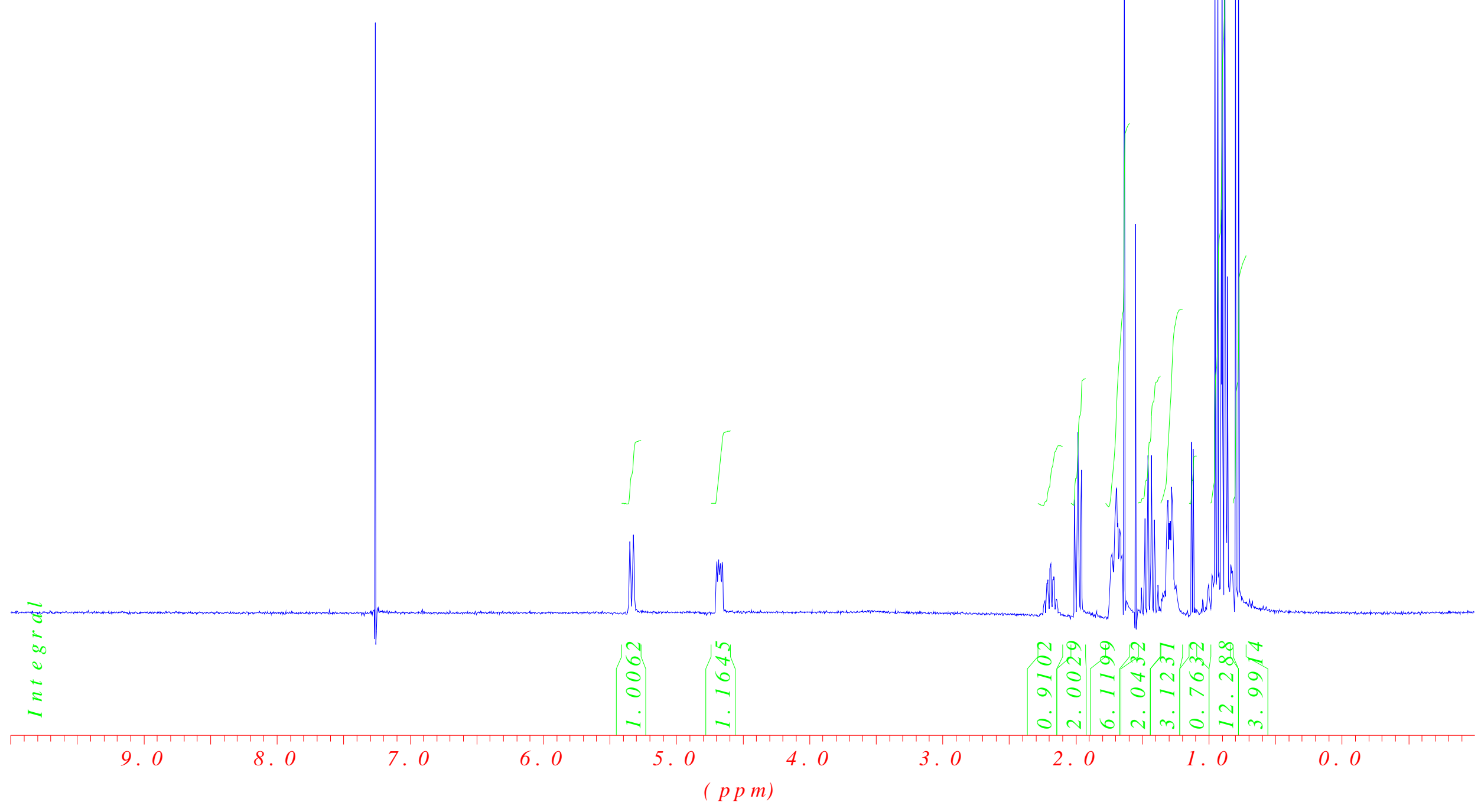




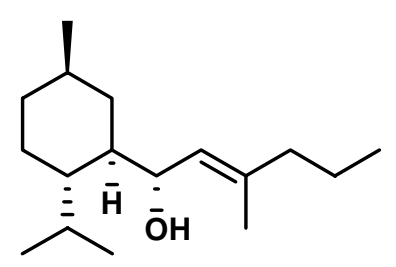

9d

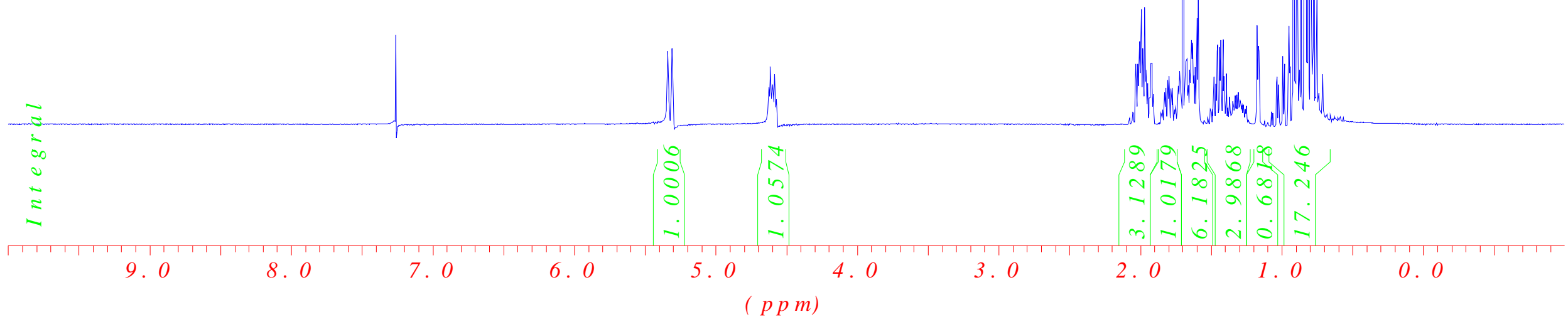


(C)

$8 e$

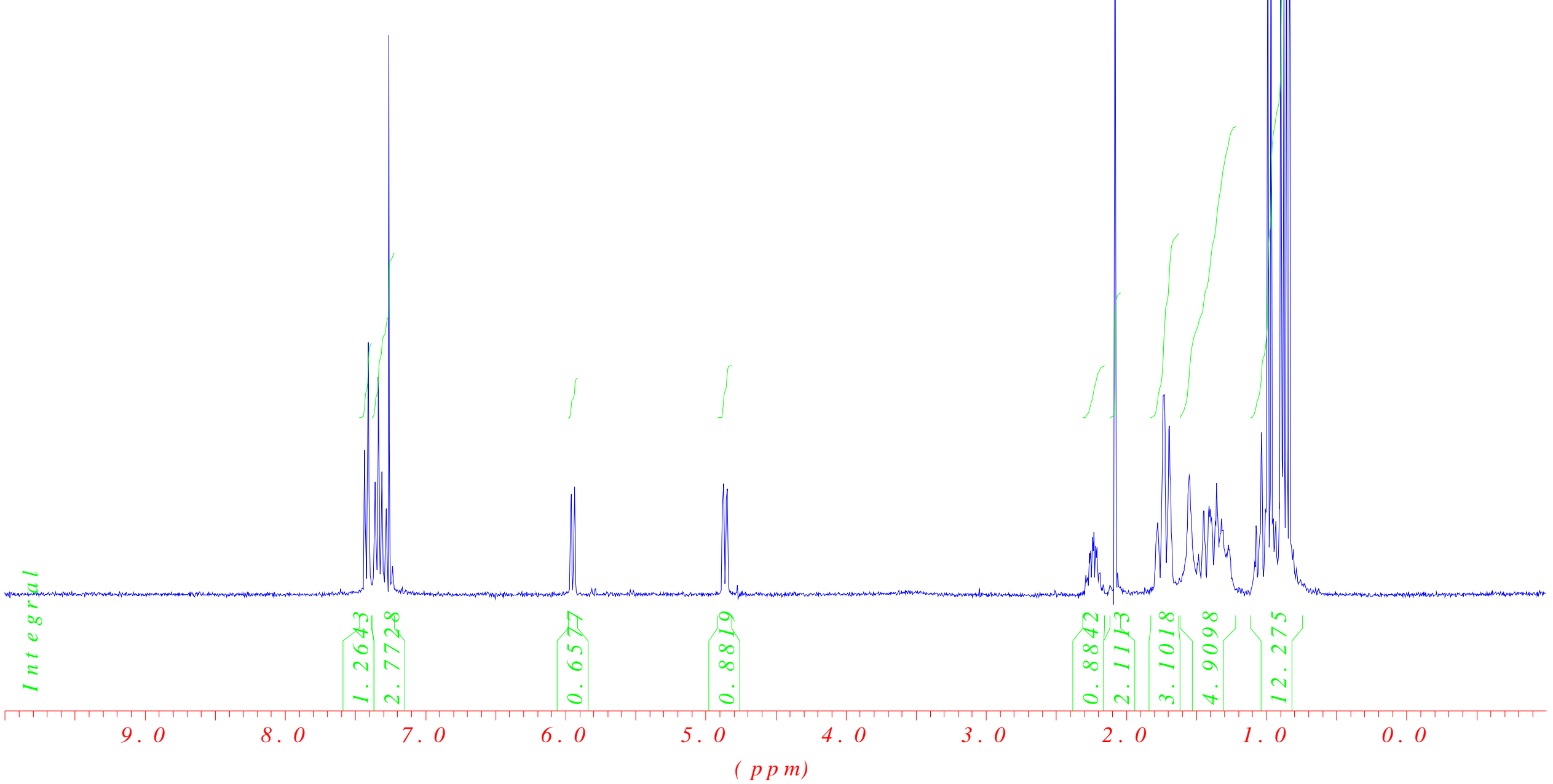




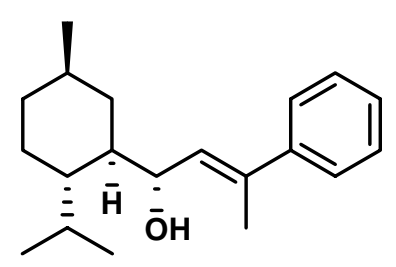

$9 e$

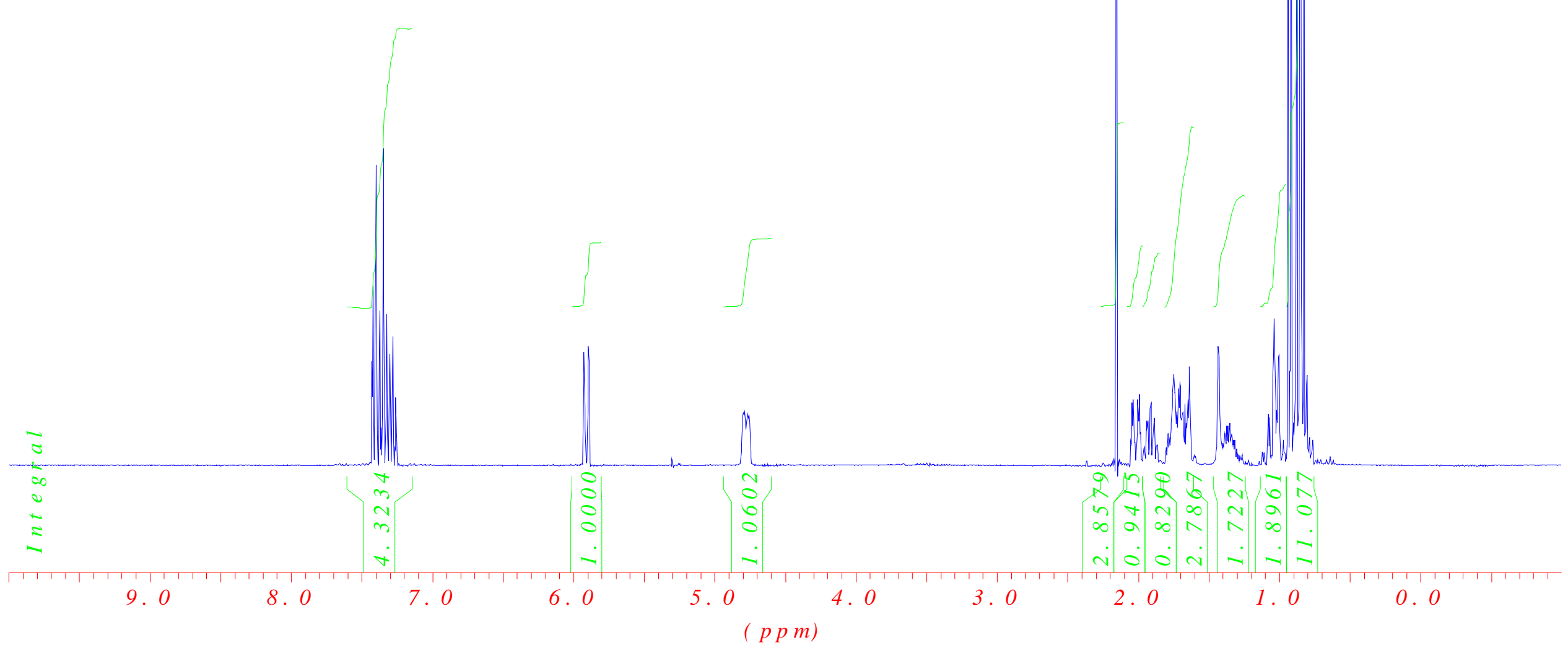




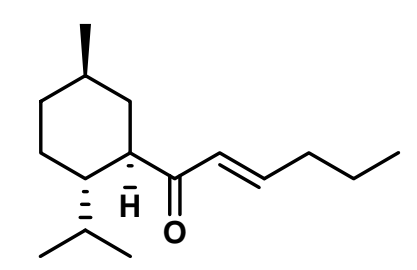

20a

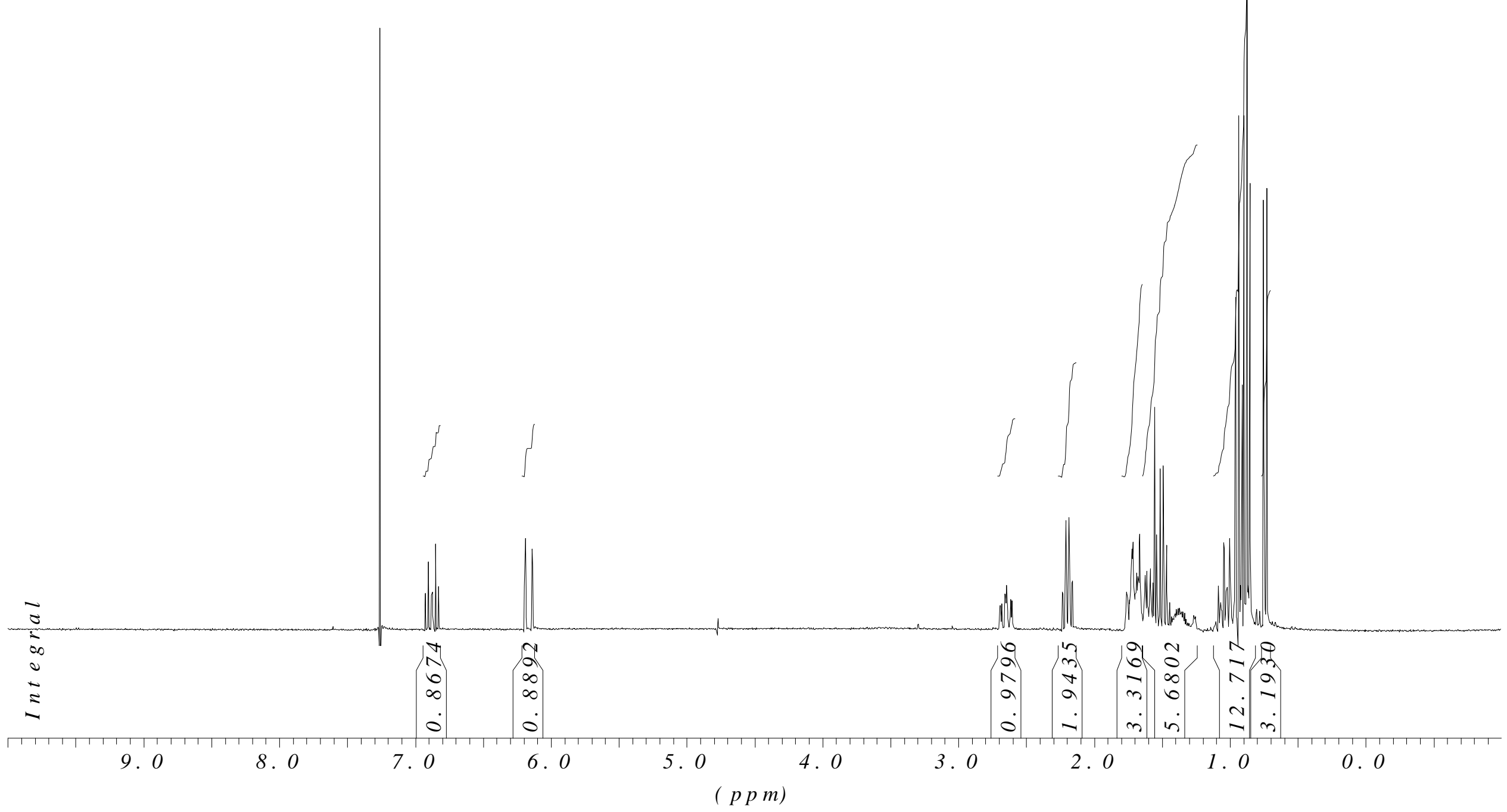




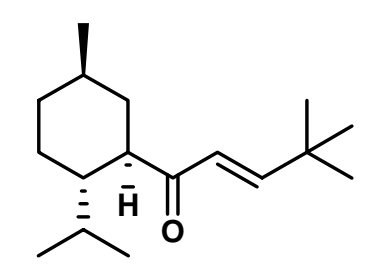

20b

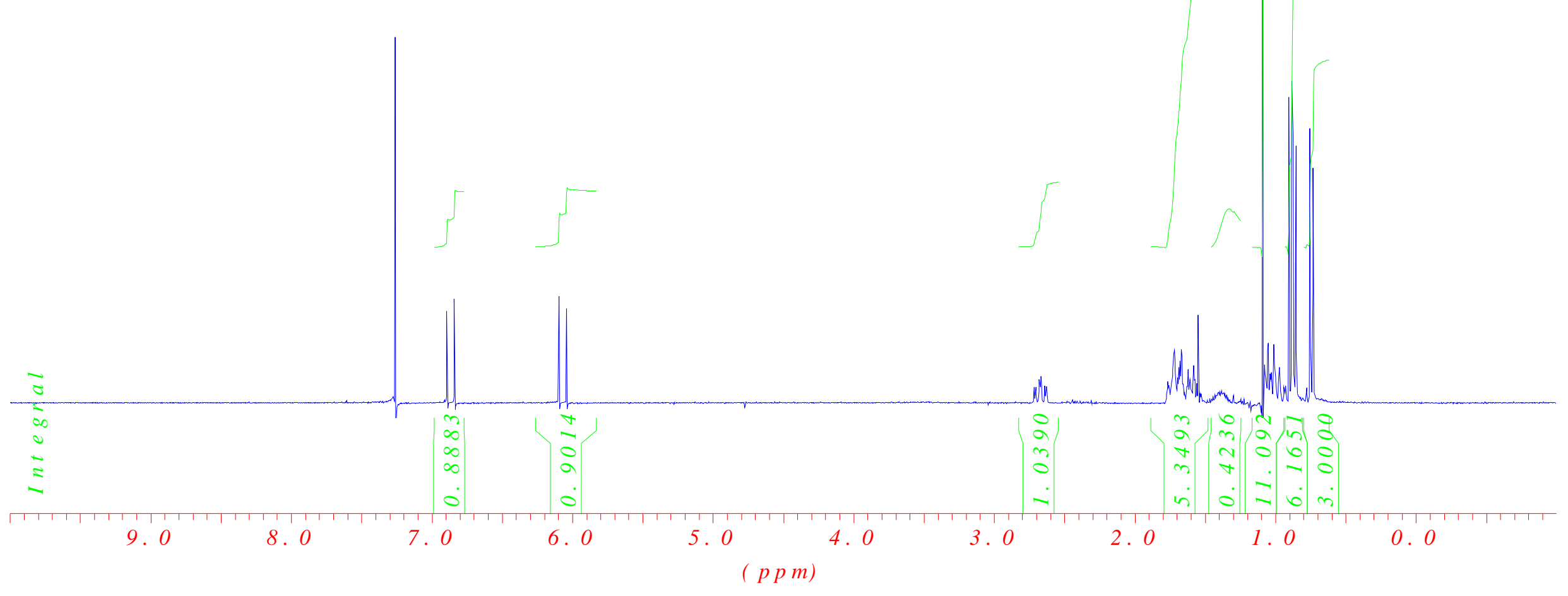




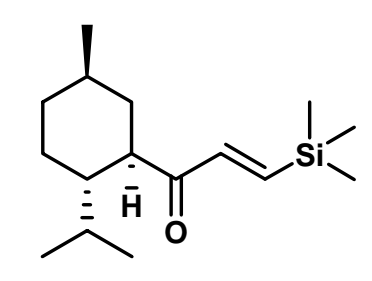

20c

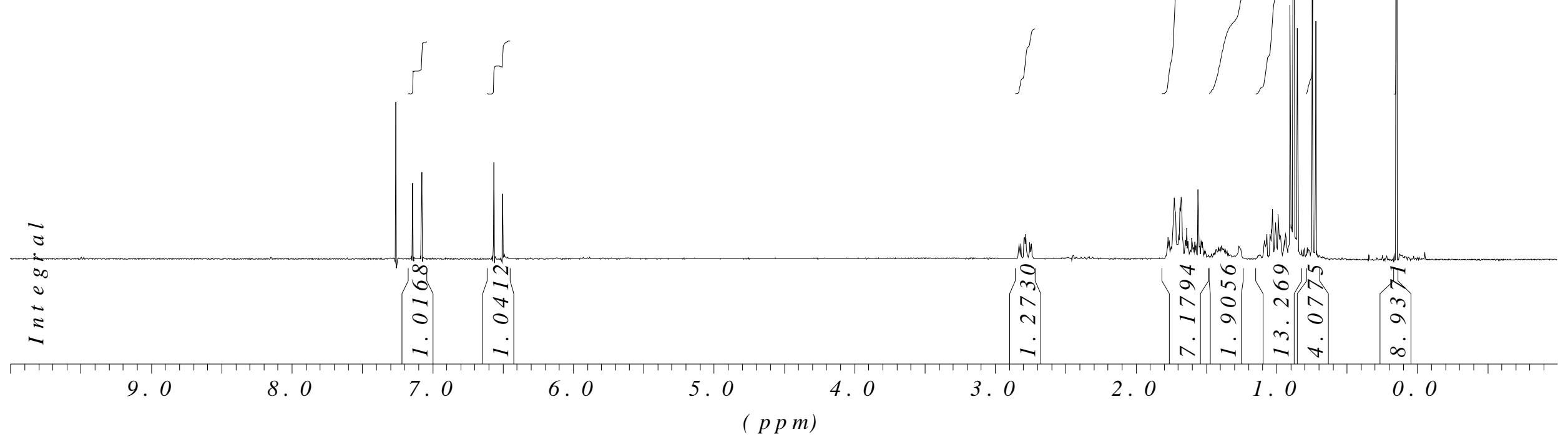




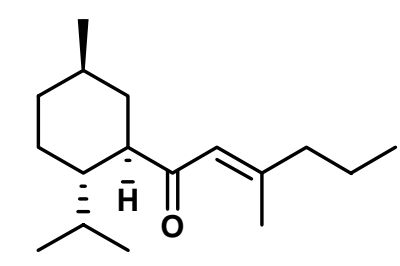

20d

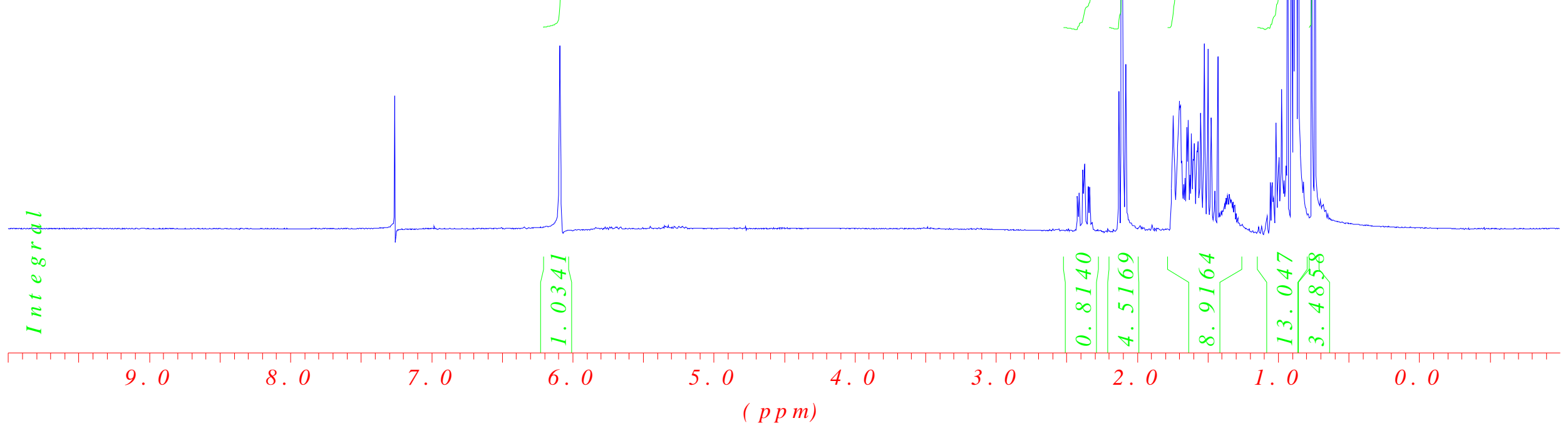


ingos

$20 \mathrm{e}$

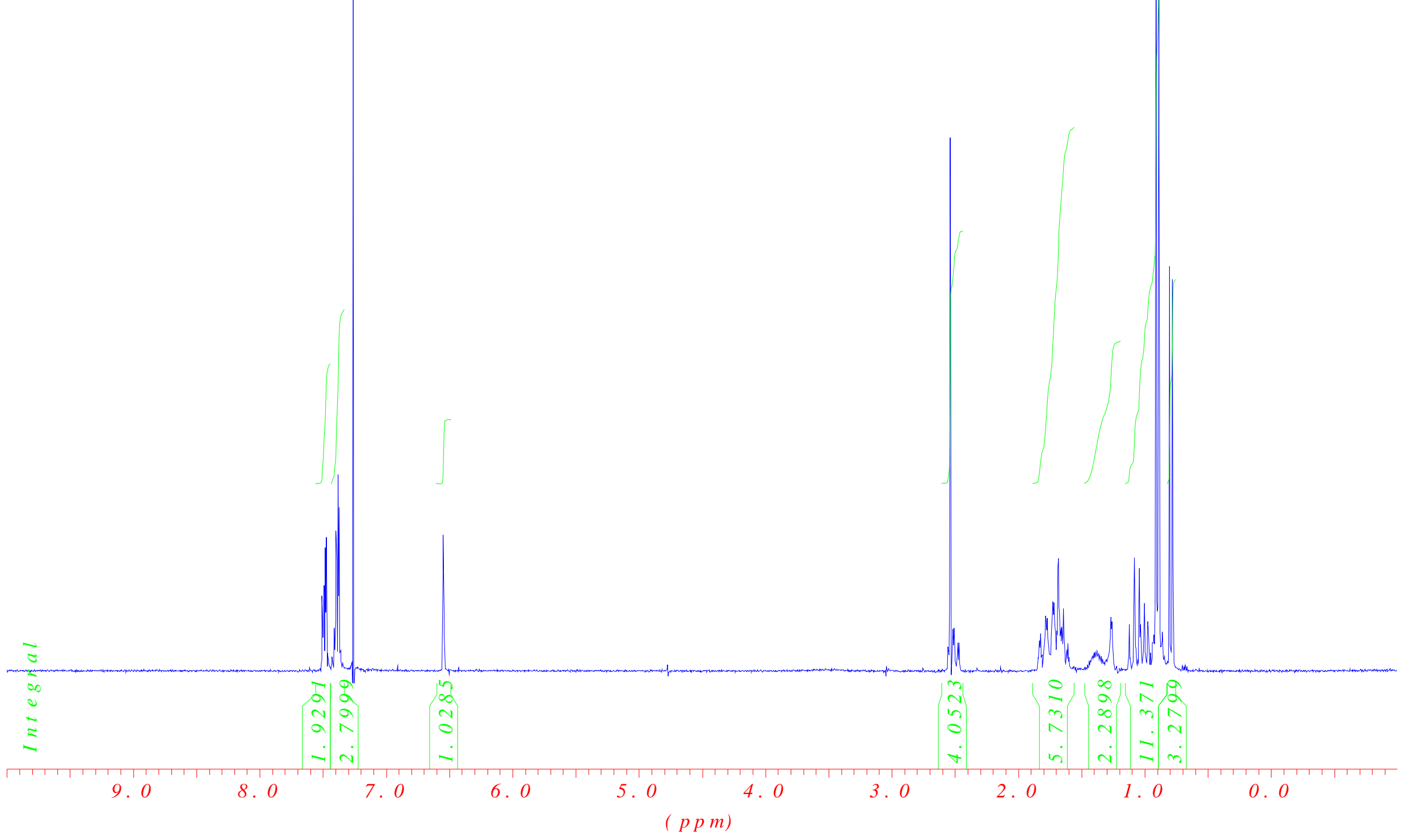



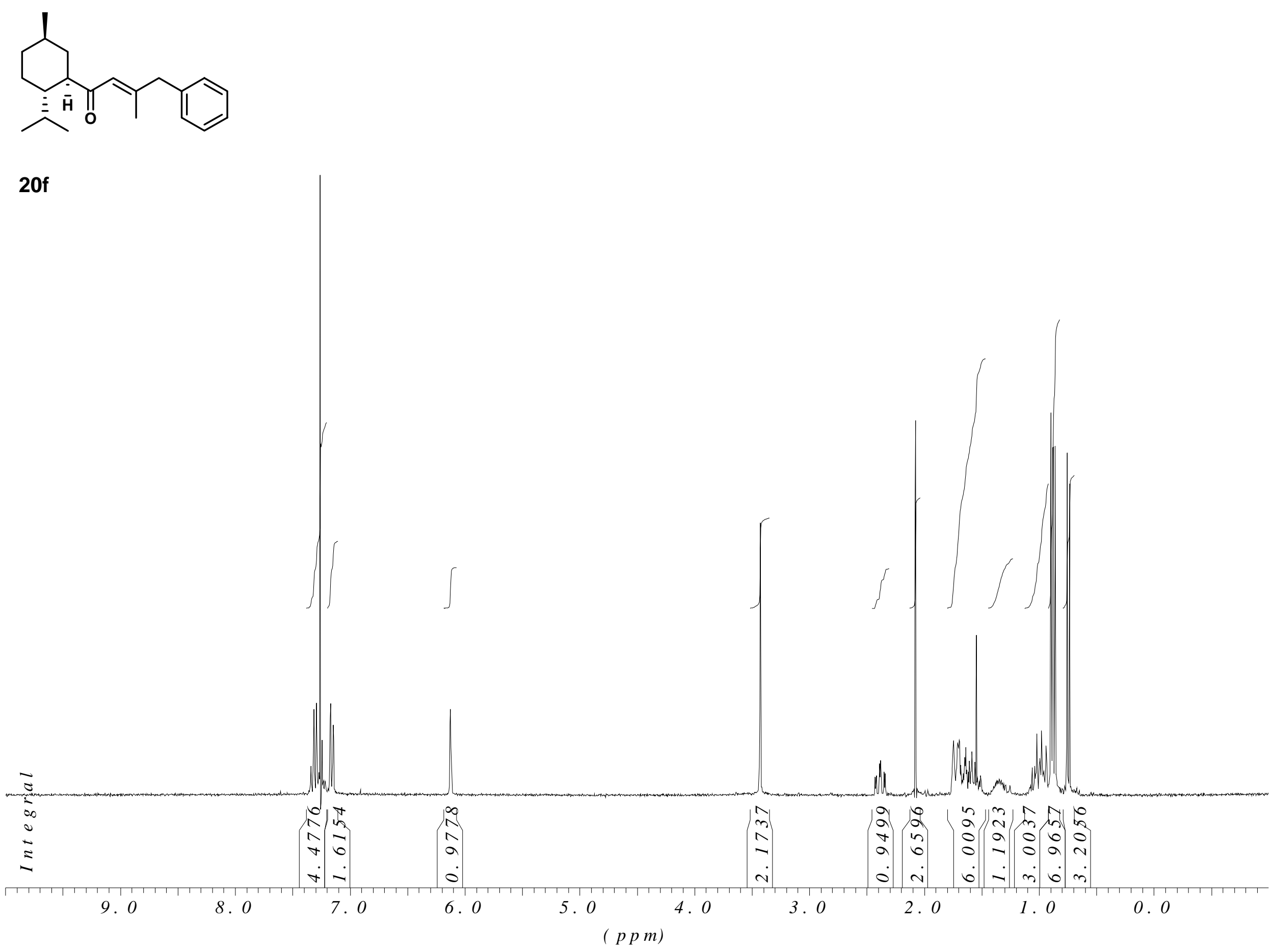


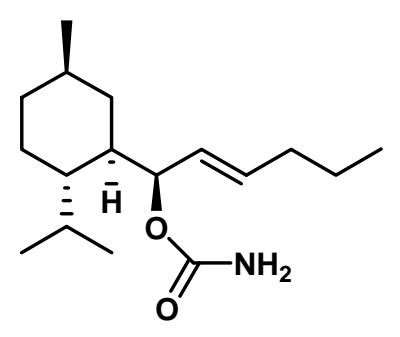

S- 53

$11 a$

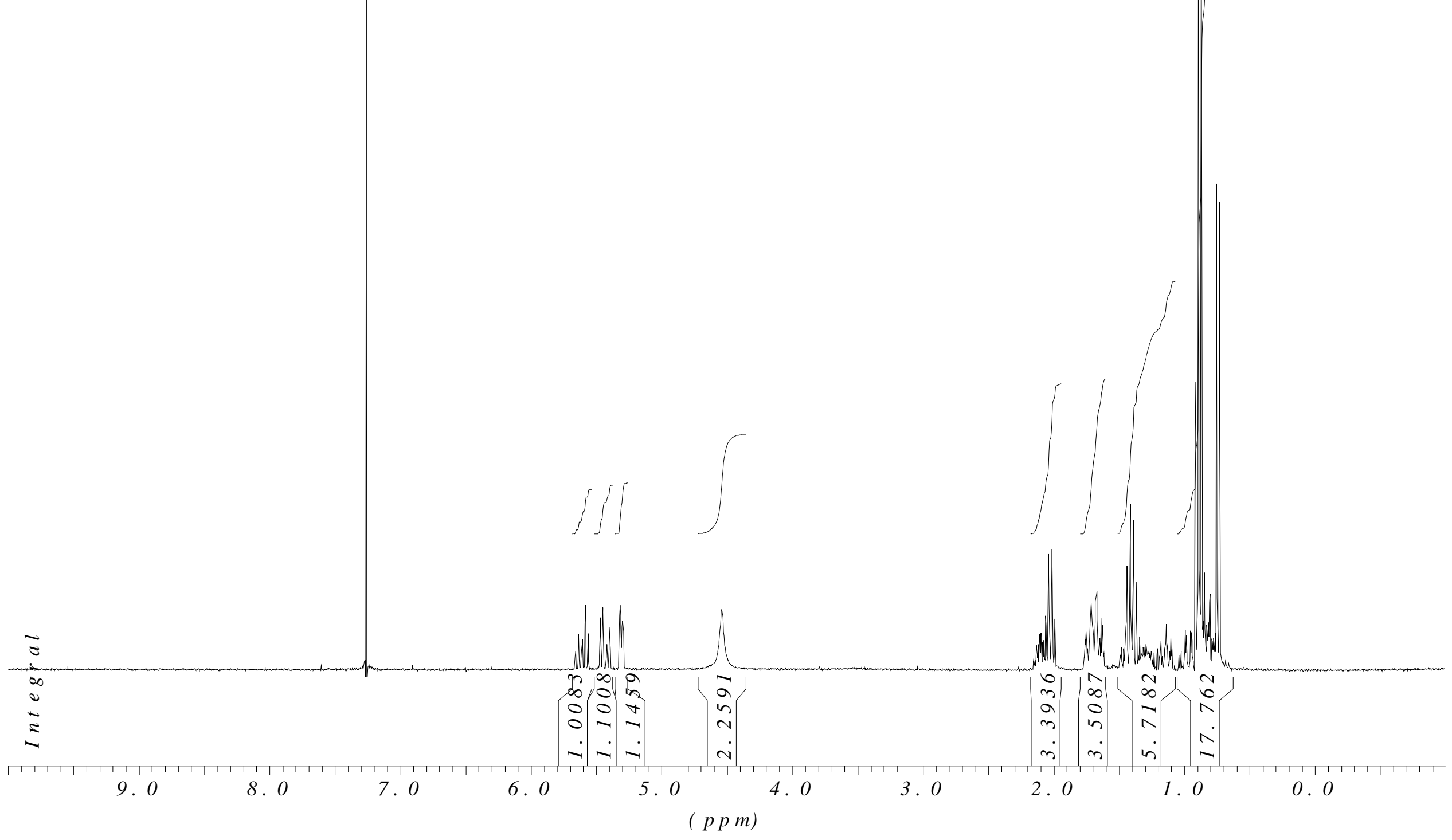




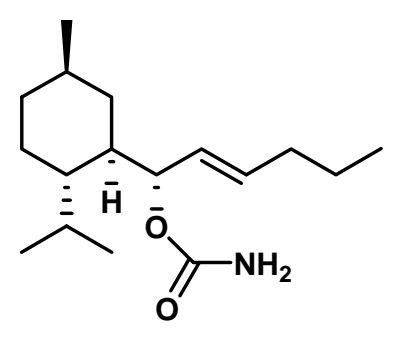

S- 54

$21 a$

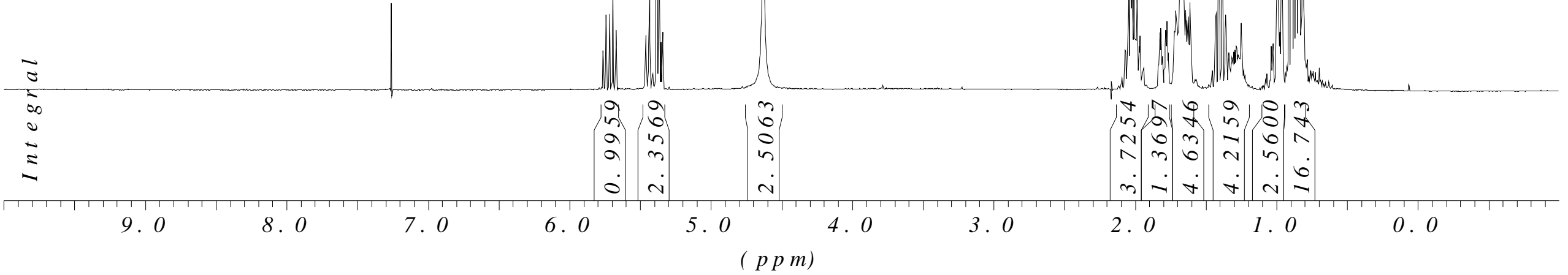




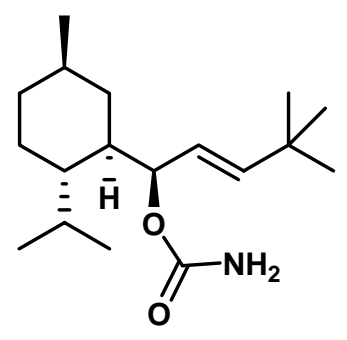

$11 b$

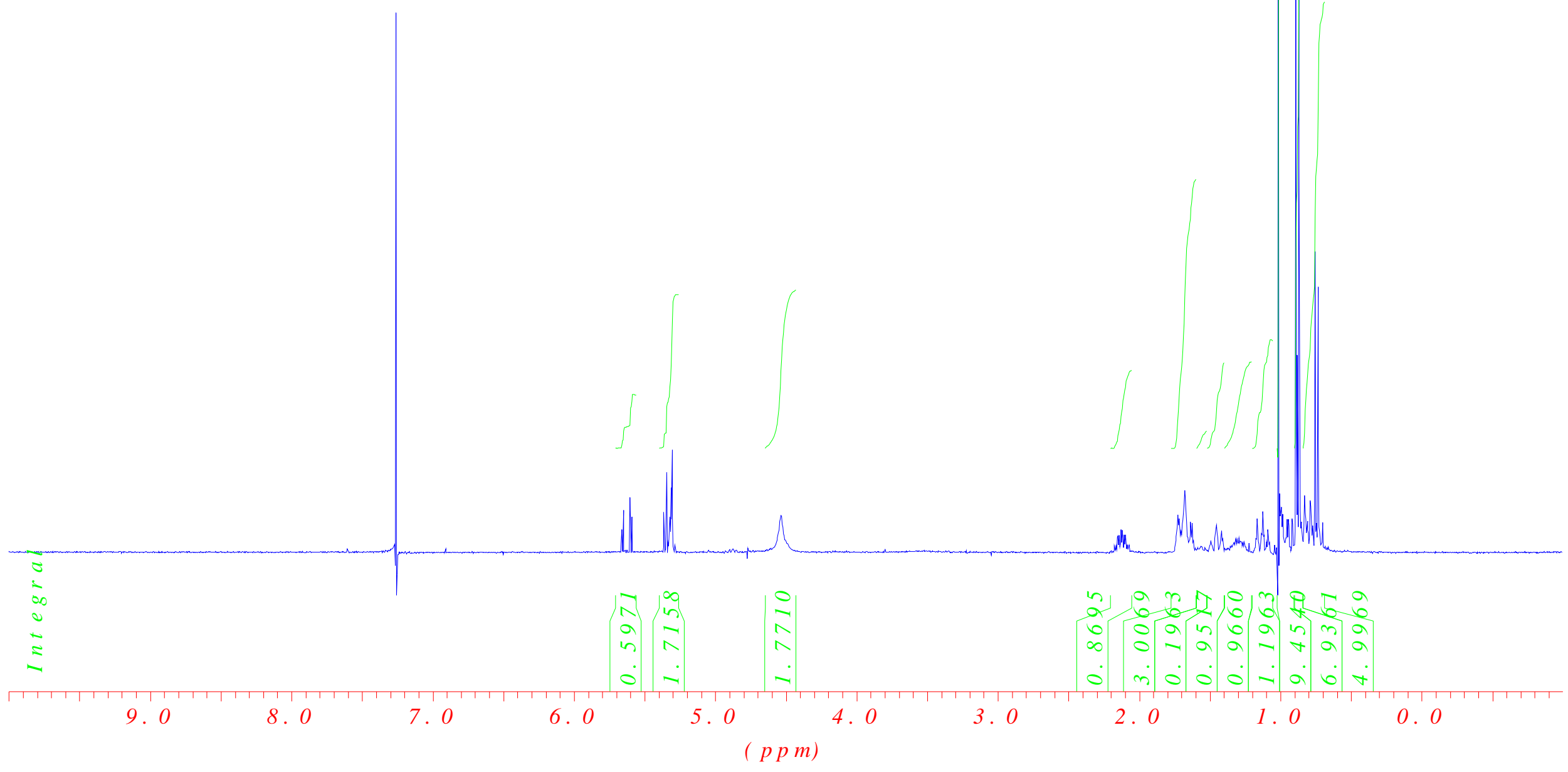




\section{1b}

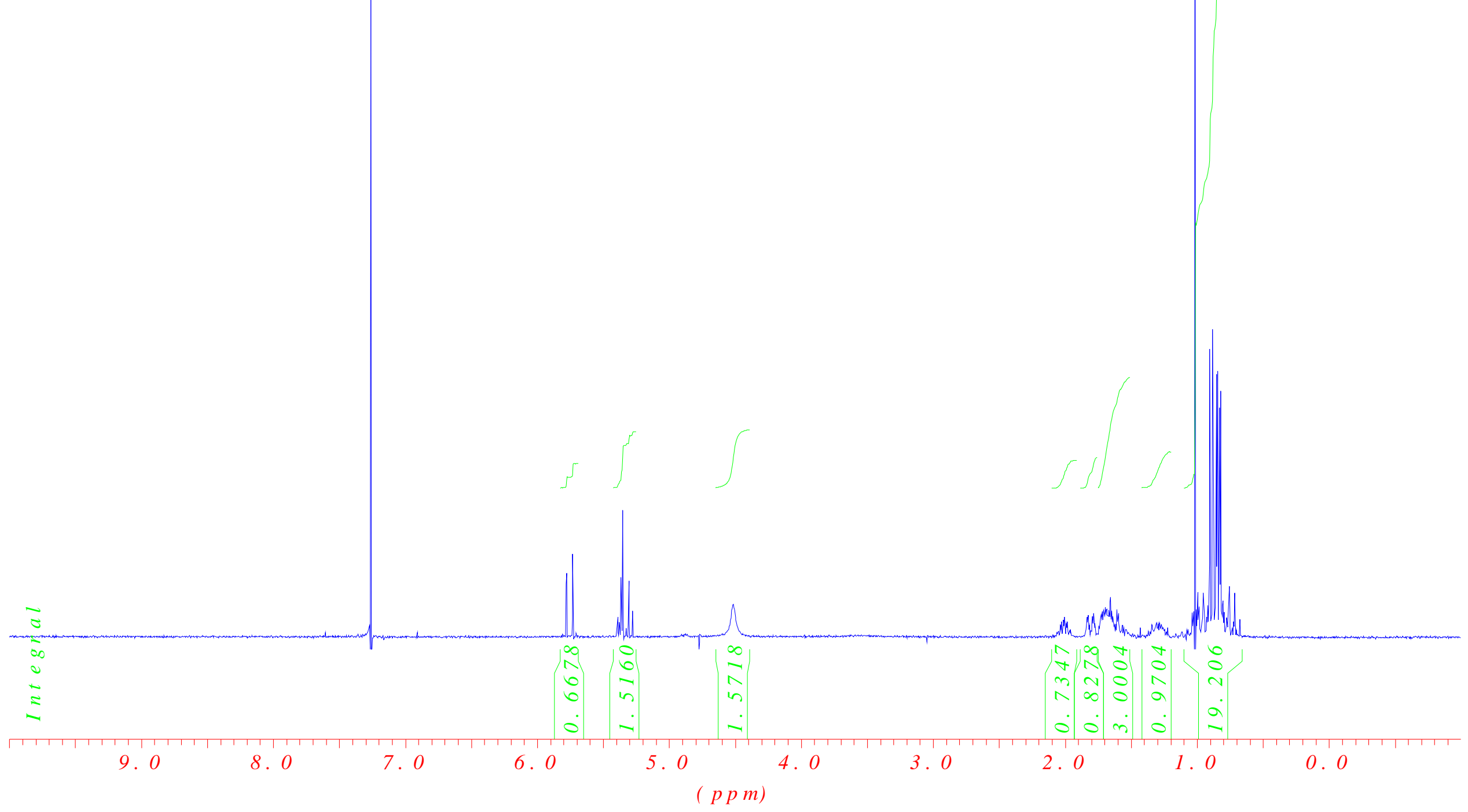




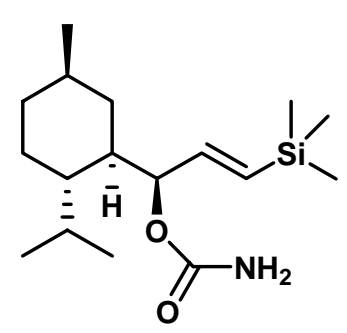

11c

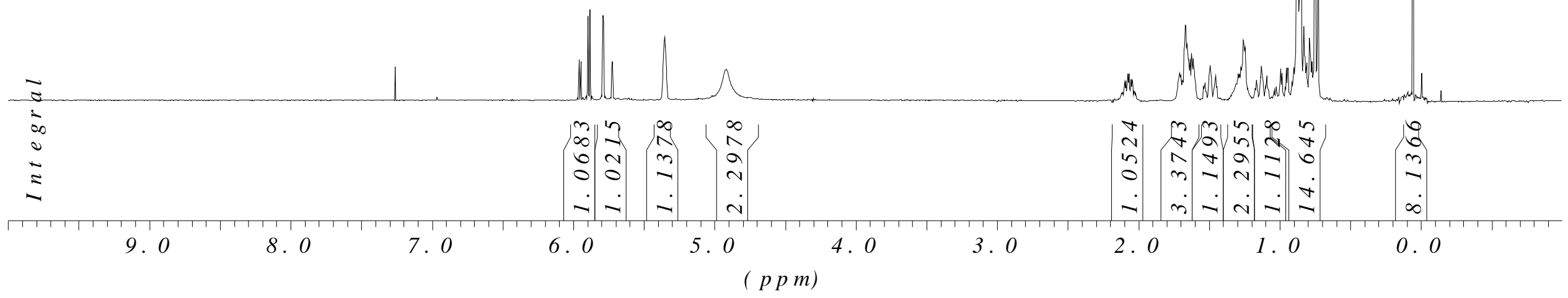




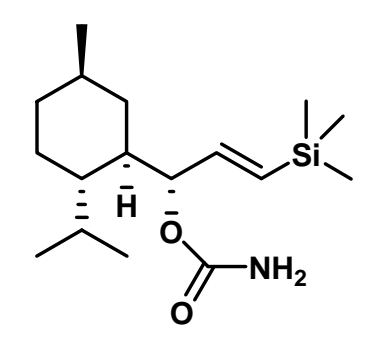

21c

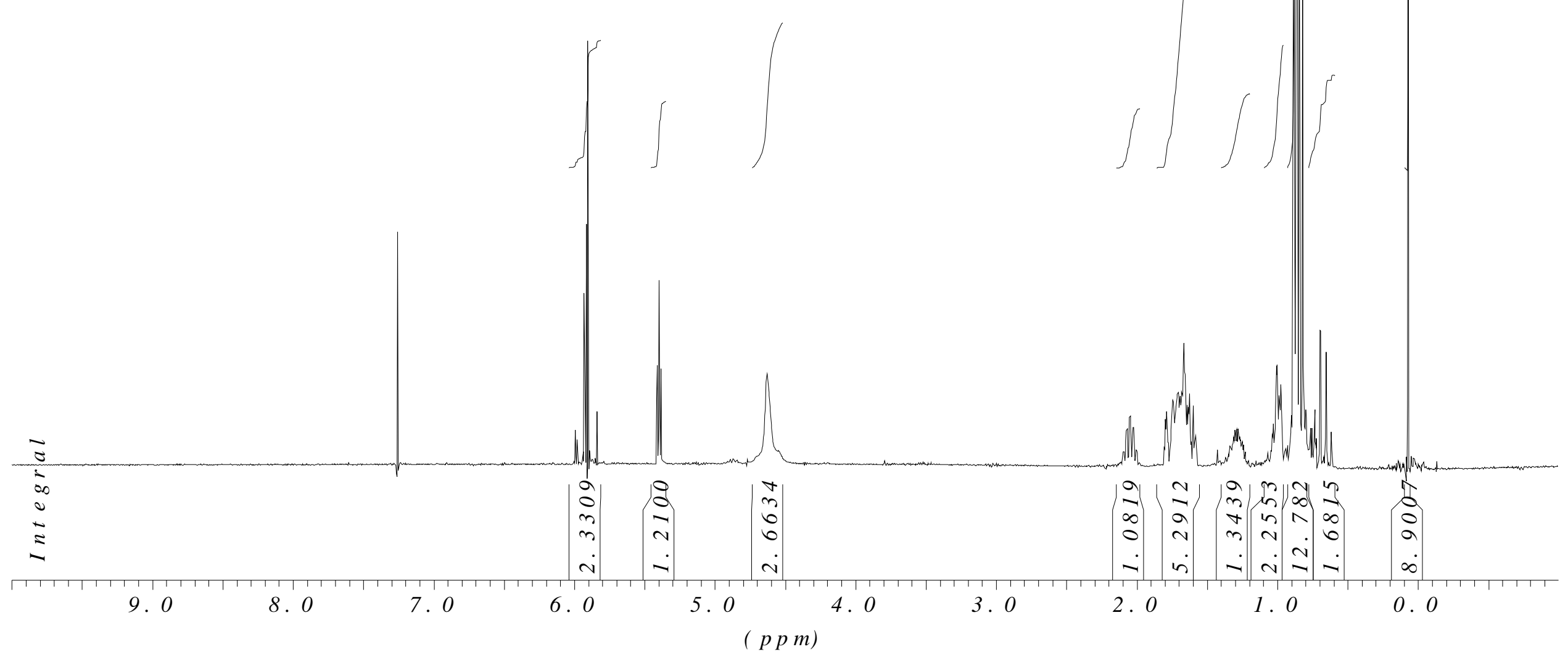




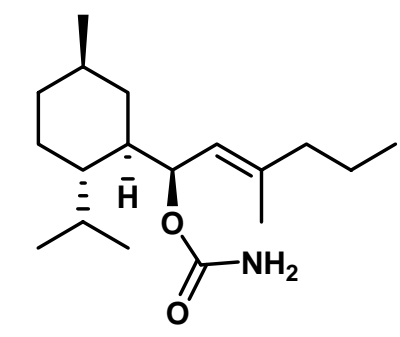

11d

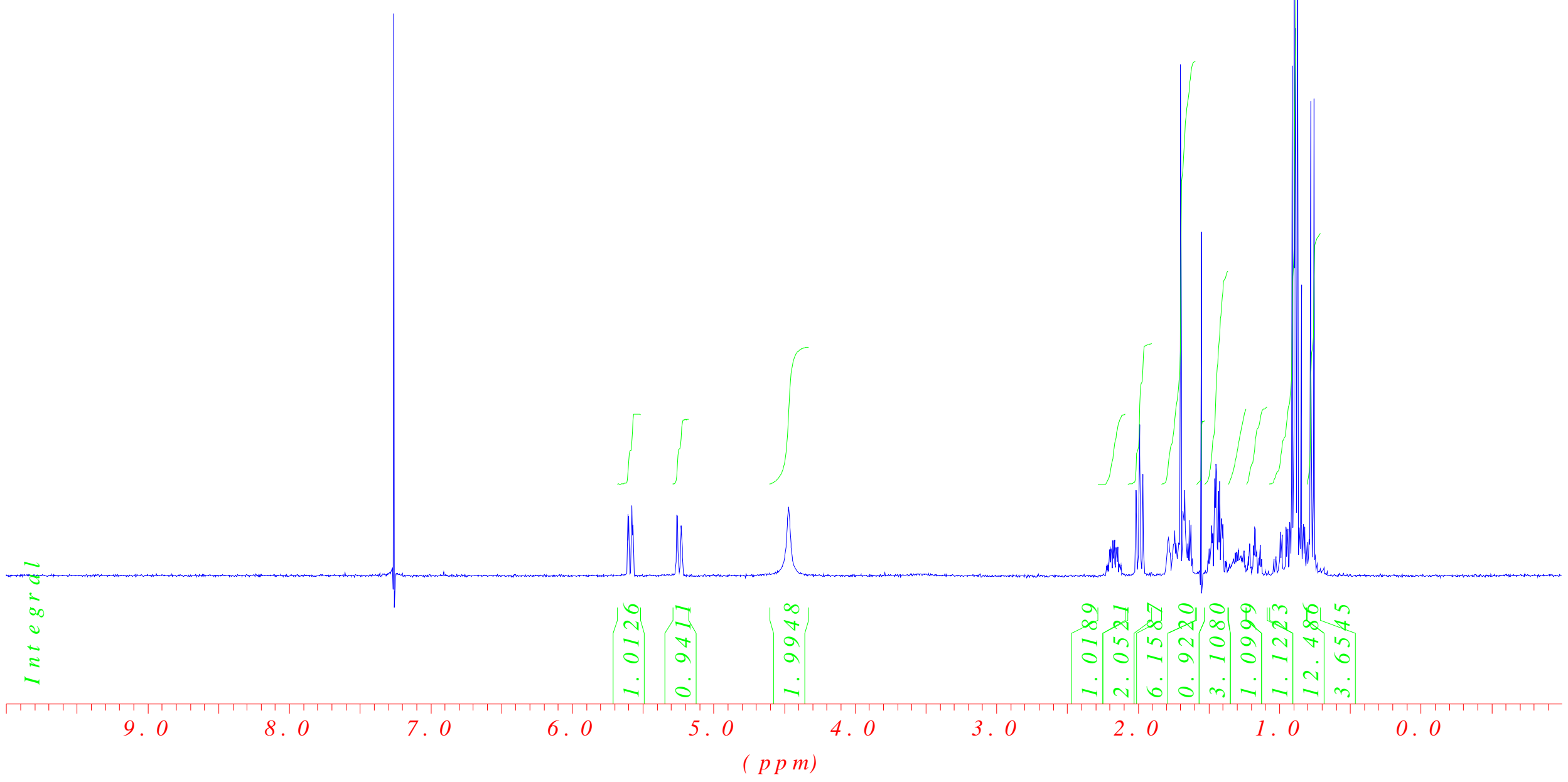




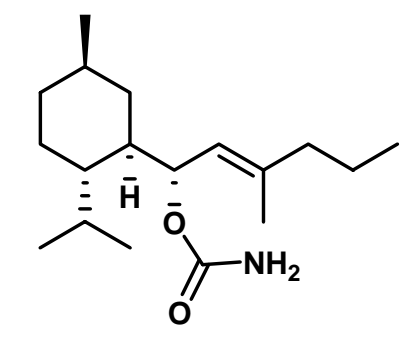

21d

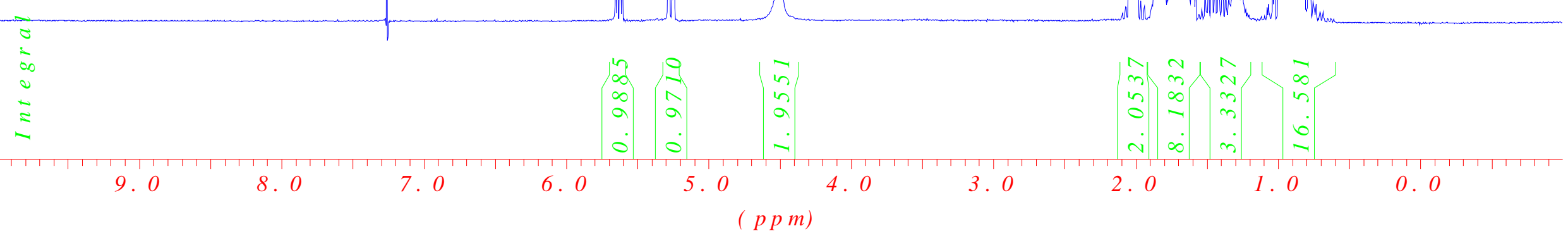




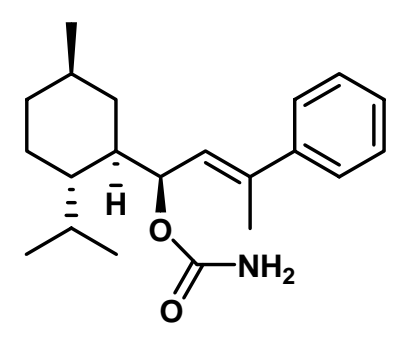

S- 61

$11 e$

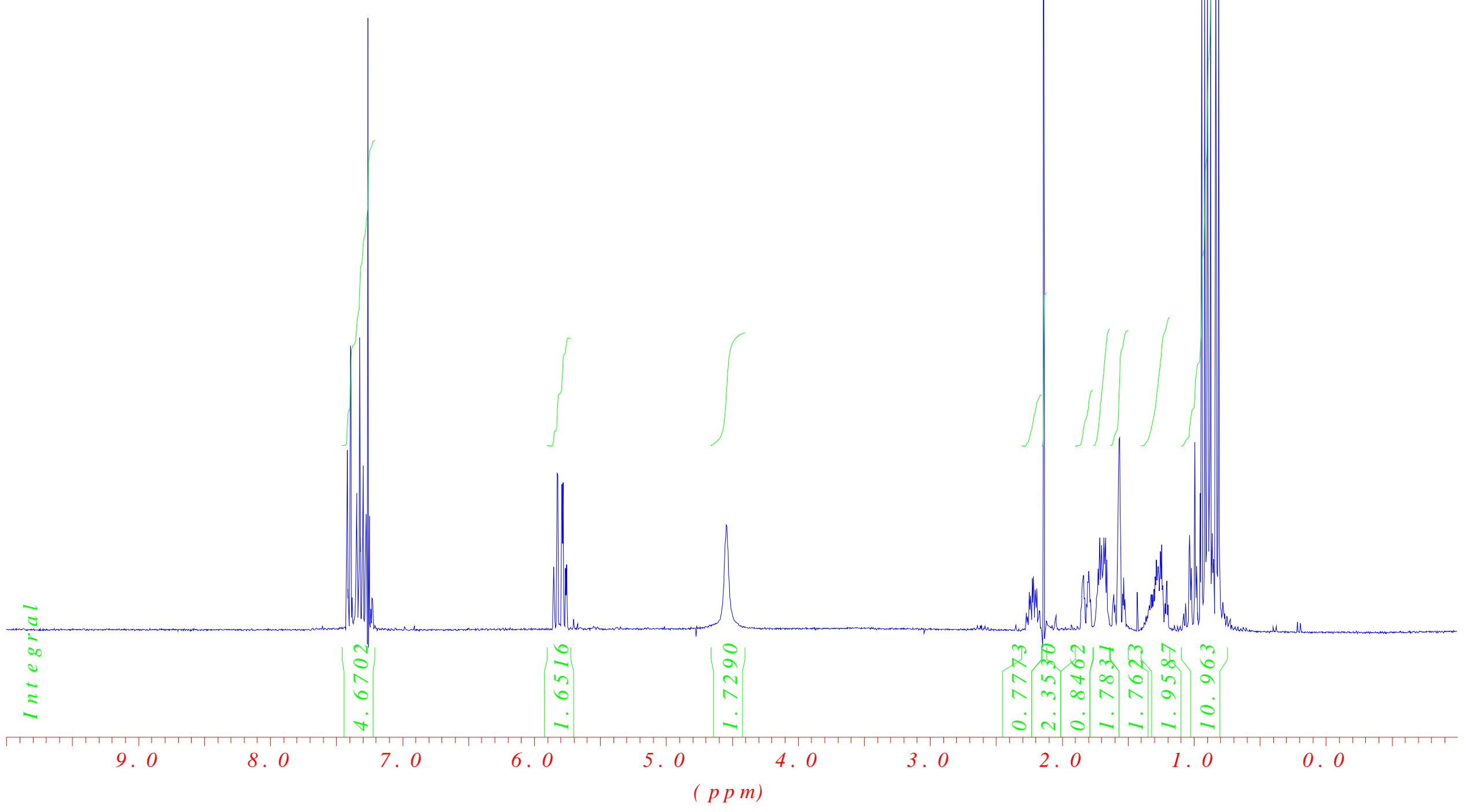




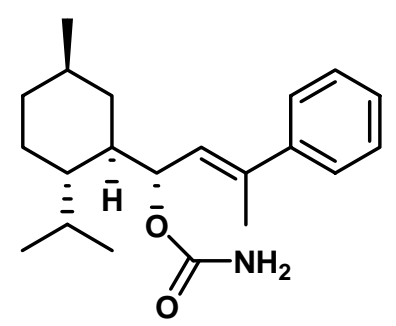

21e

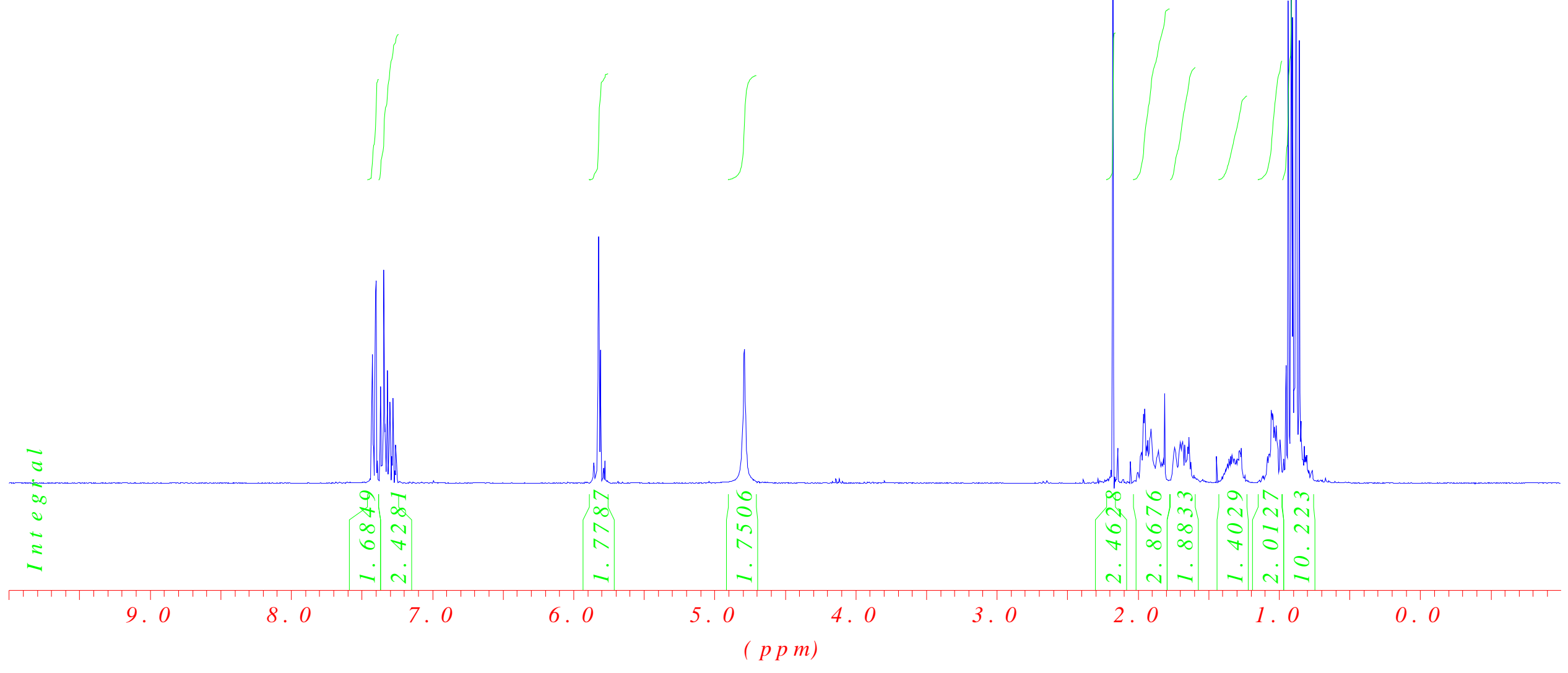




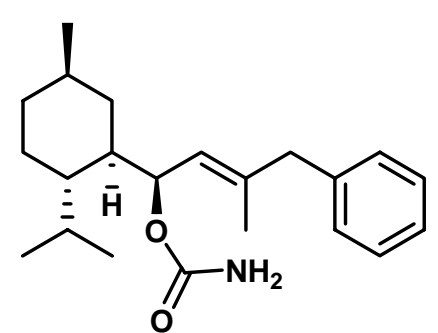

$11 f$

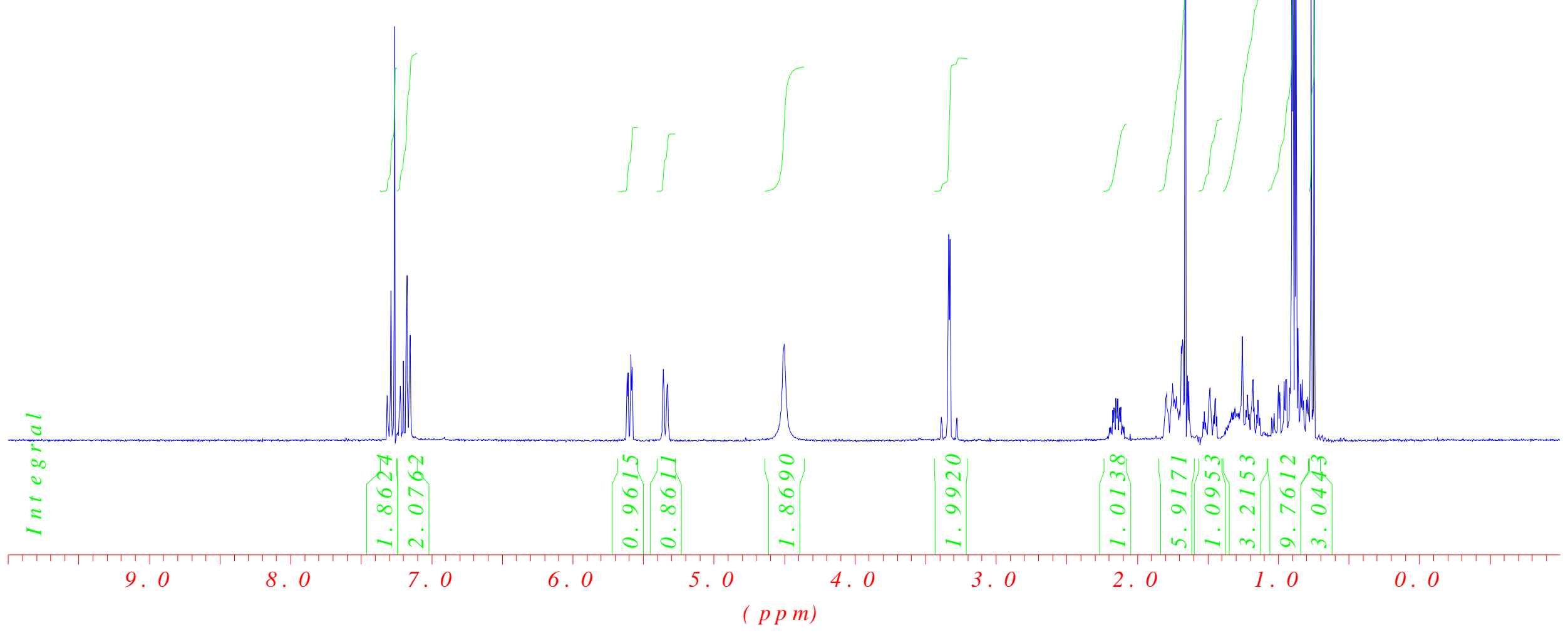




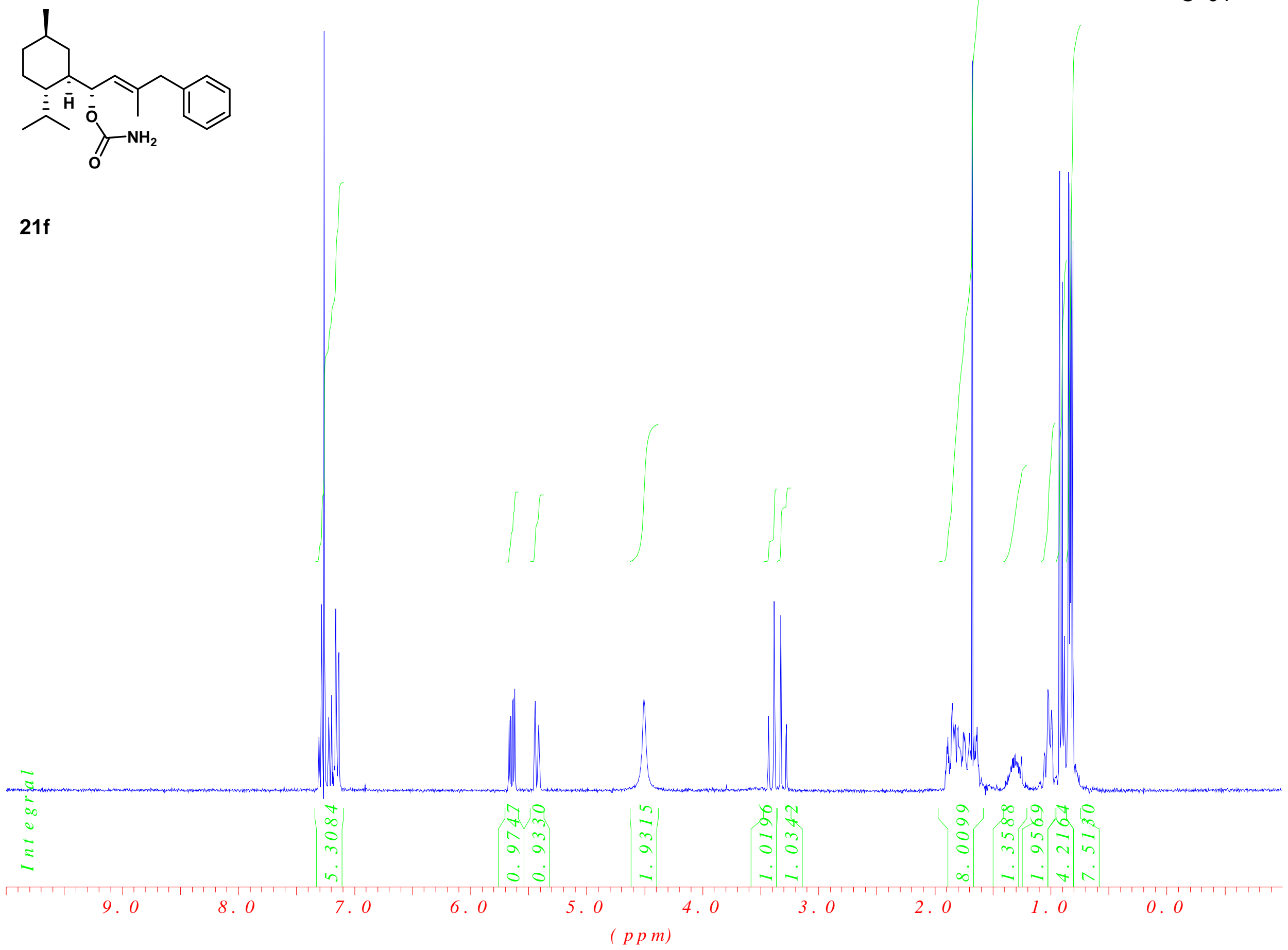




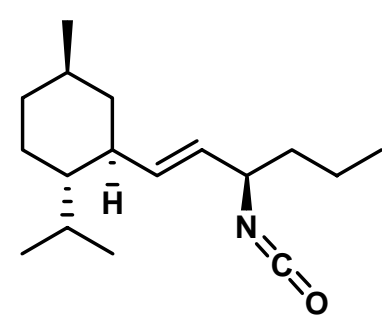

S- 65

$6 a$

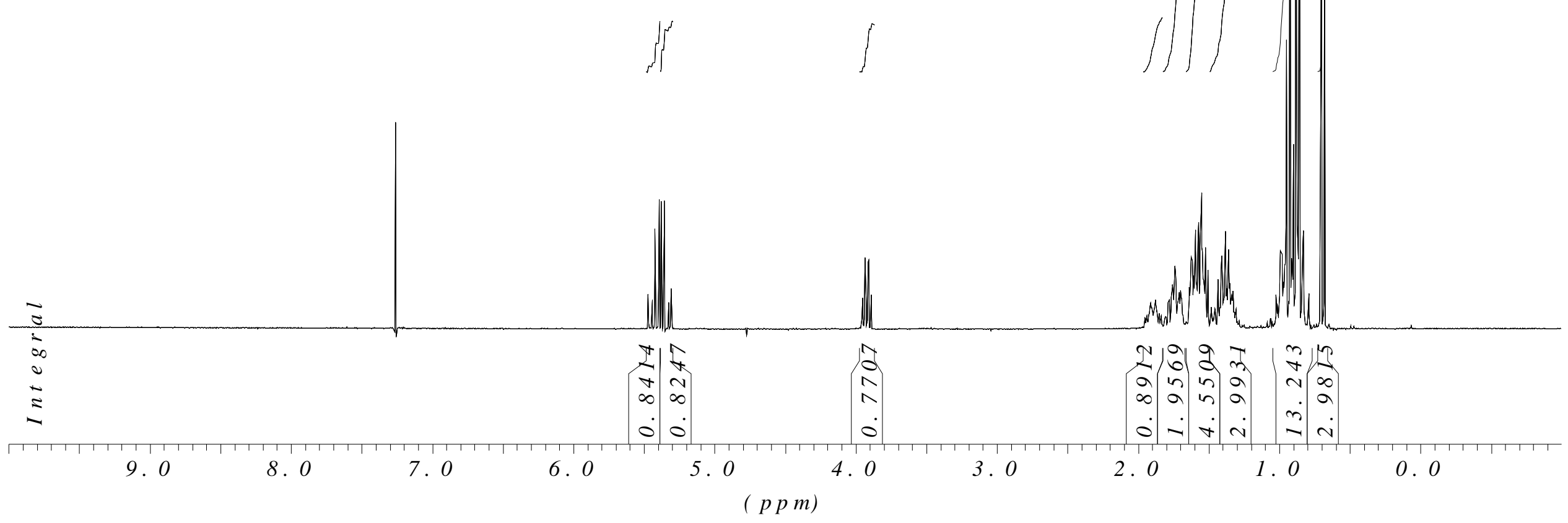




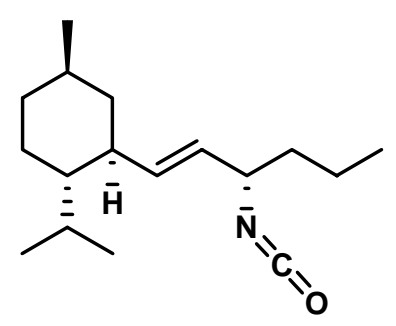

$22 a$

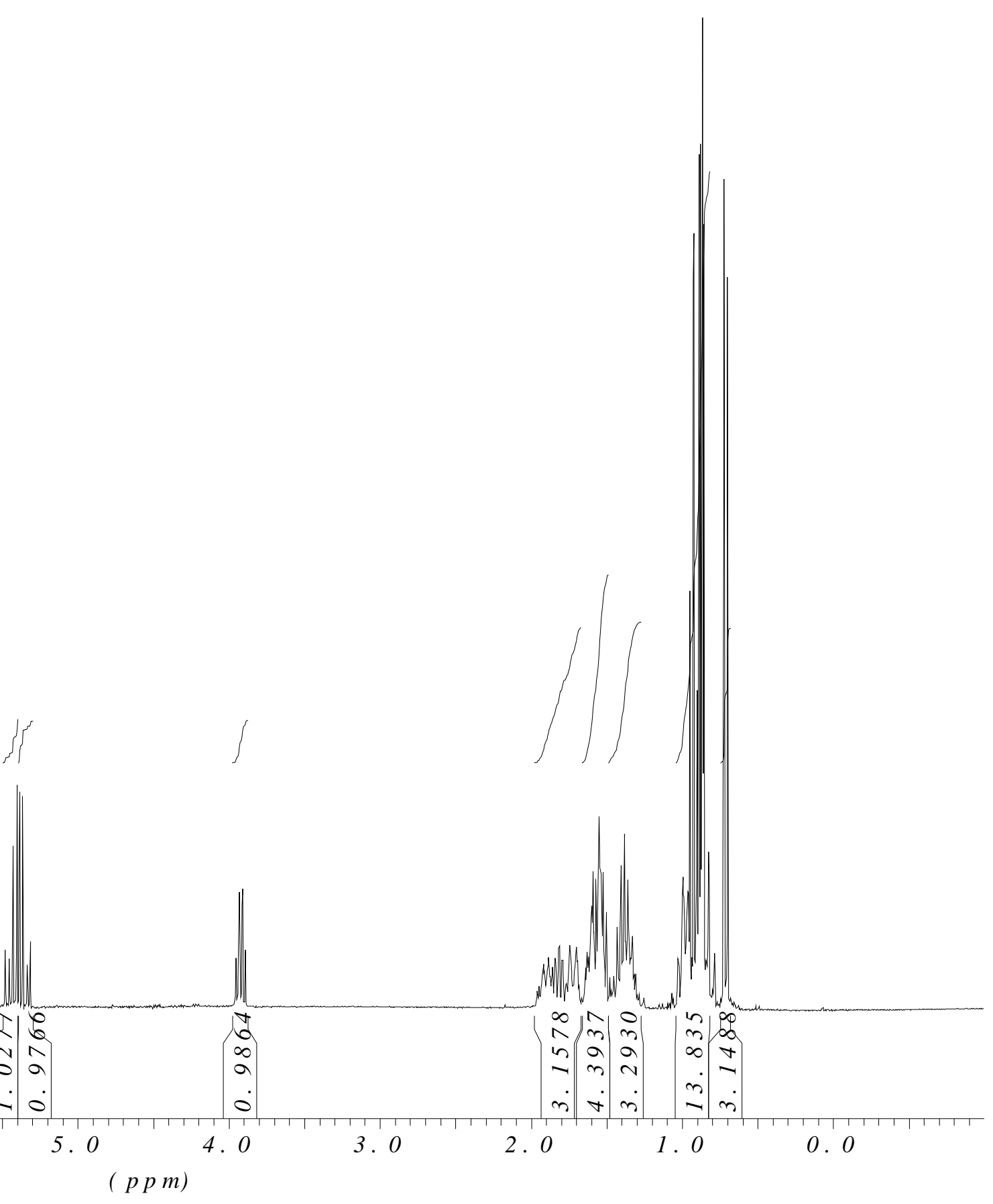




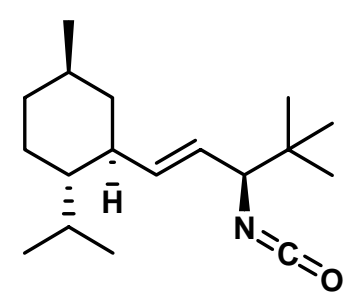

$6 b$

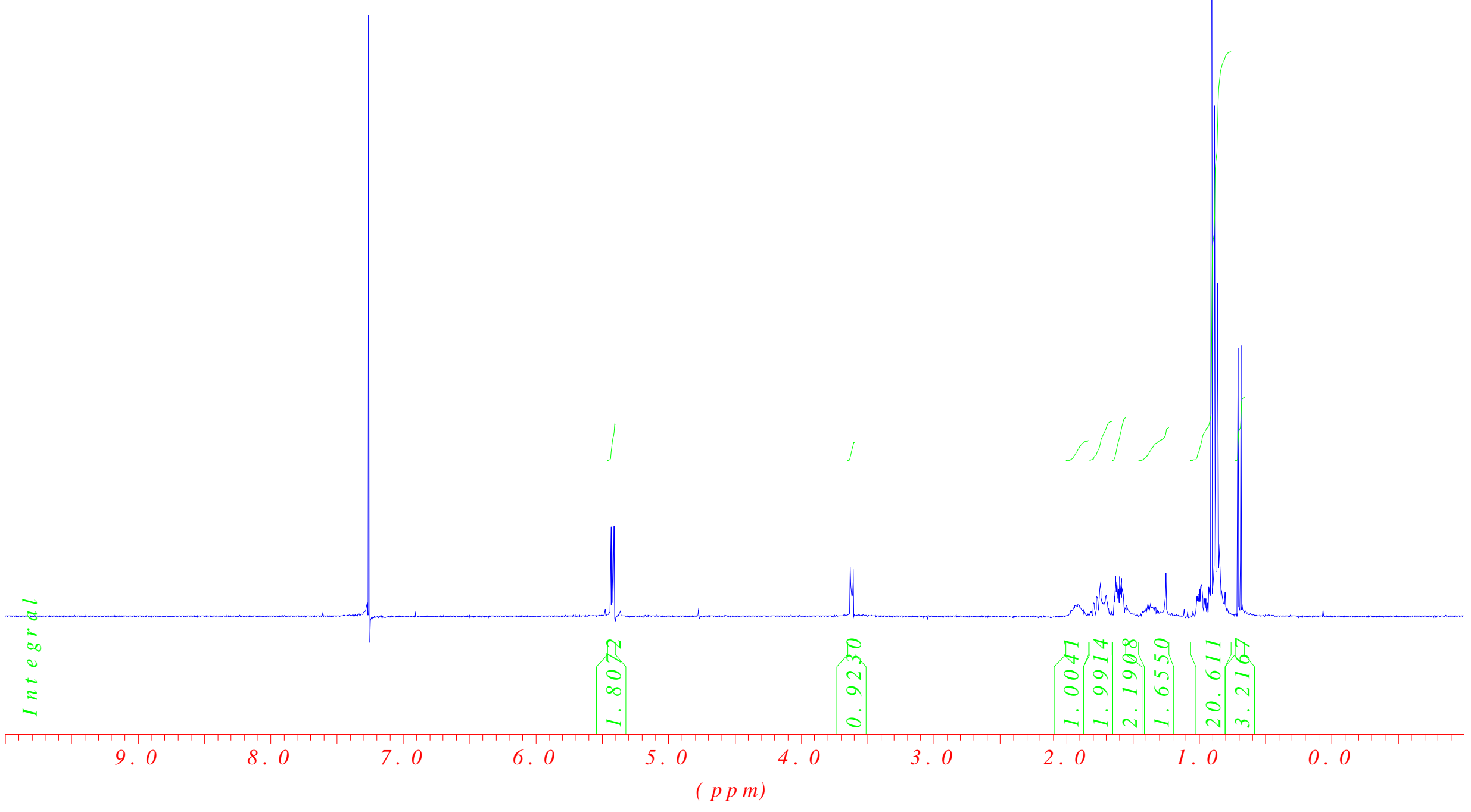




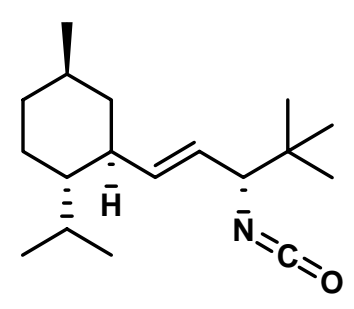

$22 b$

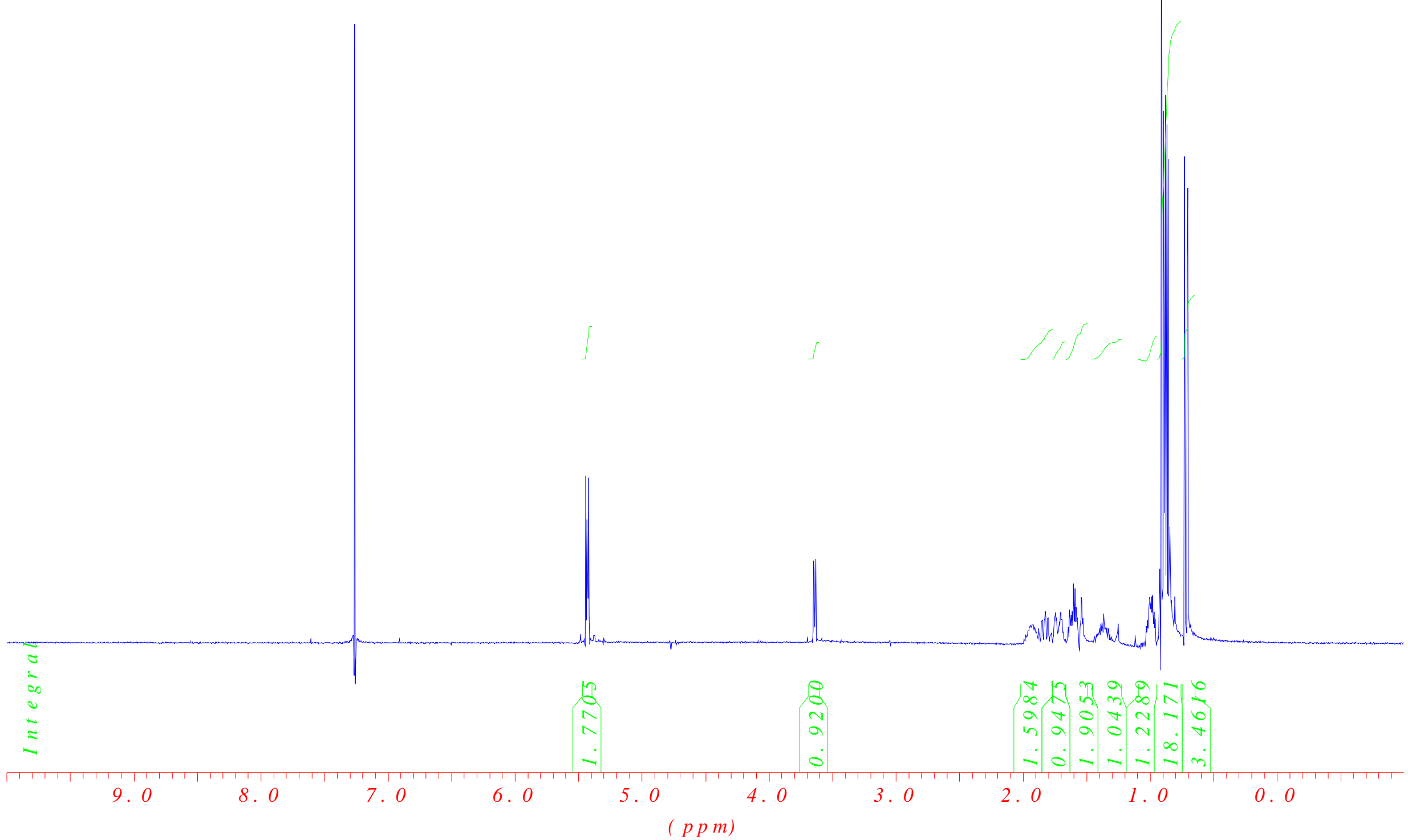




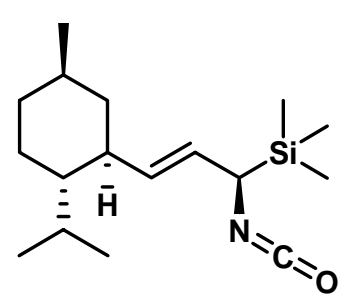

$6 c$

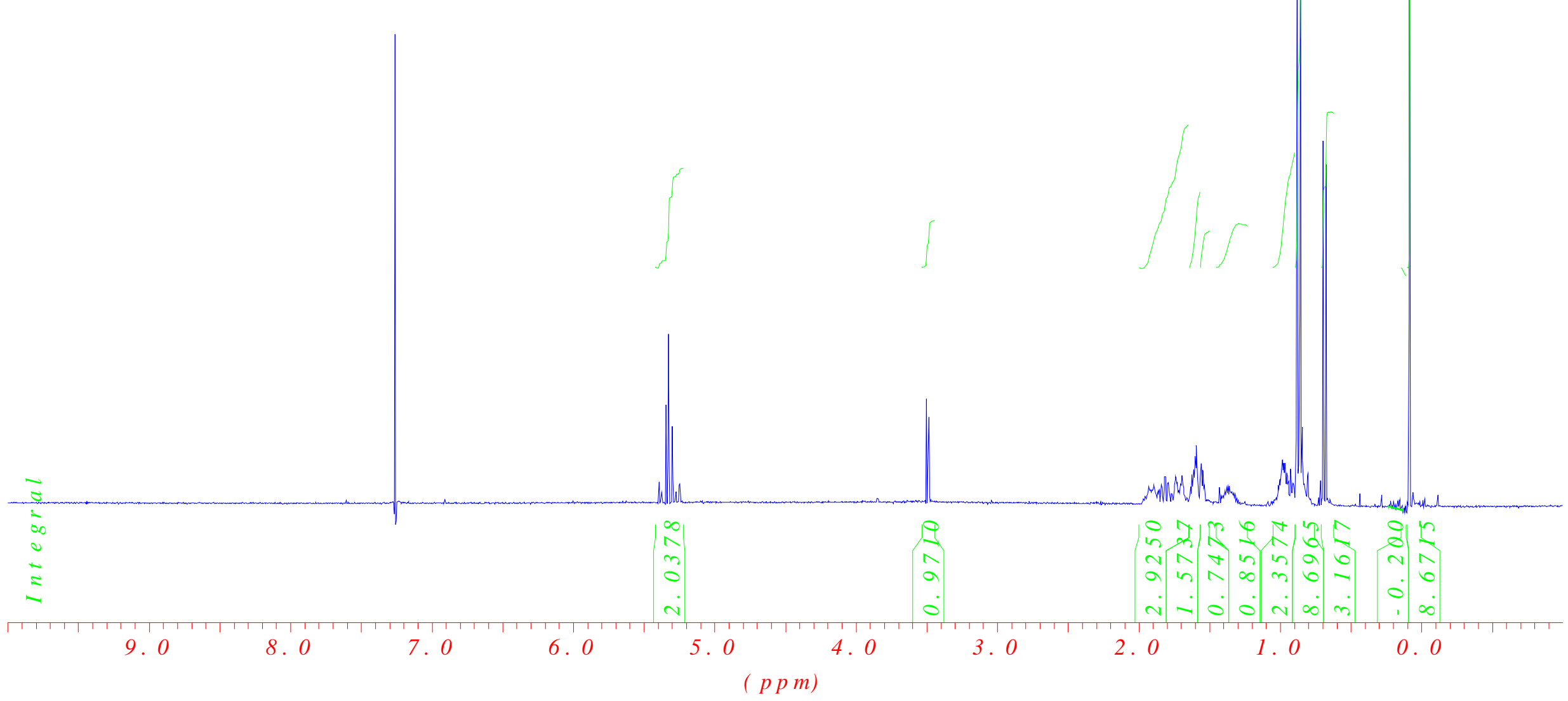




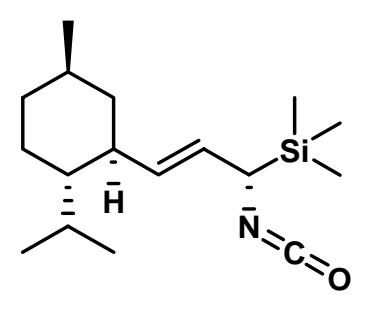

22c

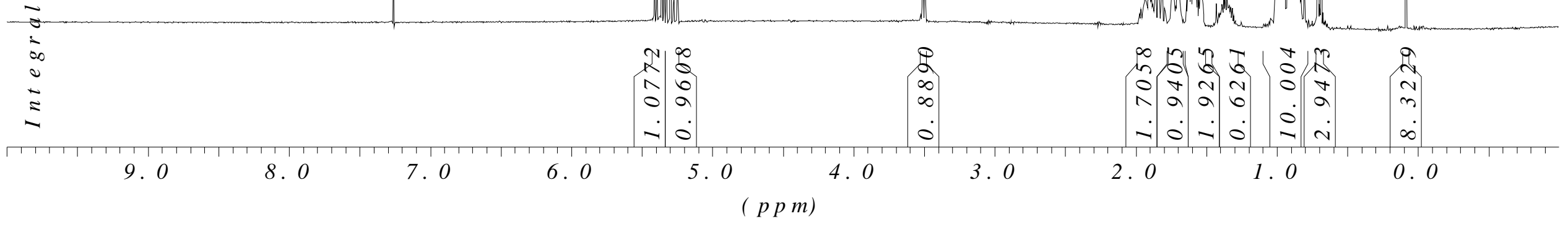




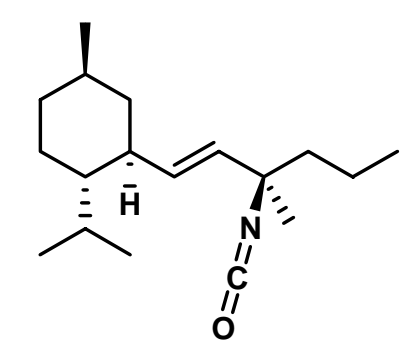

S- 71

6d

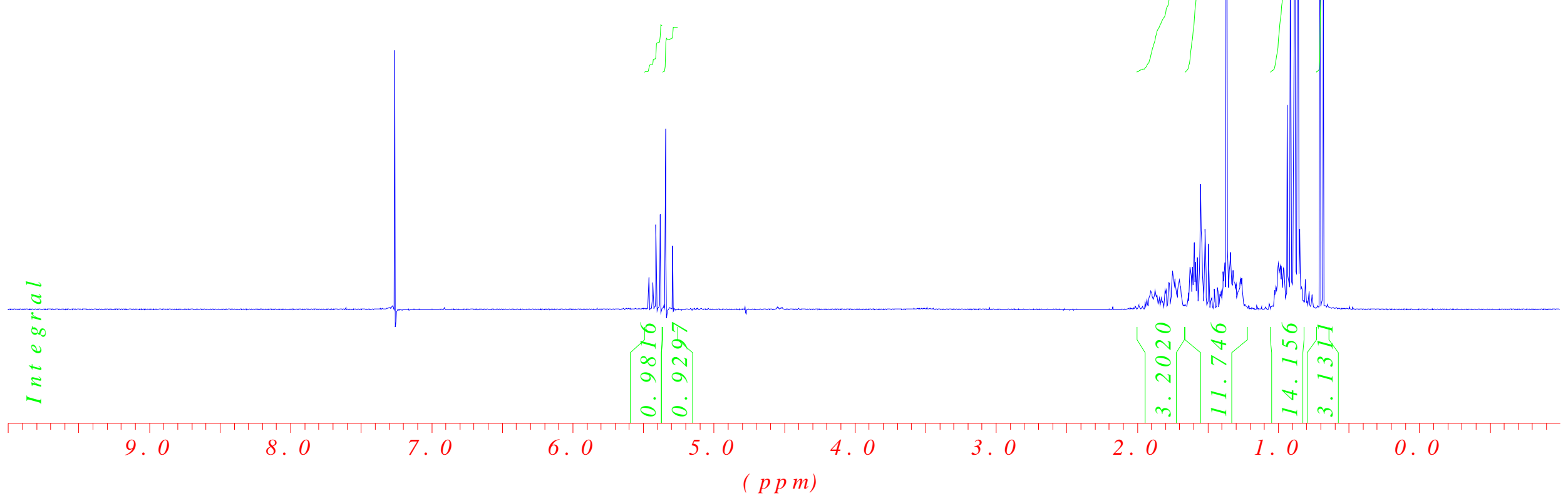




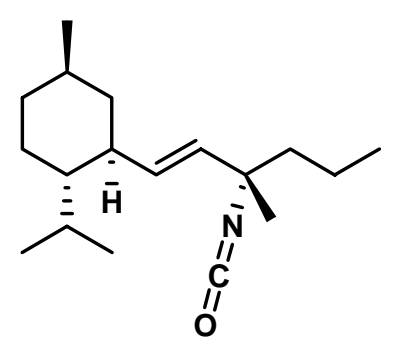

22d

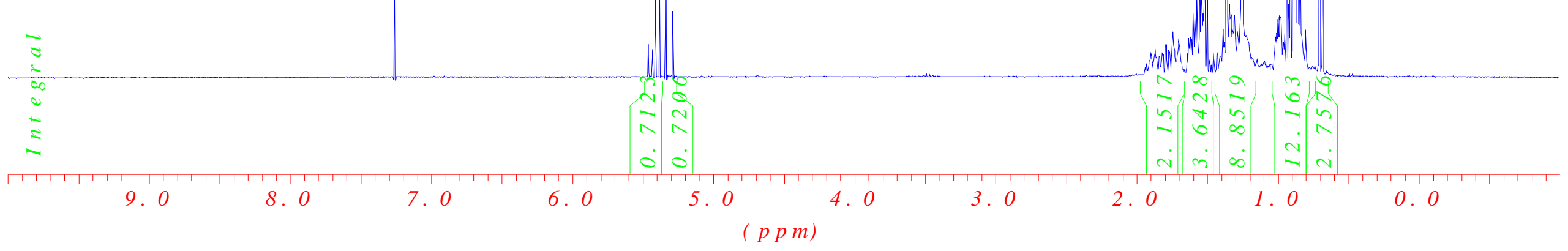




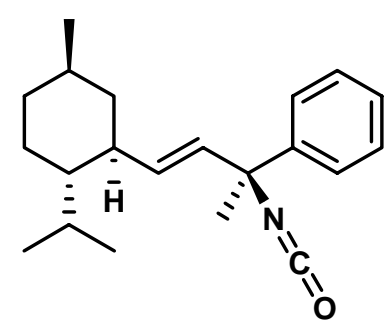

S- 73

$6 e$

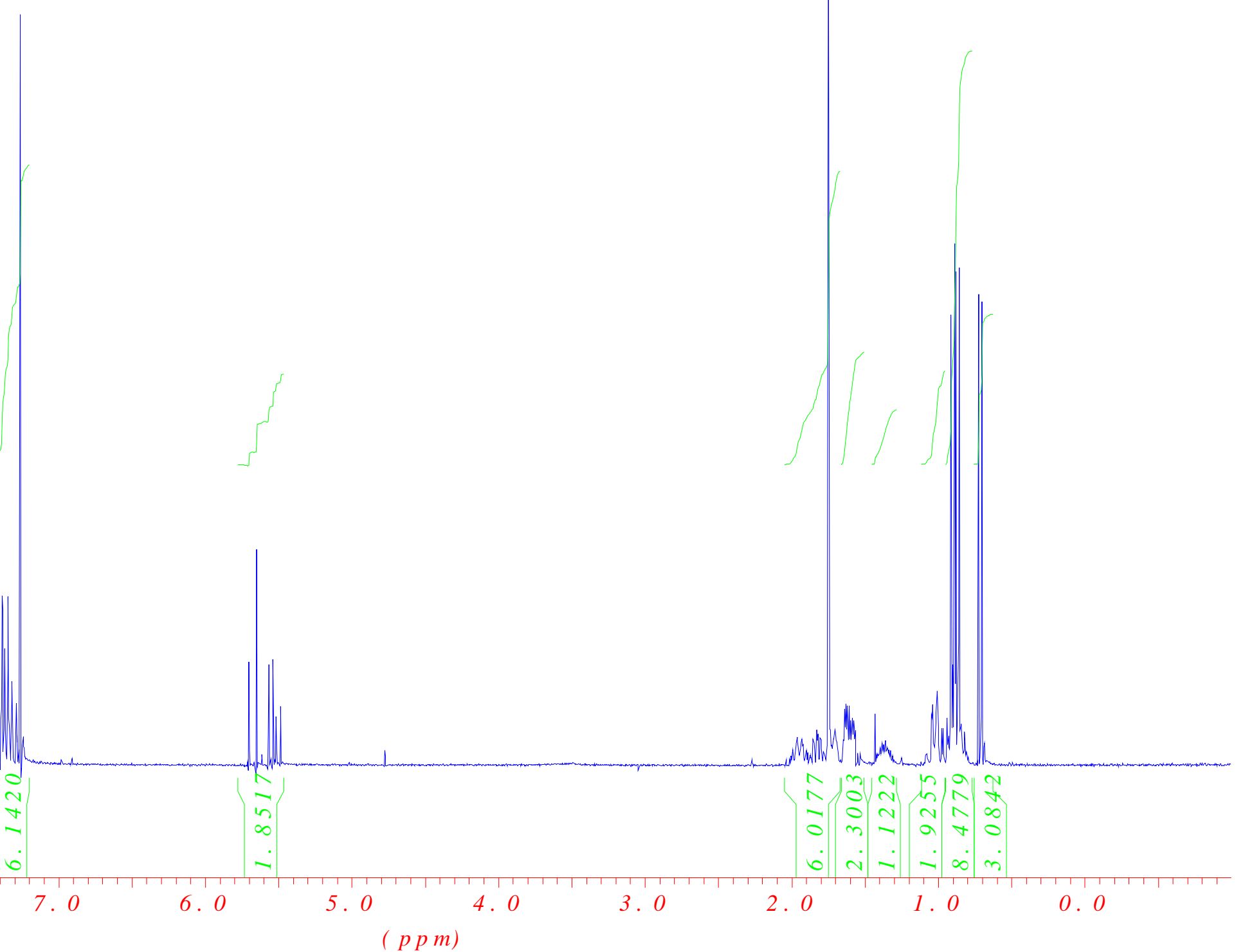




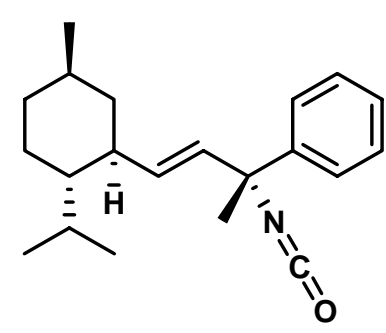

$22 e$

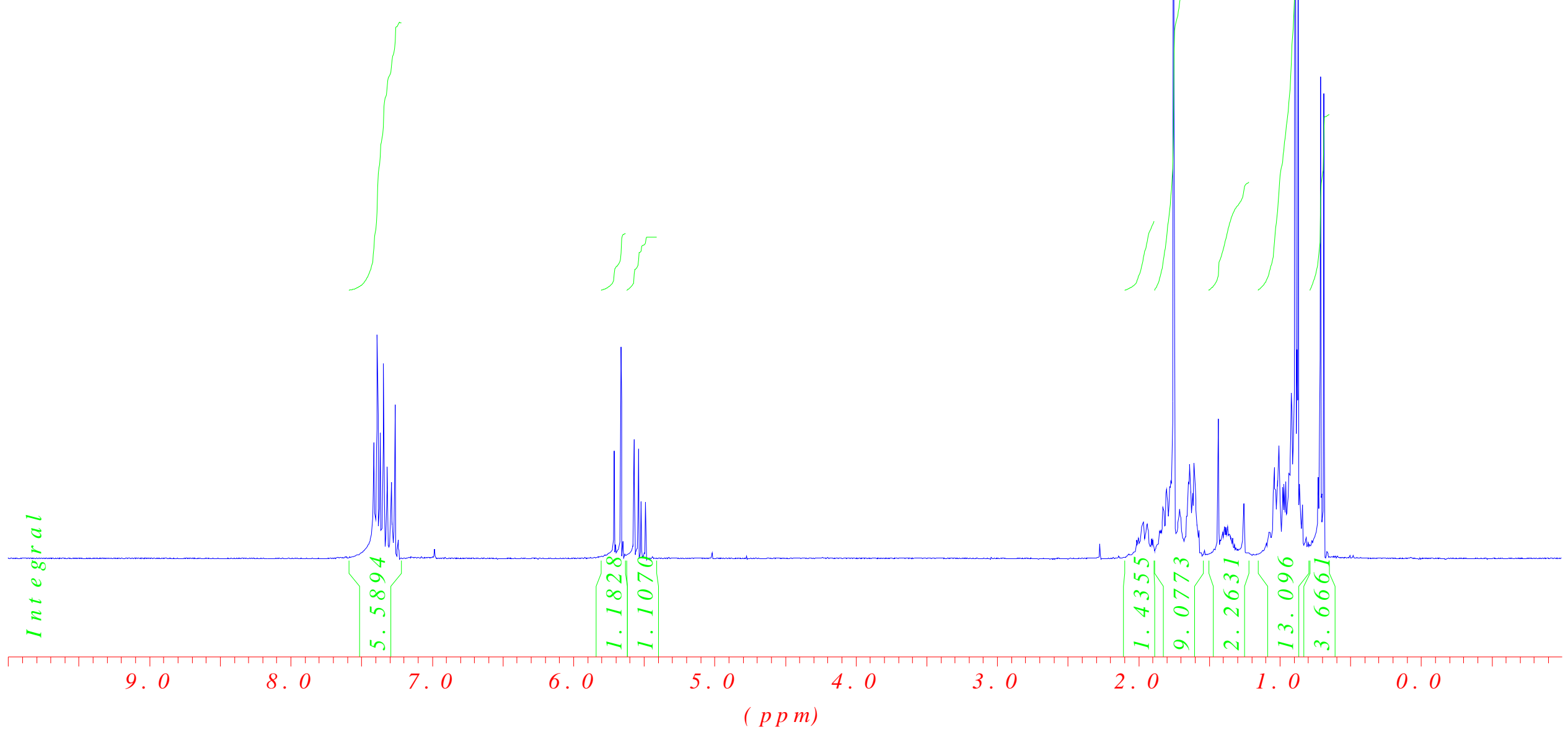




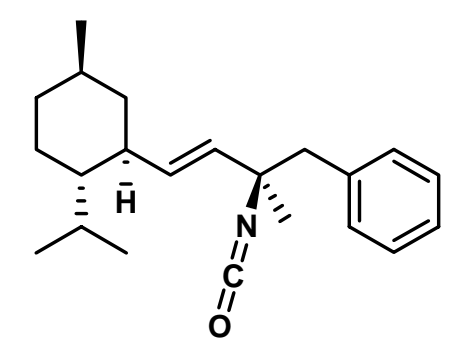

S- 75

$6 f$

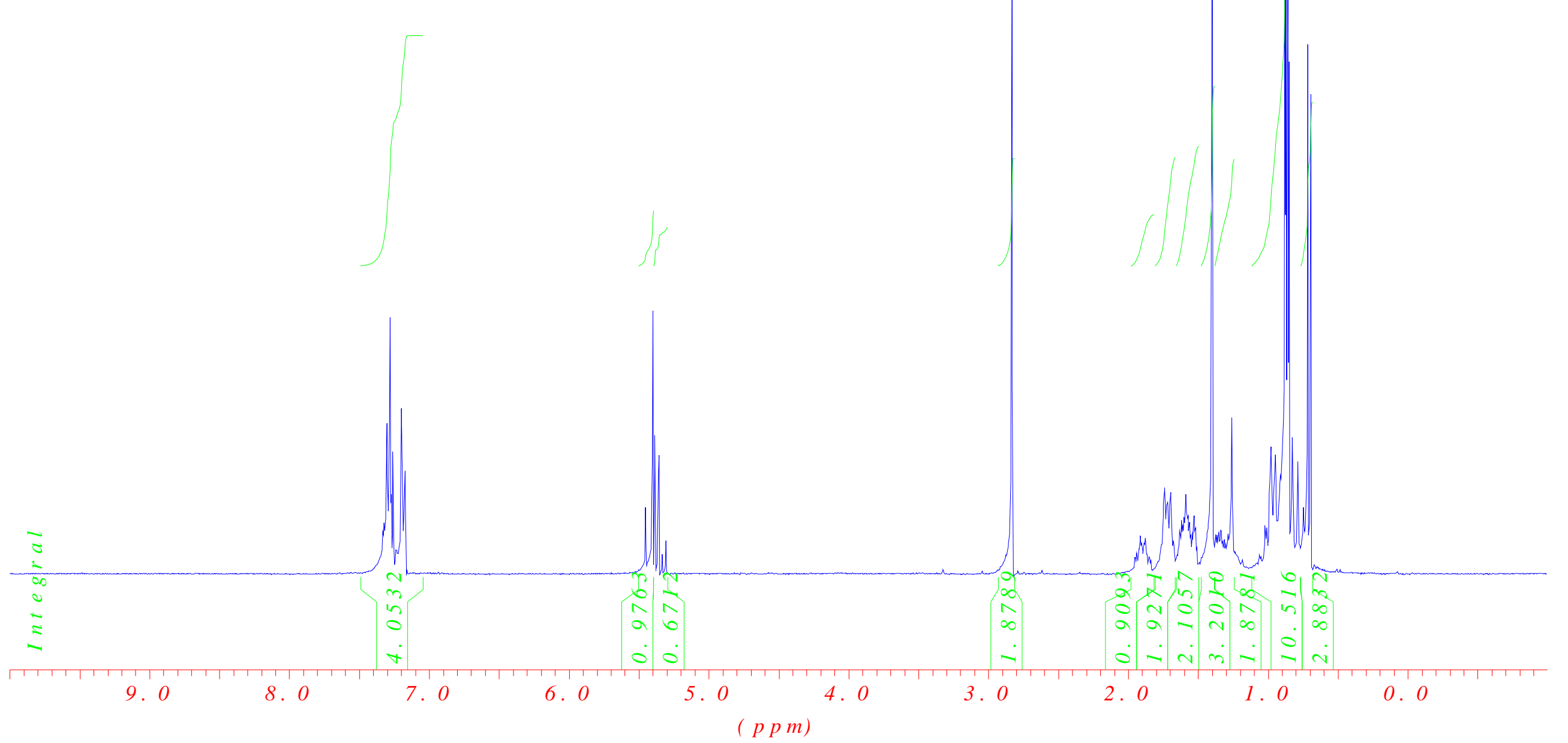




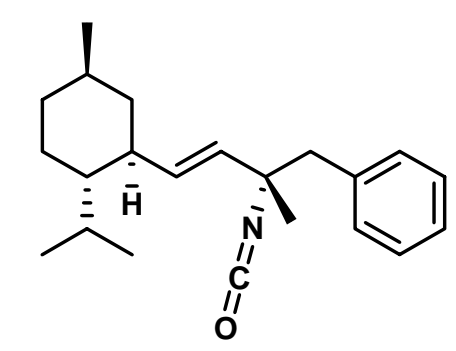

$22 f$

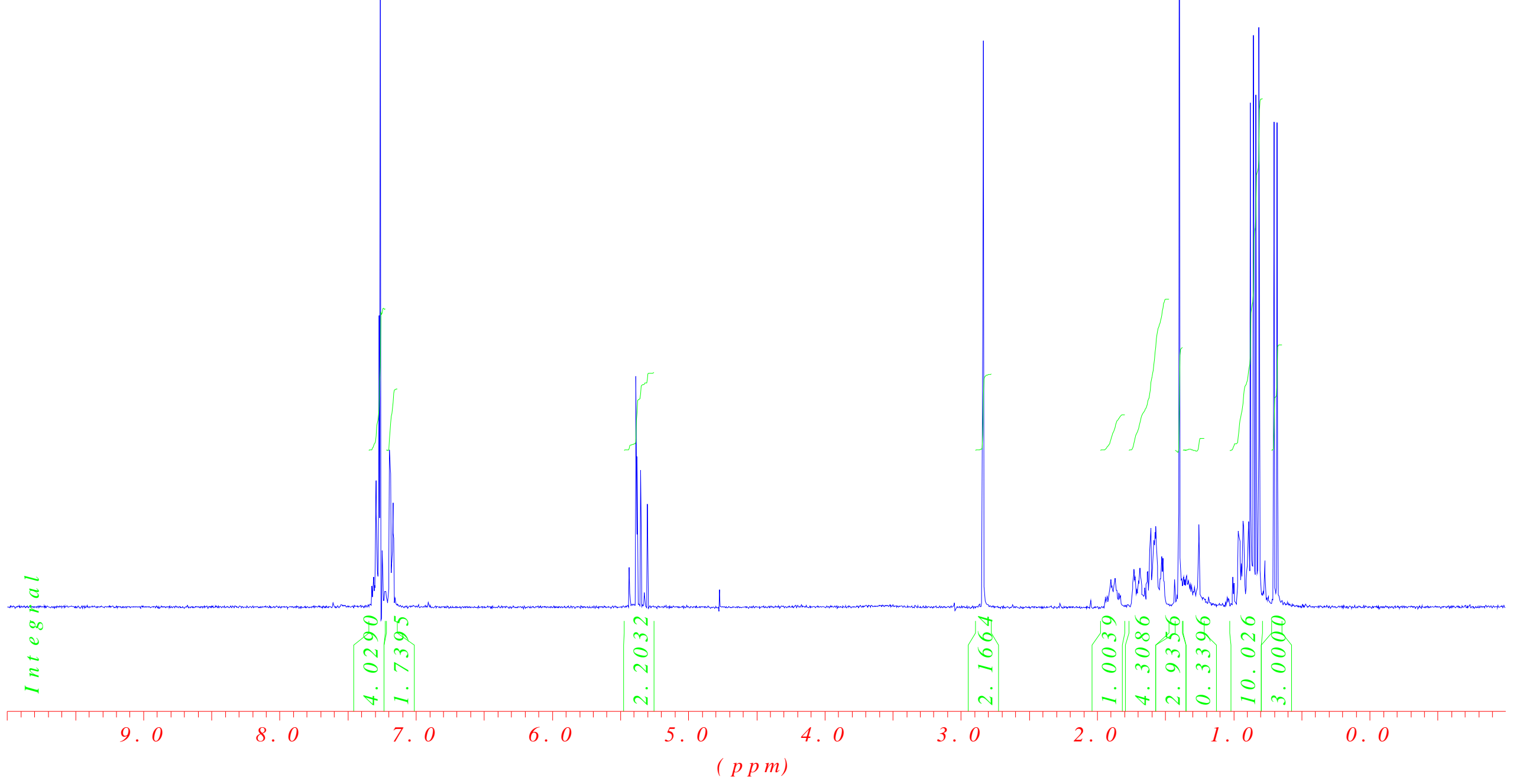




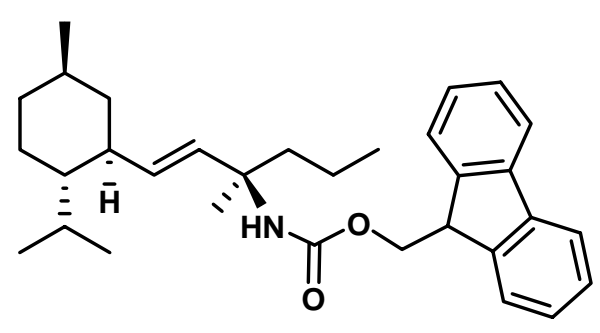

14d

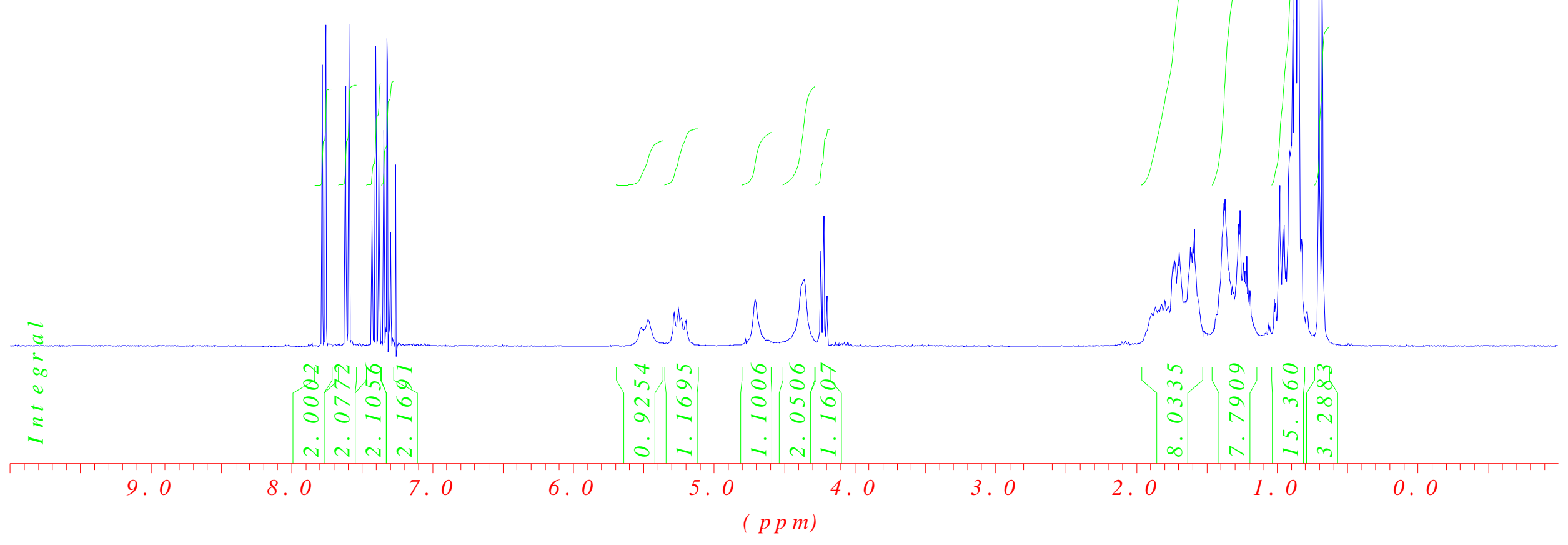




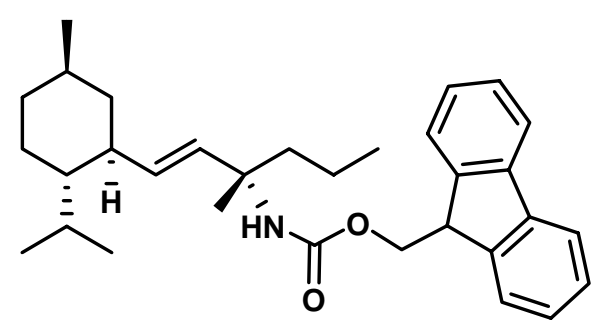

\section{3d}

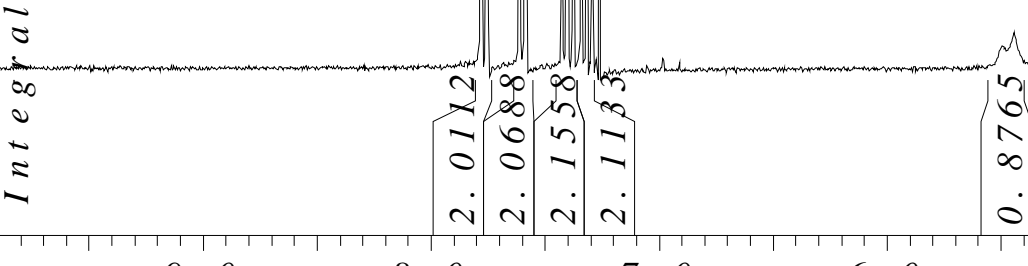

9. 0

8. 0

7. 0

6. 0

$\begin{array}{lllll}1 & 0 & 0 & 0 & 0\end{array}$

$\begin{array}{lllll}1 & 0 & \ddots & m & 0 \\ \infty & 0 & 0 & \ddots\end{array}$

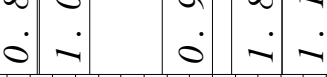

( $p p m)$

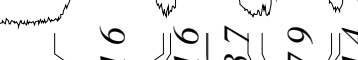

$\begin{array}{lllll}-1 & n & n & N \\ 0 & 0 & m & n & n\end{array}$

$\begin{array}{rrrrr}0 & \dot{\gamma} & \dot{r} & \dot{r} & \dot{m}\end{array}$

2. 0

1. 0

0.0 


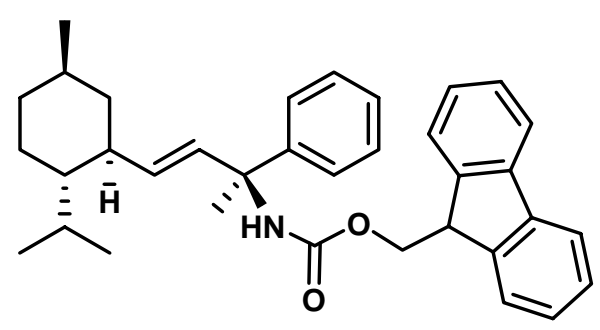

$14 \mathrm{e}$

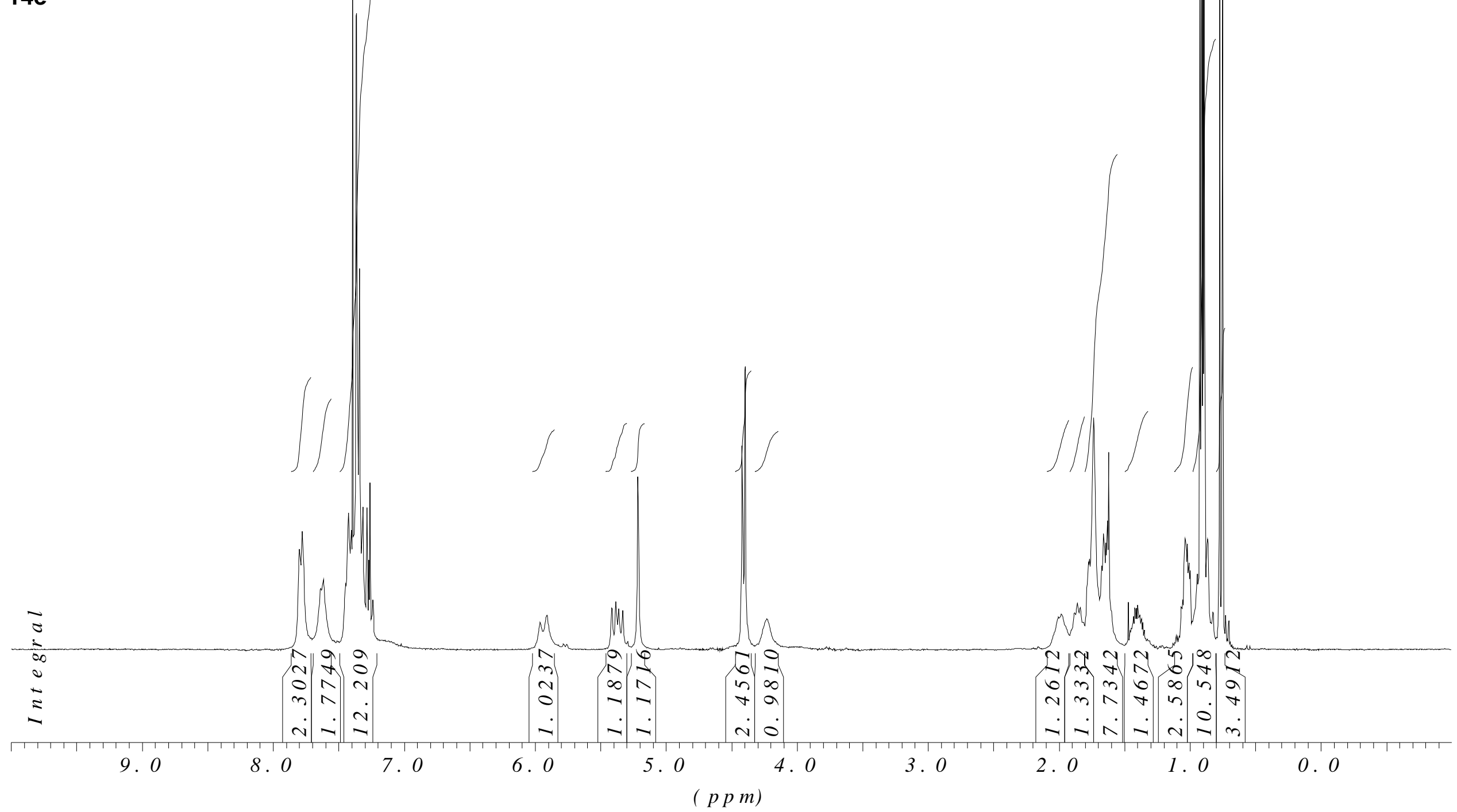




\section{$23 e$}

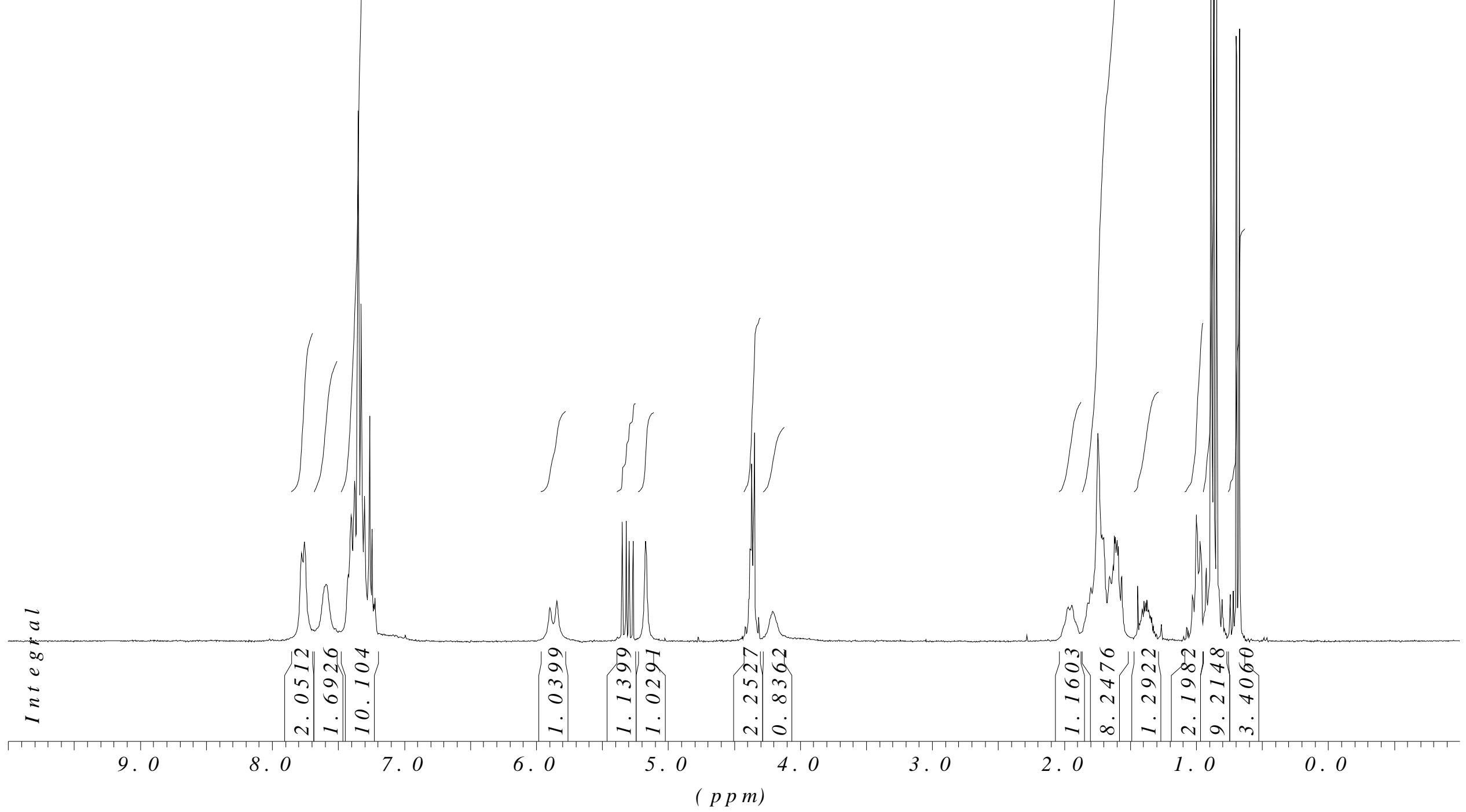




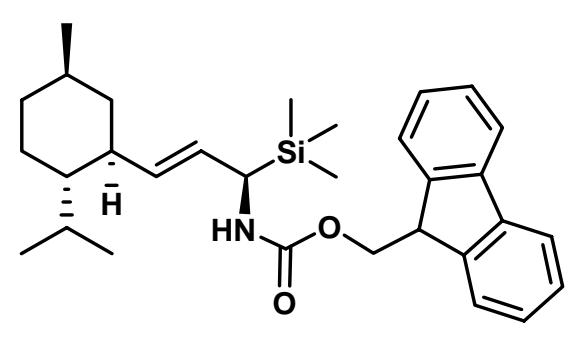

$14 c$

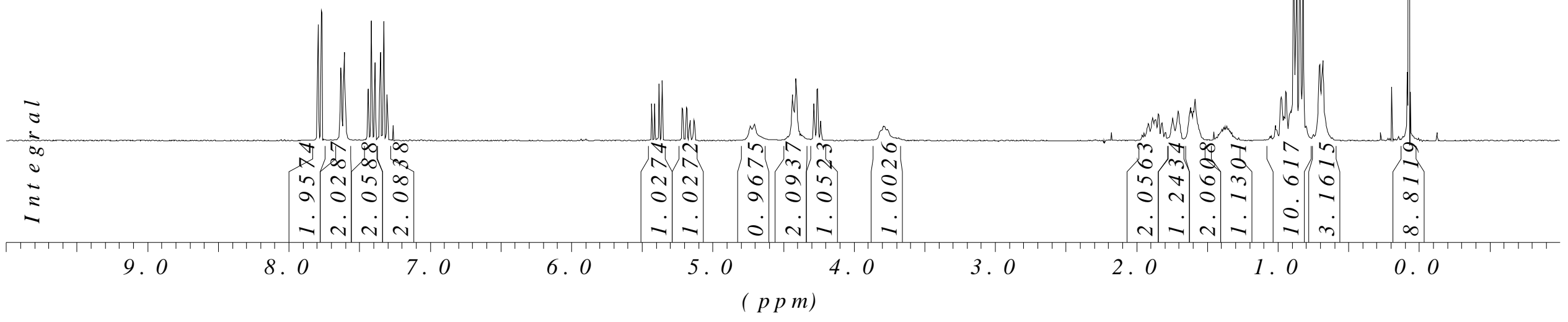




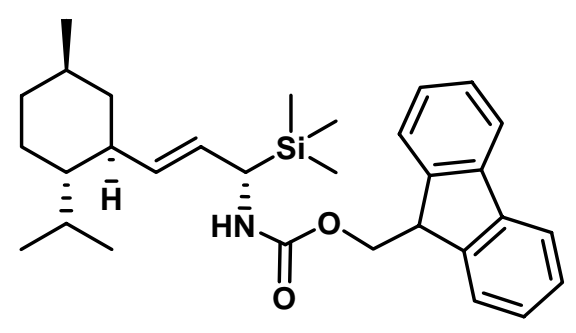

23c

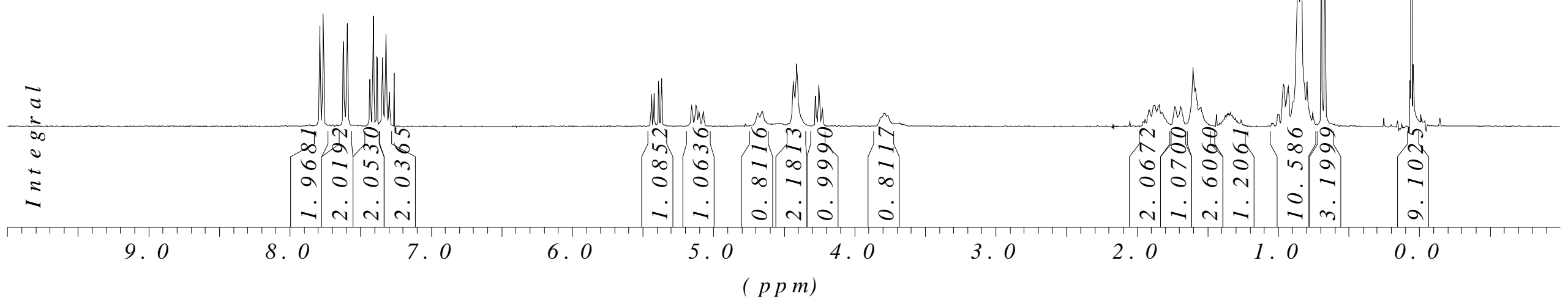




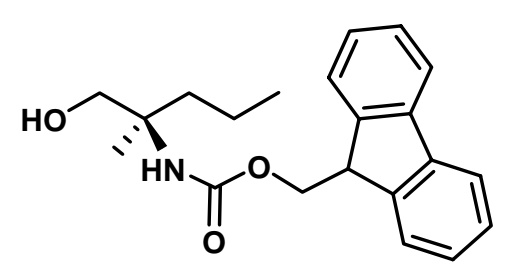

16d

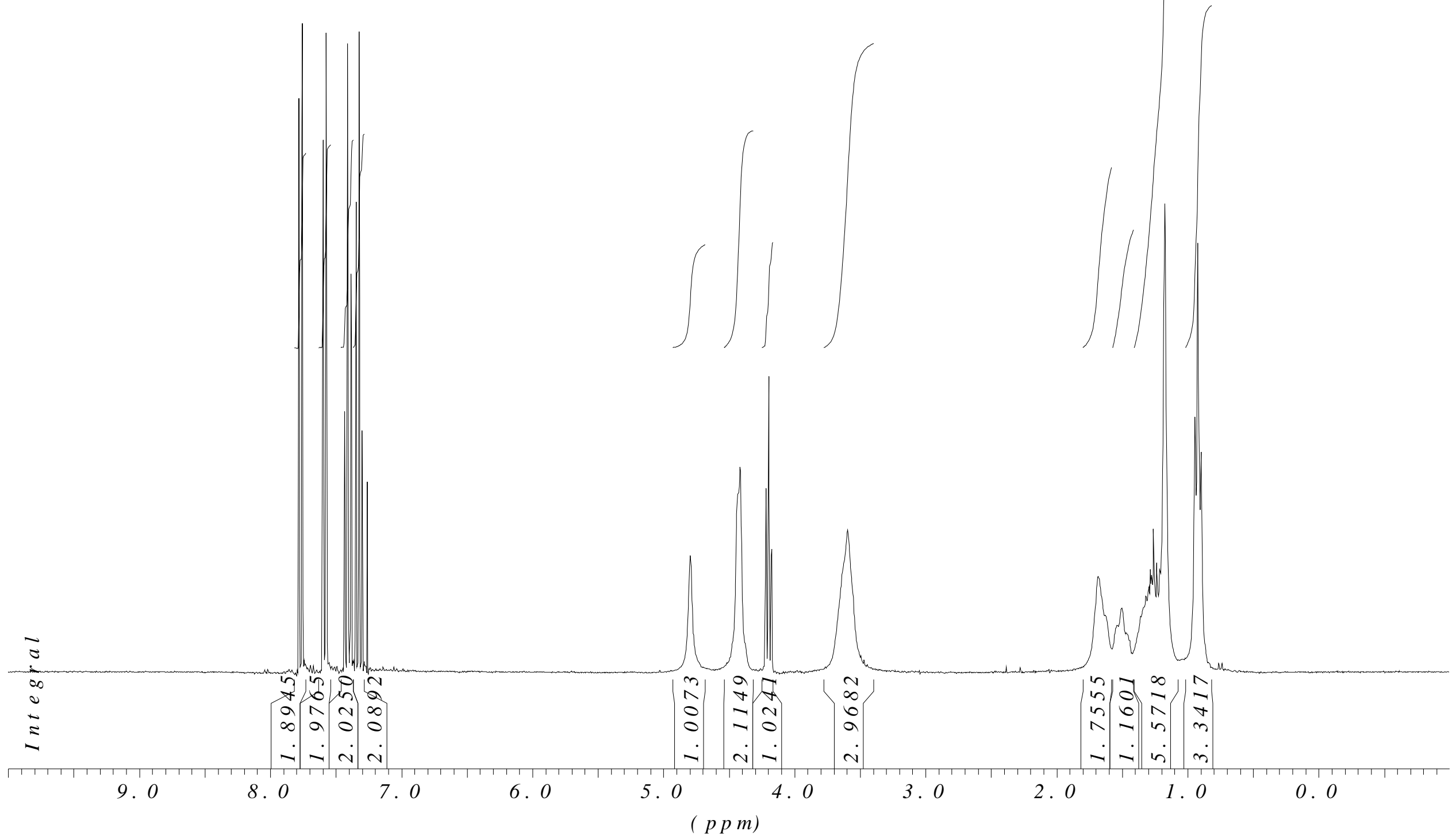




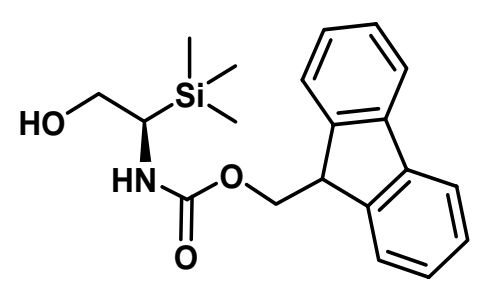

16c

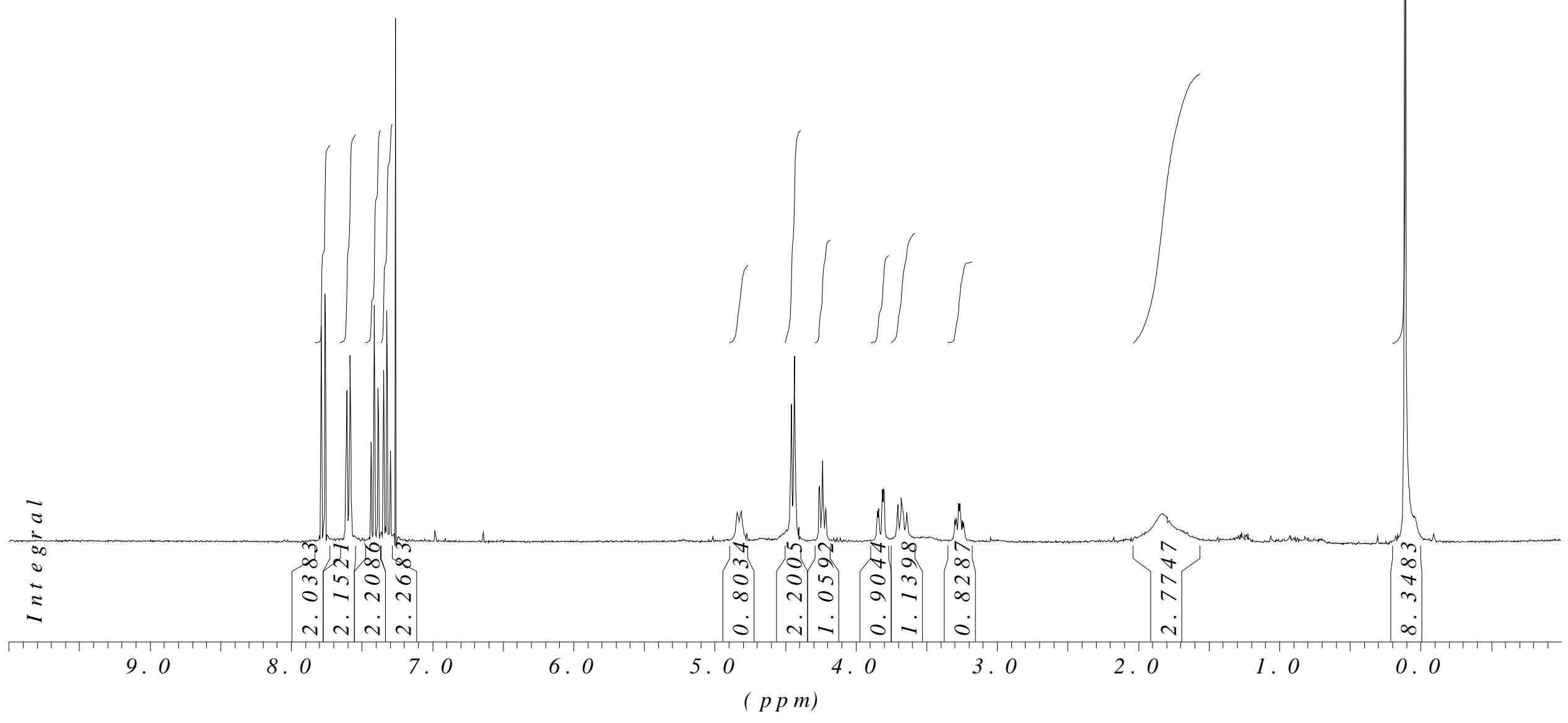




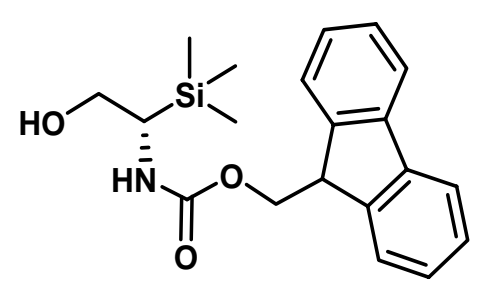

\section{$25 c$}

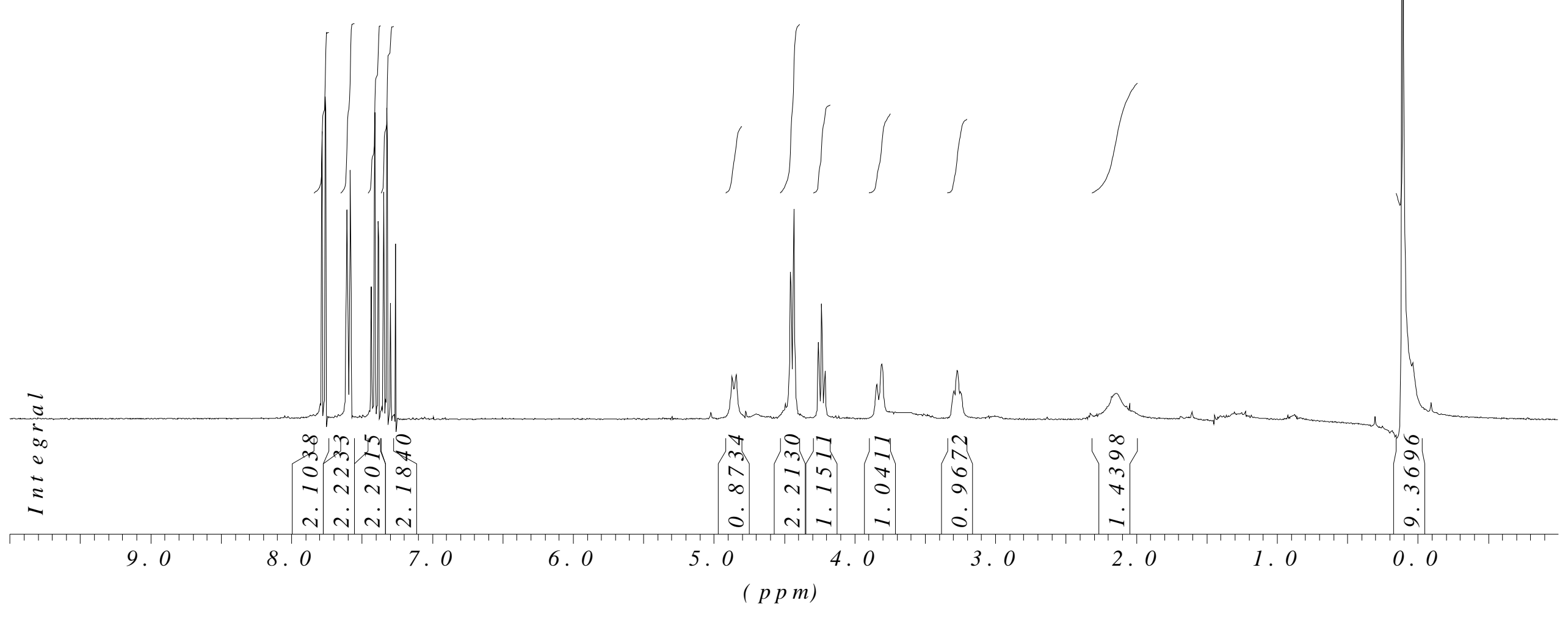




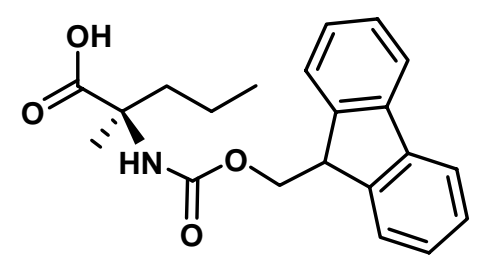

15d

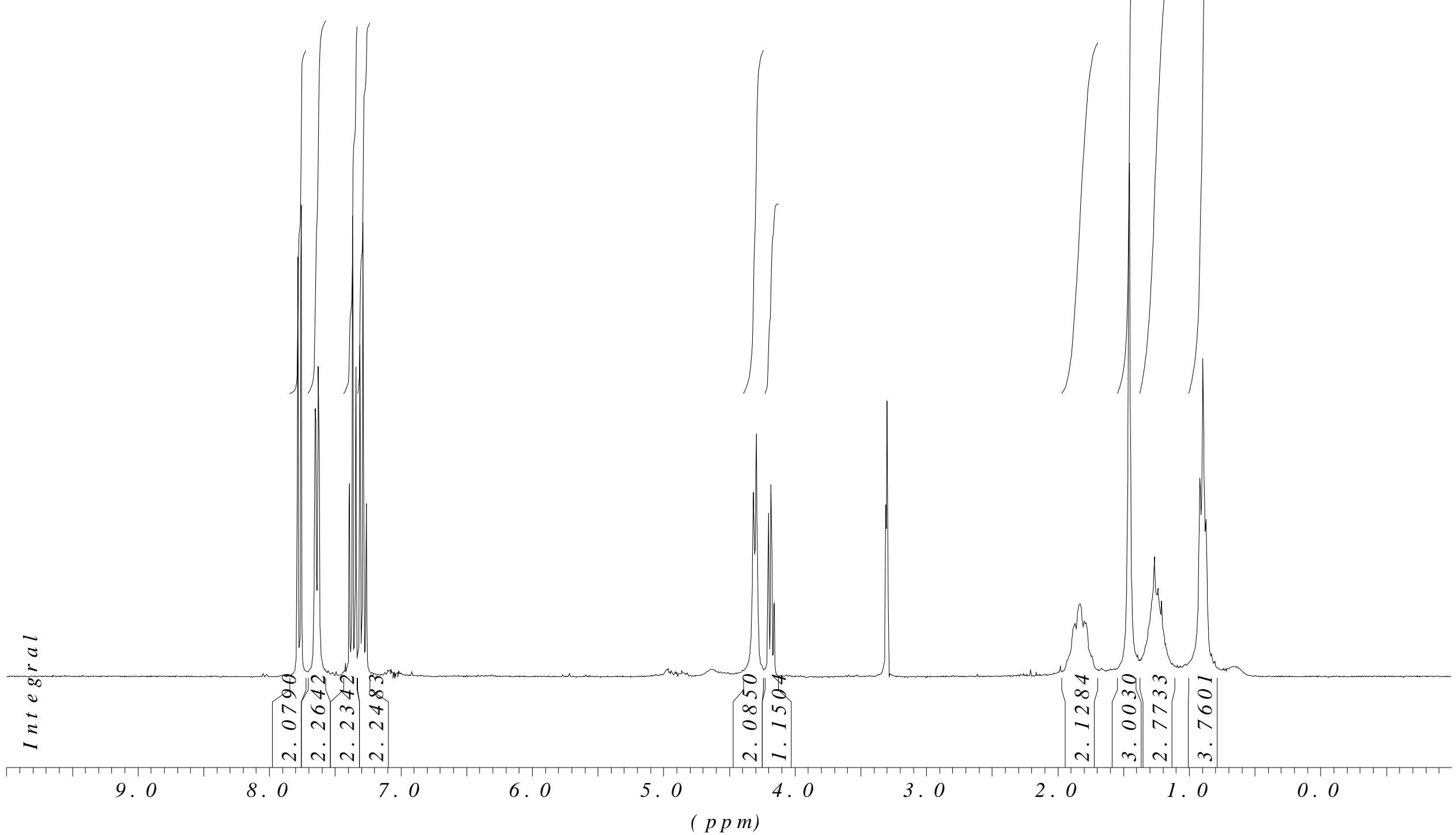




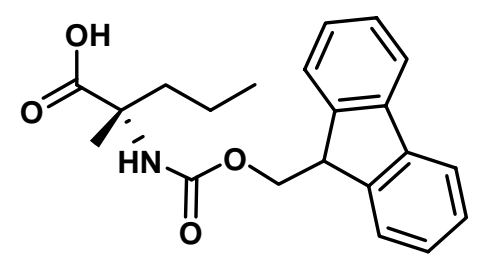

24d

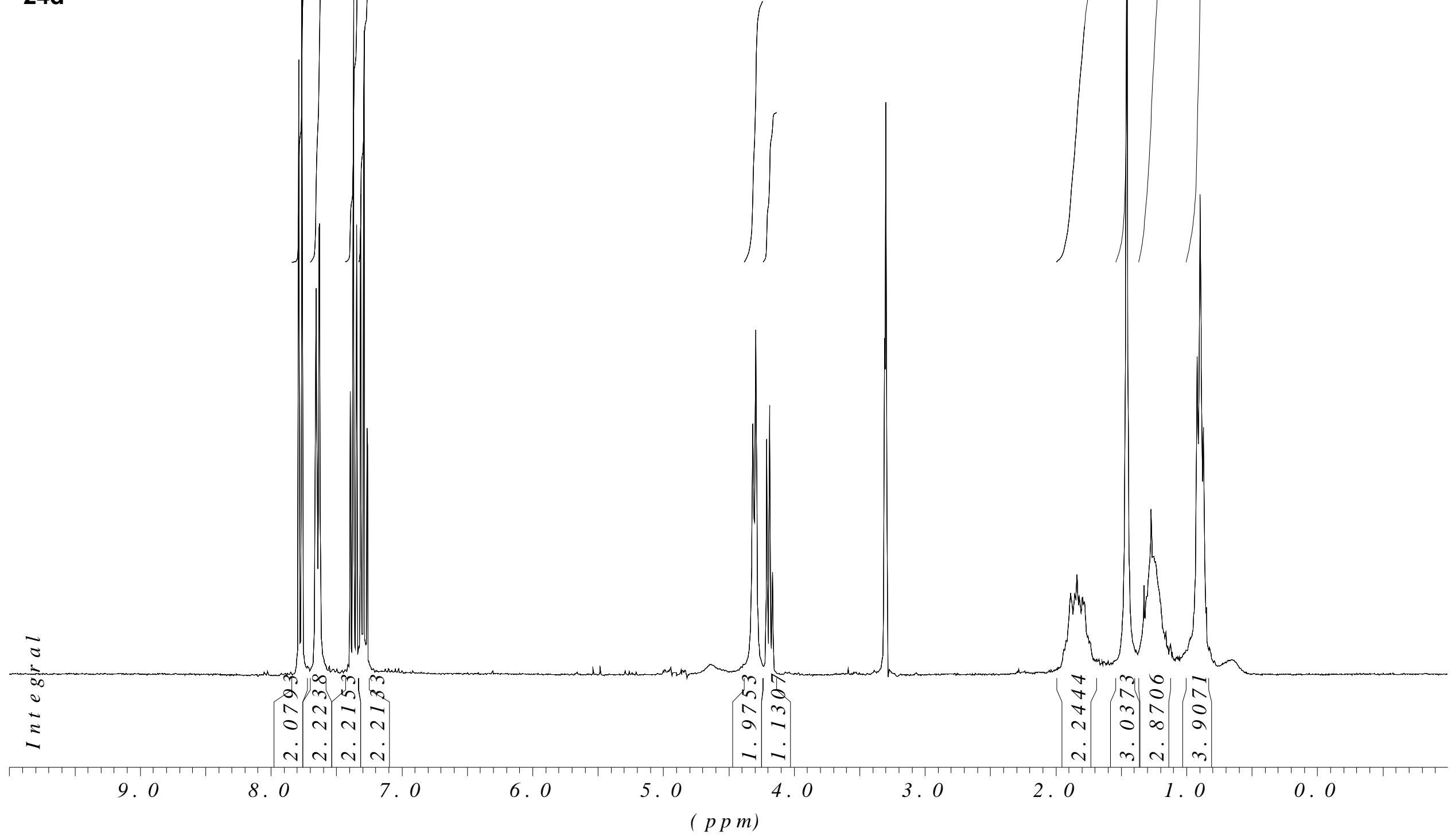

m $\infty m$

a $m$ in

$\begin{array}{lll}N & v & v\end{array}$

m $N$

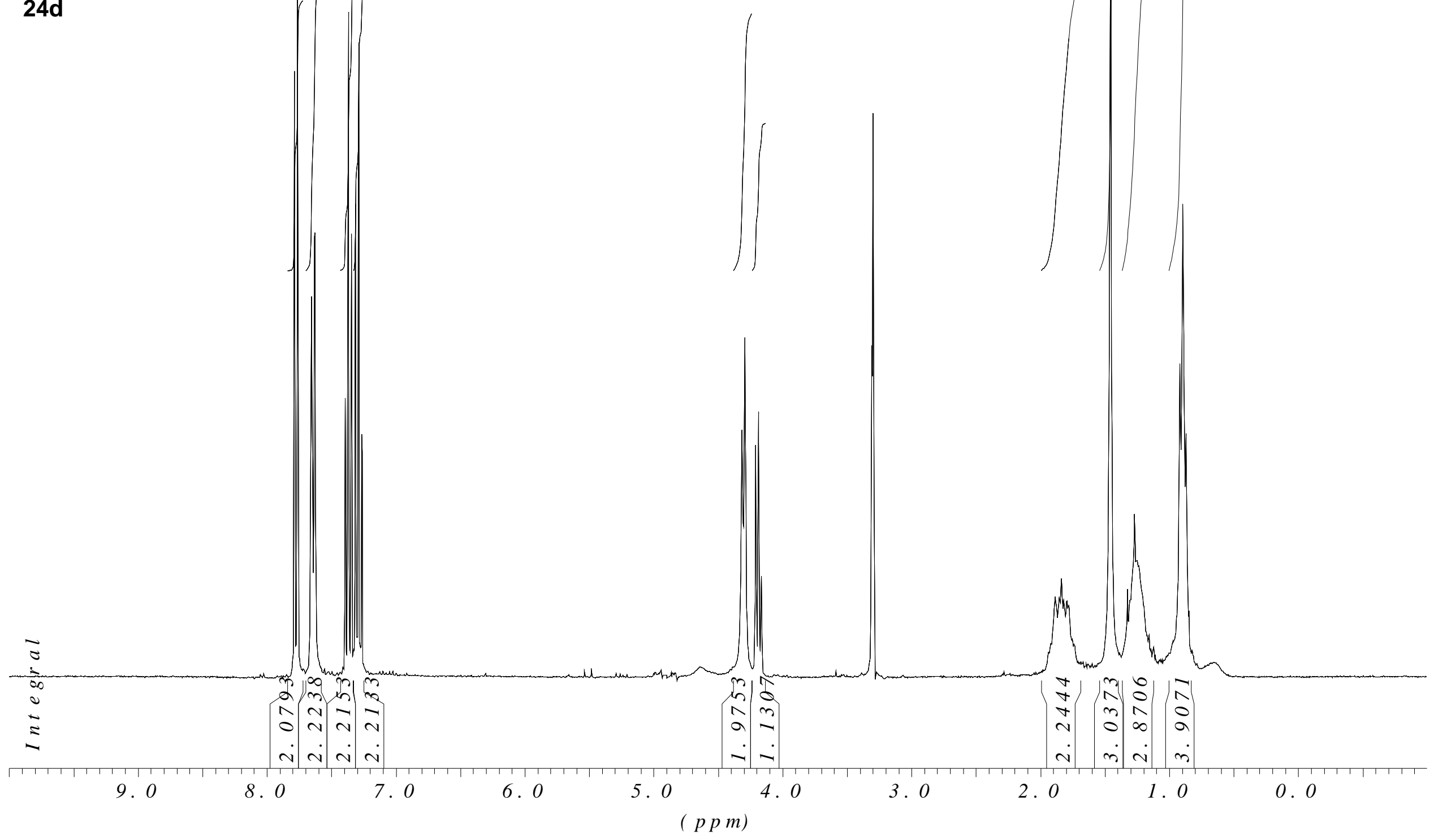

S- 87 


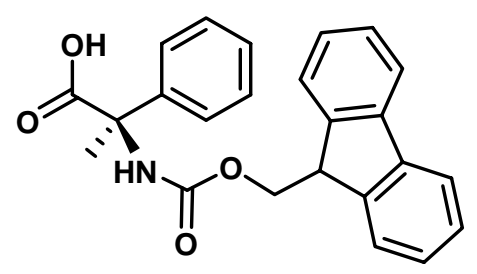

$15 e$

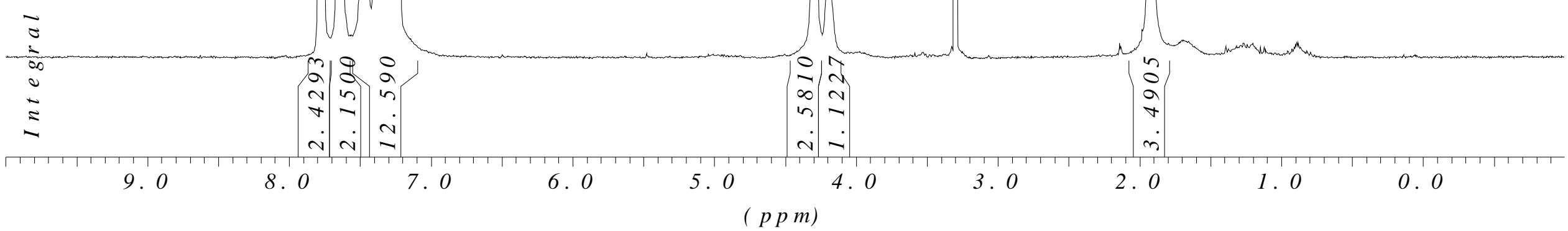




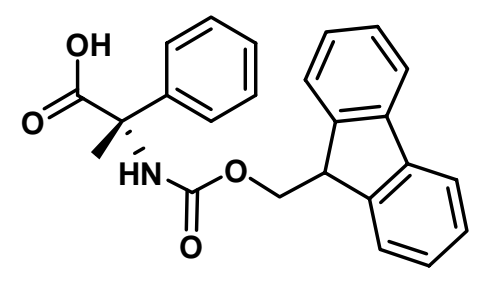

S- 89

\section{$24 e$}
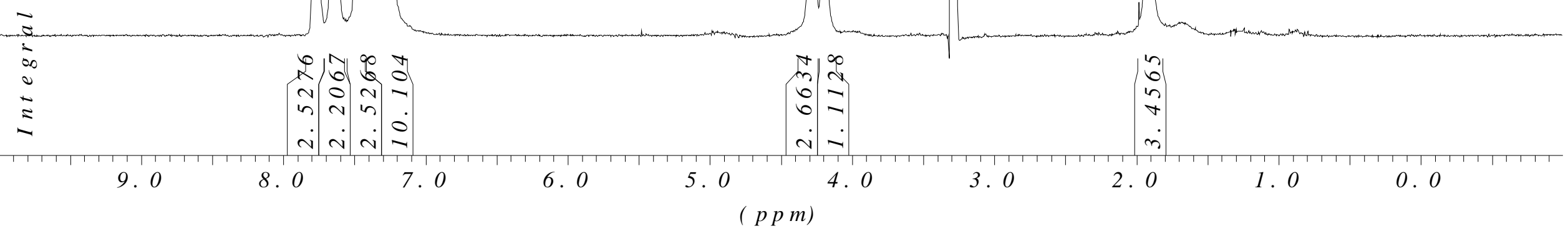


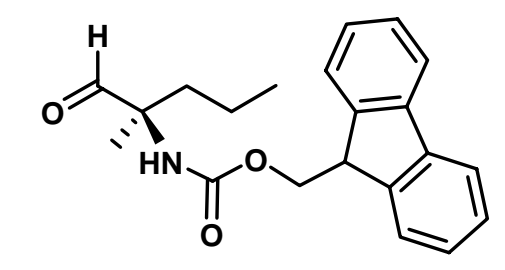

26d

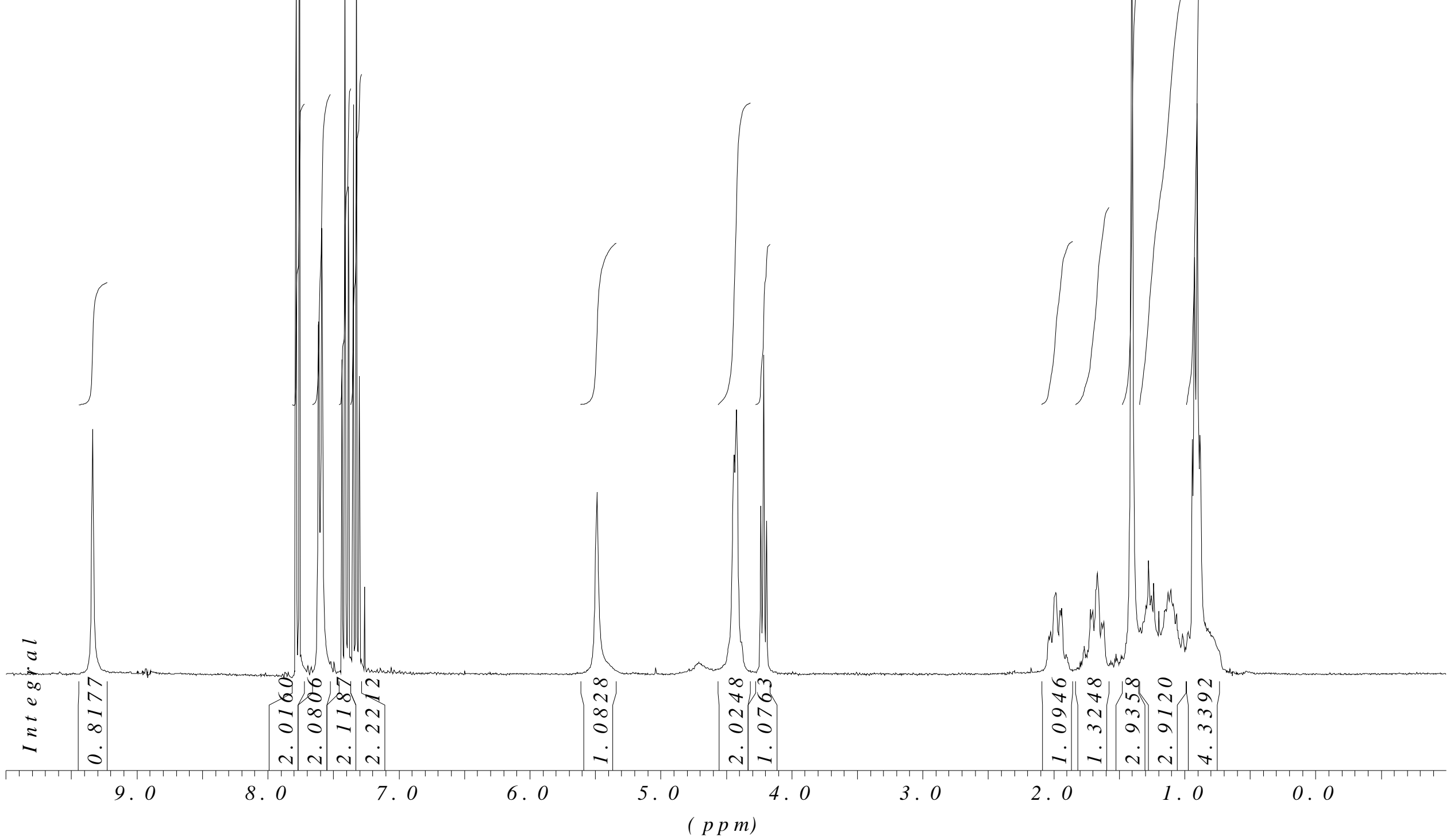




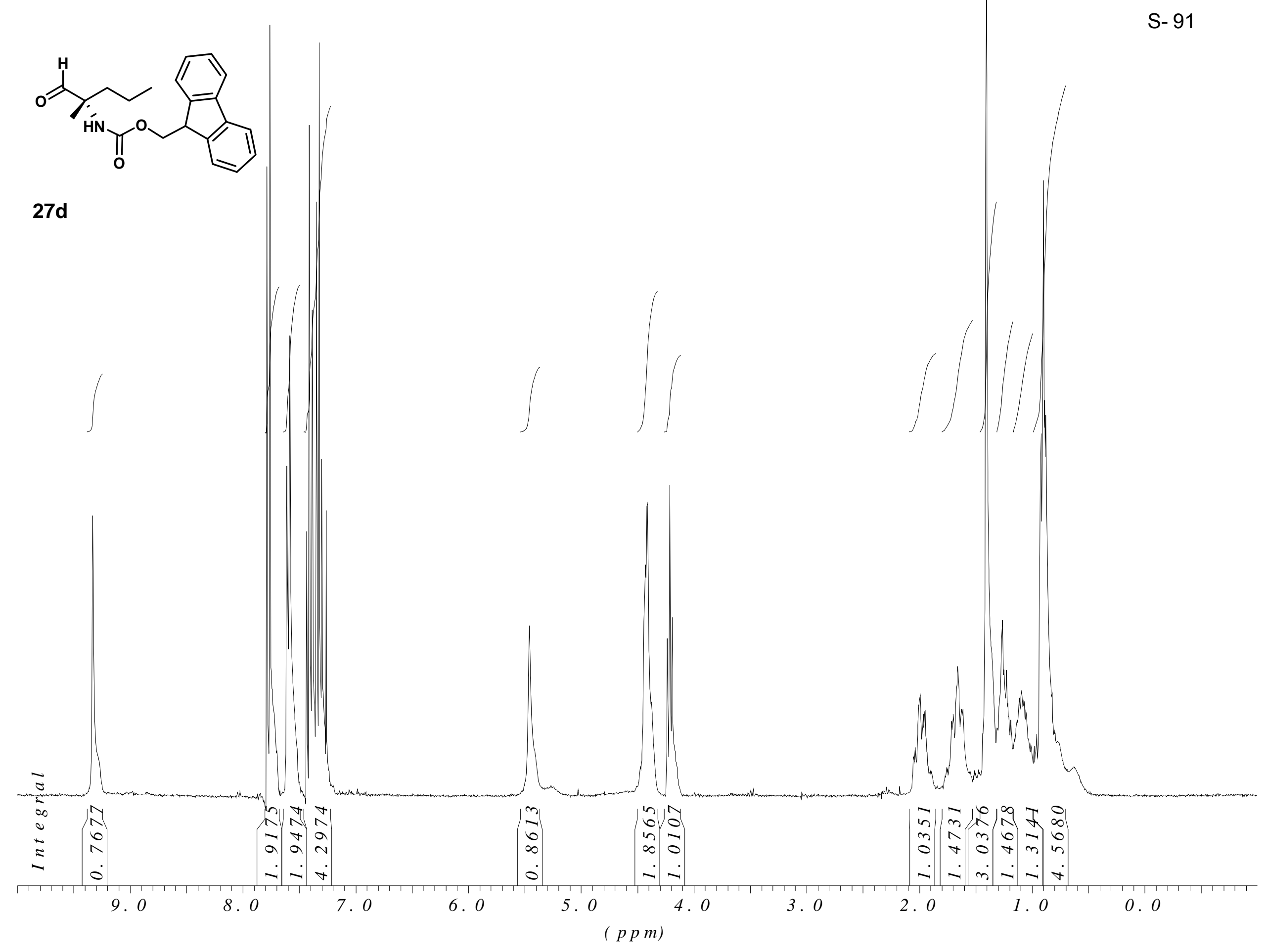



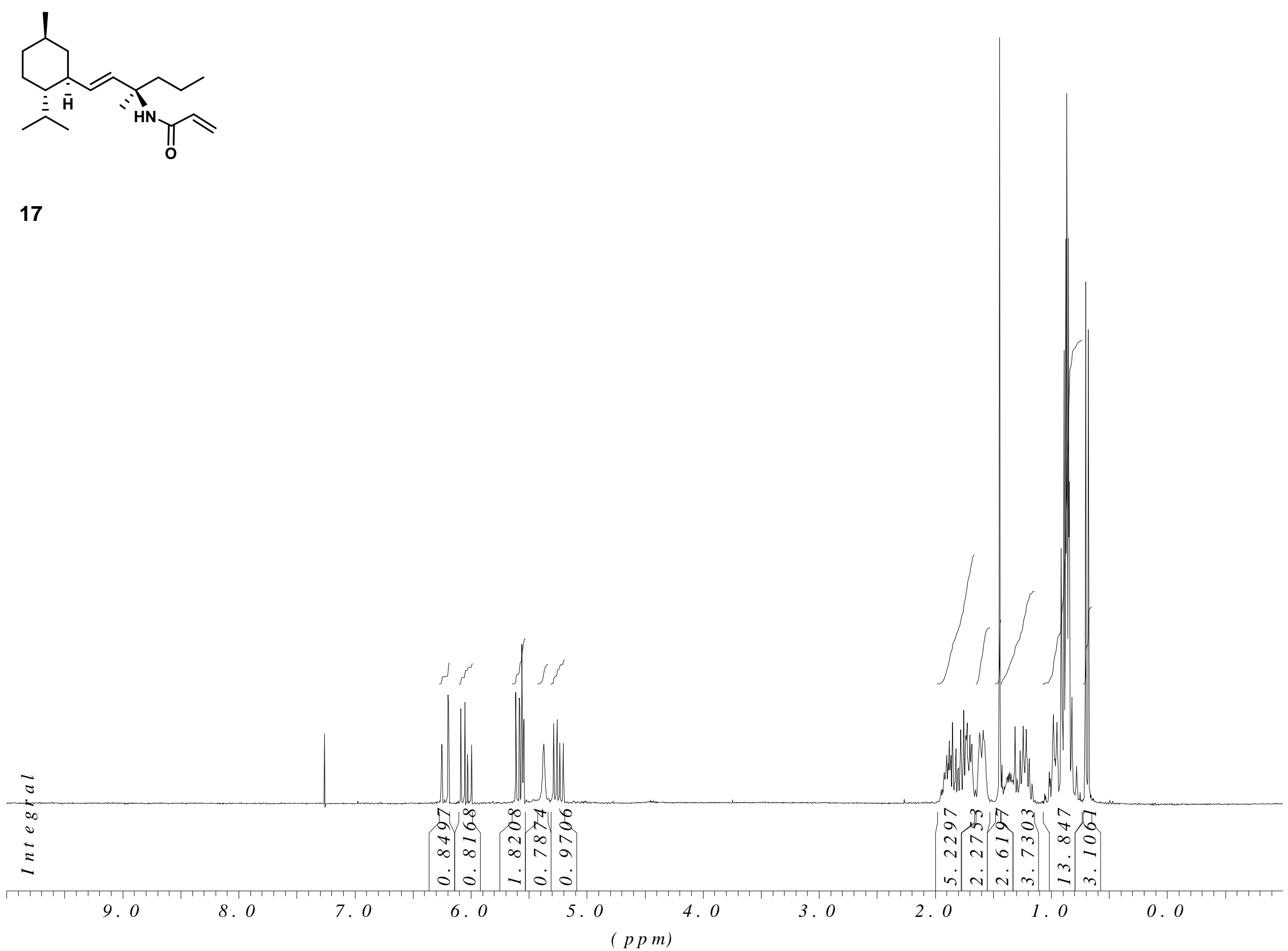


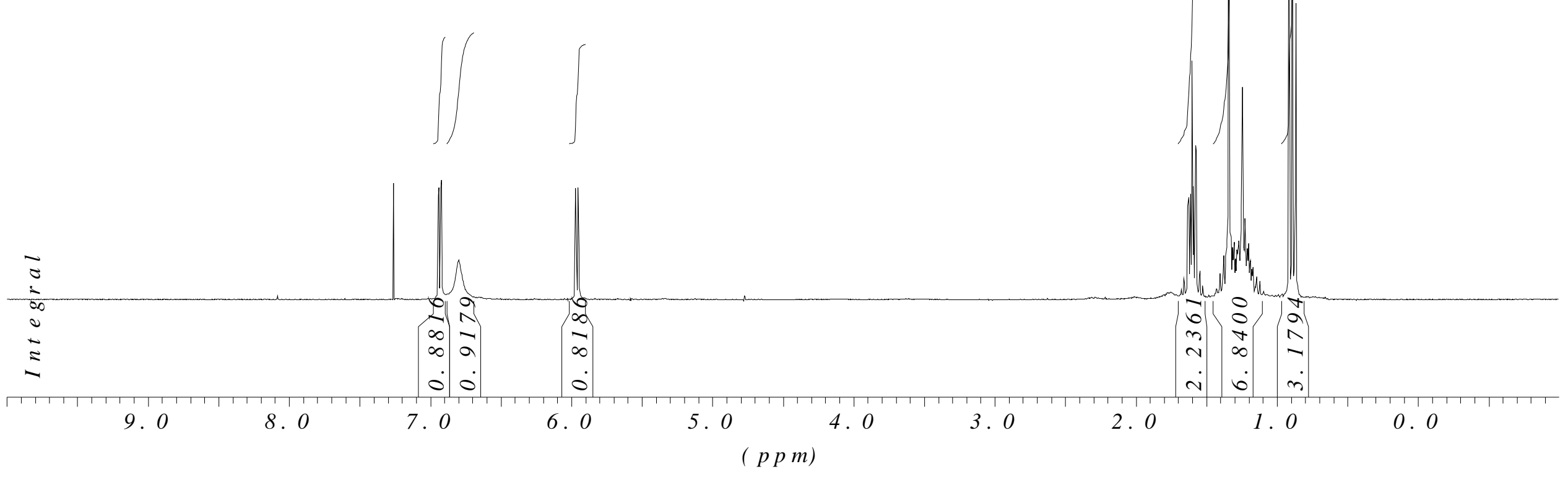

